\title{
O MÉTODO DOS ELEMENTOS DE CONTORNO APLICADO À ANÁLISE NÃO-LINEAR DE PLACAS
}

\section{GABRIELA REZENDE FERNANDES}

Dissertação apresentada à Escola de Engenharia de São Carlos, da Universidade de São Paulo, como parte dos requisitos para obtenção do Título de Mestre em Engenharia de Estruturas.

ORIENTADOR: Prof. Tit. Wilson Sergio Venturini

\section{São Carlos}


Aos meus pais, com amor. 


\section{AGRADECIMENTOS}

Desejo expressar meus agradecimentos a todos que, de alguma forma, ajudaram na realização deste trabalho.

Agradeço especialmente ao Professor Doutor Wilson Sergio Venturini pela amizade, a excelente orientação e pelo estímulo em todos os estágios deste trabalho.

Aos professores do Departamento de Engenharia Civil da UFU, em especial ao Prof. Dr. Arquimedes Diógenes Ciloni, pela orientação durante a graduação e pela amizade.

Aos professores e funcionários do Departamento de Estruturas da Escola de Engenharia de São Carlos, especialmente ao Prof. Dr. Sérgio Persival Baroncini Proença, ao Prof. Dr. João Batista de Paiva, a Rosi Aparecida Jordão Rodrigues e a Maria Nadir Minatel.

A CAPES, que através da bolsa de estudo possibilitou o desenvolvimento desta dissertação.

De modo especial, quero agradecer a Eduardo Chaves, que muito me ajudou no decorrer do desenvolvimento do trabalho, a Marcelo Cerucci pelo carinho e amizade e aos meus pais, que através do seu amor, estímulo e apoio, sempre me ajudaram nos momentos difíceis. 


\section{SUMÁRIO}

LISTA DE FIGURAS ........................................................................................................ii

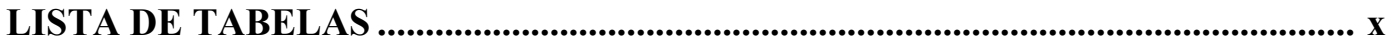

LISTA DE SÍMBOLOS .........................................................................................

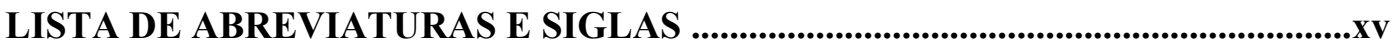

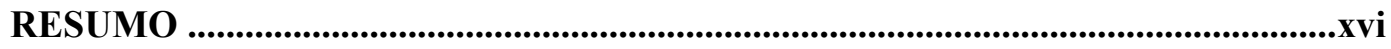

ABSTRACT .....................................................................................................................................xvii

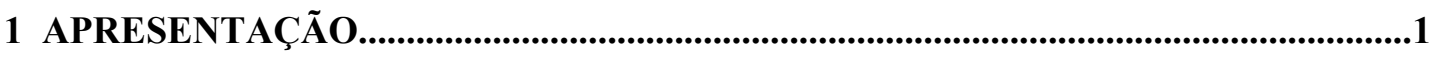

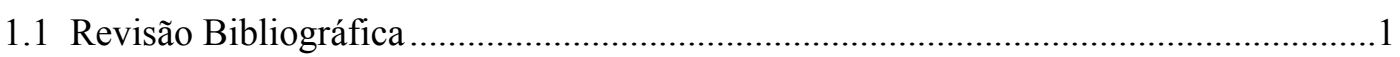

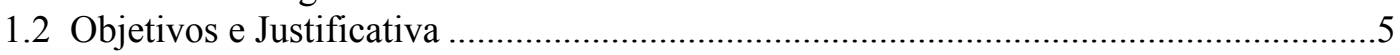

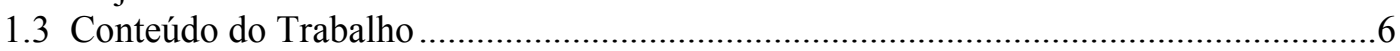

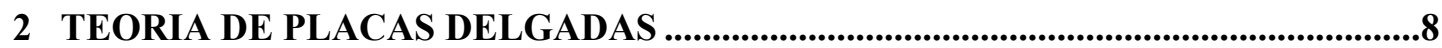

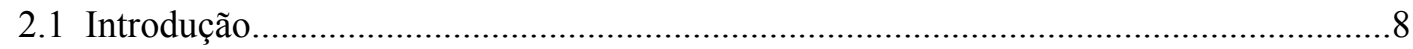

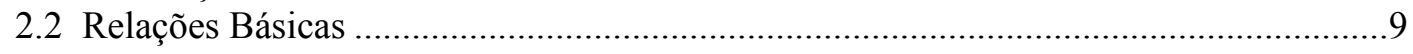

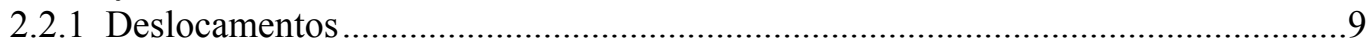

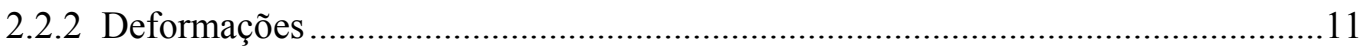

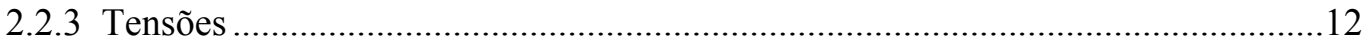

2.2.4 Esforços e Relações de Equilíbrio ........................................................................12

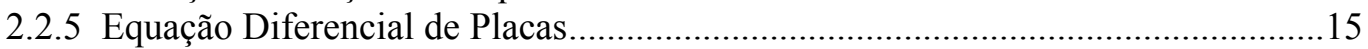

2.2 6 Esforços Segundo um Sistema de Coordenadas Genérico $(\mathrm{n}, \mathrm{s})$............................16

2.2.7 Força Cortante Equivalente e Reação de Canto........................................................17

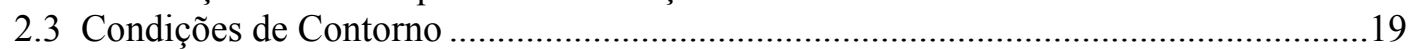

2.4 Equação Diferencial e Esforços em Coordenadas Polares ...............................................20

3 EQUAÇÕES INTEGRAIS PARA FLEXÃO DE PLACAS SUJEITAS À

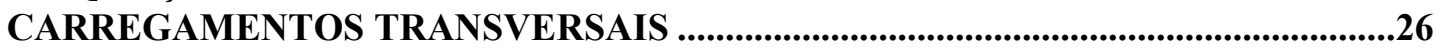

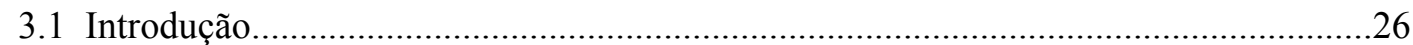

3.2 Equação Integral para um Ponto do Domínio da Placa .................................................26

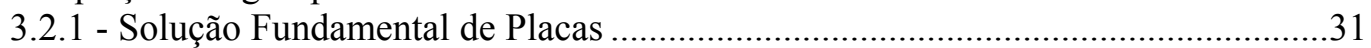

3.3 Equação Integral para um Ponto do Contorno da Placa ..................................................37

3.4 Transformação das Integrais de Domínio do Carregamento em Integrais de Contorno40

\section{MÉTODO DOS ELEMENTOS DE CONTORNO APLICADO A PROBLEMAS DE} PLACAS SUJEITAS A CARGAS TRANSVERSAIS ................................................43

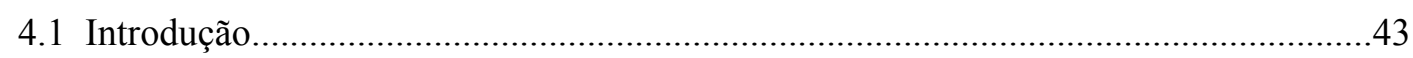

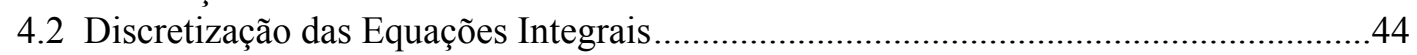

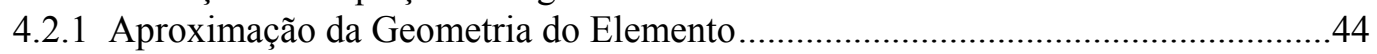

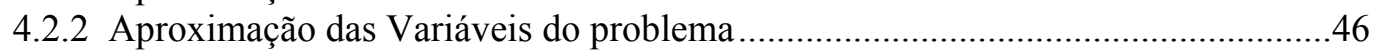

4.2.3 Transformações das Equações Integrais em Equações Algébricas........................50

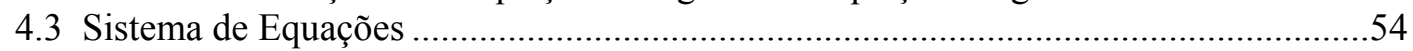

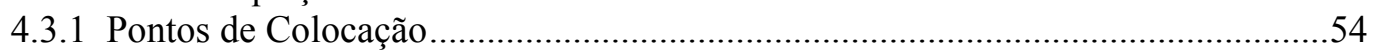

4.3.2 Equação de Deslocamento para um Ponto Externo .................................................56

4.3.3 Obtenção das Incógnitas do Contorno e dos Cantos ............................................56

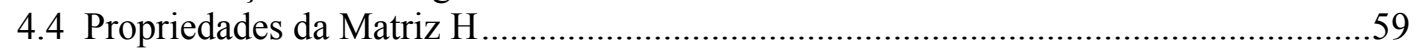

4.5 Deslocamentos e Esforços para Pontos Internos .........................................................61 


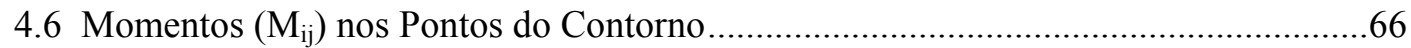

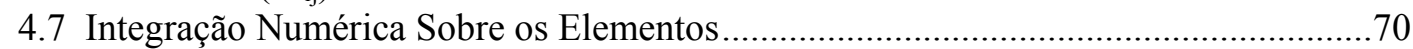

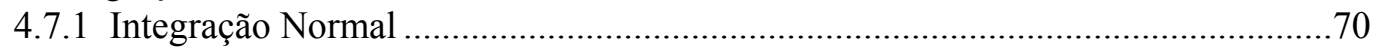

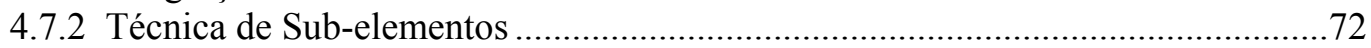

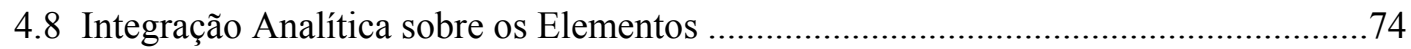

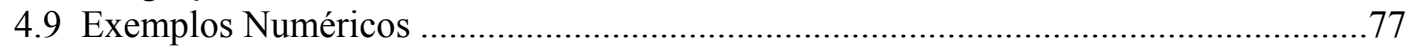

4.9.1 Placa quadrada com dois lados opostos apoiados e os demais engastados,

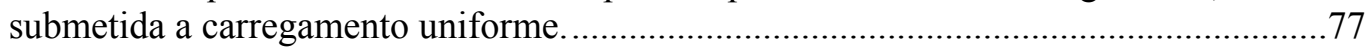

4.9.2 Placa quadrada apoiada nos quatro cantos e submetida a carga uniformemente

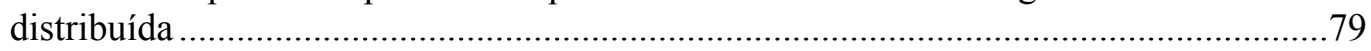

4.9.3 Placa quadrada engastada nos quatro lados, com carregamento linearmente

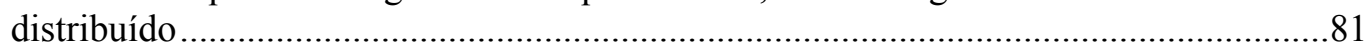

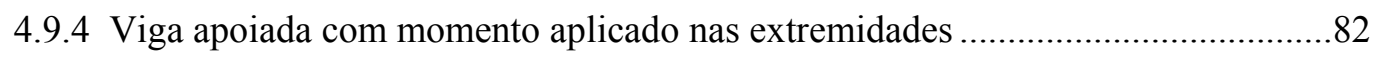

\section{PROBLEMA DE PLACAS COM CAMPO DE MOMENTOS INICIAIS....................84}

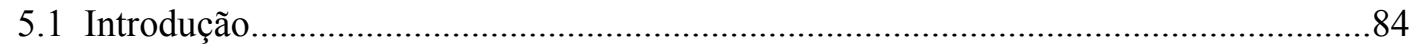

5.2 Equações Integrais com Campo de Momentos Iniciais .................................................84

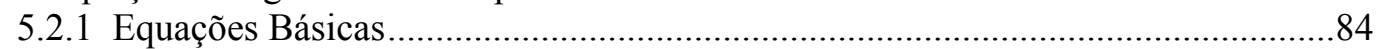

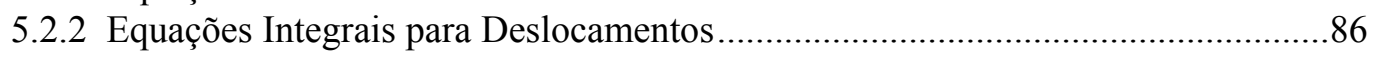

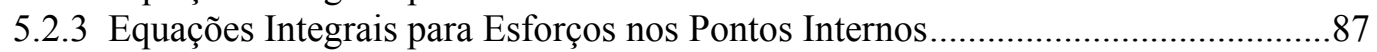

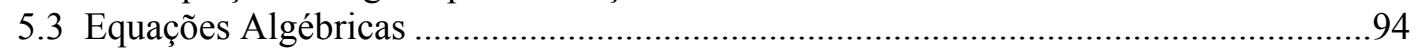

5.3.1 Discretização do Domínio da Placa .....................................................................95

5.3.2 Aproximação da geometria da célula e do Campo de Momentos Iniciais .............95

5.3.3 Integração sobre as Células ...................................................................................97

5.4 Integração Numérica Sobre as Células .........................................................................99

5.4.1 Integração sobre as células referente à equação do deslocamento $\mathrm{w}$ de um ponto do contorno ou do domínio........................................................................................99

5.4.2 Integração sobre as Células Referente à Equação das Curvaturas de um Ponto

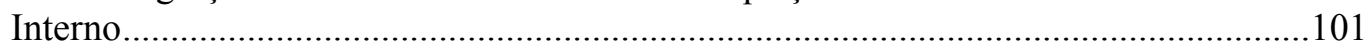

5.4.3 Integração sobre as Células Referente à Equação das Derivadas das Curvaturas de

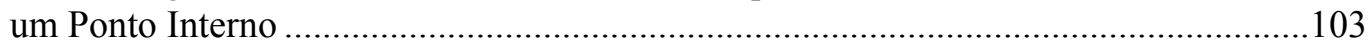

5.5 Cálculo dos Deslocamento e Esforços no Contorno e no Domínio ..............................105

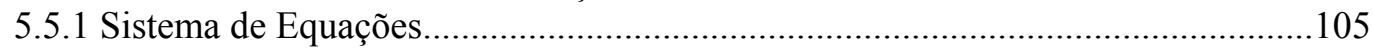

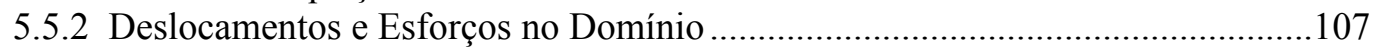

5.5.3 Momentos $\left(\mathrm{M}_{\mathrm{ij}}\right)$ nos Pontos do Contorno …….................................................108

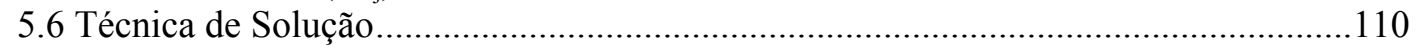

5.6.1 Cálculo das Incógnitas do Contorno e Deslocamentos w do Domínio..................110

5.6.2 Cálculo dos Esforços nos Pontos Internos e no Contorno ....................................112

6 MODELOS CONSTITUTIVOS UTILIZADOS .......................................................115

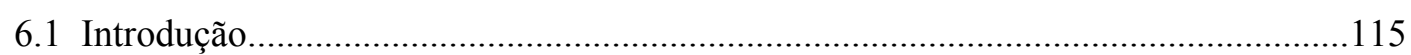

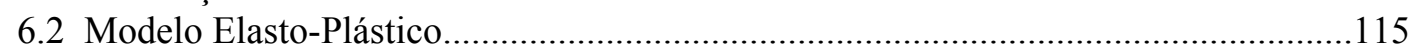

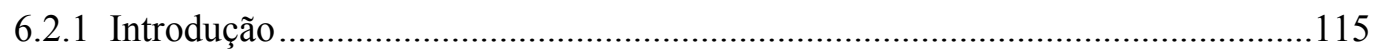

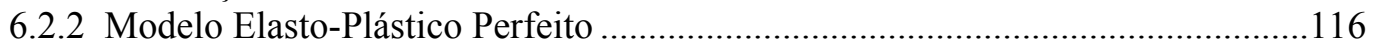

6.2.3 Modelo Elasto-Plástico com Encruamento Isótropo Positivo .............................120

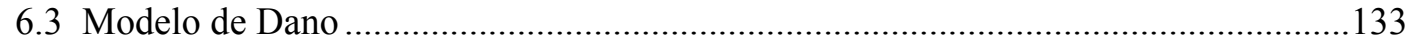

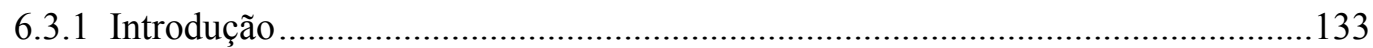

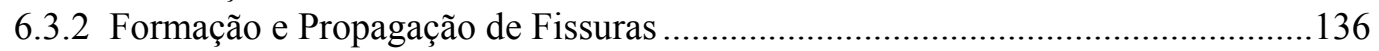

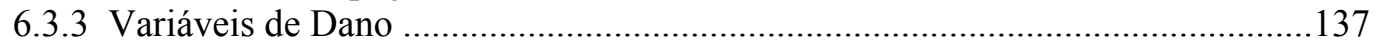

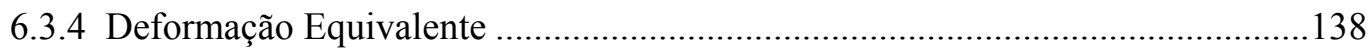

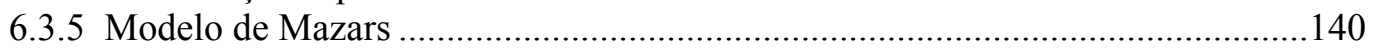




\section{SOLUĈ̃O NÃO-LINEAR DE UMA PLACA SUJEITA A CARGAS}

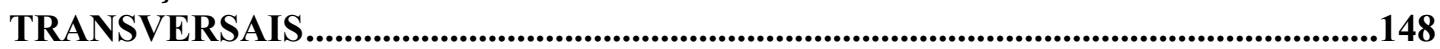

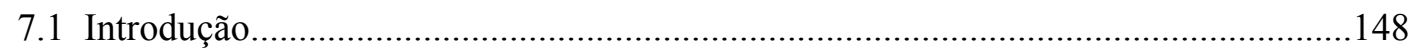

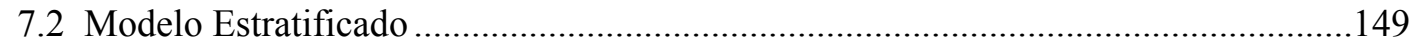

7.2.1 Cálculo do Momento Interno Resultante em uma Seção da Placa .......................149

7.2.2 Processo para Zerar a Normal Resultante numa Seção da Placa ...........................151

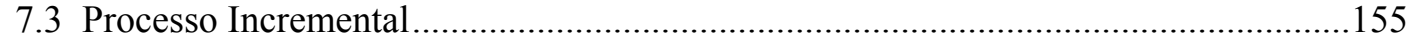

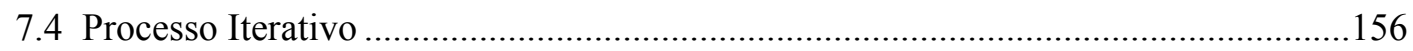

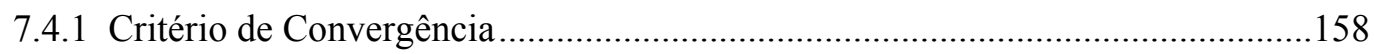

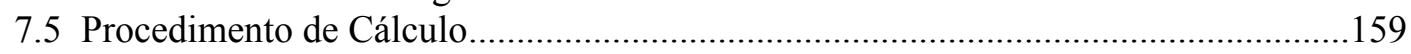

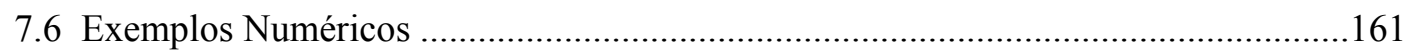

7.6.1 Viga apoiada com momento aplicado nas extremidades ....................................161

7.6.2 Placa apoiada com carga concentrada no centro ..................................................165

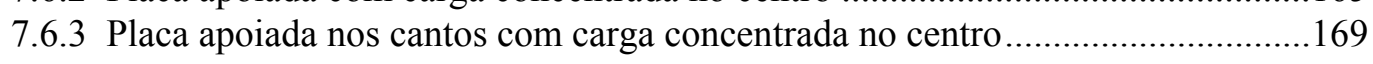

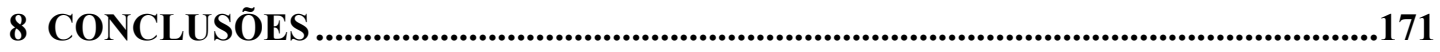

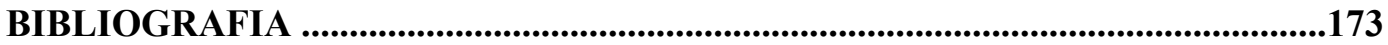




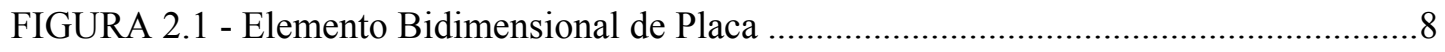

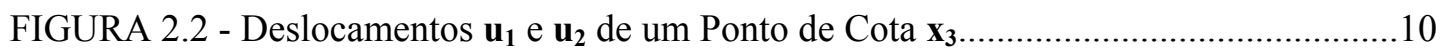

FIGURA 2.3 - Tensões em um Elemento de Placa.................................................................13

FIGURA 2.4 - Esforços em um Elemento de Placa .............................................................13

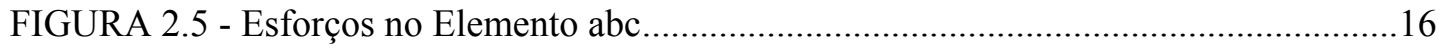

FIGURA 2.6 - Momentos Volventes no Contorno da Placa......................................................18

FIGURA 2.7 - Momentos Volventes em um Canto i da Placa ................................................19

FIGURA 2.8 - Placa com Condições de Contorno. ...............................................................20

FIGURA 2.9 - Sistema de Coordenadas Polares .................................................................20

FIGURA 2.10 - - Vetores n e s no Ponto P do Contorno da Placa ..........................................23

FIGURA 3.1 - Placa de Dimensões Finitas, Contida em uma Placa Infinita ............ 27

FIGURA 3.2 - Pontos de Carregamento q e de Deslocamento p e Sistemas de

Coordenadas (m, u) e (n, s) no Domínio de uma Placa Infinita................................ 28

FIGURA 3.3 - Forças Atuantes no Círculo de Raio r e Centro q.............................. 32

FIGURA 3.4 - Contorno Circular Acrescido a um ponto $\mathbf{Q}$ de um Canto da Placa .. 37

FIGURA 3.5 - Região Carregada $\Omega_{\mathrm{g}}$ de uma Placa de Domínio $\Omega$........................... 41

FIGURA 4.1 - Discretização do Contorno da Placa .................................................................44

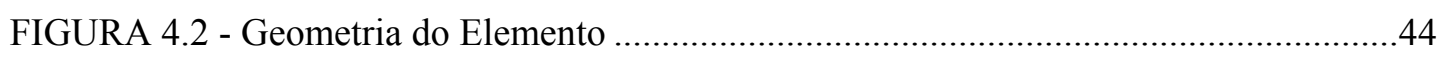

FIGURA 4.3 - Funções de Forma em Aproximação Quadrática das Variáveis......................46

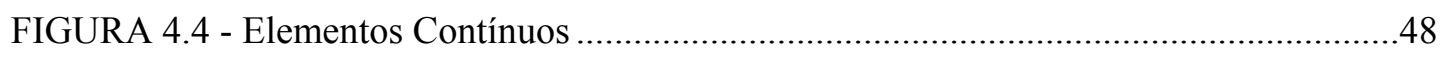

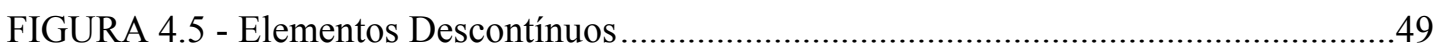

FIGURA 4.6 - Elemento de Contorno Descontínuo ……………………….............................49

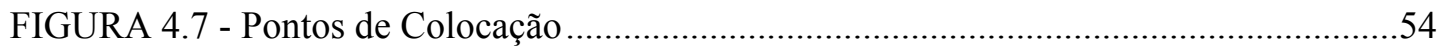

FIGURA 4.8 - Dois Pontos de Colocação no Contorno ……...................................................55

FIGURA 4.9 - Movimento de Corpo Rígido - Translação ………………………………….....60

FIGURA 4.10 - Movimento de Corpo Rígido - Rotação..........................................................60

FIGURA 4.11 - Sistema Local de Coordenadas de um Elemento do Contorno .......................66

FIGURA 4.12 - Divisão de um Elemento em Sub-elementos ...................................................73

FIGURA 4.12 - Elemento de Contorno Descontínuo ………………………..........................76

FIGURA 4.13 - Placa Quadrada Apoiada em Dois Lados Opostos e Engastada nos outros

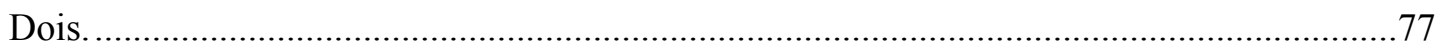

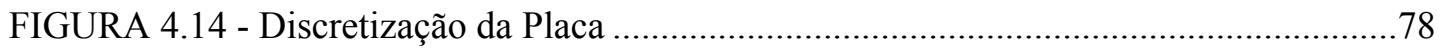

FIGURA 4.15 - Placa Quadrada Apoiada nos Quatro Cantos ……………………................80 
FIGURA 4.16 - Placa Engastada

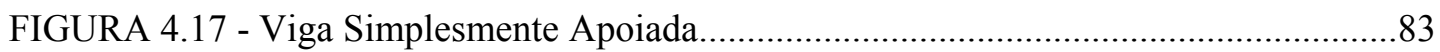

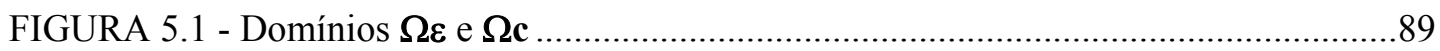

FIGURA 5.2 - Divisão do Domínio da Placa em Células Triangulares e a Variação dos

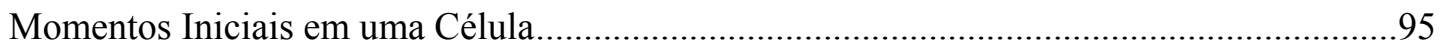

FIGURA 5.3 - Célula Triangular com Sistema de Coordenadas Homogêneas .......................95

FIGURA 5.4 - Sistema de Coordenadas Cilíndricas ................................................................98

FIGURA 5.5 - Sistema de Coordenadas Cilíndricas, com Origem no Vértice da Célula.....103

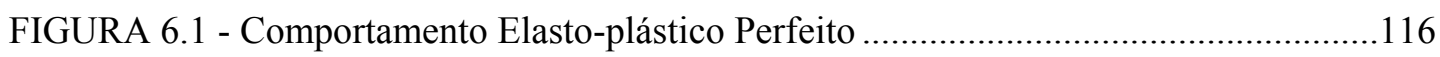

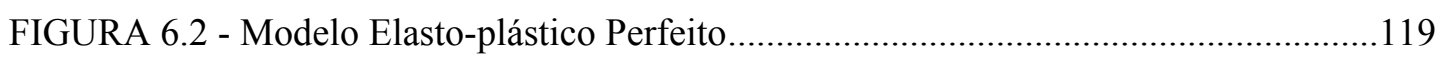

FIGURA 6.3 - Caso Uniaxial de Encruamento Linear Isótropo ..........................................121

FIGURA 6.4 - Modelo Elasto-Plástico com Encruamento ......................................................123

FIGURA 6.5 - Correção da Tensão, num Modelo Elasto-plástico Uniaxial com Encruamento

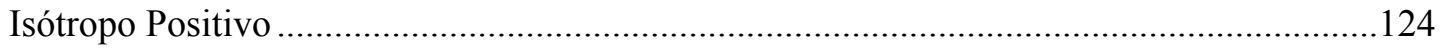

FIGURA 6.6 - Representação Geométrica do Critério de Von Mises no caso biaxial .........128

FIGURA 6.7 - Representação Geométrica do Critério de Von Mises no caso biaxial, com

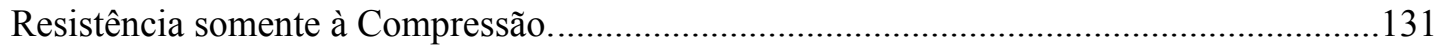

FIGURA 6.8 - Correção das Tensões para o Modelo Uniaxial de Encruamento Isótropo

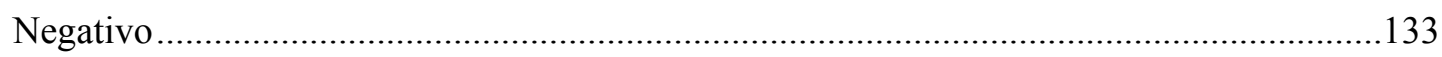

FIGURA 6.9 - Modelo de Dano Uniaxial no Concreto ............................................................134

FIGURA 6.10 - Tipos de Abertura de Fissura .................................................................137

FIGURA 6.11 - Elemento de Volume com Dano …...............................................................137

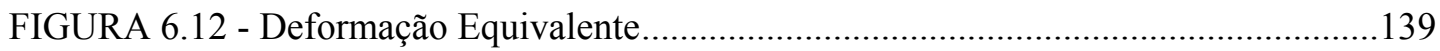

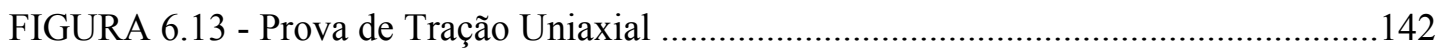

FIGURA 6.14 - Superfície de Ruptura em Deformação ........................................................143

FIGURA 6.15 - Superfície de Ruptura em Tensão ..............................................................144

FIGURA 6.16 - Microfissuração em Tração e Compressão Uniaxiais....................................144

FIGURA 6.17 - Modelo de MAZARS em Tração e Compressão …………………..............147

FIGURA 7.1 - Modelo Estratificado para o Concreto Armado ..............................................149

FIGURA 7.2 - Estimativa da Linha Neutra ....................................................................152

FIGURA 7.3 - Distribuição das Deformações em uma Seção da Placa...................................153

FIGURA 7.4 - Distribuição de Deformação Resultante em uma Seção .................................154

FIGURA 7.5 - Distribuição de Tensão Resultante em uma Seção .........................................155

FIGURA 7.6 - Método de Newton Raphson Padrão ...........................................................156

FIGURA 7.7 - Método de Newton Raphson Modificado .......................................................158 


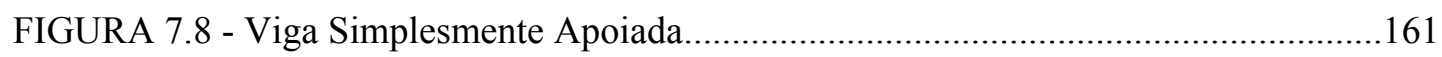

FIGURA 7.9 - Discretização da viga ...............................................................................162

FIGURA 7.10 - Deslocamentos Transversais nos Pontos do Domínio .................................162

FIGURA 7.11 - Curva Carga-deslocamento do Ponto Central...............................................163

FIGURA 7.12 - Influência do Número de Pontos de Gauss (NG) na Curva Carga-

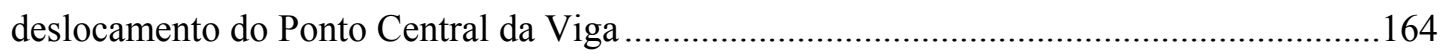

FIGURA 7.13 - Placa Apoiada com Carga Concentrada......................................................165

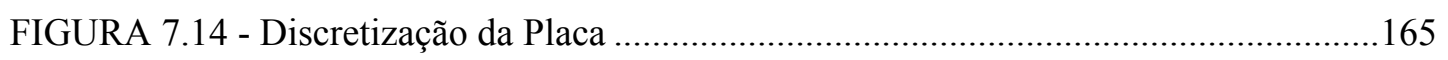

FIGURA 7.15 - Curva Carga-Deslocamento no Ponto Central ............................................166

FIGURA 7.16 - Curva Momento-Curvatura na Direção $x_{1}$ no ponto Central .......................167

FIGURA 7.17 -Curva Carga-Deslocamento no Ponto Central, Considerando o Modelo de

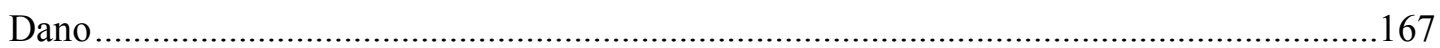

FIGURA 7.18 -Influência do Número de Pontos de Gauss na Curva Carga-Deslocamento do

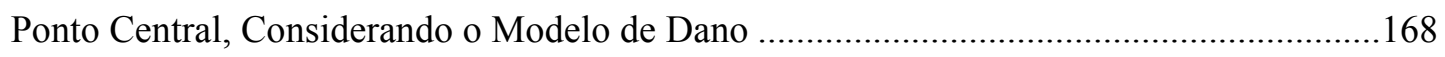

FIGURA 7.19 - Placa Apoiada nos Cantos com Carga Concentrada ...................................169

FIGURA 7.20 -Curvas Carga-Deslocamento para o Ponto 1, Considerando Duas Malhas

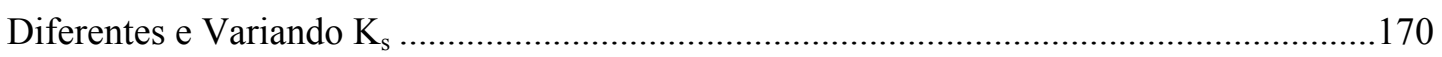

\section{LISTA DE TABELAS}

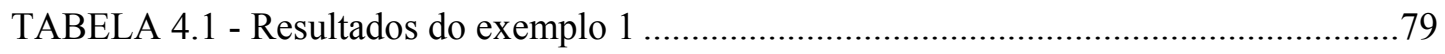

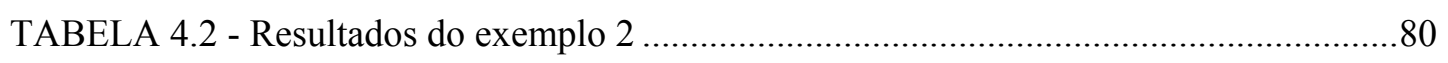

TABELA 4.3 - Placa engastada com carga linearmente distribuída .......................................82

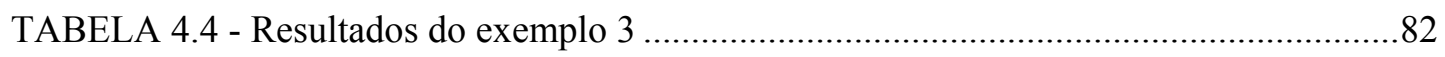

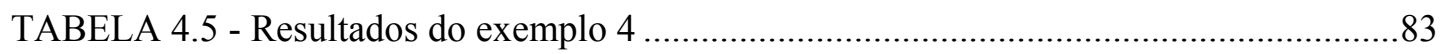


TABELA 7.1 - Resultados obtidos para $\beta=0,9$ 163

TABELA 7.2 - Comparação dos resultados obtidos com as duas malhas 164

TABELA 7.3 - Influência do número de pontos de Gauss no deslocamento e na carga limite168

\section{LISTA DE SÍMBOLOS}

$\begin{array}{ll}\nabla^{2} & : \text { operador diferencial escalar, } \nabla^{2}=\frac{\partial^{2}}{\partial \mathbf{x}_{1}^{2}}+\frac{\partial^{2}}{\partial \mathbf{x}_{2}^{2}}+\frac{\partial^{2}}{\partial \mathbf{x}_{3}^{2}} \\ \Gamma & : \text { coordenada que percorre o contorno; } \\ \Gamma_{1}, \Gamma_{2} & : \text { coordenadas dos limites do contorno no qual se realiza a integração; } \\ \Gamma_{\infty} & : \text { contorno infinito; }\end{array}$




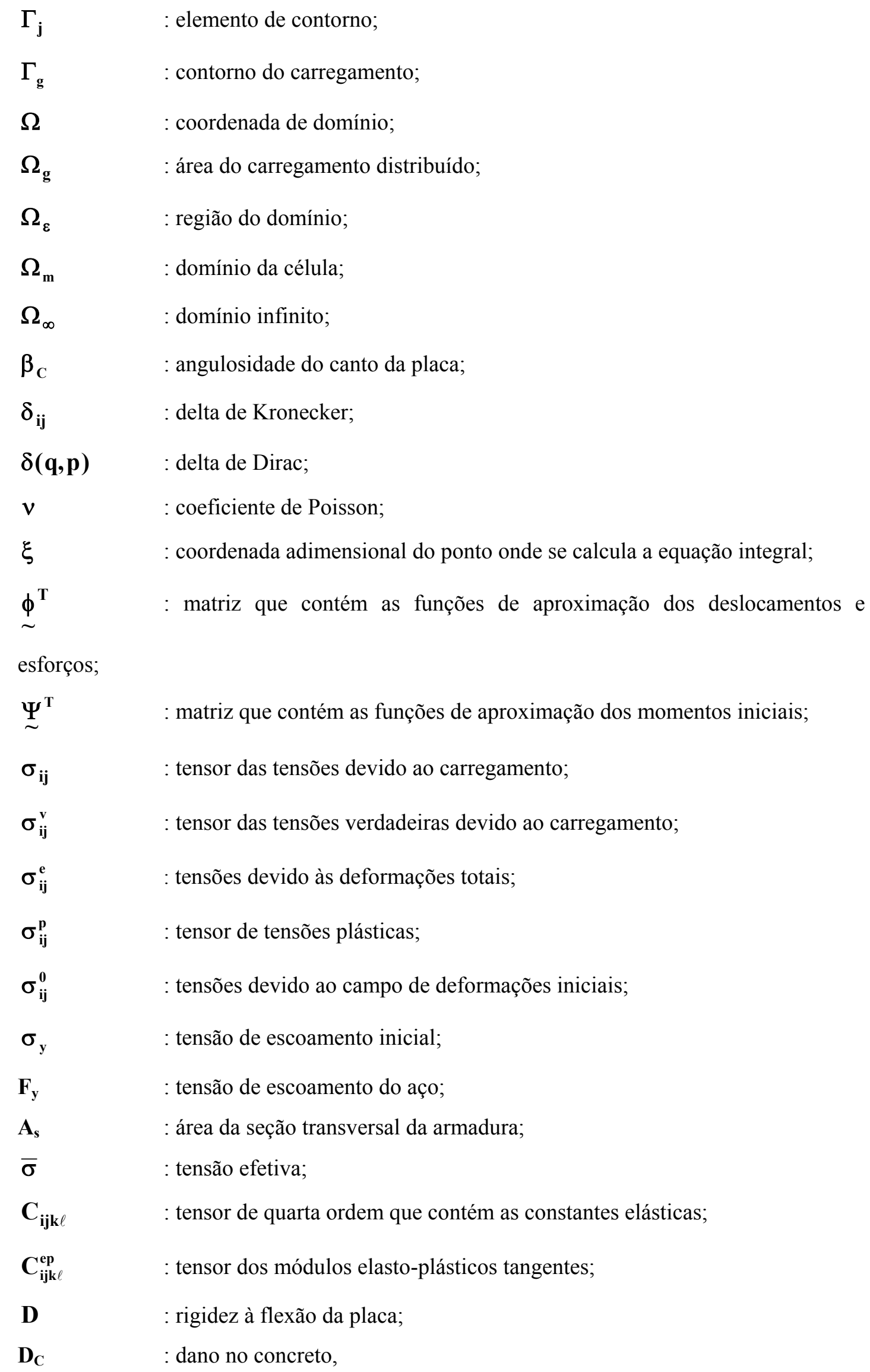




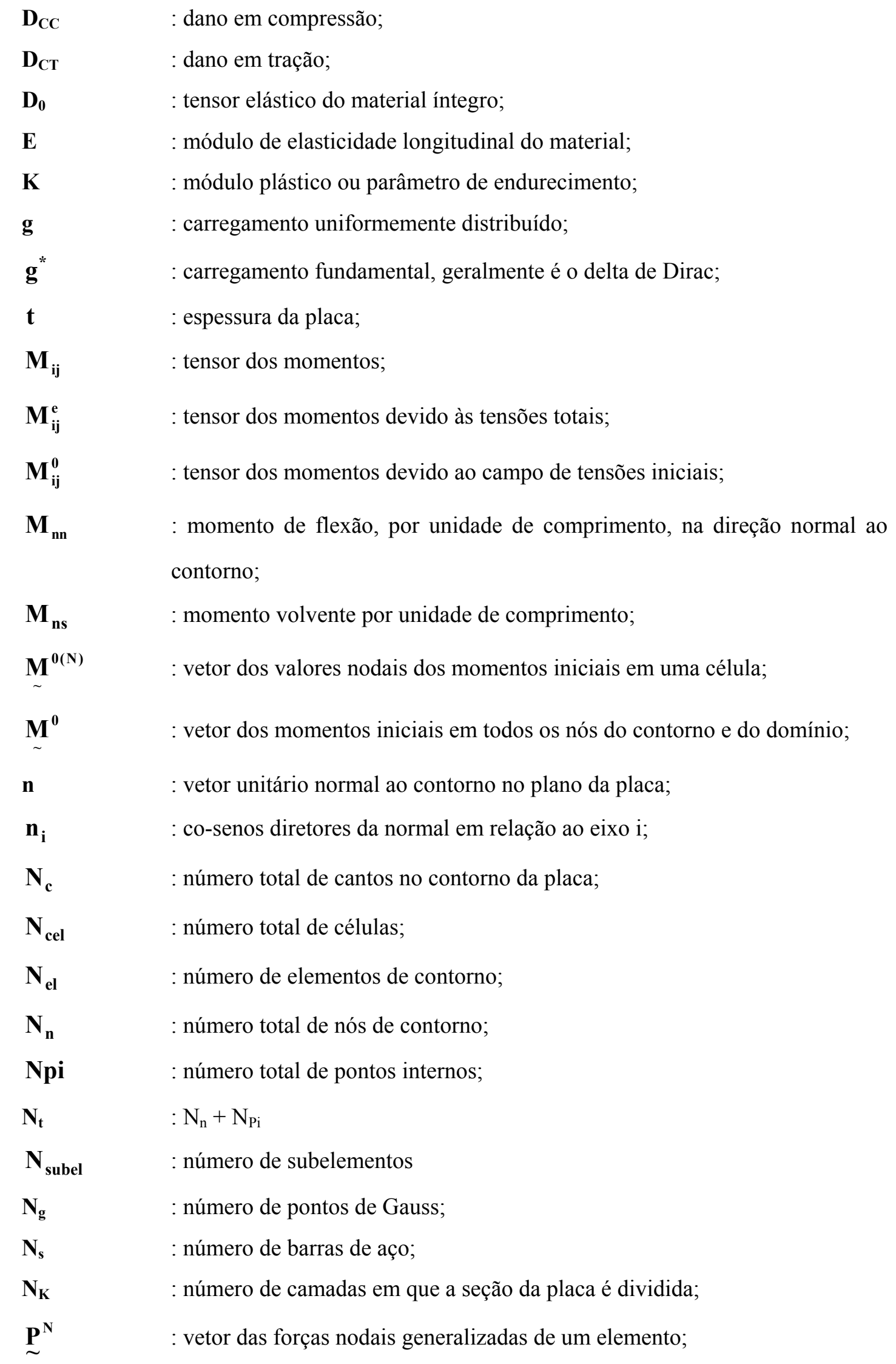




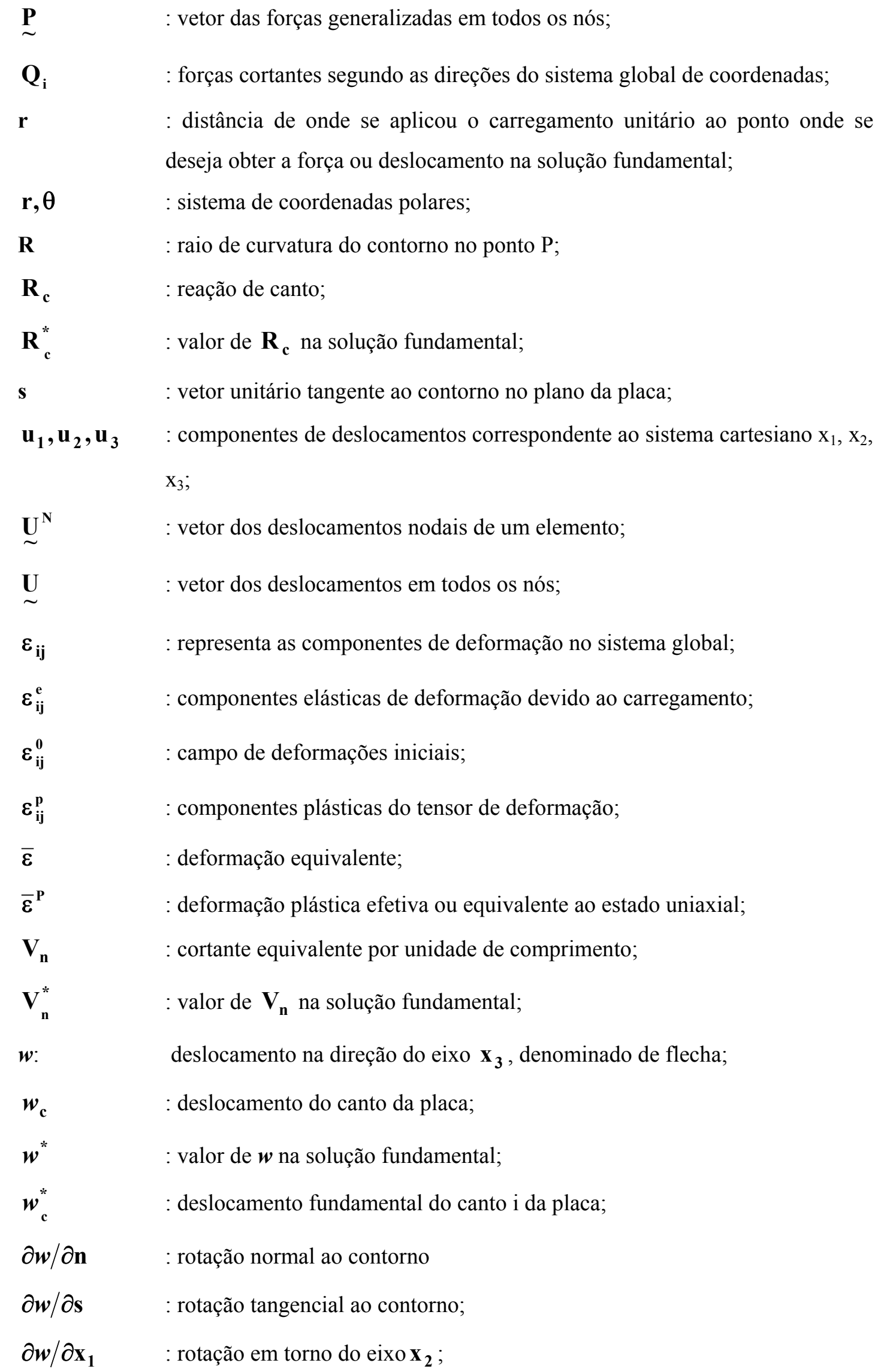


$\partial w / \partial \mathbf{x}_{2} \quad$ : rotação em torno do eixo $\mathbf{x}_{1}$;

$\partial \boldsymbol{w}^{*} / \partial \mathbf{n} \quad:$ valor de $\partial \boldsymbol{w} / \partial \mathbf{n}$ na solução fundamental;

$w_{9_{\mathrm{ij}}} \quad$ : curvaturas;

$\boldsymbol{w}, \quad$ : valor de ${ }^{*},_{\mathrm{ij}}$ na solução fundamental;

$\mathbf{x}_{1}, \mathbf{x}_{2}, \mathbf{x}_{3} \quad$ : sistema de coordenadas cartesianas;

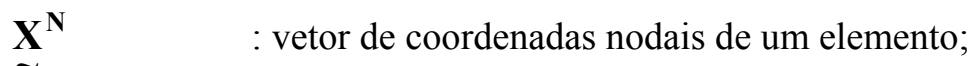




\title{
LISTA DE ABREVIATURAS E SIGLAS
}

\author{
MEF - Método dos Elementos Finitos; \\ MEC - Método dos Elementos de Contorno; \\ BEM - Boundary Element Method
}




\section{RESUMO}

FERNANDES, G.R. (1998). O método dos elementos de contorno aplicado à análise não linear de placas. São Carlos. 178p. Dissertação (Mestrado) - Escola de Engenharia de São Carlos, Universidade de São Paulo.

Neste trabalho, desenvolve-se uma formulação linear de placas através do Método dos Elementos de Contorno, baseada na teoria clássica de Kirchhoff, onde a integração numérica, sobre os elementos do contorno, é feita considerando-se a técnica de subelementos. Estende-se essa formulação à análise não-linear de placas de concreto armado, através da inclusão de um campo de momentos iniciais, onde as integrais de domínio são calculadas aproximando-se o campo de momentos iniciais em células internas. Consideramse dois modelos constitutivos para o concreto: um elasto-plástico, onde o critério utilizado é o de Von Mises, sem considerar resistência à tração, enquanto que o outro é o modelo de dano de Mazars. A distribuição das tensões é aproximada, em uma seção qualquer da placa, por pontos discretos, que seguindo um esquema gaussiano, permite a integração numérica para o cálculo dos esforços. Em cada ponto, considerado ao longo da espessura, verifica-se o modelo constitutivo adotado. Numa primeira aproximação, considera-se que a linha neutra é definida pela superfície média da placa e, numa aproximação seguinte, a posição da mesma é calculada de tal forma que a força normal resultante seja nula.

Palavras-chave: Elementos de contorno, análise não-linear, flexão de placas.

\section{ABSTRACT}


FERNANDES, G.R. (1998). O método dos elementos de contorno aplicado à análise não linear de placas. São Carlos. 178p. Dissertação (Mestrado) - Escola de Engenharia de São Carlos, Universidade de São Paulo.

In this work a linear boundary element formulation for Kirchhoff plate in bending is presented, including a precise numerical scheme based on sub-element integration to compute the boundary matrices. Then, the formulation is extended to perform non-linear analysis of reinforced concrete slabs by incorporating initial moment fields. The domain integral required to evaluate the initial moment influences are performed by using the well known cell sub-division. The non-linear behaviour of concrete slabs is included by considering two particular models: The Von Mises criterion modified to consider no strength in tension and the damage model proposed by Mazars. Those criteria are verified at points along the plate thickness, appropriately placed to allow performing numerical integration to approach moments and normal forces using Gauss point schemes. A first model was developed assuming that the plate middle surface is coincident with the plate neutral axes. Then, this model is modified to find the proper neutral axis positions by enforcing the normal force resultants to be zero.

Key Words: Boundary Elements, non-linear problems, Plate Bending 


\section{APRESENTAÇÃO}

\subsection{Revisão Bibliográfica}

Grande parte dos problemas em engenharia apresenta complexidade na geometria do sólido ou é constituído de materiais cujas leis constitutivas são bastantes complexas. Assim sendo, as soluções analíticas dos mesmos, que correspondem às soluções exatas, são praticamente impossíveis de serem obtidas, sendo então necessário a obtenção de soluções aproximadas através de métodos numéricos, onde faz-se também simplificações nas leis constitutivas dos materiais e na geometria do sólido.

O trabalho proposto trata-se da análise não-linear de placas delgadas de concreto armado, sujeitas à flexão simples, através do Método dos Elementos de Contorno, considerando-se dois modelos constitutivos para o concreto: o elasto-plástico e o de dano.

O primeiro trabalho sobre a teoria de placas é devido a KIRCHHOFF (1850), que desenvolveu a chamada Teoria Clássica, que representa muito bem o comportamento de placas delgadas sob ação de carregamento transversal e onde o problema é representado por uma equação diferencial de quarta ordem.

REISSNER (1944, 1945) e MINDLIN (1951) desenvolveram teorias semelhantes, chegando a uma equação de sexta ordem, que consideram as deformações por cisalhamento transversal e que permitem a análise de placas delgadas e moderadamente espessas.

HENCKY (1947) e KROMM (1953) desenvolveram teorias envolvendo os deslocamentos da superfície média da placa e as rotações neste plano. 
Mais recentemente, CHENG (1979) desenvolveu uma teoria, obtendo uma equação diferencial de ordem infinita para os deslocamentos transversais, onde derivadas maiores que as de quarta ordem multiplicam os quadrados da espessura da placa. No limite, quando a espessura tende a zero, obtém-se a equação bi-harmônica da Teoria Clássica.

LEVINSON (1980) deduziu uma nova teoria, também considerando as deformações por cisalhamento, permitindo a análise estática e dinâmica de placas.

Em trabalho recente, REISSNER (1986) apresentou nova formulação, generalizando as equações para a análise de placas considerando-se grandes deformações e obtendo um sistema de equações diferenciais de décima ordem. Do mesmo autor, o trabalho de 1991 aborda a análise de placas ortotrópicas.

Finalmente, BARRET \& ELLIS (1988) desenvolveram uma extensão da teoria de Cheng, obtendo as expressões das componentes de deslocamentos e tensões em termos do deslocamento transversal da superfície média da placa e suas derivadas.

O Método dos Elementos de Contorno (MEC), cuja formulação é baseada em equações integrais, surgiu há apenas 30 anos. Porém, desde o início do século, a partir do trabalho de FREDHOLM (1903), as equações integrais são utilizadas para a solução de alguns problemas físicos particulares. Nos anos sessenta surge a primeira formulação indireta do método dos elementos de contorno, embora ainda não tendo essa denominação, de autoria de KUPRADZE (1965), aplicado a problemas potenciais e elásticos.

Somente a partir de 1967, com a publicação do primeiro artigo sobre a formulação direta do método das equações integrais de contorno, para problemas elásticos bidimensionais, de autoria de Frank J. RIZZO (1967), é que os métodos integrais começam a despertar interesse na comunidade científica.

A generalização do método para sua utilização ampla em problemas de engenharia ocorre em 1975, com o trabalho de LACHAT (1975), quando as técnicas de resolução das equações integrais começam a ser vistas como métodos numéricos.

O método passa a ser conhecido como "Método dos Elementos de Contorno", com a publicação do primeiro livro sobre o método pelo professor Carlos A. BREBBIA (1978), onde o autor formula o método a partir do método dos resíduos ponderados, usando uma função ponderada conveniente.

A análise de placas, por meio de equações integrais, teve como marco inicial o trabalho de JASWON et al. (1967), onde os autores propõe a solução de uma equação biharmônica. Bem mais tarde, HANSEN (1976) propõe uma formulação para análise de placas infinitas com buracos de contornos não carregados, utilizando-se duas equações integrais: as 
representações integrais dos deslocamentos e de sua derivada. ALTIERO \& SIKARSKIE (1978) analisaram placas engastadas, considerando-se a placa real contida em uma outra de maiores dimensões para a qual era possível estabelecer a função de Green. Essa técnica foi mais tarde generalizada para quaisquer condições de contorno por WU \& ALTIERO (1979). A formulação direta para flexão de placas foi consolidada a partir de 1978. Os trabalhos mais relevantes desta época são os de BÉZINE (1978), TOTTENHAN (1979) e STERN (1979, 1983), onde utilizam-se as representações integrais de deslocamento e de rotação e como problema auxiliar, a solução fundamental.

A partir da formulação direta foram desenvolvidos vários outros trabalhos que generalizaram a formulação do MEC em placas para diversos tipos de análises: BÉZINE (1980) propôs uma formulação mista para análise de vibrações; KAMIYA et al. (1982), aplicaram o modelo a problemas sujeitos a variação de temperatura; VAN DER WEEËN (1982) desenvolveu a formulação para placas espessas com três graus de liberdade por nó de contorno e três representações integrais: uma do deslocamento transversal e duas das rotações, considerando as hipóteses de Reissner; COSTA \& BREBBIA (1985) e também BÉZINE et al. (1985) estudaram placas sujeitas a instabilidade utilizando células internas para integrar o termo de domínio; KATSIKADELIS \& ARMENAKAS (1984) apresentaram formulações para analisar o problema de placas sobre fundação elástica.

Os primeiros trabalhos que trataram placas no contexto de estruturas de pisos de edifícios, foram os de BÉZINE (1981) e HARTMANN \& ZOTEMANTEL (1986), nos quais a simulação de apoios de lajes de edifícios foi feita através da imposição de condições de domínio. Em São Carlos foram diversos os trabalhos desenvolvidos nessa área. O primeiro foi em 1987, quando Prof. João Batista de PAIVA (1987) mostrou diversas associações de placas com estruturas de barras, pilares e vigas. Além deste, destacam-se também os trabalhos de mestrado de SILVA (1988), que estudou placas sobre fundação elástica; CALDERÓN (1991) que tratou do mesma tema e introduziu aproximações alternativas para a integral de domínio; ainda sobre o mesmo tema, o trabalho de MANZOLI (1992) que considerou solução fundamental própria; OLIVEIRA NETO (1991) analisou um procedimento para a melhoria de valores de contorno. Pode-se citar, ainda, o trabalho de CHAVES (1997), que desenvolveu a formulação de placas delgadas, com espessura variável, considerando a Teoria Clássica de Kirchoff. Quatro teses de doutorado também foram finalizadas: RIBEIRO (1992) desenvolveu a formulação de placas com as hipóteses de Reissner considerando não-linearidade física; CHUEIRI (1994) implementou a formulação elasto-plástica, com modelos particulares de concreto armado, considerando-se as hipóteses de Kirchhoff; SILVA (1996) desenvolveu uma formulação de placas com enrijecedores 
utilizando a teoria de Reissner e CALDERÓN (1996), que desenvolveu um estudo sobre a interação de placas com o meio contínuo, através do MEC.

Ainda, através do Método dos Elementos de Contorno, MORJARIA \& MUKHERJEE (1980) desenvolveram uma análise não-linear de placas, considerando um modelo viscoplástico e utilizando uma equação diferencial baseada na teoria clássica de Kirchhoff. MOSHAIOV \& VORUS (1986) e KARAM \& TELLES (1992) desenvolveram uma formulação para a análise elastoplástica de placas, usando um esquema incrementaliterativo e considerando momentos fletores plásticos iniciais, sendo que o primeiro trabalho foi baseado na teoria clássica de Kirchhoff e o segundo na teoria de Reissner.

Diversos tipos de modelos constitutivos têm sido utilizados para descrever o comportamento do concreto, como por exemplo, modelos elasto-plásticos, modelos de dano, de fratura e outros. PROENÇA (1988) desenvolveu um estudo detalhado sobre vários modelos constitutivos para o concreto. Nesse trabalho, será utilizado o modelo elastoplástico, onde o critério adotado é o de Von Mises e o modelo de dano de Mazars.

$\mathrm{Na}$ análise de lajes de concreto armado, uma das propostas de generalização do critério de Von Mises para sua utilização em rupturas frágeis foi apresentada por YANG (1980), incluindo a possibilidade de verificação do efeito de Bauschinger. CHANNAKESHAVA et al. (1988) aplicaram a superfície de Von Mises, com modificações, para verificação do concreto, analisando problemas de estado plano, através do Método dos Elementos de Contorno. HU \& SCHNOBRICH (1991) também introduziram uma adaptação do critério para a análise de placas e cascas de concreto, via Método dos Elementos Finitos.

Vários trabalhos considerando modelos baseados na relação momento-curvatura, têm sido apresentados, para a análise de lajes de concreto armado. Nesta linha, pode-se citar os trabalhos de JOFRIET \& MCNEICE (1971), AUFARE et al. (1986) e CORRÊA (1991), sendo que esse último, desenvolveu um trabalho para a análise elastoplástica de pisos de edifícios, através do Método dos Elementos Finitos.

Contudo, no caso de lajes de concreto armado, é mais indicado o uso de modelos estratificados. Assim, considerando esse tipo de modelo, pode-se citar os trabalhos de HAND et al. (1973) e LIN \& SCORDELIS (1975), sendo que nesse último, foi utilizado o critério elastoplástico de Von Mises para a verificação da ruptura do concreto sob compressão biaxial. Ainda nessa linha, pode-se citar os trabalhos de FIGUEIRAS (1983), que considera a resistência do concreto tracionado entre fissuras e a capacidade de transferência do esforço cortante do concreto fissurado por encaixe dos agregados, e HU \& SCHNOBRICH (1991), que considera o efeito de amolecimento do concreto comprimido, através de um modelo no qual adota-se uma regra de fluxo não associativa. 
A Mecânica do Dano Contínuo, que tem como marco inicial o trabalho de KACHANOV (1958), é atualmente uma importante ferramenta utilizada por pesquisadores na análise do comportamento não-linear de estruturas em concreto armado. Nas últimas duas décadas, a Mecânica do Dano Contínuo tem sido aplicada na solução de vários problemas dentro da engenharia, aos quais se incluem a modelação da deterioração lenta do material (creep damage) (KACHANOV (1984) e MURAKAMI (1981)), dano por fadiga (LEMAITRE (1984) e MARIGO (1985)), interação deterioração lenta-fadiga ( LEMAITRE \& CHABOCHE (1974) e LEMAITRE (1984)), dano em materiais dúcteis (SIMO \& JU (1987), TAI (1990) e HAN \& MOU (1993)) e dano em estruturas de concreto armado (MAZARS (1984)).

Em São Carlos foram desenvolvidos alguns trabalhos, que consideram o modelo de dano de MAZARS, como o de ÁLVARES (1993), que faz a análise do modelo para o concreto utilizando o Método dos Elementos Finitos e DRIEMEIER (1995), que estuda o comportamento do concreto sob solicitação cíclica. Pode-se citar, ainda, o trabalho de BUSSAMRA (1993), onde o autor apresenta equações constitutivas para o concreto baseadas na mecânica do dano contínuo.

\subsection{Objetivos e Justificativa}

O objetivo do trabalho proposto é apresentar modelos, quer seja elastoplástico ou de dano, que representem bem o comportamento do concreto armado, para a análise não-linear de flexão simples de placas delgadas de concreto armado, através do Método dos Elementos de Contorno (MEC).

A formulação de placas utilizada é baseada na teoria de Kirchoff, onde a solução não-linear do problema é obtida considerando-se um modelo baseado no processo dos momentos iniciais, utilizando-se o Método de Newton-Raphson Modificado.

A distribuição não-linear das tensões ao longo da espessura da placa, é obtida através de um modelo estratificado, onde se impõe que a força normal resultante no concreto armado deve ser nula. Para o concreto, serão considerados dois modelos bi-dimensionais: o modelo de dano de MAZARS (1984) e o modelo elastoplástico com encruamento isótropo negativo, usando o critério de Von-Mises, onde será considerado que o concreto tem resistência somente à compressão. Para a armadura será considerado um modelo elastoplástico unidimensional com encruamento isótropo positivo.

A integração numérica será feita através da fórmula da quadratura de Gauss, onde será usada a técnica de sub-elementos, a fim de se obter uma melhor precisão nos resultados. 
Os elementos do contorno serão lineares, sendo que os deslocamentos e esforços nos mesmos serão aproximados por uma função polinomial do segundo grau. Os momentos iniciais no contorno e no domínio da placa serão aproximados por funções lineares definidas em células internas.

Este trabalho representa o início de um programa mais completo para a análise de pavimento de edifícios, tabuleiro de pontes e outros, sendo suficiente para tal a imposição de condições internas ao elemento de placa e a associação do mesmo a outros elementos estruturais através do acoplamento com o Método dos Elementos Finitos.

O Método dos Elementos de Contorno apresenta um bom desempenho para a análise de placas. Neste particular, a precisão que mostra é ainda mais notada em pontos de concentração de esforços. Logo, o método se apresenta com uma característica importante para a determinação de esforços em uma estrutura de pisos de edifícios, que usualmente apresentam diversos carregamentos em áreas de pequenas dimensões, como é o caso dos pilares, e também carregamentos em linha, no caso de vigas cujas reações são linha de cargas e de momentos aplicados.

Deve-se notar que as representações integrais de momentos e forças cortantes são exatas; o erro introduzido é devido à aproximação dos valores de contorno. Assim, momentos e forças cortantes são obtidos com a mesma ordem de erros dos deslocamentos e rotações.

A implementação em microcomputador da formulação proposta será feita utilizandose a linguagem FORTRAN Power Station versão 4.0.

\subsection{Conteúdo do Trabalho}

No capítulo 2 são apresentadas as hipóteses básicas da teoria de Kirchhoff, assim como a expressão dos deslocamentos e as relações elásticas entre tensão e deformação que resultam das mesmas. No mesmo capítulo, tem-se ainda, as expressões dos esforços, das relações de equilíbrio e a equação diferencial de placas.

No capítulo 3 são obtidas a equação integral de um ponto do domínio da placa e a de um ponto do contorno. É apresentado também nesse capítulo, a transformação das integrais de domínio que envolvem o carregamento da placa em equações de contorno, assim como as soluções fundamentais dos deslocamentos e esforços de placa, que são utilizados na formulação. 
No capítulo 4 as equações integrais são transformadas em equações algébricas, através da discretização do contorno em elementos, sobre os quais os deslocamentos e esforços são aproximados por funções aproximadoras. Além das equações algébricas dos deslocamentos, obtêm-se as equações algébricas dos esforços para os pontos do domínio e do contorno. Têm-se, ainda, nesse capítulo, alguns exemplos numéricos, onde obtêm-se a solução linear de placas sujeitas a carregamentos transversais.

No capítulo 5 é apresentada a formulação de placas considerando-se um campo de momentos iniciais, onde são deduzidas as equações integrais e algébricas dos deslocamentos e esforços para os pontos do domínio e do contorno. Os momentos iniciais são aproximados no domínio, através da discretização do mesmo em células. Essa será a formulação utilizada para se obter a solução não-linear de placas sujeitas à carregamentos transversais.

No capítulo 6 são apresentados os modelos constitutivos utilizados para o concreto e a armadura. No caso do concreto, adotaram-se dois modelos bidimensionais: um elastoplástico e outro de dano e para a armadura, adotou-se um modelo elasto-plástico unidimensional.

No capítulo 7 é apresentada a solução não-linear do problema, assim como o modelo estratificado utilizado na integração das tensões ao longo da espessura da placa e o processo para zerar a normal resultante numa seção da placa. No mesmo capítulo, são apresentados alguns exemplos numéricos da formulação não-linear proposta.

No capítulo 8 são feitas as conclusões. 


\section{TEORIA DE PLACAS DELGADAS}

\subsection{Introdução}

Placa é um elemento estrutural caracterizado por apresentar duas das três dimensões muito grandes em comparação com a terceira e cujo carregamento é transversal à sua superfície. Assim, a representação geométrica adotada para a placa é bidimensional, sendo os eixos cartesianos $\mathbf{x}_{\mathbf{1}} \mathbf{x}_{\mathbf{2}}$ definidos em sua superfície média, como mostra a figura (2.1), no caso de uma placa retangular:

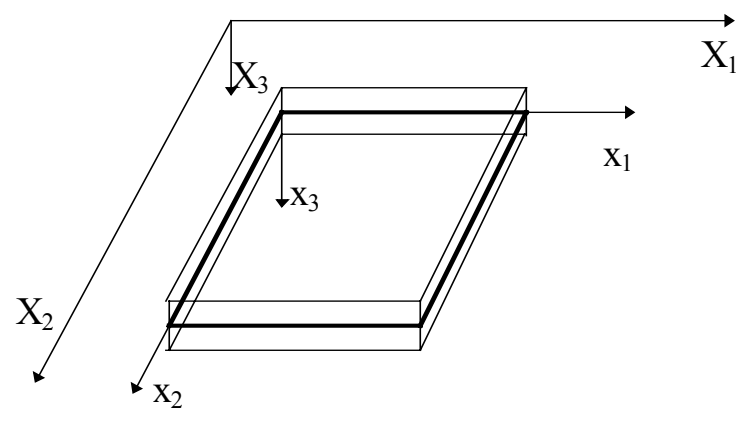

FIGURA 2.1 - Elemento Bidimensional de Placa

Cada ponto ao longo da espessura da placa é definido pela cota $\mathbf{x}_{\mathbf{3}}$, onde

$-\frac{t}{2} \leq x_{3} \leq \frac{t}{2}$. 
A placa é considerada sujeita à flexão simples, isto é, ela não suporta forças normais, sendo submetida apenas à cargas transversais, ou seja, paralelas à $\mathbf{x}_{3}$.

Para a construção do modelo de placas utilizado nesse trabalho, utilizar-se-á a Teoria de Kirchhoff, que interpreta bem o problema de placas delgadas com pequenos deslocamentos, e é construído a partir de algumas hipóteses básicas, as quais são mostradas a seguir:

\section{- Hipóteses}

- Os deslocamentos transversais são pequenos quando comparados com a espessura $\mathbf{t}$ da placa;

- Não há deformação da superfície média da placa, sob o efeito de cargas transversais;

- Os pontos situados inicialmente em uma normal à superfície média da placa permanecem sobre essa normal depois da flexão. Isso equivale à dizer que as seções transversais permanecem planas após a flexão, sofrendo apenas rotação em relação aos eixos neutros. Assim, o esforço cortante não têm influência sobre a flecha, que ocorre unicamente devido à flexão.

- As tensões normais na direção transversal da placa ( $\sigma_{\times 3}$ ), são desprezadas.

A terceira hipótese permite desprezar as deformações de cisalhamento transversal, ou seja: $\gamma_{23}=\gamma_{13}=\mathbf{0}$ e portanto, conclui-se que as componentes de tensão $\tau_{23}$ e $\tau_{13}$ também são nulas. A deformação $\varepsilon_{3}$ não será considerada na formulação do problema, pois como a componente de tensão $\sigma_{3}$ é desprezada, o produto $\sigma_{3}$. $\varepsilon_{3}$, que aparece na equação (3.2) será sempre nulo.

\subsection{Relações Básicas}

\subsubsection{Deslocamentos}

Considera-se que as componentes do vetor deslocamento de um ponto qualquer da placa são dadas pelos deslocamentos $\mathbf{u}_{1}, \mathbf{u}_{2}, \mathbf{u}_{3}$, respectivamente nas direções $\mathbf{X}_{\mathbf{1}}, \mathbf{X}_{\mathbf{2}}, \mathbf{X}_{\mathbf{3}}$ e pelas rotações $\theta \mathbf{x}_{1}$ e $\theta \mathbf{x}_{2}$ em relação às direções $\mathbf{X}_{1}$ e $\mathbf{X}_{2}$. Quando o ponto pertence à superfície média da placa, tem-se que: 


$$
\mathbf{u}_{1}=\mathbf{u}_{2}=\mathbf{0} \quad \text { e } \quad \mathbf{u}_{3}=\mathbf{w} \neq \mathbf{0}
$$

sendo que o deslocamento $\mathbf{u}_{3}$ é denominado de flecha $\mathbf{w}$.

Assim, como a placa é definida pela superfície média, apenas a flecha w fará parte da formulação do problema. Contudo, para pontos que não pertencem à superfície média, a terceira hipótese permite conhecer $\mathbf{u}_{1}$ e $\mathbf{u}_{2}$ a partir de $\mathbf{w}\left(\mathbf{x}_{1}, \mathbf{x}_{2}\right)$, como mostra as figuras abaixo, onde estão indicados os sentidos positivos de $\alpha_{1}$ e $\alpha_{2}$, segundo a regra da mão direita.
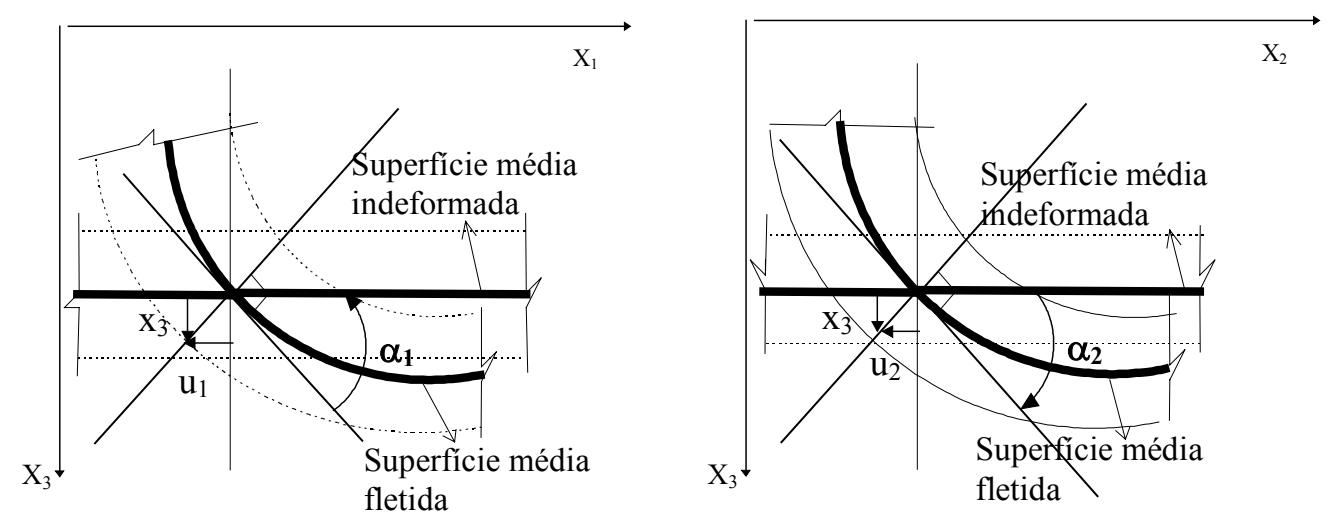

FIGURA 2.2 - Deslocamentos $\mathbf{u}_{1}$ e $\mathbf{u}_{2}$ de um ponto de cota $\mathbf{x}_{3}$

onde $\alpha_{1}=\theta \mathbf{x}_{2}=-\frac{\partial \mathbf{w}}{\partial \mathbf{x}_{1}}$ é a inclinação em $\mathbf{x}_{1}$ da deformada, ou seja, a rotação em torno de $\mathbf{x}_{2}$ e $\alpha_{2}=\theta \mathbf{x}_{1}=\frac{\partial \mathbf{w}}{\partial \mathbf{x}_{2}}$ é a inclinação em $\mathbf{x}_{2}$ da deformada, ou seja, a rotação em torno de $\mathbf{x}_{1}$.

Desse modo, de acordo com a figura (2.2), os deslocamentos $\mathbf{u}_{\mathbf{1}}$ e $\mathbf{u}_{\mathbf{2}}$, de um ponto de $\operatorname{cota} \mathbf{x}_{3}$, são dados por:

$$
\begin{aligned}
& u_{1}=x_{3} \operatorname{tg} \alpha_{1} \\
& u_{2}=-x_{3} \operatorname{tg} \alpha_{2}
\end{aligned}
$$

Considerando-se que o valor de $\mathbf{w}$ é pequeno, os ângulos $\alpha_{i}$ se confundem com o valor de suas tangentes e portanto, na forma indicial, os deslocamentos, dados pelas equações (2.1), resultam em: 


$$
\mathbf{u}_{\mathbf{i}}=-\mathbf{x}_{\mathbf{3}} \mathbf{w},_{\mathbf{i}} \quad(\mathrm{i}=1,2)
$$

\subsubsection{Deformações}

Considerando-se as hipóteses feitas no item (2.1), o tensor de deformações é dado por:

$$
\{\varepsilon\}=\left\{\begin{array}{c}
\varepsilon_{1} \\
\varepsilon_{2} \\
\gamma_{12}
\end{array}\right\}=\left\{\begin{array}{c}
\frac{\partial \mathbf{u}_{1}}{\partial \mathbf{x}_{1}} \\
\frac{\partial \mathbf{u}_{2}}{\partial \mathbf{x}_{2}} \\
\left(\frac{\partial \mathbf{u}_{1}}{\partial \mathbf{x}_{2}}+\frac{\partial \mathbf{u}_{2}}{\partial \mathbf{x}_{1}}\right)
\end{array}\right\}
$$

Considerando-se a equação (2.2), o tensor de deformações é dado, na forma explícita, por:

$$
\{\varepsilon\}=\left\{\begin{array}{c}
\varepsilon_{11} \\
\varepsilon_{22} \\
\gamma_{12}
\end{array}\right\}=\left\{\begin{array}{c}
-\mathbf{x}_{3} \frac{\partial^{2} \mathbf{w}}{\partial \mathbf{x}_{1}^{2}} \\
-\mathbf{x}_{3} \frac{\partial^{2} \mathbf{w}}{\partial \mathbf{x}_{2}^{2}} \\
-2 \mathbf{x}_{3} \frac{\partial^{2} \mathbf{w}}{\partial \mathbf{x}_{1} \partial \mathbf{x}_{2}}
\end{array}\right\}
$$

ou na forma indicial:

$$
\varepsilon_{\mathrm{ij}}=-\mathbf{x}_{3} \mathbf{w},_{\mathrm{ij}} \quad(\mathrm{i}, \mathrm{j}=1,2)
$$

onde:

- $\quad \mathbf{w}, \mathrm{ij}$ é o tensor das curvaturas, sendo $\mathrm{w},{ }_{11}$ sua componente num plano paralelo ao $\mathrm{x}_{1} \mathrm{x}_{3} \mathrm{e}$ $\mathrm{W}, 22$ sua componente num plano paralelo ao $\mathrm{x}_{2} \mathrm{x}_{3}$,

- $\varepsilon_{12}=\frac{\gamma_{12}}{2}$

\subsubsection{Tensões}


Considerando-se as hipóteses feitas no item (2.1), tem-se um caso de estado plano de tensão o qual, considerando-se a lei de Hooke, é dado por:

$$
\{\sigma\}=\left\{\begin{array}{l}
\sigma_{11} \\
\sigma_{22} \\
\tau_{12}
\end{array}\right\}=\frac{\mathbf{E}}{1-v^{2}}\left[\begin{array}{ccc}
1 & v & \mathbf{0} \\
v & 1 & \mathbf{0} \\
\mathbf{0} & 0 & \frac{1-v}{2}
\end{array}\right]\left\{\begin{array}{l}
\varepsilon_{11} \\
\varepsilon_{22} \\
\gamma_{12}
\end{array}\right\}
$$

ou indicialmente:

$$
\sigma_{\mathrm{ij}}=\mathbf{2 G} \varepsilon_{\mathrm{ij}}+\frac{\mathbf{2 G} v}{1-v} \varepsilon_{\mathrm{kk}} \delta_{\mathrm{ij}} \quad(\mathrm{i}, \mathrm{j}, \mathrm{k}=1,2)
$$

onde: $\quad \mathbf{G}=\frac{\mathbf{E}}{\mathbf{2 ( 1 + v )}} \quad$ é o módulo de elasticidade transversal do material da placa,

$$
\begin{aligned}
& v \quad \text { é o coeficiente de Poisson do material, } \\
& \text { E é o módulo de elasticidade longitudinal do material. }
\end{aligned}
$$

Substituindo-se (2.4b) em (2.6), obtém-se:

$$
\sigma_{\mathrm{ij}}=-\frac{\mathbf{E} \mathbf{x}_{3}}{\left(1-v^{2}\right)}\left[v \mathbf{w},_{k \mathbf{k}} \delta_{\mathrm{ij}}+(\mathbf{1}-v) \mathbf{w},_{\mathrm{ij}}\right] \quad(\mathrm{i}, \mathrm{j}, \mathrm{k}=1,2)
$$

\subsubsection{Esforços e Relações de Equilíbrio}

As tensões que agem em um elemento de placa de dimensões $\left(\mathrm{dx}_{1}, \mathrm{dx}_{2}, \mathrm{dx}_{3}\right)$ estão indicadas na figura (2.3). As tensões $\sigma_{\mathrm{ii}}$ e $\tau_{\mathrm{ij}}$ (com $\mathrm{i}, \mathrm{j}=1,2$ ), causam, respectivamente, os momentos $\mathbf{M}_{\mathbf{i i}}$ e $\mathbf{M}_{\mathbf{i j}}$ e a tensão $\tau_{\mathbf{i 3}}$ (com $\mathrm{i}=1,2$ ), os esforços cortantes. Desse modo, os esforços, no sentido positivo, que atuam no plano médio do elemento estão representados na figura (2.4). 

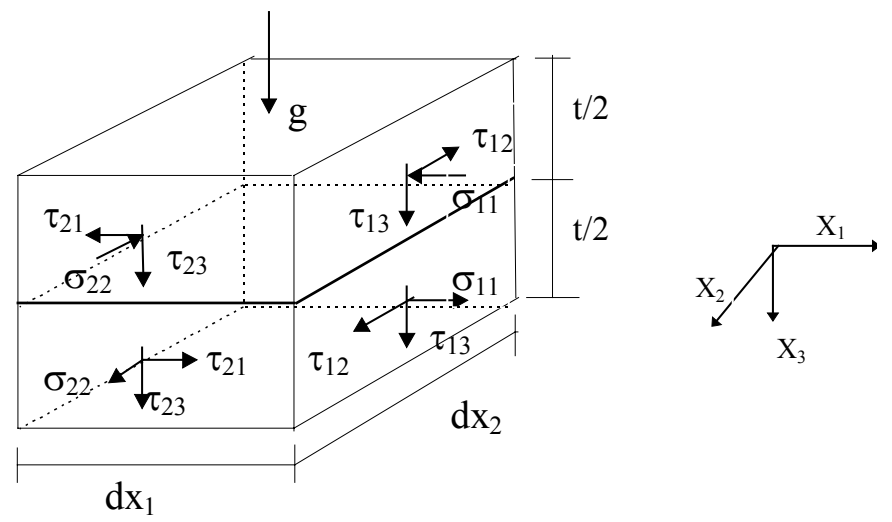

FIGURA 2.3 - Tensões em um Elemento de Placa
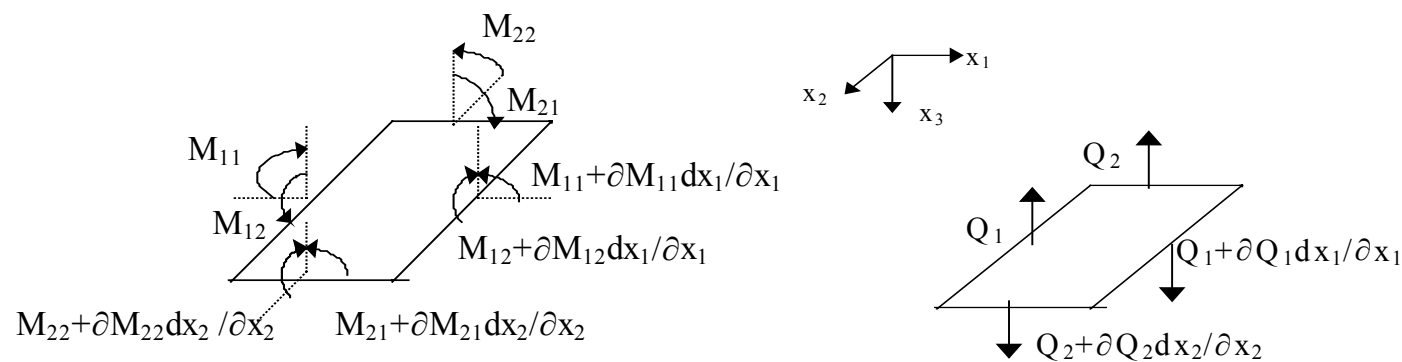

FIGURA 2.4 - Esforços em um Elemento de Placa

Na figura (2.4) $\mathbf{M}_{11}$ e $\mathbf{M}_{22}$, momentos de flexão, e $\mathbf{M}_{12}$ e $\mathbf{M}_{21}$, momentos volventes, são momentos por unidade de comprimento e são obtidos integrando-se as tensões ao longo da espessura $\mathbf{x}_{3}$, considerando-se a equação (2.7), como é mostrado a seguir:

$$
\mathbf{M}_{11}=\int_{-\frac{t}{2}}^{\frac{t}{2}} \sigma_{11} \mathbf{x}_{3} \mathbf{d} \mathbf{x}_{3}=-\frac{\mathbf{E}}{1-v^{2}}\left(\frac{\partial^{2} \mathbf{w}}{\partial \mathbf{x}^{2}}+v \frac{\partial^{2} \mathbf{w}}{\partial \mathbf{y}^{2}}\right) \int_{-\frac{\mathrm{t}}{2}}^{\frac{\mathrm{t}}{2}} \mathbf{x}_{3}^{2} \mathbf{d} \mathbf{x}_{3}=-\mathbf{D}\left(\frac{\partial^{2} \mathbf{w}}{\partial \mathbf{x}_{1}^{2}}+v \frac{\partial^{2} \mathbf{w}}{\partial \mathbf{x}_{2}^{2}}\right)
$$

onde $\mathbf{D}=\frac{\mathbf{E t}^{3}}{12\left(1-v^{2}\right)}$, representa a rigidez à flexão da placa.

Analogamente, obtém-se $\mathbf{M}_{\mathbf{1 2}}$ e $\mathbf{M}_{\mathbf{2 2}}$. Na forma matricial, tem-se: 


$$
\left\{\begin{array}{l}
\mathbf{M}_{11} \\
\mathbf{M}_{22} \\
\mathbf{M}_{12}
\end{array}\right\}=\frac{\mathbf{E t}^{3}}{12\left(1-v^{2}\right)}\left[\begin{array}{ccc}
1 & v & 0 \\
v & 1 & 0 \\
0 & 0 & \frac{1-v}{2}
\end{array}\right]\left\{\begin{array}{c}
-\frac{\partial^{2} \mathbf{w}}{\partial \mathbf{x}_{1}^{2}} \\
-\frac{\partial^{2} \mathbf{w}}{\partial \mathbf{x}_{2}^{2}} \\
-2 \frac{\partial^{2} \mathbf{w}}{\partial \mathbf{x}_{1} \partial \mathbf{x}_{2}}
\end{array}\right\}
$$

ou na forma indicial:

$$
\mathbf{M}_{\mathrm{ij}}=-\mathbf{D}\left[v \mathbf{w},{ }_{\mathbf{k} \mathbf{k}} \delta_{\mathrm{ij}}+(\mathbf{1}-v) \mathbf{w},_{\mathrm{ij}}\right] \quad(\mathrm{i}, \mathrm{j}, \mathrm{k}=1,2)
$$

Invertendo a equação (2.8), obtêm-se as curvaturas, a partir dos momentos:

$$
\left\{\begin{array}{c}
-\frac{\partial^{2} \mathbf{w}}{\partial \mathbf{x}_{1}^{2}} \\
-\frac{\partial^{2} \mathbf{w}}{\partial \mathbf{x}_{2}^{2}} \\
-2 \frac{\partial^{2} \mathbf{w}}{\partial \mathbf{x}_{1} \partial \mathbf{x}_{2}}
\end{array}\right\}=\frac{12}{\mathbf{t}^{3} \mathbf{E}}\left[\begin{array}{ccc}
1 & -v & 0 \\
-v & 1 & 0 \\
0 & 0 & 2(1+v)
\end{array}\right]\left\{\begin{array}{l}
\mathbf{M}_{11} \\
\mathbf{M}_{22} \\
\mathbf{M}_{12}
\end{array}\right\}
$$

Os esforços cortantes, dados por unidade de comprimento, são obtidos a partir de:

$$
Q_{1}=\int_{-\frac{t}{2}}^{\frac{t}{2}} \tau_{13} d x_{3}
$$$$
Q_{2}=\int_{-\frac{t}{2}}^{\frac{t}{2}} \tau_{23} d x_{3}
$$

Contudo, como as componentes de tensão $\tau_{23}$ e $\tau_{13}$ são desprezadas, os esforços cortantes serão obtidos através das equações de equilíbrio do elemento, pois apesar dos esforços cortantes produzidos por uma carga transversal terem efeitos desprezíveis sobre a flexão de uma placa delgada, os mesmos não podem ser desprezados nas equações de equilíbrio do elemento. Assim, considerando-se um carregamento distribuído g e fazendo-se o equilíbrio das forças verticais e dos momentos em torno de $\mathbf{x}_{\mathbf{1}}$ e $\mathbf{x}_{2}$, obtêm-se duas relações de equilíbrio, que são dadas a seguir na forma explícita e indicial: 


$$
\frac{\partial \mathbf{Q}_{1}}{\partial \mathbf{x}_{1}}+\frac{\partial \mathbf{Q}_{2}}{\partial \mathbf{x}_{2}}+\mathbf{g}=\mathbf{0}
$$

ou

$$
\begin{gathered}
\mathbf{Q}_{\mathbf{i}},_{\mathbf{i}}+\mathbf{g}=\mathbf{0} \\
\frac{\partial \mathbf{M}_{12}}{\partial \mathbf{x}_{1}}+\frac{\partial \mathbf{M}_{21}}{\partial \mathbf{x}_{2}}+\frac{\partial \mathbf{M}_{22}}{\partial \mathbf{x}_{2}}+\frac{\partial \mathbf{M}_{11}}{\partial \mathbf{x}_{1}}-\mathbf{Q}_{1}-\mathbf{Q}_{2}=\mathbf{0}
\end{gathered}
$$

$\mathrm{ou}$

$$
\mathbf{M}_{\mathrm{ij}}, \boldsymbol{i}_{\mathrm{i}}-\mathbf{Q}_{\mathbf{j}}=\mathbf{0} \quad(\mathrm{i}, \mathrm{j}=1,2)
$$

Logo, a partir das equações (2.9) e (2.12.b), obtém-se:

$$
\mathbf{Q}_{\mathbf{j}}=\mathbf{M}_{\mathrm{ij}, \mathrm{i}}=-\mathbf{D} \mathbf{w}_{\mathbf{k} \mathbf{k j}} \quad(\mathrm{i}, \mathrm{j}, \mathrm{k}=1,2)
$$

\subsubsection{Equação Diferencial de Placas}

Diferenciando-se a equação (2.12.b) em relação à $\mathbf{x}_{\mathbf{j}}$ e considerando a equação (2.11.b), obtém-se a equação diferencial de placas em função dos momentos:

$$
\mathbf{M}_{\mathrm{ij}},{ }_{\mathrm{ij}}+\mathbf{g}=\mathbf{0} \quad(\mathrm{i}, \mathrm{j}=1,2)
$$

Derivando-se (2.13) e considerando-se (2.14), chega-se à equação diferencial de placas em função dos deslocamentos transversais:

$$
\mathbf{w}_{\text {,kkll }}=\frac{\mathbf{g}}{\mathbf{D}} \quad(\mathrm{k}, 1=1,2)
$$

Na forma explícita:

$$
\frac{\partial^{4} \mathbf{w}}{\partial \mathbf{x}_{1}^{4}}+2 \frac{\partial^{4} \mathbf{w}}{\partial \mathbf{x}_{1}^{2} \partial \mathbf{x}_{2}^{2}}+\frac{\partial^{4} \mathbf{w}}{\partial \mathbf{x}_{2}^{4}}=\frac{\mathbf{g}}{\mathbf{D}}
$$


ou ainda: $\nabla^{2} \nabla^{2} \mathbf{w}=\frac{\mathbf{g}}{\mathbf{D}}$

onde $\nabla^{2}=\frac{\partial^{2}}{\partial \mathbf{x}_{\mathbf{k}} \partial \mathbf{x}_{\mathbf{k}}}=\left(\frac{\partial^{2}}{\partial \mathbf{x}_{1} \partial \mathbf{x}_{1}}+\frac{\partial^{2}}{\partial \mathbf{x}_{2} \partial \mathbf{x}_{2}}\right)$ é o operador de Laplace.

\subsection{Esforços Segundo um Sistema de Coordenadas Genérico (n,s)}

Nos problemas de placas, as incógnitas do contorno são calculadas segundo o sistema de coordenadas (n,s), sendo $\mathbf{n}$ a direção normal ao contorno e s a direção tangencial ao mesmo. Portanto, é preciso também conhecer os esforços em relação a um sistema genérico de coordenadas n e s. Assim, seja o plano médio de um elemento de placa abc, onde o sistema ns é adotado na face inclinada do mesmo:
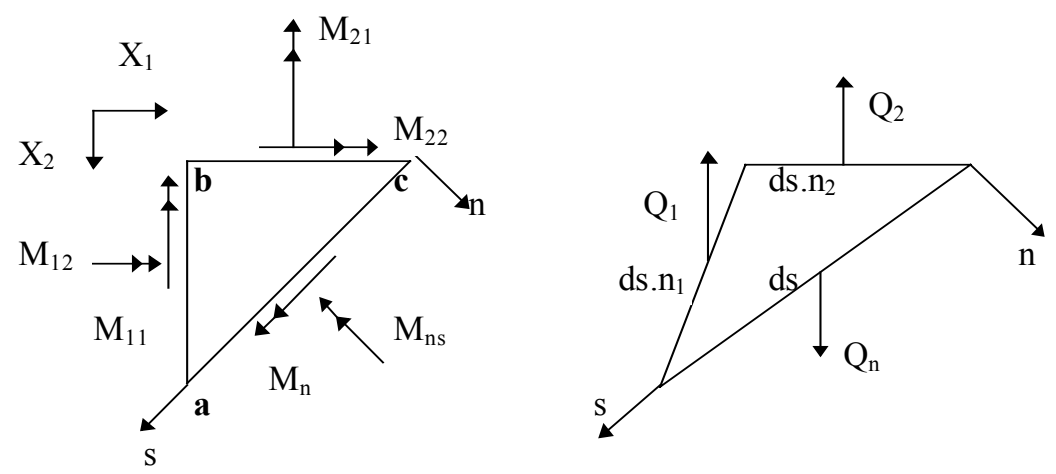

FIGURA 2.5 - Esforços no Elemento abc

Os momentos, nas direções $\mathbf{x}_{\mathbf{1}}$ e $\mathbf{x}_{\mathbf{2}}$, que atuam na face de normal $\mathbf{n}$ são dados por:

$$
\left\{M_{n}\right\}=\left\{\begin{array}{l}
M_{n 1} \\
M_{n 2}
\end{array}\right\}=\left[\begin{array}{ll}
M_{11} & M_{12} \\
M_{21} & M_{22}
\end{array}\right]\left\{\begin{array}{l}
n_{1} \\
n_{2}
\end{array}\right\}=\left\{\begin{array}{l}
M_{11} n_{1}+M_{12} n_{2} \\
M_{21} n_{1}+M_{22} n_{2}
\end{array}\right\}
$$

onde $\mathbf{n}_{\mathbf{j}}$ são os cossenos diretores da direção $\mathbf{n}$

Fazendo-se a rotação de eixos, obtêm-se a componente de momento $\mathbf{M}_{\mathbf{n}}$ na direção $\mathbf{n}$ e a componente de momento $\mathbf{M}_{\mathbf{n s}}$ na direção $\mathbf{s}$, a partir do vetor de momentos $\mathbf{M}_{\mathbf{n}}$, dado pela equação (2.16). Logo, têm-se: 


$$
\begin{aligned}
& M_{n n}=\left\{\begin{array}{ll}
n_{1} & n_{2}
\end{array}\right\}\left\{\begin{array}{l}
M_{n 1} \\
M_{n 2}
\end{array}\right\}=M_{11} n_{1} n_{1}+M_{12} n_{1} n_{2}+M_{21} n_{1} n_{2}+M_{22} n_{2} n_{2} \\
& M_{n s}=\left\{\begin{array}{ll}
s_{1} & s_{2}
\end{array}\right\}\left\{\begin{array}{l}
M_{n 1} \\
M_{n 2}
\end{array}\right\}=M_{11} n_{1} s_{1}+M_{12} s_{1} n_{2}+M_{21} n_{1} s_{2}+M_{22} s_{2} n_{2}
\end{aligned}
$$

onde $\mathbf{s}_{\mathbf{j}}$ são os cossenos diretores da direção $\mathbf{s}$.

Indicialmente, têm-se:

$$
\begin{aligned}
& \mathbf{M}_{\mathrm{nn}}=\mathbf{M}_{\mathrm{ij}} \mathbf{n}_{\mathrm{i}} \mathbf{n}_{\mathrm{j}} \quad(\mathrm{i}, \mathrm{j}=1,2) \\
& \mathbf{M}_{\mathrm{ns}}=\mathbf{M}_{\mathrm{ij}} \mathbf{n}_{\mathrm{i}} \mathbf{s}_{\mathbf{j}}
\end{aligned}
$$

Fazendo-se o equilíbrio das forças verticais, obtém-se:

$$
\mathbf{Q}_{\mathbf{n}}=\mathbf{Q}_{\mathbf{i}} \mathbf{n}_{\mathbf{i}}
$$

\subsubsection{Força Cortante Equivalente e Reação de Canto}

Para a solução da equação diferencial de placas (2.15), é necessário que sejam satisfeitas as condições de contorno do problema. Nos problemas usuais, estas se referem às cinco seguintes variáveis: o deslocamento transversal $\mathbf{w}$ da superfície média, a sua derivada $\partial \mathbf{w} / \partial \mathbf{n}$ e aos esforços $\mathbf{M}_{\mathbf{n}}, \mathbf{M}_{\mathbf{n}}$, e $\mathbf{Q}_{\mathbf{n}}$ dos pontos do contorno da placa, segundo as direções normal e tangencial à borda. Como a equação diferencial é de quarta ordem, pode-se ter apenas quatro variáveis, das quais duas devem ser dadas como condição de contorno, isto é, devem ter seus valores prescritos. Assim, a fim de eliminar uma variável, KIRCHHOFF (1850) demonstrou que as condições de contorno relativas à força cortante $\mathbf{Q}_{\mathbf{n}}$ e ao momento $\mathbf{M}_{\mathbf{n} \text { s }}$ podem ser agrupadas em uma única condição, relativa a um esforço $\mathbf{V}_{\mathbf{n}}$, que é denominado força cortante equivalente.

Assim, seja um ponto $\mathbf{P}$ pertencente ao contorno da placa, conforme a figura (2.6), sobre o qual se define um sistema (n, s), nas direções normal e tangencial ao contorno, respectivamente. 


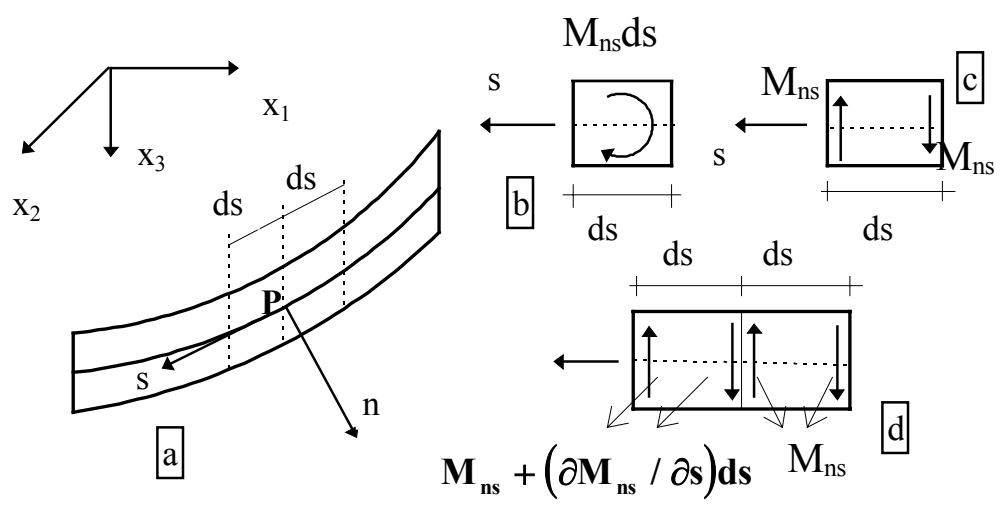

FIGURA 2.6 - Momentos Volventes no Contorno da Placa

Considerem-se, ainda, dois elementos infinitesimais de comprimento ds (figura 2.6.a), onde em cada elemento atua um momento volvente resultante, dado por $\mathbf{M}_{\mathbf{n s}} \mathbf{d s}$ (figura 2.6.b), resultantes das componentes horizontais de tensão na direção s (ver figura 2.3). Esse momento pode ser substituído por duas forças verticais de intensidades iguais a $\mathbf{M}_{\mathbf{n s}}$, aplicadas nas extremidades do elemento, conforme mostra a figura (2.6.c). Essas forças verticais $\mathbf{M}_{\mathrm{ns}}$ serão equilibradas pelas reações verticais do apoio. Assim, tomando-se dois elementos consecutivos (figura 2.6.d), verifica-se que na junção dos elementos existe uma força resultante, dada por $\left(\partial \mathbf{M}_{\mathbf{n s}} / \partial \mathbf{s}\right) \mathbf{d s}$, que faz parte da reação de apoio. A soma desta força com a força cortante no ponto $\mathbf{P}\left(\mathbf{Q}_{\mathbf{n}} \mathbf{d s}\right)$, resulta na força cortante equivalente $\mathbf{V}_{\mathbf{n}} \mathbf{d s}$, cuja intensidade por unidade de comprimento é dada por:

$$
\mathbf{V}_{\mathrm{n}}=\mathbf{Q}_{\mathrm{n}}+\frac{\partial \mathbf{M}_{\mathrm{ns}}}{\partial \mathbf{s}}
$$

Essa substituição de forças não modifica a flexão da placa, pois não altera os valores de $\mathbf{M}_{\mathbf{n s}}$, ela afeta somente a distribuição de tensões na vizinhança do contorno, de acordo com o princípio de St. Venant.

Verifica-se agora, utilizando-se o mesmo esquema estático, o que ocorre em um canto da placa. Considere-se, assim, um canto i qualquer da placa, como é mostrado na figura (2.7), onde $\mathbf{M}_{\text {nsi }}^{+}$e $\mathbf{M}_{\text {nsi }}^{-}$são, respectivamente, os momentos volventes posterior e anterior a esse canto. Como indicado na figura (2.7), uma resultante não nula devido às reações de apoio correspondentes a cada lado, aparece necessariamente no canto. Tal resultante, é denominada reação de canto e é dada por:

$$
\mathbf{R}_{\mathrm{ci}}=\mathbf{M}_{\mathrm{nsi}}^{+}-\mathbf{M}_{\mathrm{nsi}}^{-}
$$




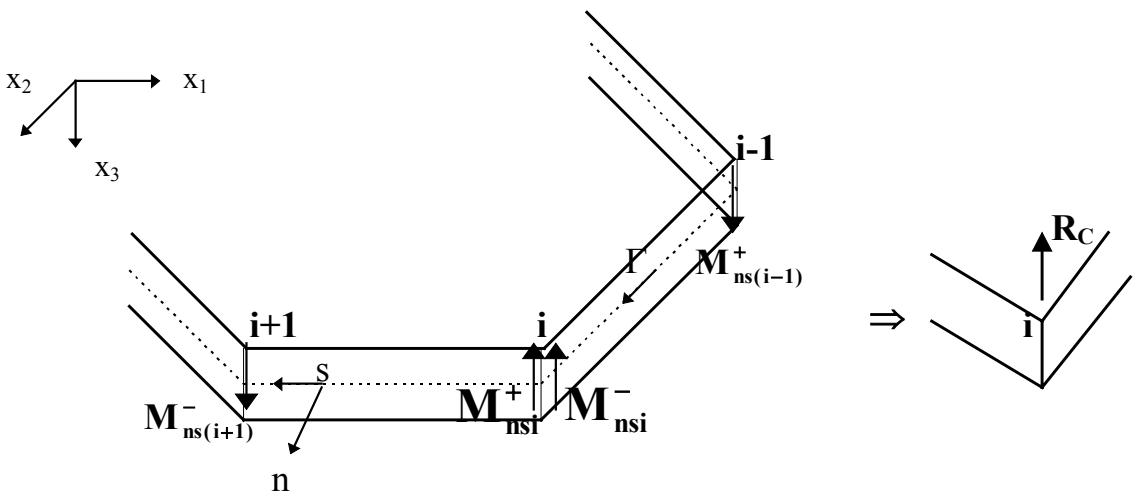

FIGURA 2.7 - Momentos Volventes em um Canto i da Placa

\subsection{Condições de Contorno}

No problema de pacas, de acordo com KIRCHHOFF (1850), têm-se quatro variáveis em cada nó do contorno (ver item 2.2.7): o esforço cortante equivalente $\mathbf{V}_{\mathbf{n}}$, o momento fletor $\mathbf{M}_{\mathbf{n}}$, a flecha $\mathbf{w}$ e a rotação $\partial \mathbf{w} / \partial \mathbf{n}$, das quais duas são conhecidas, pois são impostas como condição de contorno. De um modo geral, quando o deslocamento $\mathbf{U}_{\mathbf{i}}$ é conhecido, o esforço correspondente $\mathbf{P}_{\mathbf{i}}$ é incógnito, e vice-versa, sendo $\mathbf{U}_{\mathbf{i}}$ e $\mathbf{P}_{\mathbf{i}}$ variáveis generalizadas que são dadas por: $\mathbf{U}_{1}=\mathbf{w}, \mathbf{U}_{2}=\partial \mathbf{w} / \partial \mathbf{n}, \mathbf{P}_{1}=\mathbf{V}_{\mathbf{n}}, \mathbf{P}_{2}=\mathbf{M}_{\mathbf{n}}$. Nos casos clássicos de vinculações em problemas de placas (ver figura 2.8), têm-se:

(a) borda engastada: $\mathbf{w}=\partial \mathbf{w} / \partial \mathbf{n}=\mathbf{0}$, e $\mathbf{V}_{\mathbf{n}}$ e $\mathbf{M}_{\mathbf{n}}$ são desconhecidos;

(b) borda simplesmente apoiada: $\mathbf{w}=\mathbf{M}_{\mathbf{n}}=\mathbf{0}$ e $\mathbf{V}_{\mathbf{n}}$ e $\partial \mathbf{w} / \partial \mathbf{n}$ são desconhecidos;

(c) borda livre: $\mathbf{V}_{\mathbf{n}}=\mathbf{M}_{\mathbf{n}}=\mathbf{0}$ e $\mathbf{w}$ e $\partial \mathbf{w} / \partial \mathbf{n}$ são desconhecidos.

Nesse trabalho, consideram-se também os deslocamentos e reações de canto como variáveis do problema, sendo que em cada canto uma delas tem que ser dada como condição de contorno. Assim, no caso do canto pertencer ao contorno, tem-se que $\mathbf{w}_{\mathbf{C}}=\mathbf{0}$ e $\mathbf{R}_{\mathbf{C}}$ é desconhecido. Porém, nos casos de simetria, em que se calcula, por exemplo, somente um quarto da placa, tem-se que $\mathbf{R}_{\mathbf{C}}=\mathbf{0}$ e $\mathbf{w}_{\mathbf{C}}$ é desconhecido para o canto que coincide com um ponto do domínio da placa. 


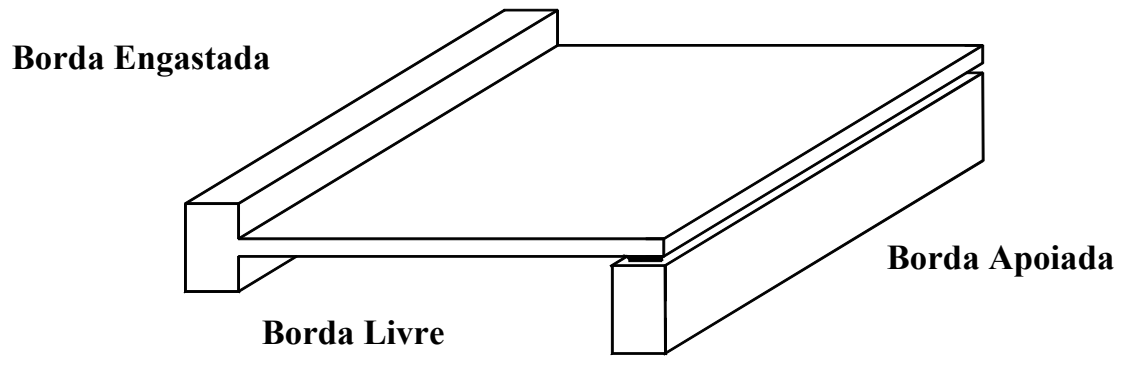

FIGURA 2.8 - Placa com Condições de Contorno.

\subsection{Equação Diferencial e Esforços em Coordenadas Polares}

Esse será o sistema de coordenadas usado na formulação do problema, pois é o mais conveniente para se obter respostas devido a cargas pontuais, que corresponde ao caso do carregamento fundamental. Assim, um ponto $\mathbf{P}$ de coordenadas $\left(\mathrm{x}_{1}, \mathrm{x}_{2}\right)$ pode ser definido em função de $\mathbf{r}$ e $\theta$, que são respectivamente, a distância deste ponto à origem do sistema de coordenadas $\left(\mathrm{x}_{1}, \mathrm{x}_{2}\right)$ e o ângulo entre o segmento $\overline{\mathbf{O P}}$ e o eixo $\mathbf{O \mathbf { x } _ { 1 }}$ (ver figura 2.9).

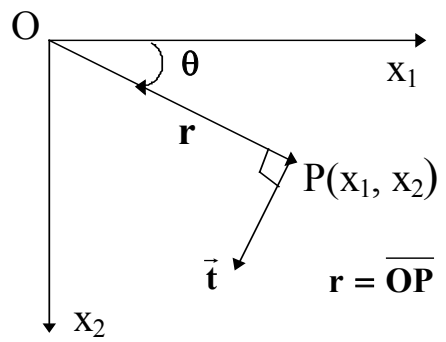

FIGURA 2.9 - Sistema de Coordenadas Polares

onde t é o versor perpendicular à direção de $\mathbf{r}$. Seus cossenos diretores são dados por:

$$
\begin{aligned}
& \mathbf{t}_{1}=-\mathbf{r},,_{2}=-\operatorname{sen} \theta \\
& \mathbf{t}_{2}=\mathbf{r},,_{1}=\cos \theta
\end{aligned}
$$

As equações que relacionam as coordenadas cartesianas às coordenadas polares são:

$$
\begin{aligned}
& x_{1}=r_{1}=r \cos \theta \\
& x_{2}=r_{2}=r \operatorname{sen} \theta
\end{aligned}
$$


As relações inversas são dadas por:

$$
\begin{aligned}
& \mathbf{r}^{2}=\mathbf{x}_{1}^{2}+\mathbf{x}_{2}^{2}=r_{i} \cdot r_{i} \\
& \theta=\operatorname{arctg}\left(\frac{r_{2}}{r_{1}}\right)
\end{aligned}
$$

Derivando-se as equações (2.24), obtêm-se:

$$
\begin{aligned}
& \mathbf{r},_{1}=\frac{\mathbf{r}_{1}}{\mathbf{r}}=\cos \theta \\
& \mathbf{r},_{2}=\frac{\mathbf{r}_{2}}{\mathbf{r}}=\operatorname{sen} \theta \\
& \theta,_{1}=-\frac{\mathbf{r}_{2}}{\mathbf{r}^{2}}=-\frac{\operatorname{sen} \theta}{\mathbf{r}} \\
& \theta,_{2}=\frac{\mathbf{r}_{1}}{\mathbf{r}^{2}}=\frac{\cos \theta}{\mathbf{r}}
\end{aligned}
$$

Derivando-se as equações (2.25) e considerando-se as relações (2.22), obtêm-se:

$$
\begin{aligned}
& \frac{\partial \mathbf{r},_{i}}{\partial \theta}=\mathbf{t}_{\mathbf{i}} \\
& \theta_{,_{i}}=\frac{\mathbf{t}_{\mathbf{i}}}{\mathbf{r}}
\end{aligned}
$$

Considerando-se o deslocamento transversal $\mathbf{w}$, como função de $\mathbf{r}$ e $\theta$, a derivada de $\mathbf{w}(\mathbf{r}, \theta)$ em relação a uma coordenada genérica $\mathbf{x}_{\mathbf{i}}$ é dada por:

$$
\mathbf{w},_{\mathbf{i}}=\frac{\partial \mathbf{w}}{\partial \mathbf{x}_{\mathbf{i}}}=\frac{\partial \mathbf{w}}{\partial \mathbf{r}} \mathbf{r}_{\mathbf{i}}+\frac{\partial \mathbf{w}}{\partial \theta} \theta,_{\mathbf{i}} \quad(\mathrm{i}=1,2)
$$

De onde define-se o operador diferencial de primeira ordem:

$$
\frac{\partial}{\partial \mathbf{x}_{\mathbf{i}}}=\frac{\partial}{\partial \mathbf{r}} \mathbf{r},,_{\mathbf{i}}+\frac{\partial}{\partial \theta} \theta,_{\mathbf{i}}
$$


Desse modo, a segunda derivada pode ser expressa por:

$$
\mathbf{w},_{i j}=\left(\frac{\partial}{\partial \mathbf{r}} \mathbf{r}_{i}+\frac{\partial}{\partial \theta} \theta_{,_{i}}\right)\left(\frac{\partial \mathbf{w}}{\partial \mathbf{r}} \mathbf{r}_{j}+\frac{\partial \mathbf{w}}{\partial \theta} \theta,_{j}\right) \quad(i, j=1,2)
$$

Desenvolvendo-se as derivadas, obtém-se:

$$
\begin{aligned}
\mathbf{w}_{\mathrm{ij}}= & \frac{\partial^{2} \mathbf{w}}{\partial \mathbf{r}^{2}}\left(\mathbf{r},_{\mathrm{i}} \mathbf{r},_{j}\right)+\frac{\partial \mathbf{w}}{\partial \mathbf{r}}\left(\frac{\partial \mathbf{r},_{j}}{\partial \theta} \theta,_{i}\right)+\frac{\partial^{2} \mathbf{w}}{\partial \mathbf{r} \partial \theta}\left(\mathbf{r},_{i} \theta,_{j}+\mathbf{r},,_{j} \theta,_{i}\right)+ \\
& +\frac{\partial^{2} \mathbf{w}}{\partial \theta^{2}}\left(\theta,_{i} \theta,_{j}\right)+\frac{\partial \mathbf{w}}{\partial \theta}\left(\frac{\partial \theta,_{j}}{\partial \mathbf{r}} \mathbf{r},_{i}+\frac{\partial \theta,_{j}}{\partial \mathbf{r}} \theta,_{i}\right)
\end{aligned}
$$

Para os casos em que o problema apresenta simetria em relação à origem do sistema de coordenadas, como ocorre no problema fundamental de placas, o deslocamento $\mathbf{w}$ é função apenas de $\mathbf{r}$, já que não varia com $\theta$. Nesse caso, considerando-se as equações (2.27) e (2.28), $\mathbf{w},{ }_{\mathbf{i j}}$ é dada por:

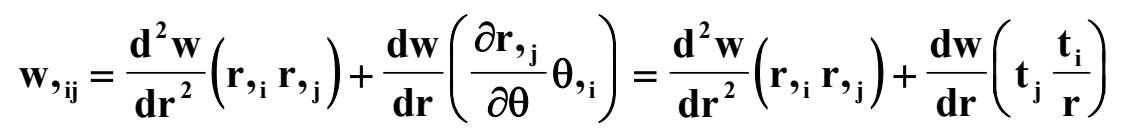

Assim, a partir da equação (2.33), pode-se definir o operador diferencial de segunda ordem como sendo:

$$
\frac{\partial^{2}}{\partial \mathbf{x}_{\mathrm{i}} \partial \mathbf{x}_{\mathrm{j}}}=\left(\mathbf{r},{ }_{\mathrm{i}} \mathbf{r},{ }_{\mathrm{j}}\right) \frac{\mathbf{d}^{2}}{\mathbf{d r ^ { 2 }}}+\frac{1}{\mathbf{r}}\left(\mathbf{t}_{\mathrm{i}} \mathbf{t}_{\mathrm{j}}\right) \frac{\mathbf{d}}{\mathbf{d r}}
$$

Usando índices repetidos em (2.34) e considerando-se $\mathbf{r}, \mathbf{k}, \mathbf{r},{ }_{\mathbf{k}}=\mathbf{t},{ }_{\mathbf{k}} \mathbf{t}, \mathbf{k}=\mathbf{1}$, obtémse o operador de Laplace em coordenadas polares, que é dado por:

$$
\nabla^{2}=\frac{\partial^{2}}{\partial \mathbf{X}_{\mathrm{K}} \partial \mathbf{X}_{\mathrm{K}}}=\frac{\mathbf{d}^{2}}{\mathbf{d r}^{2}}+\frac{1}{\mathbf{r}} \frac{\mathbf{d}}{\mathbf{d r}}
$$


Logo, a equação diferencial em coordenadas polares é dada por:

$$
\nabla^{2} \nabla^{2} w=\frac{d^{4} w}{d r^{4}}+\frac{2}{r} \frac{d^{3} w}{d r^{3}}-\frac{1}{r^{2}} \frac{d^{2} w}{d r^{2}}+\frac{1}{r^{3}} \frac{d w}{d r}=\frac{g}{D}
$$

Utilizando-se os operadores (2.34) e (2.35) na equação (2.9), obtém-se os momentos:

$$
M_{i j}=-D\left\{\frac{d^{2} \mathbf{w}}{d r^{2}}\left[\delta_{i j} v+(1-v)\left(r,{ }_{i},,_{j}\right)\right]+\frac{1}{r} \frac{\mathbf{d w}}{\mathbf{d r}}\left[\delta_{\mathrm{ij}} v+(1-v)\left(\mathbf{t}_{\mathbf{i}} \mathbf{t}_{\mathbf{j}}\right)\right]\right\}
$$

Através dos operadores (2.30) e (2.35), obtém-se a derivada de terceira ordem, que é dada por:

$$
\mathbf{w},{ }_{k k i}=r,,_{i}\left(\frac{d^{3} w}{d r^{3}}+\frac{1}{r} \frac{d^{2} w}{d r^{2}}-\frac{1}{r^{2}} \frac{d w}{d r}\right)
$$

Substituindo-se (2.38) em (2.13), obtém-se o esforço cortante:

$$
Q_{j}=-D r,{ }_{j}\left(\frac{d^{3} \mathbf{w}}{\mathbf{d r}^{3}}+\frac{1}{\mathbf{r}} \frac{\mathbf{d}^{2} \mathbf{w}}{\mathbf{d r ^ { 2 }}}-\frac{1}{\mathbf{r}^{2}} \frac{\mathbf{d w}}{\mathbf{d r}}\right) \quad(\mathrm{j}=1,2)
$$

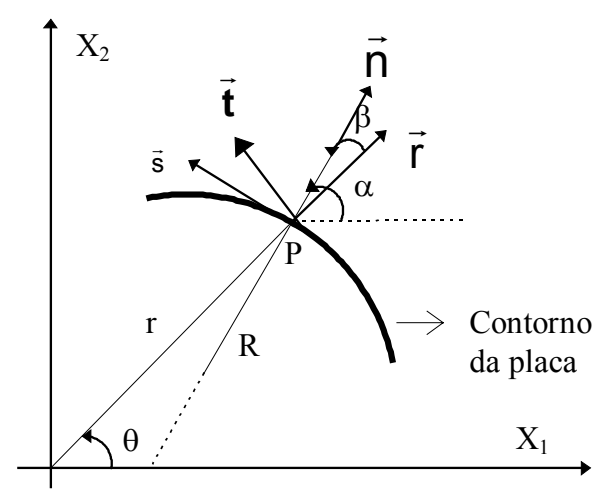

FIGURA 2.10 - Vetores n e s no Ponto P do Contorno da Placa

onde: $\quad \mathrm{n}_{1}=\cos \alpha, \mathrm{n}_{2}=\operatorname{sen} \alpha$;

$\mathrm{s}_{1}=-\operatorname{sen} \alpha, \mathrm{s}_{2}=\cos \alpha$

$\mathbf{R}$ é o raio da curvatura do contorno no ponto $\mathbf{P}$;

$$
\mathbf{r}, \mathbf{n}_{\mathrm{i}}=\cos \beta
$$




$$
\mathbf{r}_{i} \mathbf{s}_{i}=\cos \left(\beta+\frac{\pi}{2}\right)=-\operatorname{sen} \beta
$$

A fim de deduzir as expressões de $\mathbf{M}_{\mathbf{n}}, \mathbf{M}_{\mathbf{n}}$ e $\mathbf{V}_{\mathbf{n}}$ em coordenadas polares, considere um ponto $\mathbf{P}$ do contorno, onde $\mathbf{n}$ e $\mathbf{s}$ são, respectivamente, os vetores normal e tangente ao contorno, com origem em P, como mostra a figura (2.10). Substituindo-se (2.37) em (2.17) e (2.18), assim como (2.39) em (2.19), obtêm-se as equações:

$$
\begin{aligned}
& M_{n n}=-D\left\{\frac{d^{2} w}{d r^{2}}\left[v+(1-v)\left(r, n_{i} n_{i}\right)^{2}\right]+\frac{1}{r} \frac{d w}{d r}\left[v+(1-v)\left(r,,_{i} s_{i}\right)^{2}\right]\right\} \\
& M_{n s}=-D(1-v)\left(r, n_{i}\right)\left(r, s_{j}\right)\left(\frac{d^{2} w}{d r^{2}}-\frac{1}{r} \frac{d w}{d r}\right) \\
& Q_{n}=-D\left(r, n_{i}\right)\left(\frac{d^{3} w}{d r^{3}}+\frac{1}{r} \frac{d^{2} w}{d^{2}}-\frac{1}{r^{2}} \frac{d w}{d r}\right)
\end{aligned}
$$

Substituindo-se (2.40) e (2.41) em (2.43), obtém-se um nova expressão para $\mathbf{M}_{\mathbf{n s}}$ :

$$
M_{n s}=D(1-v) \cos \beta \operatorname{sen} \beta\left(\frac{d^{2} w}{d r^{2}}-\frac{1}{r} \frac{d w}{d r}\right)
$$

Para a obtenção de $\mathbf{V}_{\mathbf{n}}$, deve-se ter a expressão da derivada de $\mathbf{M}_{\mathbf{n}}$ em relação à direção s, que é dada por:

$$
\begin{gathered}
\frac{\partial \mathbf{M}_{\mathrm{ns}}}{\partial \mathbf{s}}=\frac{\partial \mathbf{M}_{\mathrm{ns}}}{\partial \beta} \frac{\partial \beta}{\partial \mathbf{s}}+\frac{\partial \mathbf{M}_{\mathrm{ns}}}{\partial \mathbf{r}} \frac{\partial \mathbf{r}}{\partial \mathbf{s}} \\
\text { onde }: \frac{\partial \mathbf{r}}{\partial \mathbf{s}}=\frac{\partial \mathbf{r}}{\partial \mathbf{x}_{1}} \frac{\partial \mathbf{x}_{1}}{\partial \mathbf{s}}+\frac{\partial \mathbf{r}}{\partial \mathbf{x}_{2}} \frac{\partial \mathbf{x}_{2}}{\partial \mathbf{s}}=\mathbf{r},{ }_{i} \mathbf{s}_{\mathbf{i}}=-\operatorname{sen} \beta \\
\frac{\partial \beta}{\partial \mathbf{s}}=\frac{\partial \alpha}{\partial \mathbf{s}}-\frac{\partial \theta}{\partial \mathbf{s}}=\left(\alpha, \mathbf{s}_{1}+\alpha, \mathbf{s}_{2}\right)-\left(\theta, \mathbf{s}_{1}+\theta,{ }_{2} \mathbf{s}_{2}\right)=\frac{1}{\mathbf{R}}-\frac{\cos \beta}{\mathbf{r}}
\end{gathered}
$$


Assim, derivando-se a equação (2.45) em relação a $\beta$ e $\mathbf{r}$, obtêm-se os outros dois termos de (2.46), a qual resulta em:

$$
\begin{aligned}
& \frac{\partial M_{n s}}{\partial s}=D(1-v) \cos \beta\left[\operatorname{sen}^{2} \beta\left(-\frac{d^{3} w}{d r^{3}}-\frac{1}{r} \frac{d^{2} w}{d r^{2}}+\frac{1}{r^{2}} \frac{d w}{d r}\right)+\right. \\
& \left.+\left(\frac{4 \operatorname{sen}^{2}(\beta)-1}{r}\right)\left(\frac{d^{2} w}{d r^{2}}-\frac{1}{r} \frac{d w}{d r}\right)\right]+ \\
& +\frac{D(1-v)}{R}\left(1-2 \operatorname{sen}^{2} \beta\right)\left(\frac{d^{2} w}{d r^{2}}-\frac{1}{r} \frac{d w}{d r}\right)
\end{aligned}
$$

Levando-se (2.49) e (2.44) em (2.20), obtém-se a expressão de $\mathbf{V}_{\mathbf{n}}$ :

$$
\begin{aligned}
& V_{n}=-D(1-v)\left(n_{i} r_{i}\right)\left\{\left[\left(s_{j} r,_{j}\right)^{2}+\frac{1}{1-v}\right]\left(\frac{d^{3} w}{d r^{3}}+\frac{1}{r} \frac{d^{2} w}{d r^{2}}-\frac{1}{r^{2}} \frac{d w}{d r}\right)+\right. \\
& \left.+\frac{1-4\left(s_{j} r,,_{j}\right)^{2}}{r}\left(\frac{d^{2} w}{d r^{2}}-\frac{1}{r} \frac{d w}{d r}\right)\right\} \\
& +\frac{D(1-v)}{R}\left[1-2\left(s_{i} r_{i}\right)^{2}\right]\left(\frac{d^{2} \mathbf{w}}{d r^{2}}-\frac{1}{r} \frac{d w}{d r}\right)
\end{aligned}
$$




\section{EQUAÇÕES INTEGRAIS PARA FLEXÃO DE PLACAS SUJEITAS À CARREGAMENTOS TRANSVERSAIS}

\subsection{Introdução}

Para a aplicação do Método dos Elementos de Contorno, é necessário, inicialmente, a obtenção de equações integrais que definam o problema, que podem ser obtidas através do método dos resíduos ponderados, ou a partir do Teorema da Reciprocidade de Betti. Neste trabalho, tais equações serão obtidas a partir do primeiro Teorema de Betti, onde a integração da expressão levará à equação integral de deslocamentos para pontos internos, e então a partir dessa obter-se-á a equação integral para pontos do contorno.

\subsection{Equação Integral para um Ponto do Domínio da Placa}

O Teorema de BETTI (1872) relaciona dois estados distintos de tensão e deformação existentes num sólido (placa) de domínio finito, causados por dois carregamentos não simultâneos. O mesmo é dado pela seguinte expressão:

$$
\int_{\mathbf{v}} \sigma_{\mathrm{ij}}^{1} \varepsilon_{\mathrm{ij}}^{2} \mathbf{d v}=\int_{\mathbf{v}} \sigma_{\mathrm{ij}}^{2} \varepsilon_{\mathrm{ij}}^{1} \mathbf{d v} \quad(\mathrm{i}, \mathrm{j},=1,2,3)
$$

Assim, escolhendo-se como estado 1 aquele relacionado ao problema fundamental, ainda a ser obtido, e como estado 2 aquele relacionado ao problema real, pode-se escrever o teorema da seguinte maneira: 


$$
\int_{\mathbf{v}} \sigma_{\mathrm{ij}}^{*} \varepsilon_{\mathrm{ij}} \mathbf{d v}=\int_{\mathbf{v}} \sigma_{\mathrm{ij}} \varepsilon_{\mathrm{ij}}^{*} \mathbf{d v} \quad(\mathrm{i}, \mathrm{j},=1,2,3)
$$

Denotando-se por $\mathbf{U}$ o segundo membro da equação (3.2) e considerando-se o estado plano de tensão, têm-se:

$$
\mathrm{U}=\int_{\mathrm{v}}\left(\sigma_{11} \varepsilon_{11}^{*}+\sigma_{22} \varepsilon_{22}^{*}+2 \sigma_{12} \varepsilon_{12}^{*}\right) \mathbf{d v}
$$

Considerando-se a equação (2.4) e fazendo-se a integração das tensões ao longo da espessura, a expressão de $\mathbf{U}$ pode ser escrita em função de uma integral sobre o domínio da seguinte forma:

$$
U=\int_{\Omega} \int_{-\frac{\mathrm{t}}{2}}^{\frac{\mathrm{t}}{2}}\left(\sigma_{\mathrm{ij}} \varepsilon_{\mathrm{ij}}^{*}\right) \mathbf{d} \mathbf{x}_{3} \mathbf{d} \Omega=-\int_{\Omega} \int_{-\mathrm{t} / 2}^{\mathrm{t} / 2}\left(\sigma_{\mathrm{ij}} \mathbf{x}_{3} \mathbf{w}_{\mathrm{ij}}^{*}\right) \mathbf{d} \mathbf{x}_{3} \mathbf{d} \Omega=-\int_{\Omega}\left(\mathbf{M}_{\mathrm{ij}} \mathbf{w}, \stackrel{*}{\mathrm{ij}}\right) \mathbf{d} \Omega \quad(\mathrm{i}, \mathrm{j}=1,2)
$$

Desse modo, considere uma placa isótropa qualquer de contorno $\Gamma$ e domínio $\Omega$, a qual está contida em outra, de domínio infinito $\Omega_{\infty}$ e contorno $\Gamma_{\infty}$ conforme a figura (3.1).

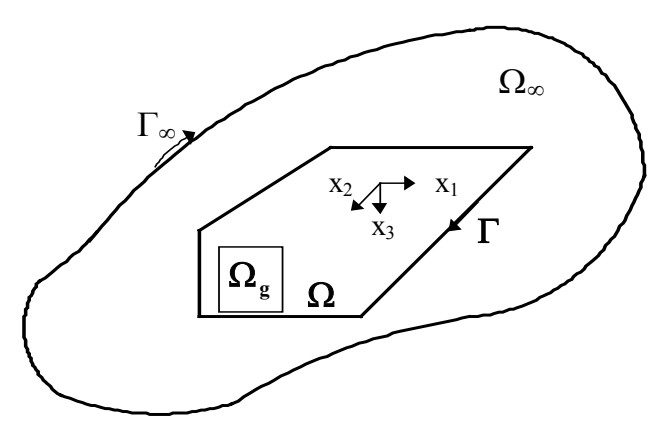

FIGURA 3.1 - Placa de Dimensões Finitas, Contida em uma Placa Infinita

Define-se como problema fundamental, o caso de uma carga transversal unitária $\mathbf{g}^{\text {* }}$ aplicada em um ponto genérico $\mathbf{q}$ do domínio infinito $\Omega_{\infty}$, denominado domínio fundamental, que provocará, em um ponto $\mathbf{p}$ qualquer da mesma, um deslocamento transversal $\mathbf{w}^{*}$, um estado de tensão $\sigma_{\mathrm{ij}}^{*}$ e um estado de deformação $\varepsilon_{\mathrm{ij}}^{*}$. O ponto $\mathbf{q}$ é denominado ponto de 
carregamento ou ponto fonte e o ponto $\mathbf{p}$ ponto de deslocamento ou ponto campo. O centro do sistema de coordenadas polares coincide com o ponto q, como é mostrado na figura (3.2).

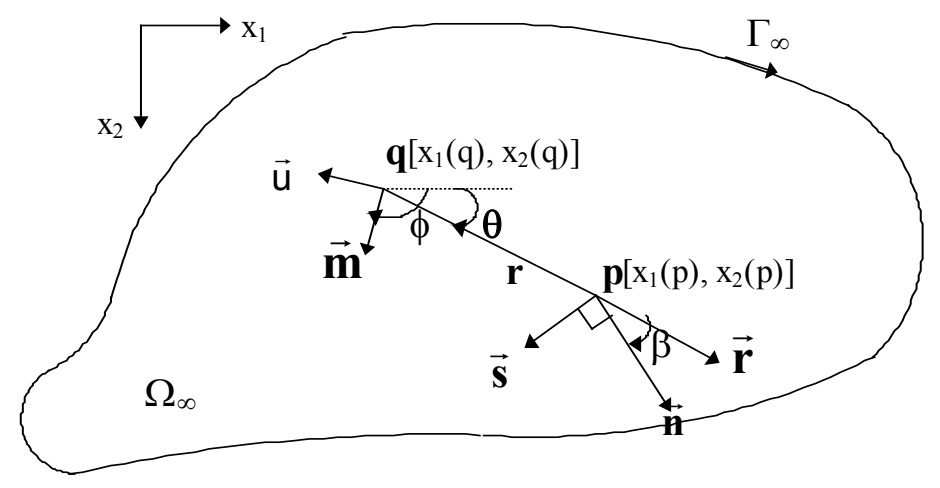

FIGURA 3.2 - Pontos de Carregamento q e de Deslocamento p e Sistemas de Coordenadas $(\mathrm{m}, \mathrm{u})$ e $(\mathrm{n}, \mathrm{s})$ no Domínio de uma Placa Infinita.

$$
\text { onde } \mathbf{r}=\sqrt{\left[\mathbf{x}_{1}(\mathbf{p})-\mathbf{x}_{1}(\mathbf{q})\right]^{2}+\left[\mathbf{x}_{2}(\mathbf{p})-\mathbf{x}_{2}(\mathbf{q})\right]^{2}}
$$

é a distância entre os pontos $\mathbf{q}$ e $\mathbf{p}$.

A carga $\mathbf{g}^{*}$ é definida através da distribuição delta de Dirac, denotada por $\delta(\mathbf{q}, \mathbf{p})$, cujas propriedades são:

$$
\begin{aligned}
& \mathbf{g}^{*}=\delta(\mathbf{q}, \mathbf{p})=\left\{\begin{array}{lll}
\mathbf{0} & \text { para } & \mathbf{p} \neq \mathbf{q} \\
\infty & \text { para } & \mathbf{p} \equiv \mathbf{q}
\end{array}\right. \\
& \int_{\Omega_{\infty}} \phi(\mathbf{p}) \delta(\mathbf{q}, \mathbf{p}) \mathbf{d} \Omega_{\infty}=\phi(\mathbf{q})
\end{aligned}
$$

sendo $\Phi$ uma função contínua qualquer. Observando-se as propriedades, dadas pelas equações (3.6) e (3.7), conclui-se que a resultante do carregamento definido por $\delta(\mathbf{q}, \mathbf{p})$ sobre o domínio fundamental é uma força unitária aplicada no ponto $\mathbf{q}$

$$
\int_{\Omega_{\infty}} \delta(\mathbf{q}, \mathbf{p}) \mathbf{d} \Omega_{\infty}=\mathbf{1}
$$


O problema real é aquele relativo a um carregamento g qualquer distribuído em uma área de domínio $\Omega_{\mathrm{g}}$, contida no domínio $\Omega$ da placa finita. Do mesmo modo, o carregamento g provocará em $\mathbf{p}$ um deslocamento transversal $\mathbf{w}$, um estado de tensão $\sigma_{\mathrm{ij}}$ e um estado de deformação $\varepsilon_{\mathrm{ij}}$.

Os deslocamentos e esforços relativos ao problema fundamental são funções do ponto $\mathbf{q}$ de aplicação da carga e do ponto $\mathbf{p}$ de deslocamento do domínio; se esse último estiver no contorno da placa, é representado por $\mathbf{P}$. Já aqueles relativos ao problema real são funções apenas do ponto $\mathbf{p}$, pois a posição deste carregamento é fixa.

Aplicando-se o teorema da divergência, ou integrando-se (3.4) por partes, em relação à coordenada $\mathbf{x}_{\mathbf{j}}$, obtém-se:

$$
\mathbf{U}=-\int_{\Gamma}\left(\mathbf{M}_{\mathrm{ij}} \mathbf{w}_{\mathrm{i}}^{*} \mathbf{n}_{\mathrm{j}}\right) \mathrm{d} \Gamma+\int_{\Omega}\left(\mathbf{M}_{\mathrm{ij}},{ }_{\mathrm{j}} \mathbf{w},{ }_{\mathrm{i}}^{*}\right) \mathrm{d} \Omega
$$

onde $\mathbf{n}_{\mathbf{j}}$ são os cossenos diretores do versor normal ao contorno da placa.

Integrando-se (3.9) novamente por partes, obtém-se:

$$
\mathbf{U}=-\int_{\Gamma}\left(\mathbf{M}_{\mathrm{ij}} \mathbf{w}_{\mathrm{i}}^{*} \mathbf{n}_{\mathrm{j}}\right) \mathrm{d} \Gamma+\int_{\Gamma}\left(\mathbf{M}_{\mathrm{ij}},{ }_{\mathrm{j}} \mathbf{w}^{*} \mathbf{n}_{\mathrm{i}}\right) \mathrm{d} \Gamma-\int_{\Omega}\left(\mathbf{M}_{\mathrm{i}, \mathrm{i}},_{\mathrm{ij}} \mathbf{w}^{*}\right) \mathrm{d} \Omega
$$

Considerando-se as equações (2.13), (2.14) e (2.19), a expressão de $\mathbf{U}$ resulta em:

$$
\mathbf{U}=-\int_{\Gamma}\left(M_{\mathrm{ij}} \mathbf{w}_{\mathrm{i}}^{*} \mathbf{n}_{\mathrm{j}}\right) \mathrm{d} \Gamma+\int_{\Gamma}\left(\mathbf{Q}_{\mathbf{n}} \mathbf{w}^{*}\right) \mathbf{d} \Gamma+\int_{\Omega}\left(\mathbf{g w}^{*}\right) \mathrm{d} \Omega
$$

onde: $\quad \mathbf{w}^{*},_{\mathbf{i}}=\frac{\partial \mathbf{w}^{*}}{\partial \mathbf{x}_{\mathbf{i}}}=\frac{\partial \mathbf{w}^{*}}{\partial \mathbf{n}} \frac{\partial \mathbf{n}}{\partial \mathbf{x}_{\mathbf{i}}}+\frac{\partial \mathbf{w}^{*}}{\partial \mathbf{s}} \frac{\partial \mathbf{s}}{\partial \mathbf{x}_{\mathbf{i}}}=\frac{\partial \mathbf{w}^{*}}{\partial \mathbf{n}} \mathbf{n}_{\mathbf{i}}+\frac{\partial \mathbf{w}^{*}}{\partial \mathbf{s}} \mathbf{s}_{\mathbf{i}}$

$\mathbf{s}_{\mathbf{i}}$ são os cossenos diretores do versor tangente ao contorno.

Substituindo-se (3.12) em (3.11), tem-se:

$$
\mathbf{U}=-\int_{\Gamma}\left(\mathbf{M}_{\mathrm{ij}} \mathbf{n}_{\mathrm{i}} \mathbf{n}_{\mathrm{j}} \frac{\partial \mathbf{w}^{*}}{\partial \mathbf{n}}+\mathbf{M}_{\mathrm{ij}} \mathbf{s}_{\mathrm{i}} \mathbf{n}_{\mathrm{j}} \frac{\partial \mathbf{w}^{*}}{\partial \mathbf{s}}\right) \mathbf{d} \Gamma+\int_{\Gamma}\left(\mathbf{Q}_{\mathbf{n}} \mathbf{w}^{*}\right) \mathbf{d} \Gamma+\int_{\Omega}\left(\mathbf{g w}^{*}\right) \mathbf{d} \Omega
$$


Considerando-se as equações (2.17) e (2.18), pode-se escrever:

$$
\begin{aligned}
& \mathbf{U}=-\int_{\Gamma}\left(\mathbf{M}_{\mathbf{n n}} \frac{\partial \mathbf{w}^{*}}{\partial \mathbf{n}}+\mathbf{M}_{\mathbf{n s}} \frac{\partial \mathbf{w}^{*}}{\partial \mathbf{s}}-\mathbf{Q}_{\mathbf{n}} \mathbf{w}^{*}\right) \mathbf{d} \Gamma+\int_{\Omega}\left(\mathbf{g w}^{*}\right) \mathbf{d} \Omega \\
& \text { Integrando-se a parcela } \int_{\Gamma}\left(\mathbf{M}_{\mathrm{ns}} \frac{\partial \mathbf{w}^{*}}{\partial \mathbf{s}}\right) \mathbf{d} \Gamma \text { por partes, obtém-se: } \\
& \int_{\Gamma}\left(\mathbf{M}_{\mathbf{n s}} \frac{\partial \mathbf{w}^{*}}{\partial \mathbf{s}}\right) \mathbf{d} \Gamma=\left[\mathbf{M}_{\mathrm{ns}} \mathbf{w}^{*}\right]_{\Gamma_{1}}^{\Gamma_{2}}-\int_{\Gamma} \frac{\partial \mathbf{M}_{\mathbf{n s}}}{\partial \mathbf{s}} \mathbf{w}^{*} \mathbf{d} \Gamma
\end{aligned}
$$

onde $\Gamma_{1}$ e $\Gamma_{2}$ representam os limites do contorno no qual se realiza a integração.

Para contornos fechados, onde a representação paramétrica de $\mathbf{M}_{\mathbf{n}}$ e a respectiva derivada forem contínuas, a primeira parcela do segundo membro da equação (3.15) se anula, mas quando existirem angulosidades ou cantos no contorno, esta parcela não se anula, dando origem à reação de canto $\mathbf{R}_{\mathbf{c i}}$ (ver item 2.2.7). Assim, considerando-se (2.21), a equação (3.15) pode ser expressa por:

$$
\int_{\Gamma}\left(\mathbf{M}_{\mathrm{ns}} \frac{\partial \mathbf{w}^{*}}{\partial \mathbf{s}}\right) \mathbf{d} \Gamma=-\sum_{\mathrm{i}=1}^{\mathbf{N}_{\mathrm{c}}} \mathbf{R}_{\mathrm{ci}} \mathbf{w}_{\mathrm{ci}}^{*}-\int_{\Gamma} \frac{\partial \mathbf{M}_{\mathrm{ns}}}{\partial \mathbf{s}} \mathbf{w}^{*} \mathbf{d} \Gamma
$$

onde $\mathbf{N}_{\mathbf{c}}$ é o número total de cantos e $\mathbf{w}_{\mathbf{c i}}^{*}$ é o valor do deslocamento $\mathbf{w}^{*}$ no canto $\mathbf{i}$.

Substituindo-se (3.16) em (3.14), considerando-se (2.20) e também o fato que g está distribuída em $\Omega_{\mathrm{g}}$, chega-se à:

$$
\mathbf{U}=\int_{\Gamma}\left(\mathbf{V}_{\mathbf{n}} \mathbf{w}^{*}-\mathbf{M}_{\mathrm{nn}} \frac{\partial \mathbf{w}^{*}}{\partial \mathbf{n}}\right) \mathbf{d} \Gamma+\sum_{\mathrm{i}=1}^{\mathbf{N}_{\mathrm{c}}} \mathbf{R}_{\mathrm{ci}} \mathbf{W}_{\mathrm{ci}}^{*}+\int_{\Omega_{\mathrm{g}}}\left(\mathbf{g w}^{*}\right) \mathbf{d} \Omega_{\mathrm{g}}
$$

Analogamente, obtém-se o primeiro termo de (3.2). Considerando-se a propriedade da função delta de Dirac, dada por (3.7), tem-se que:

$$
\int_{\Omega}\left(\mathbf{g}^{*} \mathbf{w}\right) \mathbf{d} \Omega=\int_{\Omega} \delta(\mathbf{q}, \mathbf{p}) \mathbf{w}(\mathbf{p}) \mathbf{d} \Omega=\mathbf{w}(\mathbf{q})
$$


onde $\mathbf{w}(\mathbf{q})$ é o deslocamento devido à carga $\mathbf{g}$.

Desse modo, obtém-se a equação integral do deslocamento de um ponto $\mathbf{q}$ do domínio da placa, em função dos deslocamentos e esforços do contorno, que é dada por:

$$
\begin{aligned}
& \mathbf{w}(\mathbf{q})+\int_{\Gamma}\left(\mathbf{V}_{\mathbf{n}}^{*}(\mathbf{q}, \mathbf{P}) \mathbf{w}(\mathbf{P})-\mathbf{M}_{\mathrm{nn}}^{*}(\mathbf{q}, \mathbf{P}) \frac{\partial \mathbf{w}}{\partial \mathbf{n}}(\mathbf{P})\right) \mathbf{d} \Gamma(\mathbf{P})+\sum_{\mathrm{i}=1}^{\mathbf{N}_{\mathrm{c}}} \mathbf{R}_{\mathrm{ci}}^{*}(\mathbf{q}, \mathbf{P}) \mathbf{w}_{\mathrm{ci}}(\mathbf{P})= \\
& =\int_{\Gamma}\left(\mathbf{V}_{\mathrm{n}}(\mathbf{P}) \mathbf{w}^{*}(\mathbf{q}, \mathbf{P})-\mathbf{M}_{\mathrm{nn}}(\mathbf{P}) \frac{\partial \mathbf{w}^{*}}{\partial \mathbf{n}}(\mathbf{q}, \mathbf{P})\right) \mathbf{d} \Gamma(\mathbf{P})+\sum_{\mathrm{i}=\mathbf{1}}^{\mathbf{N}_{\mathrm{c}}} \mathbf{R}_{\mathrm{ci}}(\mathbf{P}) \mathbf{w}_{\mathrm{ci}}^{*}(\mathbf{q}, \mathbf{P})+ \\
& \quad+\int_{\Omega_{\mathrm{g}}}\left(\mathbf{g}(\mathbf{p}) \mathbf{w}^{*}(\mathbf{q}, \mathbf{p})\right) \mathbf{d} \Omega_{\mathrm{g}}(\mathbf{p})
\end{aligned}
$$

Derivando-se (3.19) em relação a $\mathbf{m}$, obtém-se a equação integral da derivada direcional do deslocamento em relação à direção m, como é mostrado no trabalho de PAIVA (1987).

\subsection{1 - Solução Fundamental de Placas}

A solução fundamental corresponde ao deslocamento $\mathbf{w}^{*}$ de um ponto $\mathbf{p}$, que é causado por uma carga unitária transversal aplicada em q. É obtida, substituindo-se g pela distribuição delta de Dirac em (2.36), isto é:

$$
\nabla^{2} \nabla^{2} \mathbf{w}^{*}=\frac{\mathbf{d}^{4} \mathbf{w}^{*}}{\mathbf{d r} \mathbf{r}^{4}}+\frac{2}{\mathbf{r}} \frac{\mathbf{d}^{3} \mathbf{w}^{*}}{\mathbf{d r}^{3}}-\frac{1}{\mathbf{r}^{2}} \frac{\mathbf{d}^{2} \mathbf{w}^{*}}{\mathbf{d r}^{2}}+\frac{1}{\mathbf{r}^{3}} \frac{\mathbf{d w} *}{\mathbf{d r}}=\frac{\delta(\mathbf{q}, \mathbf{p})}{\mathrm{D}}
$$

Considerando-se a equação (3.6), pode-se dizer que esta última é nula para todos os pontos do domínio fundamental, com exceção do ponto q. Assim, $\mathbf{w}^{*}$ deve ser tal que satisfaça a equação $\nabla^{2} \nabla^{2} \mathbf{w}^{*}=\mathbf{0}$. Fazendo-se integrações sucessivas, obtém-se:

$$
w^{*}=\frac{C_{1}}{4} r^{2} \ln r+\left(C_{2}-C_{1}\right) \frac{r^{2}}{8}+C_{3} \ln r+C_{4}
$$

A constante $\mathbf{C}_{3}=\mathbf{0}$ é obtida escrevendo-se a condição de simetria em relação ao ponto $\mathbf{q}$ (figura 3.3), isto é, $\mathbf{d w} * / \mathbf{d r}=\mathbf{0}$, para $\mathbf{r}=\mathbf{0}$. 
$\underset{\mathbf{x}_{2}}{\longrightarrow} \mathbf{x}_{1}$

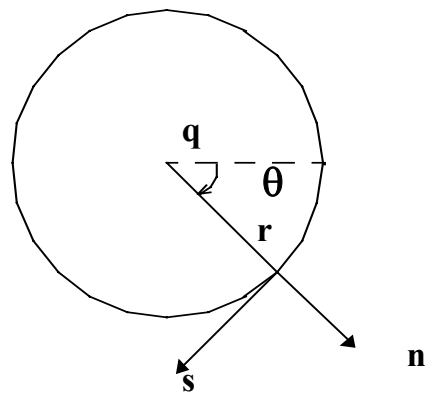

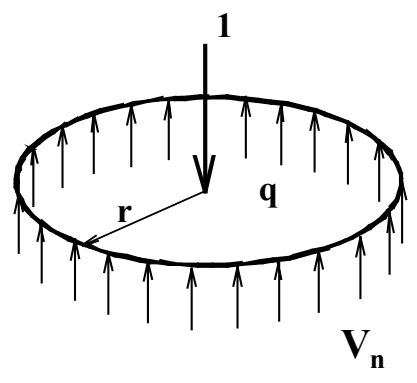

FIGURA 3.3 - Forças Atuantes no Círculo de Raio r e Centro q.

Fazendo-se o equilíbrio das forças verticais atuantes em um círculo de raio $\mathbf{r}$ e centro q (ver figura 3.3), sendo q o ponto de aplicação da carga unitária, obtém-se que a força equivalente $\mathbf{V}_{\mathbf{n}}$ em um ponto $\mathbf{p}$ qualquer da circunferência, necessária para equilibrar a carga unitária, vale:

$$
V_{n}=-\frac{1}{2 \pi r}
$$

Nesse caso, a expressão de $\mathbf{V}_{\mathbf{n}}$, dada em (2.50), é função apenas de $\mathbf{r}$, pois o ângulo $\beta$, definido na figura (2.10), é nulo para todos os pontos da circunferência. Logo, tem-se:

$$
V_{n}=-D\left(\frac{d^{3} w}{d r^{3}}+\frac{1}{r} \frac{d^{2} w^{*}}{d r^{2}}-\frac{1}{r^{2}} \frac{d w^{*}}{d r}\right)
$$

Substituindo-se (3.23) em (3.22) e expressando-se $\mathbf{V}_{\mathbf{n}}$ em função do Laplaciano em coordenadas polares, tem-se:

$$
-D \frac{d}{d r}\left(\frac{d^{2} w^{*}}{d r^{2}}+\frac{1}{r} \frac{d w^{*}}{d r}\right)=-\frac{1}{2 \pi r}
$$

Das equações (3.24) e (3.21), obtém-se: $\mathbf{C}_{\mathbf{1}}=\mathbf{1} / \mathbf{2} \pi \mathbf{D}$. As constantes $\mathbf{C}_{\mathbf{2}}$ e $\mathbf{C}_{\mathbf{4}}$ são obtidas a partir das condições de contorno de um problema qualquer de placa, mas como no problema fundamental o raio é infinito, estes valores poderiam ser correspondentes a 
quaisquer deslocamentos de corpo rígido. Nesse trabalho será adotada a sugestão de DANSON (1979), que também foi adotada por CHUEIRI (1994), que considera $\mathbf{C}_{\mathbf{2}}=\mathbf{C}_{\mathbf{4}}=\mathbf{0}$. Logo, substituindo-se os valores das constantes em (3.21), obtém-se a expressão da solução fundamental:

$$
\mathbf{w}^{*}=\frac{1}{8 \pi \mathbf{D}} \mathbf{r}^{2}\left(\ln \mathbf{r}-\frac{1}{2}\right)
$$

Para obter-se a expressão da rotação fundamental para um ponto $\mathbf{p}$, basta derivar a expressão (3.25) em relação a n, ou seja:

$$
\frac{\partial \mathbf{w}^{*}}{\partial \mathbf{n}}=\frac{\mathbf{d w ^ { * }}}{\mathbf{d r}} \frac{\partial \mathbf{r}}{\partial \mathbf{n}}=\frac{\mathbf{d w ^ { * }}}{\mathbf{d r}}\left(\frac{\partial \mathbf{r}}{\partial \mathbf{x}_{\mathbf{i}}(\mathbf{p})} \frac{\partial \mathbf{x}_{\mathbf{i}}(\mathbf{p})}{\partial \mathbf{n}}\right)=\frac{\mathbf{d w ^ { * }}}{\mathbf{d r}} \mathbf{n}_{\mathbf{i}} \mathbf{r}_{\mathbf{i}}
$$

resultando em:

$$
\frac{\partial w^{*}}{\partial \mathbf{n}}=\frac{\mathbf{r}}{4 \pi D} \ln r\left(\mathbf{r},_{i} n_{i}\right)
$$

A partir da expressão de $\mathbf{r}$, dada pela equação (3.5), obtêm-se:

$$
\begin{aligned}
& \frac{\partial \mathbf{r}}{\partial \mathbf{x}_{\mathrm{i}}(\mathbf{p})}=\mathbf{r},_{\mathrm{i}}=\frac{\mathbf{x}_{\mathrm{i}}(\mathbf{p})-\mathbf{x}_{\mathrm{i}}(\mathbf{q})}{\mathbf{r}} \\
& \frac{\partial^{2} \mathbf{r}}{\partial \mathbf{x}_{\mathrm{i}}(\mathbf{p}) \partial \mathbf{x}_{\mathrm{j}}(\mathbf{p})}=\mathbf{r},_{\mathrm{ij}}=\frac{\mathbf{r},,_{\mathrm{i}} \cdot \mathbf{r},_{\mathrm{j}}-\delta_{\mathrm{ij}}}{\mathbf{r}}
\end{aligned}
$$

onde $\delta_{\mathrm{ij}}$ é o delta de Kronecker.

Logo, derivando-se (3.25), e a partir de (2.42), (2.43) e (2.50), pode-se obter as expressões dos esforços do problema fundamental, segundo um sistema de coordenadas (n, s) qualquer (Figura 3.2), que são dadas por:

$$
M_{n}^{*}=-\frac{1}{4 \pi}\left[(1+v) \ln r+(1-v)\left(r, n_{i}\right)^{2}+v\right]
$$




$$
\begin{aligned}
& M_{n s}^{*}=-\frac{1}{4 \pi}(1-v)\left(r, n_{i} n_{i}\right)\left(r, s_{j} s_{j}\right) \\
& V_{n}^{*}=\frac{r, n_{i} n_{i}}{4 \pi r}\left[2(1-v)\left(r, s_{j} s_{j}\right)^{2}-3+v\right]+\frac{1-v}{4 \pi R}\left[1-2\left(r, s_{i} s_{i}\right)^{2}\right]
\end{aligned}
$$

onde $\mathbf{r}$, e $\mathbf{r}_{\mathbf{i}}$ são dados, respectivamente, por (3.5) e (2.25) e $\mathbf{n}_{\mathbf{i}}, \mathbf{s}_{\mathbf{i}}$ estão definidos na figura (2.10).

Para o caso em que o contorno da placa é aproximado por elementos retos, a curvatura $\mathbf{R}$ em qualquer ponto do contorno tende ao infinito. Nesse caso, a expressão de $\mathbf{V}_{\mathbf{n}}^{*}$ passa a ser:

$$
V_{n}^{*}=\frac{r, n_{i}}{4 \pi r}\left[2(1-v)\left(r, s_{j} s_{j}\right)^{2}-3+v\right]
$$

De acordo com o item (2.2.7), a reação de canto, referente ao problema fundamental, é dada por:

$$
\mathbf{R}_{\mathrm{ci}}^{*}=\mathbf{M}_{\mathrm{nsi}}^{*(+)}-\mathbf{M}_{\mathrm{nsi}}^{*(-)}
$$

Para a obtenção dos esforços nos pontos internos, como é mostrado no capítulo (4), necessita-se das expressões das derivadas segundas e terceiras dos deslocamentos e esforços fundamentais. Assim, derivando-se as expressões (3.25), (3.27), (3.30) e (3.32) e considerando a equação (3.29), obtêm-se:

$$
\begin{aligned}
& \frac{\partial w^{*}}{\partial x_{i}}(q, p)=-\frac{r \ln (r)}{4 \pi D} r_{, i} \\
& \frac{\partial^{2} w^{*}}{\partial x_{i} \partial x_{j}}(q, p)=\frac{1}{4 \pi D}\left(r, r_{i}, \delta_{i j} \ln (r)\right) \\
& \frac{\partial^{2} w^{*}}{\partial x_{k} \partial x_{k}}(q, p)=\frac{1}{4 \pi D}(1+2 \ln (r)) \\
& \frac{\partial}{\partial x_{i}}\left(\frac{\partial^{2} w^{*}}{\partial x_{k} \partial x_{k}}(q, p)\right)=\frac{-r,,_{i}}{2 \pi D r}
\end{aligned}
$$


35

$$
\begin{aligned}
& \frac{\partial}{\partial \mathbf{x}_{i}}\left(\frac{\partial w^{*}}{\partial \mathbf{n}}(\mathbf{q}, \mathbf{p})\right)=\frac{-1}{4 \pi D}\left[\mathbf{r},{ }_{i}\left(\mathbf{r}, n_{k}\right)+\ln (r) n_{i}\right] \\
& \frac{\partial^{2}}{\partial \mathbf{x}_{i} \partial \mathbf{x}_{j}}\left(\frac{\partial w^{*}}{\partial n}(\mathbf{q}, \mathbf{p})\right)=\frac{1}{4 \pi D r}\left[\left(\delta_{i j}-2 r,,_{i},,_{j}\right) \cdot\left(r, n_{k}, n_{k}\right)+r, n_{j}+r, n_{i}\right] \\
& \frac{\partial^{2}}{\partial \mathbf{x}_{\mathbf{k}} \partial \mathbf{x}_{\mathbf{k}}}\left(\frac{\partial w^{*}}{\partial \mathbf{n}}(\mathbf{q}, \mathbf{p})\right)=\frac{\mathbf{r},_{\mathbf{k}} \mathbf{n}_{\mathbf{k}}}{2 \pi \mathbf{D r}} \\
& \frac{\partial}{\partial \mathbf{x}_{\mathbf{i}}}\left(\frac{\partial^{2}}{\partial \mathbf{x}_{\mathbf{k}} \partial \mathbf{x}_{\mathbf{k}}}\left(\frac{\partial w^{*}}{\partial \mathbf{n}}(\mathbf{q}, \mathbf{p})\right)\right)=\frac{1}{2 \pi D r^{2}}\left[2 \mathbf{r},{ }_{i}\left(\mathbf{r},{ }_{k}, n_{k}\right)-n_{i}\right] \\
& \frac{\partial \mathbf{M}_{\mathbf{n s}}^{*}}{\partial \mathbf{x}_{\mathbf{i}}}(\mathbf{q}, \mathbf{p})=\frac{(1-v)}{4 \pi \mathbf{r}}\left[\mathbf{n}_{\mathbf{i}}\left(\mathbf{r},{ }_{\ell} \mathbf{s}_{\ell}\right)+\mathbf{s}_{\mathbf{i}}\left(\mathbf{r}, \mathbf{k}_{\mathbf{k}} \mathbf{n}_{\mathbf{k}}\right)-2 \mathbf{r}, \mathbf{i}\left(\mathbf{r}, \mathbf{n}_{\mathbf{k}}\right)\left(\mathbf{r}, \mathbf{s}_{\ell}\right)\right]
\end{aligned}
$$

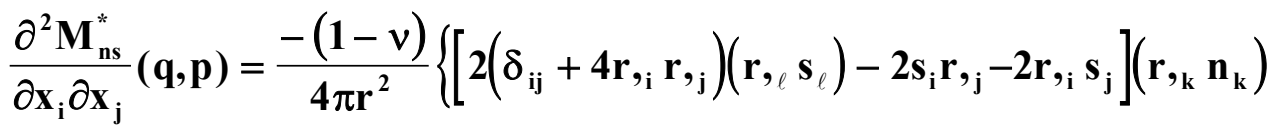

$$
\begin{aligned}
& \left.-2\left(r, n_{j}+r, n_{i}\right)\left(r, s_{\ell}\right) \cdot+n_{i} s_{j}+s_{i} n_{j}\right\} \\
& \frac{\partial^{2} \mathbf{M}_{\mathbf{n s}}^{*}}{\partial \mathbf{x}_{\mathbf{k}} \partial \mathbf{x}_{\mathbf{k}}}(\mathbf{q}, \mathbf{p})=\frac{(1-v)}{\pi \mathbf{r}^{2}}\left\{\left(\mathbf{s}_{\ell} \mathbf{r},{ }_{\ell}\right)\left(\mathbf{r}, \mathbf{n}_{\mathbf{k}}\right)\right\} \\
& \frac{\partial}{\partial \mathbf{x}_{\mathbf{i}}}\left(\frac{\partial^{2} \mathbf{M}_{\mathbf{n s}}^{*}}{\partial \mathbf{x}_{\mathbf{k}} \partial \mathbf{x}_{\mathbf{k}}}(\mathbf{q}, \mathbf{p})\right)=\frac{(1-v)}{\pi \mathbf{r}^{3}}\left[4 \mathbf{r},{ }_{i}\left(\mathbf{r},{ }_{k} s_{k}\right)\left(\mathbf{r},{ }_{\ell} \mathbf{n}_{\ell}\right)-\mathbf{n}_{\mathbf{i}}\left(\mathbf{r},{ }_{k} \mathbf{s}_{\mathbf{k}}\right)+\right. \\
& \left.-\mathbf{s}_{\mathbf{i}}\left(\mathbf{r}, \mathbf{n}_{\ell}\right)\right] \\
& \frac{\partial \mathbf{M}_{n}^{*}}{\partial x_{i}}(\mathbf{q}, \mathbf{p})=\frac{1}{4 \pi r}\left\{(1+v) r,,_{i}-2(1-v)\left(r, n_{k}\right)\left[r,\left(r, n_{k} n_{k}\right)-n_{i}\right]\right\} \\
& \frac{\partial^{2} \mathbf{M}_{n}^{*}}{\partial \mathbf{x}_{i} \partial \mathbf{x}_{j}}(\mathbf{q}, \mathbf{p})=-\frac{1}{4 \pi r^{2}}\left\{(1+v)\left(\delta_{i j}-2 r,{ }_{i},,_{j}\right)+2(1-v)\left[n_{i} n_{j}+\right.\right. \\
& -2 r,{ }_{j} n_{i}\left(r, n_{k}\right)-\left(\delta_{i j}-2 r, r_{j},,_{k}\right)\left(r, n_{k}\right)^{2}+ \\
& \left.\left.-2 \mathbf{r},_{i}\left(\mathbf{r}, n_{k}\right)\left[n_{j}-r,{ }_{j}\left(r, n_{k}\right)\right]\right]\right\} \\
& \frac{\partial^{2} \mathbf{M}_{n}^{*}}{\partial \mathbf{x}_{k} \partial \mathbf{x}_{k}}(\mathbf{q}, \mathbf{p})=-\frac{1}{4 \pi r^{2}}\left\{2(1-v)\left[1-2\left(r, n_{k} n^{2}\right]\right\}\right.
\end{aligned}
$$




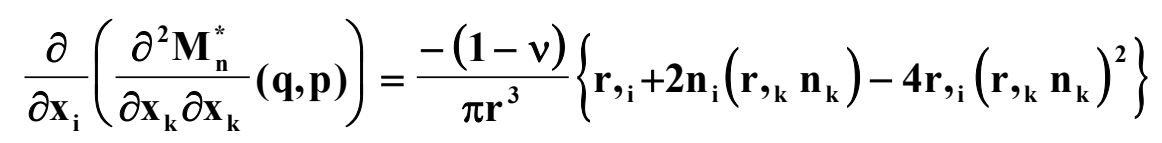

$$
\begin{aligned}
& \frac{\partial \mathbf{V}_{\mathbf{n}}^{*}}{\partial \mathbf{x}_{\mathbf{i}}}(\mathbf{q}, \mathbf{p})=\frac{1}{4 \pi \mathbf{r}^{2}}\left\{2(1-v)\left(\mathbf{r}, s_{\ell}\right)^{2}\left[4 \mathbf{r},{ }_{i}\left(\mathbf{r}, \mathbf{n}_{\mathbf{k}}\right)-\mathbf{n}_{\mathbf{i}}\right]+\right. \\
& \left.-4(1-v)\left(r, s_{\ell}\right)\left(r,{ }_{k} n_{k}\right) s_{i}+(3-v)\left[n_{i}-2 r,{ }_{i}\left(r, n_{k} n_{k}\right)\right]\right\} \\
& \frac{\partial^{2} \mathbf{V}_{\mathbf{n}}^{*}}{\partial \mathbf{x}_{\mathbf{i}} \partial \mathbf{x}_{\mathbf{j}}}(\mathbf{q}, \mathbf{p})=\frac{1}{4 \pi \mathbf{r}^{3}}\left\{2 ( 1 - v ) ( \mathbf { r } , { } _ { \ell } \mathbf { s } _ { \ell } ) ^ { 2 } \left[24 \mathbf{r},{ }_{\mathbf{i}},,_{\mathbf{j}}\left(\mathbf{r},{ }_{\mathbf{k}} \mathbf{n}_{\mathbf{k}}\right)+\right.\right. \\
& \left.-4\left[r,{ }_{i} n_{j}+r, n_{j} n_{i}+\delta_{i j}\left(r, n_{k}\right)\right]\right]+2(1-v)\left(r, s_{\ell}\right)\left[2\left(n_{i} s_{j}+n_{j} s_{i}\right)+\right. \\
& \left.-8\left(r,{ }_{k} n_{k}\right)\left(r, s_{j}+r, s_{i}\right)\right]+4(1-v)\left(r, n_{k} n_{k}\right)\left(s_{i} s_{j}\right)+ \\
& \left.+(3-v)\left[2 \delta_{i j}\left(r,{ }_{k} n_{k}\right)-8 r,{ }_{i},,_{j}\left(r,{ }_{k} n_{k}\right)+2\left(r, n_{i} n_{j}+r,{ }_{j} n_{i}\right)\right]\right\} \\
& \frac{\partial^{2} V_{n}^{*}}{\partial \mathbf{x}_{\mathrm{k}} \partial \mathbf{x}_{\mathrm{k}}}(\mathbf{q}, \mathbf{p})=-\frac{(1-v)}{4 \pi \mathbf{r}^{3}}\left(\mathbf{r}, \mathrm{n}_{\mathrm{k}} \mathbf{n}_{\mathrm{k}}\right)\left[4\left(\mathbf{r}, \mathrm{s}_{\ell}\right)^{2}-1\right] \\
& \frac{\partial}{\partial \mathbf{x}_{\mathbf{i}}}\left(\frac{\partial^{2} \mathbf{V}_{\mathbf{n}}^{*}}{\partial \mathbf{x}_{\mathbf{k}} \partial \mathbf{x}_{\mathbf{k}}}(\mathbf{q}, \mathbf{p})\right)=-\frac{(1-v)}{\pi \mathbf{r}^{4}}\left\{\left(\mathbf{r}, \mathbf{s}_{\ell}\right)^{2}\left[24 \mathbf{r}, \mathbf{i}_{\mathbf{i}}\left(\mathbf{r}, \mathbf{k}_{\mathbf{k}} \mathbf{n}_{\mathbf{k}}\right)-4 \mathbf{n}_{\mathbf{i}}\right]+\right.
\end{aligned}
$$

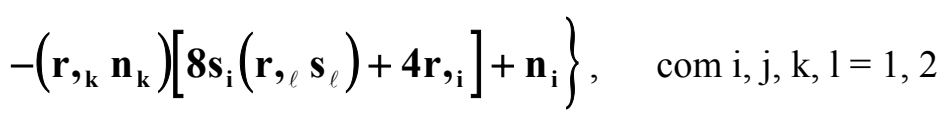

\subsection{Equação Integral para um Ponto do Contorno da Placa}

No caso do ponto pertencer ao contorno, o mesmo será denotado por Q. Assim, a fim de escrever-se a equação (3.19) para o ponto $\mathbf{Q}$, torna-se o mesmo interior ao domínio pelo acréscimo de um contorno circular $\Gamma_{\xi}$, centrado em $\mathbf{Q}$, com raio $\xi$, e pela retirada da parcela $\bar{\Gamma}$ do contorno, como é indicado na figura (3.4). O novo contorno será dado por $\Gamma-\bar{\Gamma}+\Gamma_{\xi}$ e o ponto $\mathbf{Q}$ será do contorno quando o raio $\xi$ e o contorno $\bar{\Gamma}$ tenderem à zero. Portanto, o deslocamento $\mathbf{w}(\mathbf{Q})$ do ponto $\mathbf{Q}$ será calculado a partir da equação (3.19), fazendo-se $\Gamma=\Gamma-\bar{\Gamma}+\Gamma_{\xi}$ e os limites de $\xi$ e $\bar{\Gamma}$ tenderem à zero, como é mostrado a seguir: 


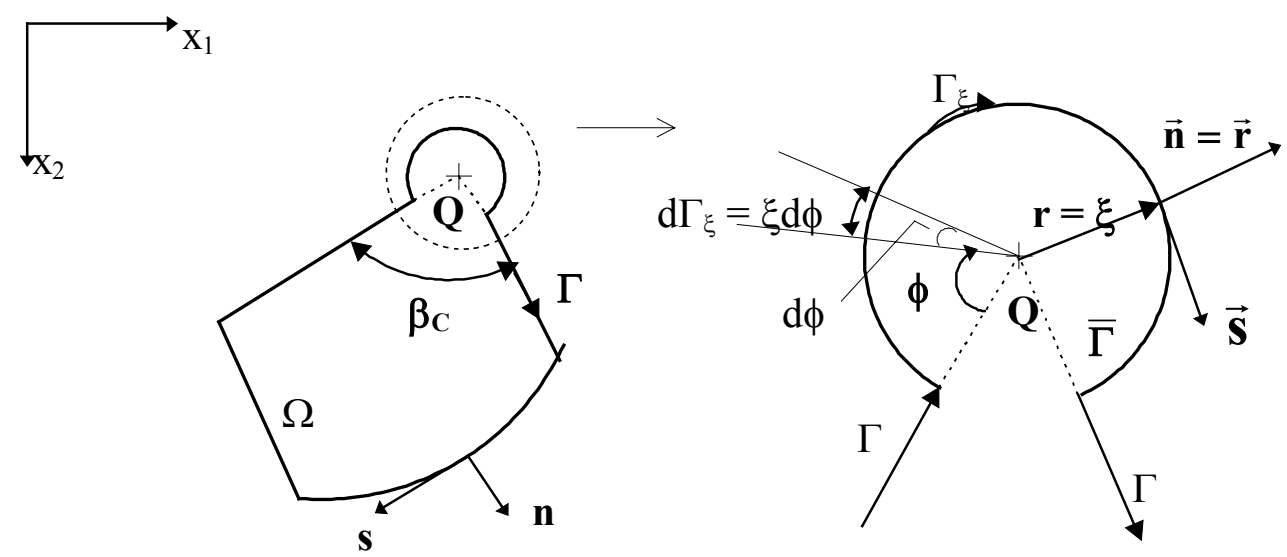

FIGURA 3.4 - Contorno Circular Acrescido a um ponto $\mathbf{Q}$ de um Canto da Placa

Da figura (3.4), pode-se tirar as seguintes relações:

$$
\begin{aligned}
& \mathbf{r}=\mathbf{R}=\xi, \\
& \mathbf{r}, \mathbf{n}_{\mathbf{i}}=\mathbf{1}, \\
& \mathbf{r}, \mathbf{s}_{\mathbf{i}}=\mathbf{0}, \\
& \mathbf{d} \Gamma_{\xi}(\mathbf{P})=\xi \mathbf{d} \phi .
\end{aligned}
$$

Desse modo, a equação integral do deslocamento do ponto $\mathbf{Q}$ resulta em:

$$
\begin{aligned}
& \mathbf{w}(\mathbf{Q})+\lim _{\Gamma \rightarrow 0} \int_{\Gamma-\bar{\Gamma}}\left(\mathbf{V}_{\mathbf{n}}^{*}(\mathbf{Q}, \mathbf{P}) \mathbf{w}(\mathbf{P})-\mathbf{M}_{\mathrm{nn}}^{*}(\mathbf{Q}, \mathbf{P}) \frac{\partial \mathbf{w}}{\partial \mathbf{n}}(\mathbf{P})\right) \mathbf{d} \Gamma(\mathbf{P})+ \\
&+\lim _{\xi \rightarrow 0} \int_{\Gamma_{\xi}}\left(\mathbf{V}_{\mathbf{n}}^{*}(\mathbf{Q}, \mathbf{P}) \mathbf{w}(\mathbf{P})-\mathbf{M}_{\mathrm{nn}}^{*}(\mathbf{Q}, \mathbf{P}) \frac{\partial \mathbf{w}}{\partial \mathbf{n}}(\mathbf{P})\right) \mathbf{d} \Gamma_{\xi}(\mathbf{P})+\sum_{\mathrm{i}=1}^{\mathbf{N}_{\mathrm{c}}-1} \mathbf{R}_{\mathrm{ci}}^{*}(\mathbf{Q}, \mathbf{P}) \mathbf{w}_{\mathrm{ci}}(\mathbf{P})+ \\
&+\lim _{\xi \rightarrow 0}\left[\mathbf{R}_{\mathrm{c} \lambda^{-}}^{*}(\mathbf{Q}, \mathbf{P}) \mathbf{w}_{\mathrm{c} \lambda^{-}}(\mathbf{P})\right]+\lim _{\xi \rightarrow 0}\left[\mathbf{R}_{\mathrm{c} \lambda^{+}}^{*}(\mathbf{Q}, \mathbf{P}) \mathbf{w}_{\mathrm{c} \lambda^{+}}(\mathbf{P})\right]= \\
&=\lim _{\bar{\Gamma} \rightarrow 0} \int_{\Gamma-\bar{\Gamma}}\left(\mathbf{V}_{\mathbf{n}}(\mathbf{P}) \mathbf{w}^{*}(\mathbf{Q}, \mathbf{P})-\mathbf{M}_{\mathrm{nn}}(\mathbf{P}) \frac{\partial \mathbf{w}^{*}}{\partial \mathbf{n}}(\mathbf{Q}, \mathbf{P})\right) \mathbf{d} \Gamma(\mathbf{P})+ \\
&+\lim _{\xi \rightarrow 0} \int_{\Gamma_{\xi}}\left(\mathbf{V}_{\mathbf{n}}(\mathbf{P}) \mathbf{w}^{*}(\mathbf{Q}, \mathbf{P})-\mathbf{M}_{\mathrm{nn}}(\mathbf{P}) \frac{\partial \mathbf{w}^{*}}{\partial \mathbf{n}}(\mathbf{Q}, \mathbf{P})\right) \mathbf{d} \Gamma_{\xi}(\mathbf{P})+ \\
&+\sum_{\mathrm{i}=1}^{\mathrm{N}_{\mathrm{c}}-1} \mathbf{R}_{\mathrm{ci}}(\mathbf{P}) \mathbf{w}_{\mathrm{ci}}^{*}(\mathbf{Q}, \mathbf{P})+\lim _{\xi \rightarrow 0}\left[\mathbf{R}_{\mathrm{c} \lambda^{-}}(\mathbf{Q}, \mathbf{P}) \mathbf{w}_{\mathrm{c} \lambda^{-}}^{*}(\mathbf{P})\right]+
\end{aligned}
$$




$$
+\lim _{\xi \rightarrow 0}\left[\mathbf{R}_{\mathrm{c} \lambda^{+}}(\mathbf{Q}, \mathbf{P}) \mathbf{w}_{\mathbf{c} \lambda^{+}}^{*}(\mathbf{P})\right]+\int_{\Omega_{\mathrm{g}}}\left(\mathbf{g}(\mathbf{p}) \mathbf{w}^{*}(\mathbf{Q}, \mathbf{p})\right) \mathbf{d} \Omega_{\mathrm{g}}(\mathbf{p})
$$

Os limites de $\bar{\Gamma} \rightarrow \mathbf{0}$ das integrais sobre o contorno $(\Gamma-\bar{\Gamma})$ na equação anterior são iguais ao valor principal das mesmas. Logo, pode-se escrever por definição que:

$$
\begin{aligned}
& \lim _{\bar{\Gamma} \rightarrow 0} \int_{\Gamma-\bar{\Gamma}}\left(V_{n}^{*}(\mathbf{Q}, \mathbf{P}) \mathbf{w}(\mathbf{P})-\mathbf{M}_{\mathrm{nn}}^{*}(\mathbf{Q}, \mathbf{P}) \frac{\partial \mathbf{w}}{\partial \mathbf{n}}(\mathbf{P})\right) \mathbf{d} \Gamma(\mathbf{P})= \\
& =\int_{\Gamma}\left(\mathbf{V}_{\mathbf{n}}^{*}(\mathbf{Q}, \mathbf{P}) \mathbf{w}(\mathbf{P})-\mathbf{M}_{\mathrm{nn}}^{*}(\mathbf{Q}, \mathbf{P}) \frac{\partial \mathbf{w}}{\partial \mathbf{n}}(\mathbf{P})\right) \mathbf{d} \Gamma(\mathbf{P}) \\
& \lim _{\bar{\Gamma} \rightarrow 0} \int_{\Gamma-\bar{\Gamma}}\left(\mathbf{V}_{\mathbf{n}}(\mathbf{P}) \mathbf{w}^{*}(\mathbf{Q}, \mathbf{P})-\mathbf{M}_{\mathrm{nn}}(\mathbf{P}) \frac{\partial \mathbf{w}^{*}}{\partial \mathbf{n}}(\mathbf{Q}, \mathbf{P})\right) \mathbf{d} \Gamma(\mathbf{P})= \\
& =\int_{\Gamma}\left(\mathbf{V}_{\mathbf{n}}(\mathbf{P}) \mathbf{w}^{*}(\mathbf{Q}, \mathbf{P})-\mathbf{M}_{\mathrm{nn}}(\mathbf{P}) \frac{\partial \mathbf{w}^{*}}{\partial \mathbf{n}}(\mathbf{Q}, \mathbf{P})\right) \mathbf{d} \Gamma(\mathbf{P})
\end{aligned}
$$

A parcela referente à integral sobre o trecho $\Gamma_{\xi}$, pode ser rescrita substituindo-se $\mathbf{w}(\mathbf{P})$ por $(\mathrm{w}(\mathrm{P})-\mathrm{w}(\mathrm{Q})+\mathrm{w}(\mathrm{Q}))$ e $\partial \mathrm{w}(\mathrm{P}) / \partial \mathrm{n}$ por $(\partial \mathrm{w}(\mathrm{P}) / \partial \mathrm{n}-\partial \mathrm{w}(\mathrm{Q}) / \partial \mathrm{n}+\partial \mathrm{w}(\mathrm{Q}) / \partial \mathrm{n})$. Considerando-se válida a continuidade, ou, utilizando-se a condição de Hölder (JASWON \& SYMM, 1977), que é dada por:

$$
|\mathbf{w}(\mathbf{P})-\mathbf{w}(\mathbf{Q})| \leq \mathbf{C}_{1} \mathbf{r}^{\alpha_{1}(\mathbf{P}, \mathbf{Q})}
$$

e

$$
\left|\frac{\partial \mathbf{w}}{\partial \mathbf{n}}(\mathbf{P})-\frac{\partial \mathbf{w}}{\partial \mathbf{n}}(\mathbf{Q})\right| \leq \mathbf{C}_{2} \mathbf{r}^{\alpha_{2}(\mathbf{P}, \mathbf{Q})}
$$

onde $\mathbf{C}_{1}$ e $\mathbf{C}_{2}$ são constantes e $\mathbf{0}<\alpha_{\mathbf{i}} \leq \mathbf{1}$, com $\mathrm{i}=1,2$, as parcelas (w(P) - w(Q)) e $(\partial \mathrm{w}(\mathrm{P}) / \partial \mathrm{n}-\partial \mathrm{w}(\mathrm{Q}) / \partial \mathrm{n})$ se anulam. Considerando-se ainda que os valores de $\mathbf{w}(\mathbf{Q})$ e $\partial \mathbf{w}(\mathbf{Q}) / \partial \mathbf{n}$ são valores do domínio e portanto, não variam ao longo do contorno $\Gamma_{\xi}$, tem-se que: 


$$
\begin{aligned}
& \lim _{\xi \rightarrow 0} \int_{\Gamma_{\xi}}\left(V_{n}^{*}(\mathbf{Q}, \mathbf{P}) \mathbf{w}(\mathbf{P})-\mathbf{M}_{\mathrm{nn}}^{*}(\mathbf{Q}, \mathbf{P}) \frac{\partial \mathbf{w}}{\partial \mathbf{n}}(\mathbf{P})\right) \mathbf{d} \Gamma_{\xi}(\mathbf{P})= \\
& =\mathbf{w}(\mathbf{Q}) \lim _{\xi \rightarrow 0} \int_{\Gamma_{\xi}} \mathbf{V}_{\mathbf{n}}^{*}(\mathbf{Q}, \mathbf{P}) \mathbf{d} \Gamma_{\xi}(\mathbf{P})-\frac{\partial \mathbf{w}}{\partial \mathbf{n}}(\mathbf{Q}) \lim _{\xi \rightarrow 0} \int_{\Gamma_{\xi}} \mathbf{M}_{\mathrm{nn}}^{*}(\mathbf{Q}, \mathbf{P}) \mathbf{d} \Gamma_{\xi}(\mathbf{P})
\end{aligned}
$$

Substituindo-se em (3.64) os valores de $\mathbf{V}_{\mathbf{n}}^{*}(\mathbf{Q}, \mathbf{P})$ e $\mathbf{M}_{n \mathbf{n}}^{*}(\mathbf{Q}, \mathbf{P})$, dados em (3.32) e (3.30), respectivamente, e considerando-se as equações (3.55), (3.56), (3.57) e (3.58), temse:

$$
\begin{aligned}
& \lim _{\xi \rightarrow 0} \int_{\Gamma_{\xi}}\left(V_{n}^{*}(\mathbf{Q}, \mathbf{P}) \mathbf{w}(\mathbf{P})-\mathbf{M}_{\mathrm{nn}}^{*}(\mathbf{Q}, \mathbf{P}) \frac{\partial \mathbf{w}}{\partial \mathbf{n}}(\mathbf{P})\right) \mathbf{d} \Gamma_{\xi}(\mathbf{P})= \\
& =\mathbf{w}(\mathbf{Q}) \lim _{\xi \rightarrow 0} \int_{0}^{2 \pi-\beta_{\mathrm{c}}}-\frac{1}{2 \pi \xi} \xi \mathbf{d} \phi+\frac{\partial \mathbf{w}(\mathbf{q})}{\partial \mathbf{n}} \lim _{\xi \rightarrow 0} \int_{0}^{2 \pi-\beta_{\mathrm{c}}} \frac{1}{4 \pi}[(1+v) \ln \xi+1] \xi \mathbf{d} \phi= \\
& =-\frac{2 \pi-\beta_{\mathbf{c}}}{2 \pi} \mathbf{w}(\mathbf{Q})
\end{aligned}
$$

onde $\beta_{\mathrm{c}}$ é o ângulo interno do canto da placa, indicado na figura (3.4).

Analogamente, as demais integrais sobre o contorno $\Gamma_{\xi}$ da equação (3.59) conduzem a valores nulos, assim como ocorre com os limites das parcelas que envolvem as reações de canto $\mathbf{R}_{\mathbf{c}}$, isto é:

$$
\begin{gathered}
\sum_{\mathrm{i}=1}^{\mathrm{N}_{\mathrm{c}}-1} \mathbf{R}_{\mathrm{ci}}^{*}(\mathbf{Q}, \mathbf{P}) \mathbf{w}_{\mathrm{ci}}(\mathbf{P})+\lim _{\xi \rightarrow 0}\left[\mathbf{R}_{\mathrm{c} \lambda^{-}}^{*}(\mathbf{Q}, \mathbf{P}) \mathbf{w}_{\mathrm{c} \lambda^{-}}(\mathbf{P})\right]+\lim _{\xi \rightarrow 0}\left[\mathbf{R}_{\mathrm{c} \lambda^{+}}^{*}(\mathbf{Q}, \mathbf{P}) \mathbf{w}_{\mathrm{c} \lambda^{+}}(\mathbf{P})\right]= \\
=\sum_{\mathrm{i}=1}^{\mathrm{N}_{\mathrm{c}}} \mathbf{R}_{\mathrm{ci}}^{*}(\mathbf{Q}, \mathbf{P}) \mathbf{w}_{\mathrm{ci}}(\mathbf{P})
\end{gathered}
$$

Portanto, a equação (3.59), que representa a equação integral de um ponto do contorno, pode ser escrita da seguinte forma: 


$$
\begin{aligned}
& \mathbf{K}(\mathbf{Q}) \mathbf{w}(\mathbf{Q})+\int_{\Gamma}\left(\mathbf{V}_{\mathrm{n}}^{*}(\mathbf{Q}, \mathbf{P}) \mathbf{w}(\mathbf{P})-\mathbf{M}_{\mathrm{nn}}^{*}(\mathbf{Q}, \mathbf{P}) \frac{\partial \mathbf{w}}{\partial \mathbf{n}}(\mathbf{P})\right) \mathbf{d} \Gamma(\mathbf{P})+ \\
& +\sum_{\mathrm{i}=1}^{\mathbf{N}_{\mathrm{c}}} \mathbf{R}_{\mathrm{ci}}^{*}(\mathbf{Q}, \mathbf{P}) \mathbf{w}_{\mathrm{ci}}(\mathbf{P})=\int_{\Gamma}\left(\mathbf{V}_{\mathrm{n}}(\mathbf{P}) \mathbf{w}^{*}(\mathbf{Q}, \mathbf{P})-\mathbf{M}_{\mathrm{nn}}(\mathbf{P}) \frac{\partial \mathbf{w}^{*}}{\partial \mathrm{n}}(\mathbf{Q}, \mathbf{P})\right) \mathbf{d} \Gamma(\mathbf{P})+ \\
& +\sum_{\mathrm{i}=1}^{\mathbf{N}_{\mathrm{c}}} \mathbf{R}_{\mathrm{ci}}(\mathbf{P}) \mathbf{w}_{\mathrm{ci}}^{*}(\mathbf{Q}, \mathbf{P})+\int_{\Omega_{\mathrm{g}}}\left(\mathbf{g}(\mathbf{P}) \mathbf{w}^{*}(\mathbf{Q}, \mathbf{p})\right) \mathbf{d} \Omega_{\mathrm{g}}(\mathbf{p})
\end{aligned}
$$

onde: $\quad \mathbf{K}(\mathbf{Q})=\frac{\boldsymbol{\beta}_{\mathbf{c}}}{2 \pi} \quad$ se o ponto $\mathbf{Q}$ coincide com um canto,

$\mathbf{K}(\mathbf{Q})=\frac{\pi}{2 \pi}=\frac{1}{\mathbf{2}} \quad$ se o ponto $\mathbf{Q}$ não coincide com um canto.

Derivando-se (3.67) em relação a uma coordenada genérica $\mathbf{m}$, pode-se obter a equação integral da derivada do deslocamento $\partial \mathbf{w}(\mathbf{Q}) / \partial \mathbf{m}$, para um ponto do contorno, como é mostrado no trabalho de PAIVA (1987).

\subsection{Transformação das Integrais de Domínio do Carregamento em Integrais de Contorno}

Para a aplicação do Método dos Elementos de Contorno é conveniente transformar as integrais de domínio que aparecem nas equações (3.19) e (3.67), correspondentes às influências do carregamento distribuído na área $\Omega_{\mathrm{g}}$, em integrais sobre o contorno $\Gamma_{\mathrm{g}}$, onde a mesma está distribuída. Para isto, considere-se a figura (3.5), na qual estão representados a região $\Omega_{\mathrm{g}}$, seu contorno $\Gamma_{\mathrm{g}}$ e o ponto de carregamento $\mathbf{q}$ e a partir da qual, obtêm-se as seguintes relações:

$$
\begin{aligned}
& \mathrm{d} \theta=\frac{r_{,_{i}} n_{i}}{R} d \Gamma_{g} \\
& d \Omega_{g}=\operatorname{rdrd} \theta=\operatorname{rdr} \frac{r,_{i} n_{i}}{R} d \Gamma_{g}
\end{aligned}
$$

onde $\mathbf{R}$ é o valor de $\mathbf{r}$ para um ponto qualquer do contorno $\Gamma_{\mathbf{g}}$. 


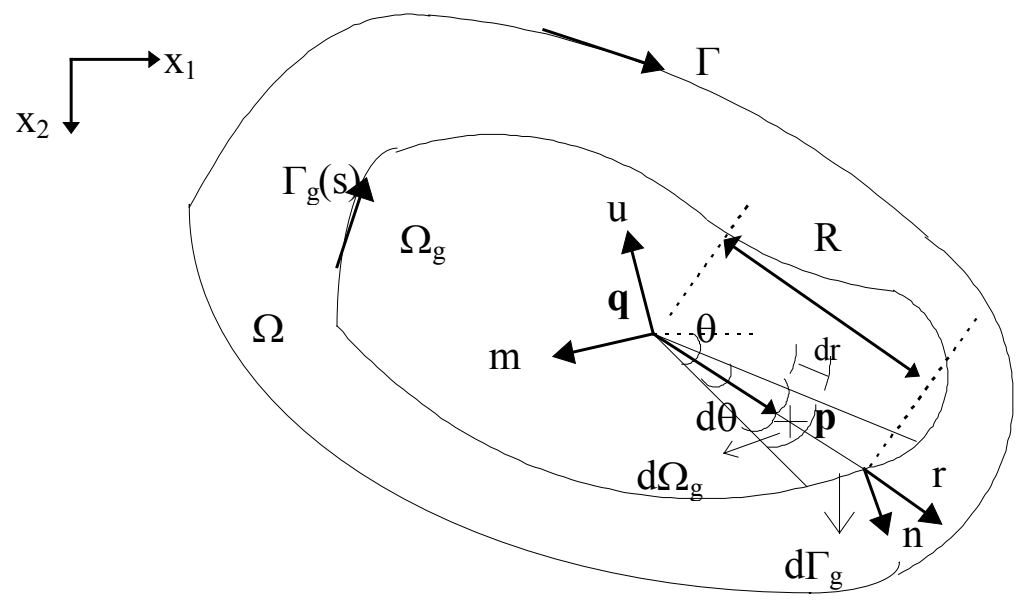

FIGURA 3.5 - Região Carregada $\Omega_{\mathrm{g}}$ de uma Placa de Domínio $\Omega$

Fazendo-se a mudança de coordenadas dada por (3.69), tem-se que:

$$
\int_{\Omega_{\mathrm{g}}} \mathbf{g}(\mathbf{p}) \mathbf{w}^{*}(\mathbf{Q}, \mathbf{p}) \mathrm{d} \Omega_{\mathrm{g}}(\mathbf{p})=\int_{\Gamma_{\mathrm{g}}}\left(\int_{0}^{\mathrm{R}} \mathbf{g}(\mathbf{p}) \mathbf{w}^{*}(\mathbf{q}, \mathbf{p}) \mathbf{r d r}\right) \frac{\mathbf{r},,_{\mathrm{i}} \mathbf{n}_{\mathrm{i}}}{\mathbf{R}} \mathrm{d} \Gamma_{\mathrm{g}}
$$

Admitindo-se que a carga $\mathbf{g}(\mathbf{p})$ varie linearmente na região $\Omega_{\mathrm{g}}$ e considerando-se as relações entre os sistemas de coordenadas cartesianas e polares, com origem em q ( ver figura 3.2), dadas por:

$$
\begin{aligned}
& x_{1}(p)=x_{1}(q)+r \cos \theta \\
& x_{2}(p)=x_{2}(q)+r \operatorname{sen} \theta
\end{aligned}
$$

pode-se escrever $\mathbf{g}(\mathbf{p})$ da seguinte maneira:

$$
\mathbf{g}(\mathbf{p})=\operatorname{Ar} \cos \theta+\operatorname{Br} \operatorname{sen} \theta+\mathbf{g}(\mathbf{q})
$$

onde $\mathbf{g}(\mathbf{q})=\mathbf{A x _ { 1 }}(\mathbf{q})+\mathbf{B} \mathbf{x}_{2}(\mathbf{q})+\mathbf{C}$, é uma constante, correspondente ao valor de $\mathbf{g}$ no ponto q.

Substituindo-se (3.71) e $\mathbf{w}^{*}(\mathbf{q}, \mathbf{p})$, dado por (3.25), em (3.70) e fazendo-se a integração em relação a $\mathbf{r}$, obtém-se: 


$$
\begin{aligned}
& \int_{\Omega_{g}} g(p) w^{*}(Q, p) d \Omega_{g}(p)=\frac{g(q)}{32 \pi D} \int_{\Gamma_{g}} R^{3}\left(\ln R-\frac{3}{4}\right) r,,_{i} n_{i} d \Gamma_{g}+ \\
& +\frac{1}{40 \pi D} \int_{\Gamma_{g}} R^{4}\left(\ln R-\frac{7}{10}\right)(A \cos \theta+B \operatorname{sen} \theta) r,_{i} n_{i} d \Gamma_{g}
\end{aligned}
$$

A integral de domínio sobre a região carregada $\Omega_{\mathrm{g}}$, pode ainda ser calculada,

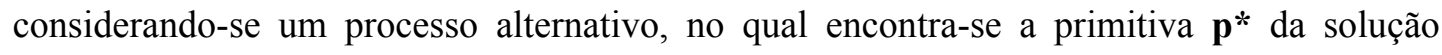
fundamental $\mathbf{w}^{*}$, tal que:

$$
\nabla^{2} \mathbf{p}^{*}=\mathbf{w} *
$$

Integrando-se por partes duas vezes, pode-se escrever:

$$
\begin{aligned}
\int_{\Omega_{\mathrm{g}}} \mathbf{g}(\mathbf{p}) \mathbf{w} * d \Omega= & \int_{\Omega_{\mathrm{g}}} \mathbf{g}(\mathbf{p}) \nabla^{2} \mathbf{p} * d \Omega=\int_{\Gamma_{\mathrm{g}}} \mathrm{g}(\mathbf{p}) \frac{\partial \mathbf{p} *}{\partial \mathbf{n}} \mathrm{d} \Gamma+ \\
& -\int_{\Gamma_{\mathrm{g}}} \mathbf{p} * \frac{\partial \mathrm{g}(\mathbf{p})}{\partial \mathbf{n}} \mathrm{d} \Gamma+\int_{\Omega_{\mathrm{g}}} \mathrm{p}^{*} \nabla^{2} \mathbf{g}(\mathbf{p}) \mathrm{d} \Omega
\end{aligned}
$$

O termo de domínio torna-se nulo para $\mathbf{g}$ linear. No caso de $\mathbf{g}$ ser de grau maior, esse termo será eliminado repetindo-se o processo com novas primitivas. 


\section{MÉtodo DOS ELEMENTOS DE CONTORNO APLICADO A PROBLEMAS DE PLACAS SUJEITAS A CARGAS TRANSVERSAIS}

\subsection{Introdução}

A solução analítica das integrais, que fornece os resultados exatos, está restrita a poucos problemas, onde alguma simplificação é possível para se ter a redução do número de graus de liberdade a um número finito. Assim é necessário, a utilização de métodos numéricos para a obtenção de soluções aproximadas.

Neste trabalho, será usado o Método dos Elementos de Contorno, que consiste na divisão do contorno da placa em segmentos, denominados elementos de contorno, sobre os quais as variáveis $\mathbf{w}, \partial \mathbf{w} / \partial \mathbf{n}, \mathbf{V}_{\mathbf{n}}$ e $\mathbf{M}_{\mathbf{n}}$ são aproximadas por funções interpoladoras, definidas em função de pontos previamente escolhidos em cada elemento, ditos nós ou pontos nodais. Assim, as equações integrais transformam-se em equações algébricas, que são escritas em função dos valores das variáveis nos nós do contorno, e portanto, são denominados de valores nodais.

Tem-se duas incógnitas por nó, pois duas das quatro variáveis nodais são dadas como condição de contorno. Logo, escrevendo-se duas equações para cada nó do contorno, obtémse um sistema de equações lineares, onde as incógnitas são os deslocamentos e esforços dos nós do contorno. Impondo-se as condições de contorno ao sistema, obtém-se as incógnitas do contorno e então, com os valores das mesmas escrevem-se as equações em cada ponto interno, obtendo-se as incógnitas dos pontos do domínio. 


\section{2 - Discretização das Equações Integrais}

A discretização das equações integrais é obtida através da divisão do contorno em segmentos, conforme indicado na figura (4.1). O número e a forma dos elementos devem ser escolhidos de tal forma que representem adequadamente o contorno real da placa, de maneira exata ou aproximada.
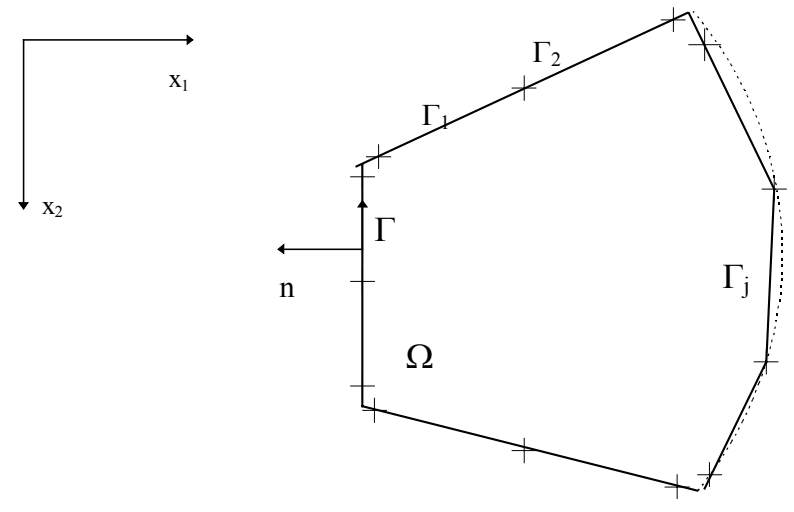

FIGURA 4.1 - Discretização do Contorno da Placa

\subsubsection{Aproximação da Geometria do Elemento}

Nesse trabalho, a geometria dos elementos será representada por uma função linear, isto é os elementos serão retos. Como as integrais são resolvidas numericamente, é conveniente expressar as coordenadas de cada ponto $\mathbf{P}$ em função de coordenadas locais homogêneas $\xi$ como é mostrado na figura (4.2).

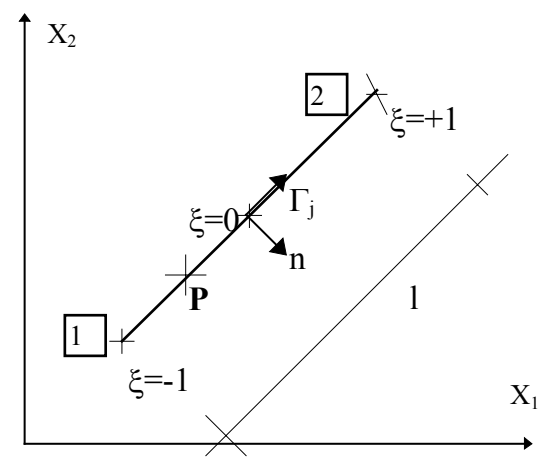

FIGURA 4.2 - Geometria do Elemento

onde: $\mathbf{1}$ é o nó inicial do elemento ao qual $\mathbf{P}$ pertence, 
2 é o nó final do elemento,

l é o comprimento do elemento,

$$
\begin{array}{rlr}
-1 & \leq \xi & \leq 1 \\
\Gamma & =\xi \frac{1}{2}, \\
\mathrm{~d} \Gamma & =\frac{1}{2} \mathrm{~d} \xi .
\end{array}
$$

Da figura (4.2), têm-se que as coordenadas do ponto $\mathbf{P}$ são dadas por:

$$
\begin{aligned}
& X_{1}(P)=\frac{X_{1}^{1}+X_{1}^{2}}{2}+\xi\left(\frac{X_{1}^{2}-X_{1}^{1}}{2}\right) \\
& X_{2}(P)=\frac{X_{2}^{1}+X_{2}^{2}}{2}+\xi\left(\frac{X_{2}^{2}-X_{2}^{1}}{2}\right)
\end{aligned}
$$

onde: $\mathbf{X}_{\mathbf{i}}^{\mathbf{N}}$ é a coordenada na direção $\mathbf{i}$ do nó $\mathbf{N}$.

As equações (4.2) podem ser escritas numa só equação matricial da seguinte forma:

$$
\left\{\begin{array}{l}
X_{1}(P) \\
X_{2}(P)
\end{array}\right\}=\left[\begin{array}{cccc}
\phi_{g 1}(P) & \phi_{g 2}(P) & 0 & 0 \\
0 & 0 & \phi_{g 1}(P) & \phi_{g 2}(P)
\end{array}\right]\left\{\begin{array}{l}
X_{1}^{1} \\
X_{1}^{2} \\
X_{2}^{1} \\
X_{2}^{2}
\end{array}\right\}
$$

onde: $\quad \phi_{\mathrm{gi}}$ são as funções interpoladoras que são dadas por:

$$
\begin{gathered}
\phi_{\mathrm{g} 1}(\mathrm{P})=\frac{1}{2}(1-\xi), \\
\phi_{\mathrm{g} 2}(\mathrm{p})=\frac{1}{2}(1+\xi),
\end{gathered}
$$




$$
\underset{\sim}{X^{N}}=\left\{\begin{array}{l}
X_{1}^{1} \\
X_{1}^{2} \\
X_{2}^{1} \\
X_{2}^{2}
\end{array}\right\} \text { é o vetor dos valores nodais das coordenadas. }
$$

Assim, fazendo-se a discretização do contorno em $\mathbf{N}_{\mathbf{e}}$ elementos e a mudança de coordenadas cartesianas para coordenadas homogêneas, dada em (4.1.b), tem-se:

$$
\int_{\Gamma} \mathbf{F}(\Gamma) \mathrm{d} \Gamma=\sum_{\mathrm{j}=1}^{\mathrm{N}_{\mathrm{e}}} \int_{-1 / 2}^{1 / 2} \mathbf{F}\left(\Gamma_{\mathrm{j}}\right) \mathrm{d} \Gamma_{\mathrm{j}}=\frac{1}{2} \sum_{j=1}^{\mathrm{N}_{\mathrm{e}}} \int_{-1}^{1} \mathbf{F}(\xi) \mathrm{d} \xi
$$

\subsubsection{Aproximação das Variáveis do problema}

As variáveis são aproximadas por funções polinomiais quadráticas, portanto são necessário três pontos nodais em cada elemento (ver figura 4.3). As funções interpoladoras são definidas para os nós das extremidades do elemento e para o nó central do mesmo. Assim, em coordenadas homogêneas, o nó $\mathbf{1}$ tem a coordenada $\xi=\xi_{1}=-\mathbf{1}$, o nó $\mathbf{2}$ $\xi=\xi_{2}=\mathbf{0}$ e o nó $3 \xi=\xi_{3}=1$ (Figura 4.3). As funções de forma $\phi_{\mathrm{i}}$, que são as funções de interpolação, são definidas de tal forma que sejam iguais a um $\left(\phi_{\mathbf{i}}=\mathbf{1}\right)$ para o nó i e nulas para os demais nós, como é mostrado na figura (4.3).
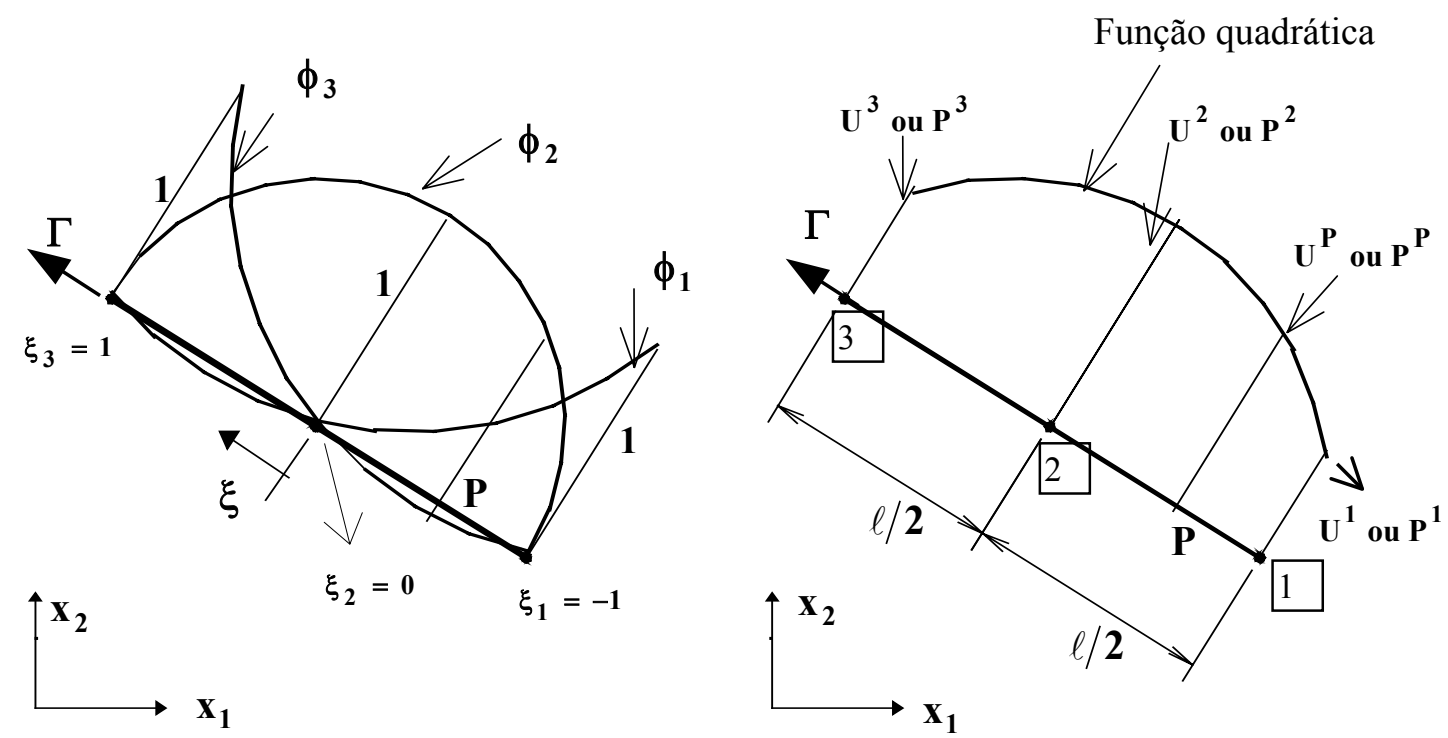

FIGURA 4.3 - Funções de Forma em Aproximação Quadrática das Variáveis 
Desse modo, pode-se expressar os vetores de deslocamentos $\mathbf{u}$ e de esforços $\mathbf{p}$ de um ponto $\mathbf{P}$ qualquer do elemento, da seguinte forma:

$$
\begin{aligned}
& \underset{\sim}{\mathbf{u}(P)}={\underset{\sim}{\phi}}^{\mathrm{T}}(\mathbf{P}) \underset{\sim}{\mathbf{\sim}^{\mathrm{N}}} \\
& \underset{\sim}{\mathbf{p}(\mathbf{P})}={\underset{\sim}{\phi}}^{\mathrm{T}}(\mathbf{P}) \underset{\sim}{\mathbf{P}^{\mathrm{N}}}
\end{aligned}
$$

ou, explicitamente:

$$
\begin{aligned}
& \underset{\sim}{\mathbf{u}}(\mathbf{P})=\left\{\begin{array}{l}
\mathbf{u}_{1}(\mathbf{P}) \\
\mathbf{u}_{2}(\mathbf{P})
\end{array}\right\}=\left[\begin{array}{cccccc}
\phi_{1}(\mathbf{P}) & \mathbf{0} & \phi_{2}(\mathbf{P}) & \mathbf{0} & \phi_{3}(\mathbf{P}) & \mathbf{0} \\
\mathbf{0} & \phi_{1}(\mathbf{P}) & \mathbf{0} & \phi_{2}(\mathbf{P}) & \mathbf{0} & \phi_{3}(\mathbf{P})
\end{array}\right]\left\{\begin{array}{l}
\mathbf{U}_{1}^{1} \\
\mathbf{U}_{2}^{1} \\
\mathbf{U}_{1}^{2} \\
\mathbf{U}_{2}^{2} \\
\mathbf{U}_{1}^{3} \\
\mathbf{U}_{2}^{3}
\end{array}\right\} \\
& \underset{\sim}{\mathbf{p}(\mathbf{P})}=\left\{\begin{array}{l}
\mathbf{p}_{1}(\mathbf{P}) \\
\mathbf{p}_{2}(\mathbf{P})
\end{array}\right\}=\left[\begin{array}{cccccc}
\phi_{1}(\mathbf{P}) & \mathbf{0} & \phi_{2}(\mathbf{P}) & \mathbf{0} & \phi_{3}(\mathbf{P}) & \mathbf{0} \\
\mathbf{0} & \phi_{1}(\mathbf{P}) & \mathbf{0} & \phi_{2}(\mathbf{P}) & \mathbf{0} & \phi_{3}(\mathbf{P})
\end{array}\right]\left\{\begin{array}{l}
\mathbf{P}_{1}^{1} \\
\mathbf{P}_{2}^{1} \\
\mathbf{P}_{1}^{2} \\
\mathbf{P}_{2}^{2} \\
\mathbf{P}_{1}^{3} \\
\mathbf{P}_{2}^{3}
\end{array}\right\}
\end{aligned}
$$

onde: $\quad \mathbf{U}_{\mathbf{i}}^{\mathbf{N}}$ e $\mathbf{P}_{\mathbf{i}}^{\mathbf{N}}$ são os deslocamentos e esforços na direção i do nó $\mathbf{N}$,

$\mathbf{u}_{1}=\mathbf{w}=$ flecha,

$\mathbf{u}_{2}=\frac{\partial \mathbf{w}}{\partial \mathbf{n}}=$ rotação,

$\mathbf{p}_{1}=\mathbf{V}_{\mathbf{n}}=$ cortante equivalente,

$\mathbf{p}_{2}=\mathbf{M}_{\mathbf{n}}=$ momento na direção normal ao contorno,

$\phi_{\mathbf{i}}$ são as funções interpoladoras quadráticas, dadas por:

$$
\begin{aligned}
& \phi_{1}(P)=-\frac{1}{2} \xi(1-\xi), \\
& \phi_{2}(P)=1-\xi^{2}, \\
& \phi_{3}(P)=\frac{1}{2} \xi(1+\xi),
\end{aligned}
$$


As equações (4.10), são obtidas das funções de forma escritas para um caso geral, onde adota-se $\xi_{1}=-\mathbf{1}$ e $\xi_{3}=1$. Assim, para um caso geral as funções são dadas por:

$$
\begin{aligned}
\phi_{1}(\mathbf{P}) & =\frac{\xi\left(\xi_{3}-\xi\right)}{\xi_{1}\left(\xi_{3}-\xi_{1}\right)} \\
\phi_{2}(\mathbf{P}) & =1-\frac{\xi_{3}+\xi_{1}}{\xi_{1} \xi_{3}} \xi+\frac{1}{\xi_{1} \xi_{3}} \xi^{2} \\
\phi_{3}(\mathbf{P}) & =\frac{\xi_{\left(\xi_{1}-\xi\right)}}{\xi_{3}\left(\xi_{1}-\xi_{3}\right)}
\end{aligned}
$$

No caso em que não haja descontinuidade nos valores das variáveis entre dois elementos adjacentes, os elementos são ditos contínuos, como é mostrado na figura (4.4).

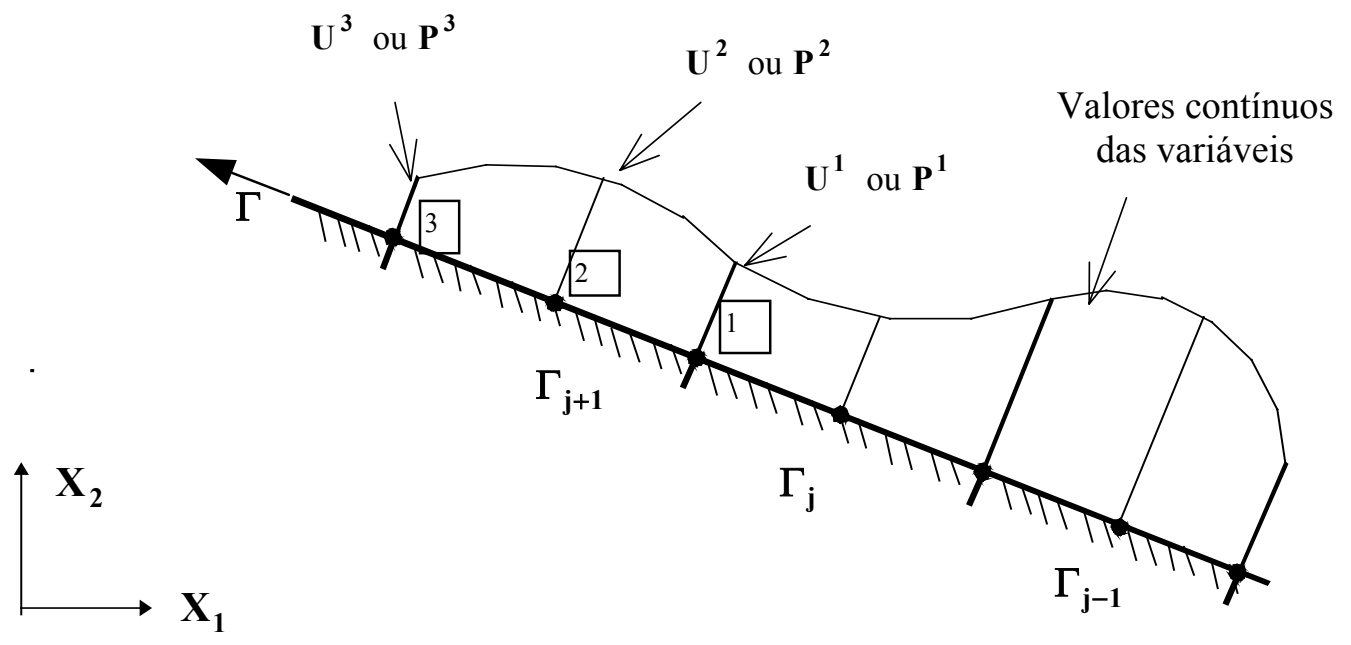

FIGURA 4.4 - Elementos Contínuos

Para representar a descontinuidade das variáveis, que ocorre em situações onde há variação repentina das condições de contorno entre dois elementos consecutivos, como ocorre nos cantos, definem-se nós duplos (figura 4.5), que são dois nós definidos com as mesmas coordenadas. Contudo, para ser possível representar a descontinuidade, deve-se escrever duas equações independentes para o ponto onde há descontinuidade. Uma das maneiras para se obter isso é mudando o ponto de colocação de lugar, isto é, as coordenadas do nó duplo são recalculadas, de tal forma que ele se torne interno ao elemento e não mais coincidente com sua extremidade (ver figura 4.5) e, então, escreve-se a equação para esse 
novo ponto. Isso pode se feito de duas maneiras: num primeiro caso, mantêm-se os nós extremos coincidentes com as extremidades do elemento (figura 4.5) e num segundo caso, o nó duplo deslocado coincide com um nó do elemento (figura 4.6). A integração numérica, no primeiro caso, é feita utilizando-se as funções interpoladoras indicadas em (4.10), isto é, considerando-se $\xi_{1}=-1$ e $\xi_{3}=1$ e, no segundo caso, têm-se que $\xi_{1} \neq-\mathbf{1}$ e/ou $\xi_{3} \neq \mathbf{1}$, e, portanto, utiliza-se as funções interpoladoras indicadas em (4.11). Entretanto, essa segunda alternativa não foi considerada nesse trabalho. Desse modo, o elemento que possuir um nó duplo será um elemento descontínuo.

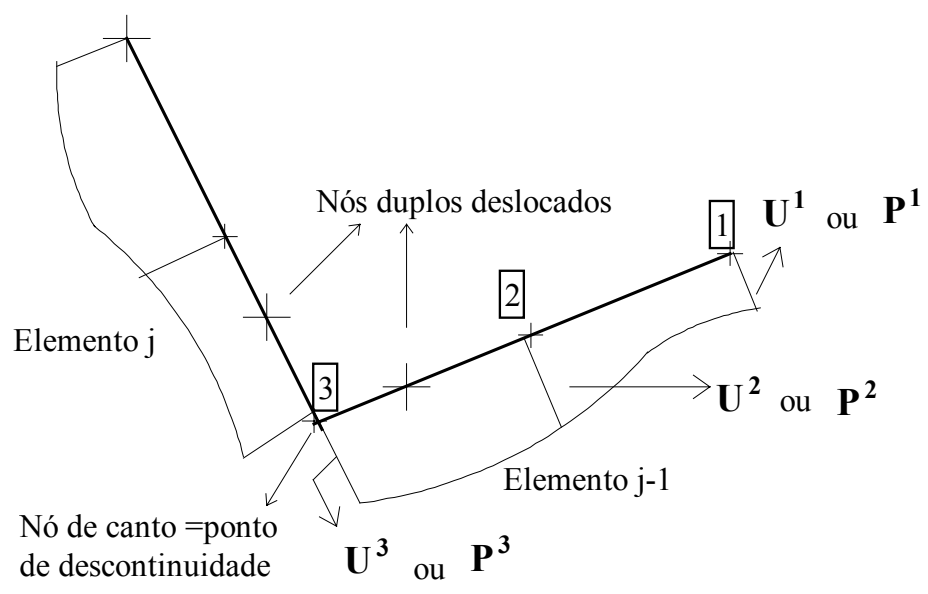

FIGURA 4.5 - Elementos Descontínuos
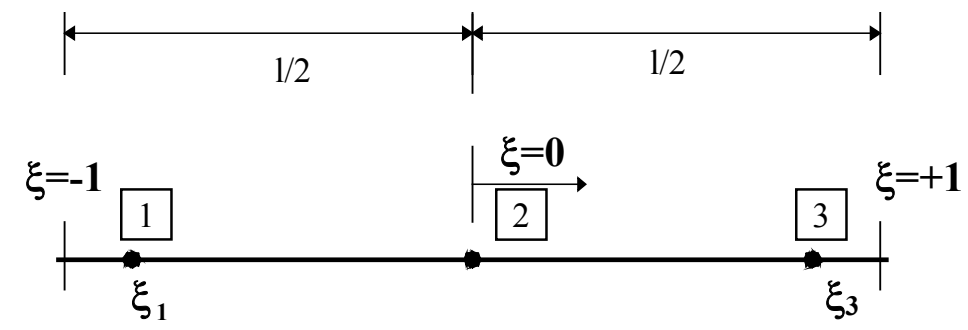

FIGURA 4.6 - Elemento de Contorno Descontínuo

O cálculo das coordenadas do nó duplo será feito a partir da equação (4.3), considerando-se $\mathbf{0 , 4} \leq|\xi| \leq \mathbf{0 , 6}$. O valor de $\xi$ será negativo se o ponto de colocação em consideração for o nó inicial do elemento e será positivo se o mesmo for o nó final. Adotouse esses limites para o valor de $\xi$, para que se tenha um afastamento conveniente entre os nós locais do elemento, a fim de evitar problemas de singularidades no sistema de equações, que 
ocorre quando se tem duas equações linearmente dependentes. Como as incógnitas estão expressas nas extremidades e no meio do elemento, tem-se a necessidade de se representar a flecha $\mathbf{w}$ do nó duplo deslocado $\mathbf{Q}$ em função dos valores nodais da flecha do elemento, ou seja:

$$
\mathbf{w}(\mathbf{Q})=\phi_{1} \mathbf{w}^{1}+\phi_{2} \mathbf{w}^{2}+\phi_{3} \mathbf{w}^{3}
$$

onde: $\mathbf{w}^{\mathbf{i}}$ é o deslocamento $\mathbf{w}$ do nó $\mathbf{i}$ do elemento,

$\phi_{\mathbf{i}}$ são as funções aproximadoras, dadas pelas equações (4.10).

\subsubsection{Transformações das Equações Integrais em Equações Algébricas}

A equação integral do deslocamento de um ponto $\mathbf{Q}$ dada por (3.67) pode ser escrita da seguinte maneira:

$$
\begin{gathered}
\mathbf{K}(\mathbf{Q}) \mathbf{w}(\mathbf{Q})+\int_{\Gamma} \underset{\sim}{\mathbf{p}^{*}}(\mathbf{Q}, \mathbf{P}) \underset{\sim}{\mathbf{u}}(\mathbf{P}) \mathrm{d} \Gamma(\mathbf{P})+\sum_{\mathrm{i}=1}^{\mathbf{N}_{\mathrm{c}}} \mathbf{R}_{\mathrm{ci}}^{*}(\mathbf{Q}, \mathbf{P}) \mathbf{w}_{\mathrm{ci}}(\mathbf{P})= \\
=\int_{\Gamma} \underset{\sim}{\mathbf{u}^{*}}\left(\mathbf{Q}, \underset{\sim}{\mathbf{P}} \underset{\sim}{\mathbf{p}}(\mathbf{P}) \mathrm{d} \Gamma(\mathbf{P})+\sum_{\mathrm{i}=1}^{\mathbf{N}_{\mathrm{c}}} \mathbf{R}_{\mathrm{ci}}(\mathbf{P}) \mathbf{w}_{\mathrm{ci}}^{*}(\mathbf{Q}, \mathbf{P})+\right. \\
\quad+\int_{\Omega_{\mathrm{g}}} \mathbf{g}(\mathbf{p}) \mathbf{w}^{*}(\mathbf{Q}, \mathbf{p}) \mathrm{d} \Omega_{\mathrm{g}}(\mathbf{p})
\end{gathered}
$$

onde: $\quad \mathbf{p}^{*}(\mathbf{Q}, \mathbf{P})=\left\{\mathbf{V}_{\mathbf{n}}^{*}(\mathbf{Q}, \mathbf{P})-\mathbf{M}_{\mathbf{n n}}^{*}(\mathbf{Q}, \mathbf{P})\right\}$

$$
\begin{aligned}
& \underset{\sim}{\mathbf{u}^{*}}(\mathbf{Q}, \mathbf{P})=\left\{\mathbf{w}^{*}(\mathbf{Q}, \mathbf{P})-\frac{\partial \mathbf{w}^{*}}{\partial \mathbf{n}}(\mathbf{Q}, \mathbf{P})\right\} \\
& \underset{\sim}{\mathbf{u}(\mathbf{P})}=\left\{\begin{array}{l}
\mathbf{u}_{1}(\mathbf{P}) \\
\mathbf{u}_{2}(\mathbf{P})
\end{array}\right\}=\left\{\begin{array}{c}
\mathbf{w}(\mathbf{P}) \\
\frac{\partial \mathbf{w}}{\partial \mathbf{n}}(\mathbf{P})
\end{array}\right\} \\
& \underset{\sim}{\mathbf{p}(\mathbf{P})}=\left\{\begin{array}{l}
\mathbf{p}_{1}(\mathbf{P}) \\
\mathbf{p}_{2}(\mathbf{P})
\end{array}\right\}=\left\{\begin{array}{c}
\mathbf{V}_{\mathbf{n}}(\mathbf{P}) \\
\mathbf{M}_{\mathbf{n}}(\mathbf{P})
\end{array}\right\}
\end{aligned}
$$

$\mathbf{V}_{\mathbf{n}}^{*}, \mathbf{M}_{\mathbf{n}}^{*}, \mathbf{w}^{*}$ e $\partial \mathbf{w}^{*} / \partial \mathbf{n}$ são dados, respectivamente, por (3.33), (3.30), (3.25) e (3.27). 
Fazendo-se a discretização do contorno em $\mathbf{N}_{\mathrm{e}}$ elementos, e substituindo-se as variáveis por suas aproximações em cada elemento dadas por (4.6) e (4.7), a equação (4.13) fica:

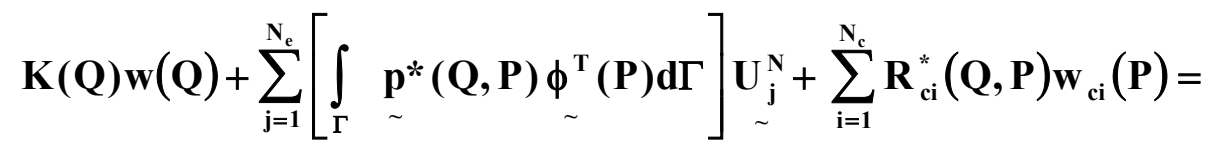

$$
\begin{aligned}
& =\sum_{\mathbf{j}=1}^{\mathrm{N}_{\mathrm{e}}}\left[\int_{\Gamma} \underset{\sim}{\mathbf{u}^{*}}(\mathbf{Q}, \mathbf{P}) \underset{\sim}{\phi^{\mathrm{T}}}(\mathbf{P}) \mathbf{d} \Gamma\right] \underset{\sim}{\mathbf{P}_{j}^{\mathrm{N}}}+\sum_{\mathrm{i}=1}^{\mathrm{N}_{\mathrm{c}}} \mathbf{R}_{\mathrm{ci}}(\mathbf{P}) \mathbf{w}_{\mathrm{ci}}^{*}(\mathbf{Q}, \mathbf{P})+ \\
& +\int_{\Omega_{\mathrm{g}}} \mathbf{g}(\mathbf{p}) \mathbf{w}^{*}(\mathbf{Q}, \mathbf{p}) \mathbf{d} \Omega_{\mathrm{g}}(\mathbf{p})
\end{aligned}
$$

Definindo:

$$
\begin{aligned}
& \underset{\sim}{\mathbf{h}^{\mathrm{j}}}(\mathbf{Q})=\int_{\Gamma} \underset{\sim}{\mathbf{p}^{*}}(\mathbf{Q}, \mathbf{P}) \phi_{\sim}^{\mathrm{T}}(\mathbf{P}) \mathrm{d} \Gamma, \\
& \underset{\sim}{\mathbf{g}^{\mathrm{j}}}(\mathbf{Q})=\int_{\Gamma} \underset{\sim}{\mathbf{u}^{*}}(\mathbf{Q}, \mathbf{P}) \underset{\sim}{\phi^{\mathrm{T}}}(\mathbf{P}) \mathrm{d} \Gamma \\
& \operatorname{e} \mathbf{t}(\mathbf{Q})=\int_{\Omega_{\mathrm{g}}} \mathbf{g}(\mathbf{p}) \mathbf{w}^{*}(\mathbf{Q}, \mathbf{p}) \mathbf{d} \Omega_{\mathrm{g}}(\mathbf{p})
\end{aligned}
$$

a equação (4.18) pode ser rescrita da seguinte forma:

$$
\begin{aligned}
& \mathbf{K}(\mathbf{Q}) \mathbf{w}(\mathbf{Q})+\sum_{j=1}^{N_{e}} \underset{\sim}{\mathbf{h}^{j}}(\mathbf{Q}) \mathbf{U}_{j}^{\mathbf{N}}+\sum_{i=1}^{N_{c}} \mathbf{R}_{\mathrm{ci}}^{*}(\mathbf{Q}, \mathbf{P}) \mathbf{w}_{\mathrm{ci}}(\mathbf{P})= \\
& =\sum_{\mathrm{j}=1}^{\mathrm{N}_{\mathrm{e}}} \underset{\sim}{\mathbf{g}^{\mathrm{j}}}(\mathbf{Q}) \underset{\sim}{\mathbf{P}_{\mathbf{j}}^{\mathrm{N}}}+\sum_{\mathrm{i}=1}^{\mathbf{N}_{\mathrm{c}}} \mathbf{R}_{\mathrm{ci}}(\mathbf{P}) \mathbf{w}_{\mathrm{ci}}^{*}(\mathbf{Q}, \mathbf{P})+\mathbf{t}(\mathbf{Q})
\end{aligned}
$$

Considerando-se o ponto de colocação em $\mathbf{Q}$, soma-se as influências ${\underset{\sim}{\mathbf{h}}}_{\mathbf{j}}(\mathbf{Q})$ e $\mathbf{g}^{\mathbf{j}}(\mathbf{Q})$ de todos os elementos, agrupando-se os coeficientes multiplicativos de um mesmo valor nodal, para todos os nós do contorno. Assim, pode-se escrever a equação (4.22) matricialmente: 


$$
\mathbf{K}(\mathbf{Q}) \mathbf{w}(\mathbf{Q})+\underset{\sim}{\hat{H}}(\mathbf{Q}) \underset{\sim}{\mathbf{U}}+\underset{\sim}{\mathbf{H}_{\mathbf{c}}}(\mathbf{Q}) \mathbf{w}_{\mathbf{c}}=\underset{\sim}{\mathbf{G}}(\mathbf{Q}) \underset{\sim}{\mathbf{P}}+\underset{\sim}{\mathbf{G}_{\mathbf{c}}}(\mathbf{Q}) \underset{\sim}{\mathbf{R}_{\mathbf{c}}}+\mathbf{T}(\mathbf{Q})
$$

onde:

- $\underset{\sim}{\mathbf{U}^{T}}=\left\{\begin{array}{llllllll}\mathbf{w}^{1} & \frac{\partial \mathbf{w}^{1}}{\partial \mathbf{n}} & \ldots & \mathbf{w}^{\mathrm{i}} & \frac{\partial \mathbf{w}^{\mathbf{i}}}{\partial \mathbf{n}} & \ldots & \mathbf{w}^{\mathbf{N}_{\mathbf{n}}} & \frac{\partial \mathbf{w}^{\mathbf{N}_{\mathbf{n}}}}{\partial \mathbf{n}}\end{array}\right\} \quad \begin{gathered}\text { é o vetor dos valores } \\ \text { on }\end{gathered}$ nodais dos deslocamentos do contorno da placa,

- $\underset{\sim}{\mathbf{P}^{\mathbf{T}}}=\left\{\begin{array}{llllllll}\mathbf{V}_{\mathbf{n}}^{1} & \mathbf{M}_{\mathbf{n}}^{1} & \ldots & \mathbf{V}_{\mathbf{n}}^{\mathrm{i}} & \mathbf{M}_{\mathbf{n}}^{\mathrm{i}} & \ldots & \mathbf{V}_{\mathbf{n}}^{\mathbf{N}_{\mathbf{n}}} & \mathbf{M}_{\mathbf{n}}^{\mathbf{N}_{\mathbf{n}}}\end{array}\right\}$ é o vetor dos valores nodais dos esforços do contorno da placa,

- $\quad \mathbf{w}_{\mathbf{c}}^{\mathbf{T}}=\left\{\begin{array}{lllll}\mathbf{w}_{\mathbf{c}_{1}} & \ldots & \mathbf{w}_{\mathbf{c}_{\mathbf{i}}} & \ldots & \mathbf{w}_{\mathbf{c}_{\mathrm{Nc}}}\end{array}\right\}$ é o vetor dos deslocamentos nos cantos,

- $\underset{\sim}{\mathbf{R}_{\mathbf{c}}^{\mathbf{T}}}=\left\{\begin{array}{lllll}\mathbf{R}_{\mathbf{c}_{1}} & \ldots & \mathbf{R}_{\mathbf{c}_{\mathbf{i}}} & \ldots & \mathbf{R}_{\mathbf{c}_{\mathrm{Nc}}}\end{array}\right\}$ é o vetor das reações de canto,

- $\hat{H}(\mathbf{Q}), \mathbf{G}(\mathbf{Q})$, são vetores de dimensão 1 x $2 \mathrm{~N}_{\mathrm{n}}$,

- $\mathbf{H}_{\mathbf{c}}(\mathbf{Q})$ e $\mathbf{G}_{\mathbf{c}}(\mathbf{Q})$ são vetores de dimensão 1 x N

- $\mathbf{N}_{\mathbf{n}}$, o número de nós do contorno da placa,

- $\mathbf{N}_{\mathrm{c}}, \mathrm{o}$ número de cantos.

Deve-se observar que o deslocamento $\mathbf{w}(\mathbf{Q})$ pode ser escrito em função dos deslocamentos nodais $\mathbf{U}_{\mathbf{j}}^{\mathbf{N}}$ do elemento ao qual pertence. Considerando-se que para todo canto, define-se três pontos nodais (2 nós duplos e um nó de canto), o ponto do contorno $\mathbf{Q}$ terá angulosidade somente se for um nó de canto. Assim, se o nó não for duplo e não for um nó de canto, tem-se que somar $\mathbf{K}(\mathbf{Q})=\mathbf{0 . 5}$ ao termo $\hat{\mathbf{H}}(\mathbf{Q}, \mathbf{2} * \mathbf{Q}-\mathbf{1})$ do sistema de equações, que é o termo referente ao deslocamento $\mathbf{w}$ do nó $\mathbf{Q}$, sendo $\mathbf{Q}$ o número do nó no contorno. No caso de $\mathbf{Q}$ ser um nó duplo, o mesmo é levado para dentro do elemento, como é mostrado no item (4.2.2) e o deslocamento do mesmo é escrito em função dos valores nodais do elemento, como está indicado em (4.12). Nesse caso, tem-se também que $\mathbf{K}(\mathbf{Q})=\mathbf{0 . 5}$ e portanto, deve-se somar $\mathbf{0 . 5} \phi_{\mathbf{i}}$ ao termo $\hat{\mathbf{H}}\left(\mathbf{Q}, \mathbf{2} * \mathbf{N} \mathbf{O}_{\mathbf{i}}-\mathbf{1}\right)$, que é o termo referente ao deslocamento $\mathbf{w}$ do nó $\mathbf{i}$, onde $\mathrm{i}=1,2,3$; $\mathbf{N o}_{\mathbf{i}}$ é o nó $\mathbf{i}$ do elemento ao qual $\mathbf{Q}$ pertence (ver figura 4.5) e $\phi_{\mathrm{i}}$ são as funções de forma dadas por (4.10). Logo a equação (4.23) fica: 


$$
\underset{\sim}{\mathbf{H}}(\mathbf{Q}) \underset{\sim}{\mathbf{U}}+\underset{\sim}{\mathbf{H}_{\mathbf{c}}}(\mathbf{Q}) \mathbf{w}_{\sim}^{\mathbf{c}}=\underset{\sim}{\mathbf{G}}(\mathbf{Q}) \underset{\sim}{\mathbf{P}}+\underset{\sim}{\mathbf{G}_{\mathbf{c}}}(\mathbf{Q}) \underset{\sim}{\mathbf{R}_{\mathbf{c}}}+\mathbf{T}(\mathbf{Q})
$$

No caso do ponto de colocação $\mathbf{Q}$ ser um nó de canto, a equação de deslocamento é dada por:

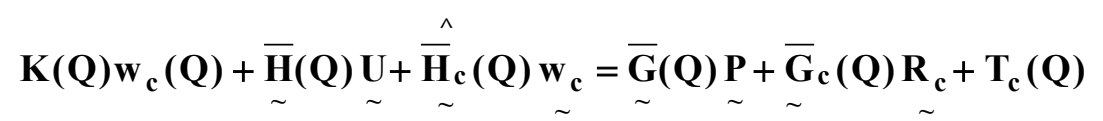

onde os vetores $\overline{\mathbf{H}}(\mathbf{Q}), \underset{\sim}{\hat{\mathbf{H}}_{\mathbf{c}}}(\mathbf{Q}), \underset{\sim}{\overline{\mathbf{G}}}(\mathbf{Q})$ e $\bar{\sim}_{\mathbf{c}}(\mathbf{Q})$ e o coeficiente $\mathbf{T}_{\mathbf{c}}(\mathbf{Q})$ são calculados, respectivamente, de maneira análoga a $\hat{H}(\mathbf{Q}), \mathbf{H}_{\mathbf{c}}(\mathbf{Q}), \mathbf{G}(\mathbf{Q}), \mathbf{G}_{\mathbf{c}}(\mathbf{Q})$ e $\mathbf{T}(\mathbf{Q})$, indicados na equação (4.23).

Nesse caso também, pode-se somar a constante $\mathbf{K}(\mathbf{Q})=\boldsymbol{\beta}_{\mathbf{c}} / \mathbf{2} \pi$ ao coeficiente $\hat{\overline{\mathbf{H}}}_{\mathbf{c}}(\mathbf{Q}, \mathbf{Q})$ da matriz $\hat{\sim}_{\mathbf{c}}(\mathbf{Q})$, que é o coeficiente que multiplica o deslocamento $\mathbf{w}_{\mathbf{c}}$ do canto considerado. Portanto, a equação (4.25) fica:

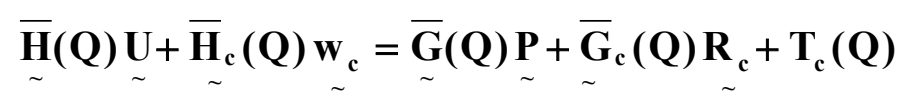

\subsection{Sistema de Equações}

Têm-se duas incógnitas nodais em cada ponto do contorno e uma em cada canto, portanto é necessário escrever duas equações para cada nó do contorno e uma para cada canto, para que seja possível se obter a solução do problema. Uma opção, é escrever, para cada nó do contorno, a equação algébrica do deslocamento $\mathbf{w}$, dada por (4.24) e a equação algébrica do deslocamento $\partial \mathbf{w} / \partial \mathbf{m}$, que pode ser obtida, derivando-se a equação (3.67). Contudo, nesse trabalho será usada somente a equação do deslocamento transversal w.

\subsubsection{Pontos de Colocação}

O sistema de equações poderá ser obtido de duas maneiras (ver figura 4.7): no caso a será escrito a equação de $\mathbf{w}$ em cada ponto do contorno $\mathbf{Q}$ e em seu respectivo ponto externo 
$\mathbf{A}$ e no caso $\mathbf{b}$, a mesma será escrita em dois pontos externos $\mathbf{A}_{\mathbf{1}} \mathbf{e} \mathbf{A}_{\mathbf{2}}$. A integração numérica, em ambos os casos, será feita usando a técnica de sub-elementos (ver item 4.7.2). Assim, para cada nó simples ou duplo do contorno, tem-se dois pontos de colocação e para cada nó de canto, tem-se um ponto de colocação. Em cada canto são definidos três pontos nodais (2 nós duplos e um nó de canto), o que resulta em 5 pontos de colocação, como está indicado na figura (4.7).
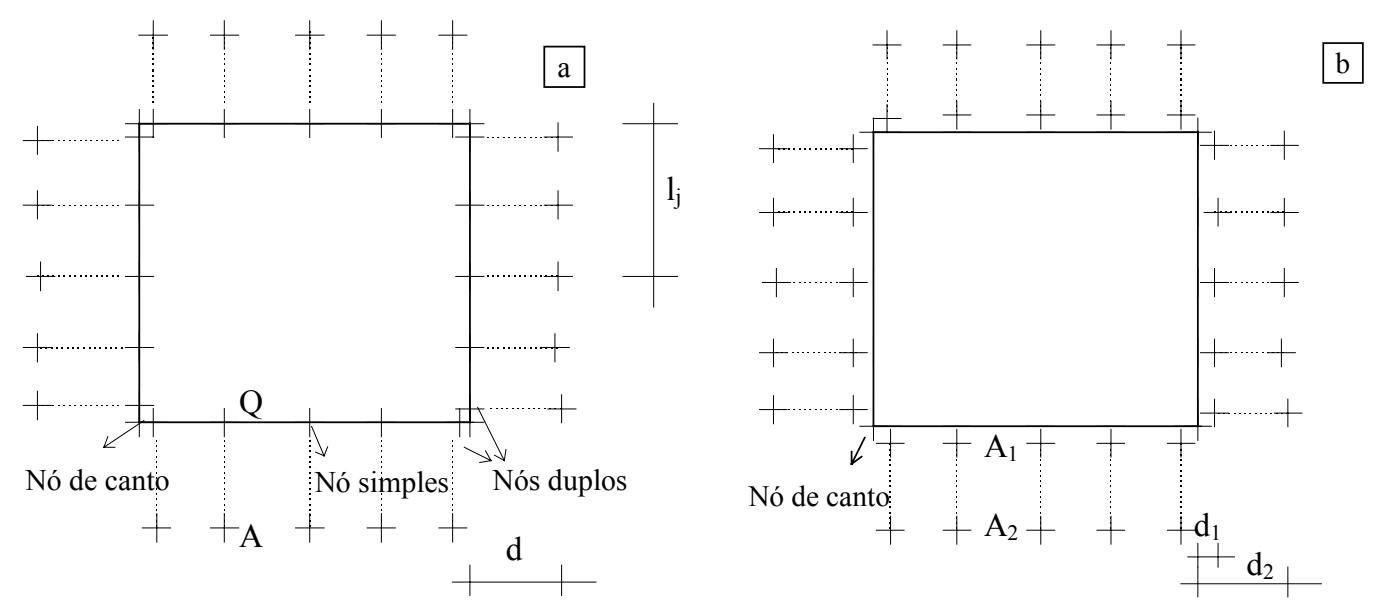

FIGURA 4.7 - Pontos de Colocação

onde: $\quad \mathbf{d}_{\mathbf{i}}=\mathbf{a}_{\mathbf{i}} \mathbf{l}_{\mathbf{m}}$

$\mathbf{I}_{\mathrm{m}}$ é a média dos comprimentos dos elementos concorrentes no nó, ou, se o nó for interno ao elemento, é igual ao comprimento do mesmo,

$\mathbf{0 . 0 0 0 1} \leq \mathbf{a}_{\mathbf{i}} \leq \mathbf{1 . 5}$ para evitar problemas numéricos,

$\mathbf{l}_{\mathbf{j}}$ é o comprimento do elemento $\mathbf{j}$.

Para se obter a solução não-linear do problema (ver capítulo 7) ou a solução linear considerando-se momentos iniciais (ver capítulo 5), é necessário que o domínio seja discretizado em células, nas quais o campo de momentos iniciais será aproximado. Como nesse trabalho a integração numérica das células não foi feita considerando-se a técnica de sub-elementos, o limite inferior de $\mathbf{a}_{\mathbf{i}}$ (equação 4.27) deve ser 0.1.

Além dos dois esquemas apresentados na figura (4.7), foi ainda testado um terceiro, onde considerava-se dois pontos de colocação no contorno, como está indicado na figura (4.8.a), onde está representada uma discretização com 20 nós no contorno. 


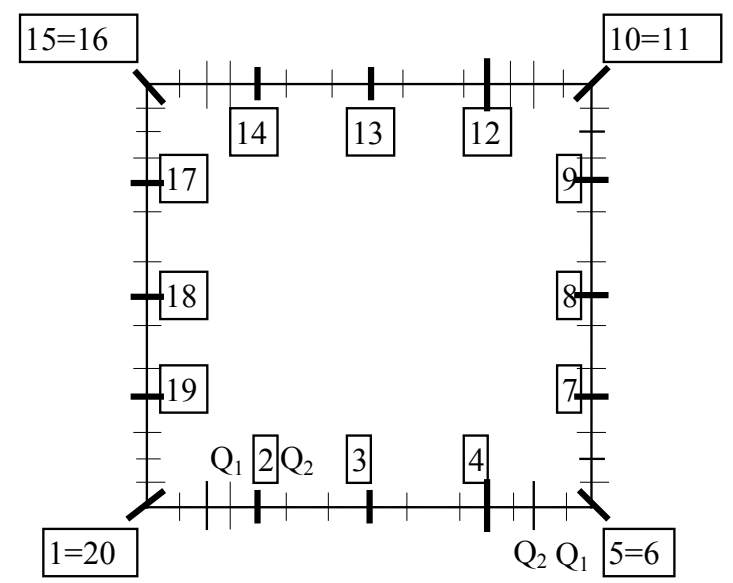

FIGURA 4.8 - Dois Pontos de Colocação no Contorno

Na figura (4.8), os nós 1 e 20, 5 e6, 10 e 11 e 15 e 16 são nós duplos. Para cada nó do contorno definiram-se dois pontos de colocação $\mathbf{Q}_{1}$ e $\mathbf{Q}_{2}$, no contorno, como está indicado para o nó simples 2 e o nó duplo 5. Porém, neste caso, constatou-se que o sistema se torna singular devido à dependência dos valores de contorno. Para que não ocorra singularidade no sistema, é necessário que pelo menos um ponto de colocação seja definido fora do contorno.

\subsubsection{Equação de Deslocamento para um Ponto Externo}

Ao escrever a equação (3.19) para um ponto externo, o seu primeiro termo será nulo, devido à propriedade da função delta de Dirac, dada por (3.6), que implica em:

$$
\int_{\Omega} \delta(\mathbf{A}, \mathbf{P}) \mathbf{w}(\mathbf{P}) \mathbf{d} \Omega(\mathbf{P})=\mathbf{0}
$$

pois, em $\Omega$ tem-se que $\delta(\mathbf{A}, \mathbf{P})=\mathbf{0}$.

Assim, a equação matricial do deslocamento para um ponto externo, é dada por:

$$
\begin{aligned}
& \int_{\Gamma} \mathbf{p}_{\sim}^{*}(\mathbf{A}, \mathbf{P}) \underset{\sim}{\mathbf{u}}(\mathbf{P}) \mathrm{d} \Gamma(\mathbf{P})+\sum_{\mathrm{i}=1}^{\mathrm{N}_{\mathrm{c}}} \mathbf{R}_{\mathrm{ci}}^{*}(\mathbf{A}, \mathbf{P}) \mathbf{w}_{\mathrm{ci}}(\mathbf{P})=\int_{\Gamma}^{\underset{\sim}{\mathbf{u}^{*}}} \underset{\sim}{*}(\mathbf{A}, \mathbf{P}) \underset{\sim}{\mathbf{p}}(\mathbf{P}) \mathrm{d} \Gamma(\mathbf{P})+ \\
& +\sum_{\mathrm{i}=1}^{\mathbf{N}_{\mathrm{c}}} \mathbf{R}_{\mathrm{ci}}(\mathbf{P}) \mathbf{w}_{\mathrm{ci}}^{*}(\mathbf{A}, \mathbf{P})+\int_{\Omega_{\mathrm{g}}} \mathbf{g}(\mathbf{p}) \mathbf{w}^{*}(\mathbf{A}, \mathbf{p}) \mathbf{d} \Omega_{\mathrm{g}}(\mathbf{p})
\end{aligned}
$$


onde todos os termos são análogos aos da equação (4.13).

Semelhante ao que foi feito no item (4.2.3), discretizando-se o contorno em $\mathbf{N}_{\mathbf{e}}$ elementos e somando-se as influências de todos os elementos, pode-se escrever a equação (4.29) matricialmente na seguinte forma:

$$
\underset{\sim}{\mathbf{H}}(\mathrm{A}) \underset{\sim}{\mathbf{U}}+\mathbf{H}_{\mathbf{c}}(\mathrm{A}) \mathbf{w}_{\mathbf{c}}=\underset{\sim}{\mathbf{G}}(\mathrm{A}) \underset{\sim}{\mathbf{P}}+\mathbf{G}_{\mathbf{c}}(\mathrm{A}) \mathbf{R}_{\mathrm{c}}+\underset{\sim}{\mathbf{T}}(\mathrm{A})
$$

onde os vetores e matrizes são análogos aos da equação (4.24), considerando-se $\mathbf{K}(\mathbf{A})=\mathbf{0}$.

\subsubsection{Obtenção das Incógnitas do Contorno e dos Cantos}

A equação (4.24) de deslocamento de um ponto $\mathbf{Q}$ do contorno pode ser escrita como:

$$
\left[\underset{\sim}{\mathbf{H}}(\mathbf{Q}) \quad \underset{\sim}{\mathbf{H}_{\mathbf{c}}}(\mathbf{Q})\right]\left\{\begin{array}{c}
\underset{\sim}{\mathbf{U}} \\
\underset{\sim}{\mathbf{w}}
\end{array}\right\}=\left[\underset{\sim}{\mathbf{G}(\mathbf{Q})} \quad \underset{\sim}{\mathbf{G}_{\mathbf{c}}(\mathbf{Q})}\right]\left[\begin{array}{c}
\underset{\sim}{\mathbf{P}} \\
\underset{\sim}{\mathbf{R}_{\mathbf{c}}}
\end{array}\right\}+\underset{\sim}{\mathbf{T}}(\mathbf{Q})
$$

De maneira análoga, escreve-se a equação (4.30) do deslocamento w para um ponto externo A. Assim, escrevendo-se as duas equações de deslocamento, correspondentes a um determinado ponto do contorno, em uma só equação matricial, obtém-se para o caso a do item (4.3.1), a seguinte equação:

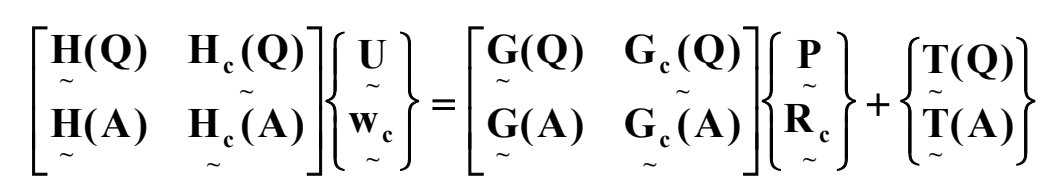

no caso $\mathbf{b}$, tem-se:

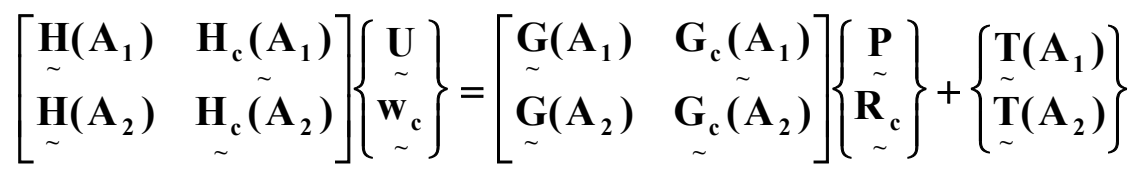

As equações (4.32) e (4.33) podem ser escritas de maneira simplificada da seguinte forma: 


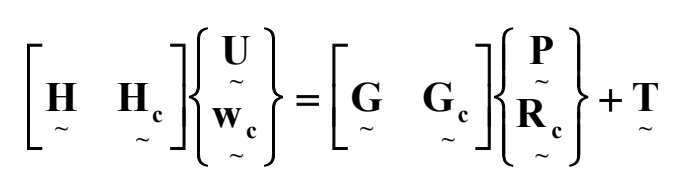

onde $\mathbf{H}$ e $\mathbf{G}$ são matrizes de dimensão $\left(2 \times 2 \mathrm{~N}_{\mathrm{n}}\right), \mathbf{H}_{\mathbf{c}}$ e $\mathbf{G}_{\mathbf{c}}$ são matrizes de dimensão $\left(2 \mathrm{xN}_{\mathrm{c}}\right)$ e o vetor $\mathbf{T}$ possui dois coeficientes.

Para um nó de canto, a equação matricial (4.26) do deslocamento w, pode também ser escrita da mesma forma:

$$
\left[\begin{array}{cc}
\overline{\mathbf{H}} & \overline{\mathbf{H}}_{\mathbf{c}}
\end{array}\right]\left\{\begin{array}{c}
\underset{\sim}{\mathbf{U}} \\
\underset{\sim}{\mathbf{w}}
\end{array}\right\}=\left[\begin{array}{cc}
\overline{\mathbf{G}} & \overline{\mathbf{G}}_{\mathbf{c}}
\end{array}\right]\left\{\begin{array}{c}
\underset{\sim}{\mathbf{P}} \\
\underset{\sim}{\mathbf{R}_{\mathbf{c}}}
\end{array}\right\}+\underset{\sim}{\mathbf{T}_{\mathbf{c}}}
$$

Portanto, escrevendo-se a equação (4.34) para todos os nós simples e duplos do contorno e a equação (4.35) para cada canto, obtém-se o seguinte sistema de equações:

$$
\left[\begin{array}{cc}
\underset{\sim}{\mathbf{H}} & \mathbf{H}_{\mathbf{c}} \\
\underset{\sim}{\mathbf{H}} & \tilde{\overline{\mathbf{H}}}_{\mathbf{c}}
\end{array}\right]\left\{\begin{array}{c}
\underset{\sim}{\mathbf{U}} \\
\underset{\mathbf{w}_{\mathbf{c}}}{\sim}
\end{array}\right\}=\left[\begin{array}{cc}
\underset{\sim}{\mathbf{G}} & \mathbf{G}_{\mathbf{c}} \\
\underset{\sim}{\mathbf{G}} & \tilde{\overline{\mathbf{G}}}_{\mathbf{c}}
\end{array}\right]\left\{\begin{array}{c}
\mathbf{P} \\
\underset{\sim}{\mathbf{R}_{\mathbf{c}}}
\end{array}\right\}+\left\{\begin{array}{c}
\mathbf{T} \\
\tilde{\sim} \\
\underset{\sim}{\mathbf{T}}
\end{array}\right\}
$$

onde:

- $\quad$ H e $\mathbf{G}$ são submatrizes de dimensão $2 \mathrm{~N}_{\mathrm{n}} \times 2 \mathrm{~N}_{\mathrm{n}}$, compostas pelos vetores $\mathbf{H}$ e $\mathbf{G}$, dados em (4.34). São correspondentes às variáveis nodais;

- $\mathbf{H}_{\mathbf{c}}$ e $\mathbf{G}_{\mathbf{c}}$ são submatrizes de dimensão $2 \mathrm{~N}_{\mathrm{n}} \times \mathrm{N}_{\mathrm{c}}$, compostas pelos vetores $\mathbf{H}_{\mathbf{c}}$ e $\mathbf{G}_{\mathbf{c}}$, dados em (4.34). São correspondentes às variáveis dos cantos;

- $\quad$ T é o subvetor de dimensão $2 \mathrm{~N}_{\mathrm{n}} \times 1$, constituído pelos vetores $\mathbf{T}$, dado em (4.34);

- $\underset{\sim}{\overline{\mathbf{H}}}, \underset{\sim}{\mathbf{G}}, \overline{\mathbf{H}}_{\mathbf{c}}$ e $\overline{\mathbf{G}}_{\mathbf{c}}$ são submatrizes cujos coeficientes são semelhantes, respectivamente, aos de $\underset{\sim}{\mathbf{H}}, \underset{\sim}{\mathbf{G}}, \mathbf{H}_{\mathbf{c}}$ e $\mathbf{G}_{\mathbf{c}}$, e são compostas pelos vetores indicados na equação (4.35), associada a um canto da placa. $\underset{\sim}{\overline{\mathbf{H}}}$ e $\underset{\sim}{\overline{\mathbf{G}}}$ têm dimensão $\mathrm{N}_{\mathrm{c}} \times \mathrm{N}_{\mathrm{n}}$ e $\overline{\mathbf{H}}_{\mathbf{c}}$ e $\overline{\mathbf{G}}_{\mathbf{c}}$ são quadradas de ordem $\mathrm{N}_{\mathrm{c}}$; 
- $\mathbf{T}_{\mathbf{c}}$ é o subvetor de dimensão $\mathbf{N}_{\mathbf{c}} \times 1$, cujos coeficientes são semelhantes aos de $\mathbf{T}$, e que são obtidos quando se escreve a equação (4.35) para todos os cantos da placa.

De maneira simplificada, o sistema (4.36) pode ser escrito como:

$$
\left[\begin{array}{cc}
\overline{\mathbf{H}}_{\mathrm{Nn}}^{*} & \overline{\mathbf{H}}_{\mathbf{C}}^{*}
\end{array}\right]\left\{\begin{array}{c}
\underset{\sim}{\mathbf{U}} \\
\underset{\sim}{\mathbf{w}}
\end{array}\right\}=\left[\begin{array}{cc}
\overline{\mathbf{G}}_{\mathrm{Nn}}^{*} & \overline{\mathbf{G}}_{\mathrm{C}}^{*}
\end{array}\right]\left\{\begin{array}{c}
\underset{\sim}{\mathbf{P}} \\
\underset{\sim}{\mathbf{R}}
\end{array}\right\}+\bar{\sim}^{*}
$$

ou ainda:

$$
\bar{\sim}^{*} \underset{\sim}{\mathbf{U}}=\bar{\sim}^{*} \underset{\sim}{\mathbf{P}}+\bar{\sim}^{*}
$$

Após a imposição das condições de contorno, o sistema (4.38) pode ser resolvido, isolando-se todas as incógnitas no vetor $\overline{\mathbf{U}}$, trocando-se as respectivas colunas das matrizes $\overline{\mathbf{H}}^{*}$ e $\overline{\mathbf{G}}^{*}$, fazendo-se a multiplicação dessa nova matriz $\overline{\mathbf{G}}^{*}$ com o vetor $\overline{\mathbf{P}}$, que agora contém os valores prescritos, e somando esse último com o vetor $\overline{\mathbf{T}}^{*}$. Assim, obtém-se um sistema da forma:

$$
\underset{\sim}{\mathbf{A}} \underset{\sim}{\mathbf{X}}=\underset{\sim}{\mathbf{B}}
$$

onde: $\mathbf{X}$ é o vetor solução, composto pelos deslocamentos e esforços incógnitos nos nós do contorno e cantos da placa e $\underset{\sim}{\mathbf{B}}=\bar{\sim}_{\sim}^{*} \underset{\sim}{\mathbf{P}}+\bar{\sim}^{*}$.

\subsection{Propriedades da Matriz H}

A matriz $\bar{\sim}^{*}$ possui propriedades, que podem ser estabelecidas através de configurações de equilíbrio particulares de uma placa.

Assim, admitindo-se um carregamento transversal nulo, os vetores dos esforços nodais $\underset{\sim}{\mathbf{P}}$, das reações de canto $\underset{\sim}{\mathbf{R}_{\mathfrak{c}}}$ e das integrais sobre a região carregada ${\underset{\sim}{\mathbf{T}}}^{*}$, do sistema de equações (4.37), são nulos, podendo-se escrever:

$$
\overline{\mathbf{H}}_{\sim}^{*} \cdot \underset{\sim}{\mathbf{U}}+\overline{\mathbf{H}}_{\mathrm{C}}^{*} \cdot \underline{\sim}_{\mathrm{C}}=\mathbf{0}
$$


Este sistema de equação admite soluções não triviais, correspondentes aos movimentos de corpo rígido, isto é, deslocamento transversal e rotações em torno de eixos arbitrários, PAIVA (1987).

Assim, considerando-se um deslocamento transversal de corpo rígido $\mathbf{w}_{\mathbf{0}}$ na placa (ver figura 4.9), o vetor de deslocamento $\mathbf{U}$ da equação (4.40) fica:

$$
\underset{\sim}{\mathbf{U}^{\mathrm{T}}}=\left\{\begin{array}{llllllll}
w_{0} & 0 & w_{0} & 0 & \ldots & \ldots & w_{0} & 0
\end{array}\right\}
$$

ou considerando-se apenas $\mathbf{w}_{\mathbf{c}}$ :

$$
\underset{\mathrm{c}}{w_{\mathrm{c}}^{\mathrm{T}}}=\left\{\begin{array}{lllll}
w_{0} & w_{0} & w_{0} & \ldots & w_{0}
\end{array}\right\}
$$

Substituindo-se (4.41) e (4.42) em (4.40), obtém-se a propriedade, relativa ao movimento de corpo rígido na direção do deslocamento transversal w, e portanto, que envolve os elementos das colunas ímpares de $\overline{\mathbf{H}}_{\text {Nn }}^{*}$, para qualquer linha i da mesma. Assim, pode-se escrever:

$$
\sum_{j=1}^{N n} \overline{\mathbf{h}}_{i, 2 j-1}^{*}+\sum_{j=1}^{N c} \overline{\mathbf{h}}_{\mathbf{C}_{i, j}}^{*}=0
$$

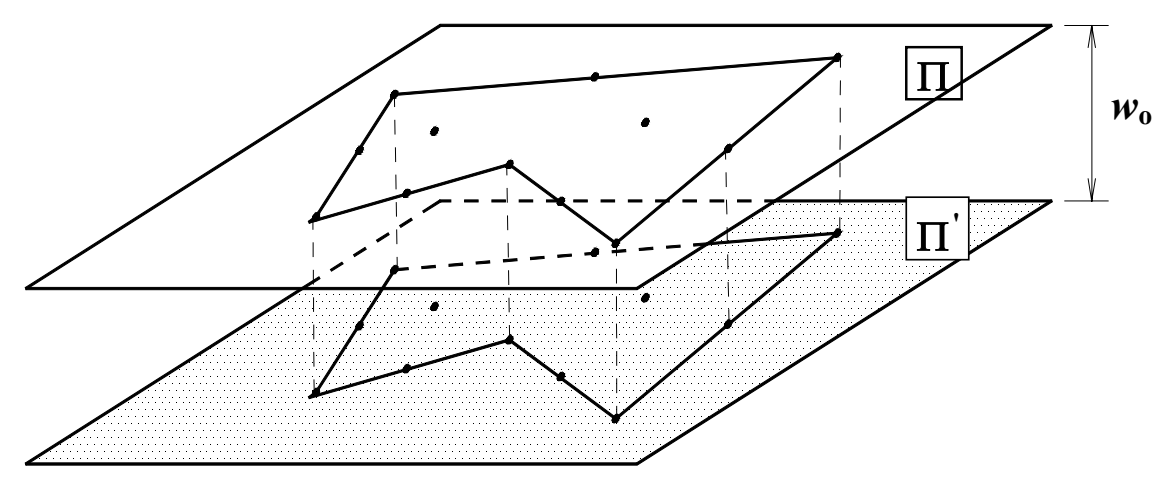

FIGURA 4.9 - Movimento de Corpo Rígido - Translação 


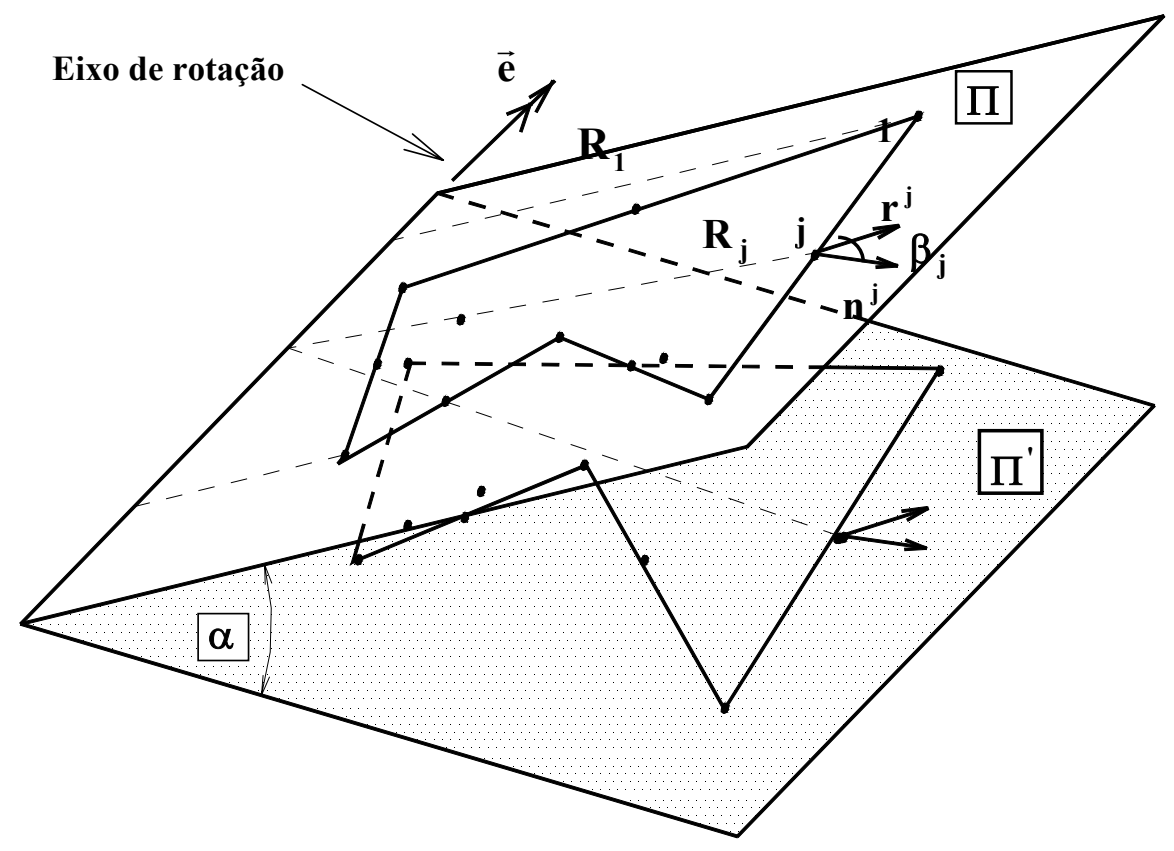

FIGURA 4.10 - Movimento de Corpo Rígido - Rotação.

onde $\mathbf{R}_{\mathbf{j}}$ é o valor de $\mathbf{r}$ para um nó genérico $\mathbf{j}$ e representa a distância entre e o nó $\mathbf{j}$ e o eixo de rotação; $\mathbf{n}^{\mathbf{j}}$ e $\mathbf{r}^{\mathbf{j}}$ indicam, respectivamente, as direções da normal ao contorno e de $\mathbf{r}$ no nó j. 
Considerando-se agora uma rotação de corpo rígido $\alpha$ (ver figura 4.10), no sentido indicado pelo eixo arbitrário $\overrightarrow{\mathbf{e}}$, os vetores de deslocamento ficam:

$$
\begin{aligned}
& \underset{\sim}{\mathbf{U}^{\mathrm{T}}}=\alpha \cdot\left\{\begin{array}{llllllll}
\mathbf{R}_{1} & \cos \beta_{1} & \ldots & \mathbf{R}_{\mathrm{j}} & \cos \beta_{\mathrm{j}} & \ldots & \mathbf{R}_{\mathrm{Nn}} & \cos \beta_{\mathrm{Nn}}
\end{array}\right\} \\
& \underset{\sim}{w_{c}^{T}}=\alpha \cdot\left\{\begin{array}{lllll}
\mathbf{R}_{\mathrm{c} 1} & \mathbf{R}_{\mathrm{c} 2} & \mathbf{R}_{\mathrm{c} 3} & \ldots & \mathbf{R}_{\mathrm{cNc}}
\end{array}\right\}
\end{aligned}
$$

onde $\cos \beta_{\mathbf{j}}=\mathbf{n}_{\mathbf{k}}^{\mathbf{j}} \cdot \mathbf{r}_{\mathbf{k}}^{\mathbf{j}}$, sendo que $\mathbf{n}_{\mathbf{k}}^{\mathbf{j}}$ e $\mathbf{r}_{\mathbf{k}}^{\mathbf{j}}$ são os cossenos diretores de $\mathbf{n}^{\mathbf{j}}$ e $\mathbf{r}^{\mathbf{j}}$, respectivamente.

Substituindo-se (4.44) e (4.45) em (4.40), chega-se a segunda propriedade:

$$
\sum_{j=1}^{N n}\left(\overline{\mathbf{h}}_{i, 2 j-1}^{*} \mathbf{R}_{j}+\overline{\mathbf{h}}_{i, 2 j}^{*} \cos \beta_{j}\right)+\sum_{j=1}^{N c} \overline{\mathbf{h}}_{\mathrm{C}_{i, j}}^{*} \mathbf{R}_{\mathrm{C}_{\mathrm{j}}}=\mathbf{0}
$$

Estas propriedades podem ser usadas para verificação das matrizes $\mathbf{H}$ e $\mathbf{H}_{\mathbf{c}}$.

\subsection{Deslocamentos e Esforços para Pontos Internos}

Após a determinação dos deslocamentos e esforços no contorno da placa, é necessária a obtenção destes valores para os pontos do seu interior. O deslocamento w(q) de um ponto interno é dado pela equação (3.19). Assim, escrevendo-se a mesma para $\mathbf{N}_{\mathbf{i}}$ pontos internos e fazendo-se a discretização do contorno e a aproximação das variáveis, de forma semelhante ao que foi feito para obter a equação (4.23), os deslocamentos $\mathbf{w}$ dos pontos internos são dados pela equação matricial:

$$
\underset{\sim}{\mathbf{w}}(\mathbf{q})+\underset{\sim}{\mathbf{H}^{*}}(\mathbf{q}) \underset{\sim}{\mathbf{U}}+\underset{\sim}{\mathbf{H}_{\mathbf{c}}^{*}}(\mathbf{q}) \mathbf{\sim}_{\mathbf{c}}^{\mathbf{w}}={\underset{\sim}{\mathbf{G}}}^{*}(\mathbf{q}) \underset{\sim}{\mathbf{P}}+\underset{\sim}{\mathbf{G}_{\mathbf{c}}^{*}}(\mathbf{q}) \mathbf{R}_{\mathbf{c}}^{\mathbf{R}}+\underset{\sim}{\mathbf{T}^{*}}(\mathbf{q})
$$

onde:

- o vetor $\mathbf{w}(\mathbf{q})$ contém os valores dos deslocamentos $\mathbf{w}$ dos pontos internos e tem dimensão $\mathrm{N}_{\mathrm{i}} \times 1$, 
- os vetores $\{\mathbf{U}\},\left\{\mathbf{w}_{\mathbf{c}}\right\},\{\mathbf{P}\}$ e $\left\{\mathbf{R}_{\mathbf{c}}\right\}$ são os vetores de deslocamentos e esforços dos nós e cantos do contorno, já calculados,

- as matrizes [ $\left.\mathbf{H}^{*}\right]$ e $\left[\mathbf{G}^{*}\right]$ têm dimensões $\mathrm{N}_{\mathrm{i}}$ x $2 \mathrm{~N}_{\mathrm{n}}$ e seus coeficientes são obtidos por (4.19) e (4.20),respectivamente,

- as matrizes $\left[\mathbf{H}_{\mathbf{c}}^{*}\right]$ e $\left[\mathbf{G}_{\mathbf{c}}^{*}\right]$, têm dimensões $\mathrm{N}_{\mathrm{i}} \times \mathrm{N}_{\mathrm{c}}$ e são semelhantes às da equação $(4.23)$

- o vetor $\mathbf{T}^{*}$ (q) tem dimensão $\mathrm{N}_{\mathrm{i}} \mathrm{x}$ 1, e é semelhante ao da equação (4.23).

O cálculo dos momentos $\mathbf{M}_{\mathrm{ij}}$ para um ponto interno é feito a partir da equação (2.9), na qual as curvaturas $\mathbf{w},_{\mathbf{i j}}$ são obtidas derivando-se a equação (3.19) do deslocamento $\mathbf{w}(\mathbf{q})$ em relação às direções $\mathbf{x}_{1}$ e $\mathbf{x}_{2}$. Assim, a equação das curvaturas, na forma matricial, é dada por:

$$
\begin{aligned}
& \underset{\sim}{\mathbf{w}},_{\mathrm{ij}}(\mathbf{q})+\int_{\Gamma} \mathbf{p}_{\sim}^{*}(\mathbf{q}, \mathbf{P}) \underset{\sim}{\mathbf{u}}(\mathbf{P}) \mathbf{d} \Gamma(\mathbf{P})+\sum_{\mathrm{i}=1}^{\mathbf{N}_{\mathrm{c}}} \mathbf{p}_{\sim}^{*}{ }_{\sim}^{*}(\mathbf{q}, \mathbf{P}) \mathbf{w}_{\mathrm{ci}}(\mathbf{P})=
\end{aligned}
$$

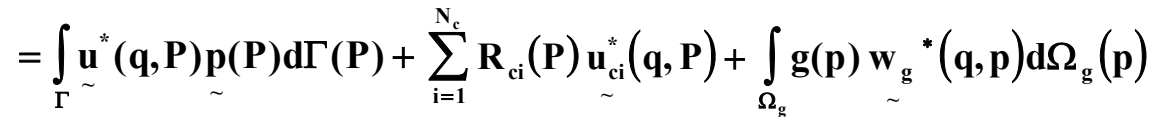

onde: $\quad \mathbf{w},,_{\mathrm{ij}}{ }^{\mathrm{T}}(\mathbf{q})=\left\{\begin{array}{lll}\frac{\partial^{2} \mathbf{w}(\mathbf{q})}{\partial \mathbf{x}_{1} \partial \mathbf{x}_{1}} & \frac{\partial^{2} \mathbf{w}(\mathbf{q})}{\partial \mathbf{x}_{1} \partial \mathbf{x}_{2}} & \frac{\partial^{2} \mathbf{w}(\mathbf{q})}{\partial \mathbf{x}_{2} \partial \mathbf{x}_{2}}\end{array}\right\}$

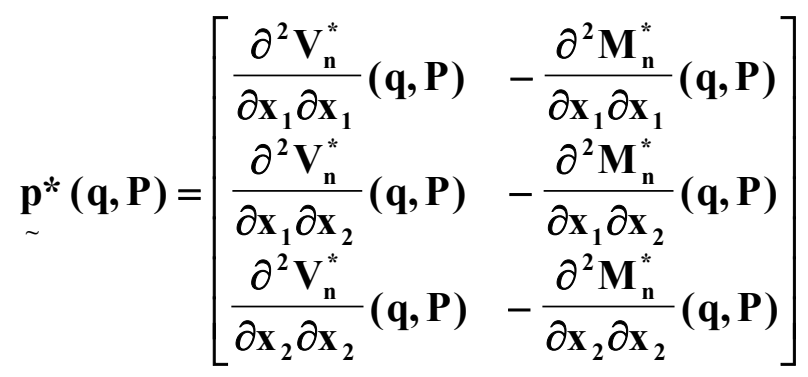

$$
\begin{aligned}
& \underset{\sim}{\mathbf{u}^{\mathrm{T}}}(\mathbf{P})=\left\{\mathbf{u}_{1}(\mathbf{P}) \quad \mathbf{u}_{2}(\mathbf{P}\}=\left\{\mathbf{w}(\mathbf{P}) \quad \frac{\partial \mathbf{w}}{\partial \mathbf{n}}(\mathbf{P})\right\}\right. \\
& \underset{\sim}{\mathbf{p}_{\mathrm{ci}}^{* \mathrm{~T}}}(\mathbf{q}, \mathbf{P})=\left\{\begin{array}{lll}
\frac{\partial^{2} \mathbf{R}_{\mathrm{ci}}^{*}(\mathbf{q}, \mathbf{P})}{\partial \mathbf{x}_{1} \partial \mathbf{x}_{1}} & \frac{\partial^{2} \mathbf{R}_{\mathrm{ci}}^{*}(\mathbf{q}, \mathbf{P})}{\partial \mathbf{x}_{1} \partial \mathbf{x}_{2}} & \frac{\partial^{2} \mathbf{R}_{\mathrm{ci}}^{*}(\mathbf{q}, \mathbf{P})}{\partial \mathbf{x}_{2} \partial \mathbf{x}_{2}}
\end{array}\right\}
\end{aligned}
$$




$$
\begin{aligned}
& \underset{\sim}{\mathbf{u}}(\mathbf{q}, \mathbf{P})=\left[\begin{array}{cc}
\frac{\partial^{2} \mathbf{w}^{*}}{\partial \mathbf{x}_{1} \partial \mathbf{x}_{1}}(\mathbf{q}, \mathbf{P}) & -\frac{\partial^{2}}{\partial \mathbf{x}_{1} \partial \mathbf{x}_{1}}\left(\frac{\partial^{2} \mathbf{w}^{*}}{\partial \mathbf{n}}(\mathbf{q}, \mathbf{P})\right) \\
\frac{\partial^{2} \mathbf{w}^{*}}{\partial \mathbf{x}_{1} \partial \mathbf{x}_{2}}(\mathbf{q}, \mathbf{P}) & -\frac{\partial^{2}}{\partial \mathbf{x}_{1} \partial \mathbf{x}_{2}}\left(\frac{\partial^{2} \mathbf{w}^{*}}{\partial \mathbf{n}}(\mathbf{q}, \mathbf{P})\right) \\
\frac{\partial^{2} \mathbf{w}^{*}}{\partial \mathbf{x}_{2} \partial \mathbf{x}_{2}}(\mathbf{q}, \mathbf{P}) & -\frac{\partial^{2}}{\partial \mathbf{x}_{2} \partial \mathbf{x}_{2}}\left(\frac{\partial^{2} \mathbf{w}^{*}}{\partial \mathbf{n}}(\mathbf{q}, \mathbf{P})\right)
\end{array}\right] \\
& {\underset{\sim}{p}}^{T}(P)=\left\{\mathbf{p}_{1}(P) \quad \mathbf{p}_{2}(P)\right\}=\left\{V_{n}(P) \quad M_{n}(P)\right\} \\
& \underset{\sim}{\mathbf{u}_{\mathrm{ci}}^{*} \mathrm{~T}}(\mathbf{q}, \mathbf{P})=\left\{\frac{\partial^{2} \mathbf{w}_{\mathrm{ci}}^{*}}{\partial \mathbf{x}_{1} \partial \mathbf{x}_{1}}(\mathbf{q}, \mathbf{P}) \frac{\partial^{2} \mathbf{w}_{\mathrm{ci}}^{*}}{\partial \mathbf{x}_{1} \partial \mathbf{x}_{2}}(\mathbf{q}, \mathbf{P}) \frac{\partial^{2} \mathbf{w}_{\mathrm{ci}}^{*}}{\partial \mathbf{x}_{2} \partial \mathbf{x}_{2}}(\mathbf{q}, \mathbf{P})\right\} \\
& \underset{\sim}{\mathbf{w}_{\mathbf{g}}^{*} \mathbf{T}}(\mathbf{q}, \mathbf{P})=\left\{\frac{\partial^{2} \mathbf{w}^{*}}{\partial \mathbf{x}_{1} \partial \mathbf{x}_{1}}(\mathbf{q}, \mathbf{P}) \frac{\partial^{2} \mathbf{w}^{*}}{\partial \mathbf{x}_{1} \partial \mathbf{x}_{2}}(\mathbf{q}, \mathbf{P}) \frac{\partial^{2} \mathbf{w}^{*}}{\partial \mathbf{x}_{2} \partial \mathbf{x}_{2}}(\mathbf{q}, \mathbf{P})\right\}
\end{aligned}
$$

onde as derivadas dos deslocamentos e esforços fundamentais estão indicadas no item (3.2.1).

Após a discretização do contorno e a aproximação das variáveis, as curvaturas em $\mathbf{N}_{\mathbf{i}}$ pontos internos são calculadas através da equação matricial:

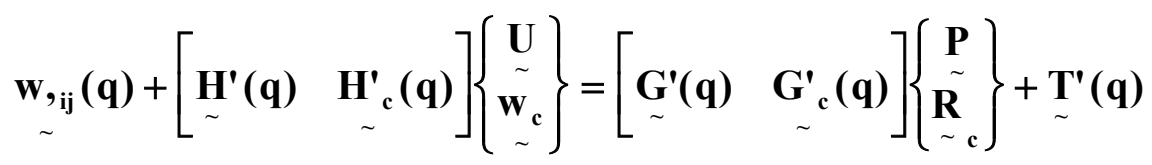

onde:

- $\mathbf{w},{ }_{\mathrm{ij}}(\mathbf{q})$ é o vetor que contém as curvaturas nos pontos internos,

- os vetores $\{\mathbf{U}\},\left\{\mathbf{w}_{\mathbf{c}}\right\},\{\mathbf{P}\}$ e $\left\{\mathbf{R}_{\mathbf{c}}\right\}$ são os vetores de deslocamentos e esforços dos nós e cantos do contorno, já calculados,

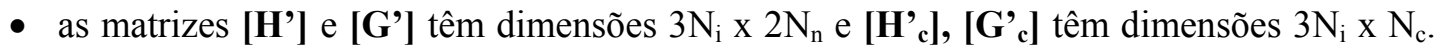
Os coeficientes de [H'] e [G'] são provenientes das integrais sobre os elementos do contorno discretizado expressas em (4.19) e (4.20), porém com $\mathbf{p}^{*}(\mathbf{q}, \mathbf{P}) \underset{\sim}{\text { e }} \underset{\sim}{\mathbf{u}}(\mathbf{q}, \mathbf{P})$ definidos em (4.50) e (4.53). 
Os momentos elásticos, nos pontos internos, são obtidos, substituindo-se os valores de $\mathbf{w},_{i j}$, dados por (4.57), na equação (2.9). Assim, pode-se escrever a equação dos momentos elásticos, em $\mathbf{N}_{\mathbf{i}}$ pontos internos, da seguinte maneira:

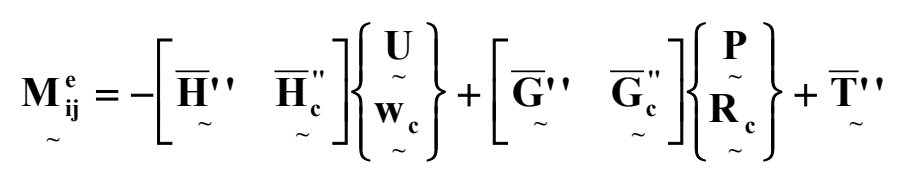

Ou de uma forma mais simplicada:

$$
\mathbf{M}_{\mathrm{ij}}^{\mathrm{e}}=-\left[\overline{\mathbf{H}}^{\prime}{ }^{\prime}\right]\{\mathbf{U}\}+\left[\overline{\mathbf{G}}^{\prime \prime}\right]\{\mathbf{P}\}+\left\{\overline{\mathbf{T}}^{\prime \prime}\right\}
$$

onde:

- $\left[\overline{\mathbf{H}}^{\prime}\right]$ e $\left[\overline{\mathbf{G}}^{\prime}\right.$ '], têm dimensão $3 \mathrm{~N}_{\mathrm{i}} \mathrm{x}\left(2 \mathrm{~N}_{\mathrm{n}}+\mathrm{N}_{\mathrm{c}}\right)$,

- $\{\mathbf{U}\}$ contêm os deslocamentos nos pontos do contorno e nos cantos,

- $\{\mathbf{P}\}$ contêm os esforços nos pontos do contorno e nos cantos.

Considerando-se a equação (2.9), os coeficientes de [ $\overline{\mathbf{H}}^{\prime}$ '] são dados por:

$$
\begin{aligned}
& \bar{H}^{\prime} \prime(3 I-2, k)=-D\left[v\left(H^{\prime}(3 I-2, k)+H^{\prime}(3 I, k)\right)+(1-v) H^{\prime}(3 I-2, k)\right] \\
& \bar{H}^{\prime} \prime(3 I-1, k)=-D\left[(1-v) H^{\prime}(3 I-1, k)\right] \\
& \bar{H}^{\prime} \prime(3 I, k)=-D\left[v\left(H^{\prime}(3 I-2, k)+H^{\prime}(3 I, k)\right)+(1-v) H^{\prime}(3 I, k)\right]
\end{aligned}
$$

onde I é o número do ponto interno, os coeficientes de $\mathbf{H}_{\sim}^{\prime}$ são dados pela equação (4.57) e k é o nó do contorno ou de canto.

Procedendo-se de maneira análoga ao que foi feito para $\overline{\mathbf{H}}^{\prime \prime}$, pode-se obter os coeficientes de $\overline{\mathbf{G}}_{\sim}^{\prime \prime}$.

A força cortante $\mathbf{Q}_{\mathbf{j}}$ é calculada a partir da equação (2.13), onde o valor de $\mathbf{w},_{\mathbf{k k j}}$, que é calculado derivando a equação (3.19) do deslocamento w, é dado pela equação matricial: 


$$
\begin{aligned}
& \underset{\sim}{\mathbf{w}_{\mathbf{k k j}}}(\mathbf{q})+\int_{\Gamma} \underset{\sim}{\mathbf{p}^{*}}(\mathbf{q}, \mathbf{P}) \underset{\sim}{\mathbf{u}}(\mathbf{P}) \mathrm{d} \Gamma(\mathbf{P})+\sum_{\mathrm{i}=1}^{\mathbf{N}_{\mathrm{c}}} \mathbf{p}_{\sim}^{*}{ }_{\mathrm{c}}^{*}(\mathbf{q}, \mathbf{P}) \mathbf{w}_{\mathrm{ci}}(\mathbf{P})=
\end{aligned}
$$

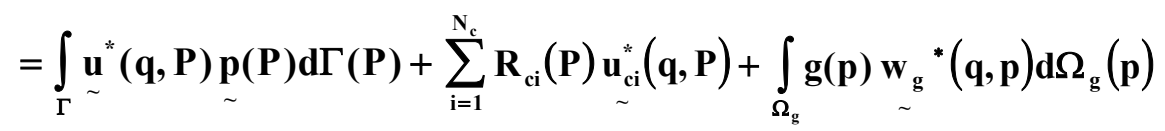

onde: $\quad \underset{\sim}{\mathbf{w}} \underset{\mathbf{k} \mathbf{k j}}{\mathbf{T}}(\mathbf{q})=\left\{\frac{\partial}{\partial \mathbf{x}_{1}}\left(\frac{\partial^{2} \mathbf{w}(\mathbf{q})}{\partial \mathbf{x}_{\mathbf{k}} \partial \mathbf{x}_{\mathbf{k}}}\right) \frac{\partial}{\partial \mathbf{x}_{2}}\left(\frac{\partial^{2} \mathbf{w}(\mathbf{q})}{\partial \mathbf{x}_{\mathbf{k}} \partial \mathbf{x}_{\mathbf{k}}}\right)\right\}$

$$
\begin{aligned}
& \underset{\sim}{\mathbf{p}^{*}}(\mathbf{q}, \mathbf{P})=\left[\begin{array}{cr}
\frac{\partial}{\partial \mathbf{x}_{1}}\left(\frac{\partial^{2} \mathbf{V}_{\mathbf{n}}^{*}}{\partial \mathbf{x}_{\mathbf{k}} \partial \mathbf{x}_{\mathbf{k}}}(\mathbf{q}, \mathbf{P})\right) & -\frac{\partial}{\partial \mathbf{x}_{1}}\left(\frac{\partial^{2} \mathbf{M}_{\mathbf{n}}^{*}}{\partial \mathbf{x}_{\mathbf{k}} \partial \mathbf{x}_{\mathbf{k}}}(\mathbf{q}, \mathbf{P})\right) \\
\frac{\partial}{\partial \mathbf{x}_{2}}\left(\frac{\partial^{2} \mathbf{V}_{\mathbf{n}}^{*}}{\partial \mathbf{x}_{\mathbf{k}} \partial \mathbf{x}_{\mathbf{k}}}(\mathbf{q}, \mathbf{P})\right) & -\frac{\partial}{\partial \mathbf{x}_{1}}\left(\frac{\partial^{2} \mathbf{M}_{\mathbf{n}}^{*}}{\partial \mathbf{x}_{\mathbf{k}} \partial \mathbf{x}_{\mathbf{k}}}(\mathbf{q}, \mathbf{P})\right)
\end{array}\right] \\
& \underset{\sim}{\mathbf{u}^{\mathrm{T}}}(\mathbf{P})=\left\{\mathbf{u}_{1}(\mathbf{P}) \quad \mathbf{u}_{2}(\mathbf{P})\right\}=\left\{\mathbf{w}(\mathbf{P}) \quad \frac{\partial \mathbf{w}}{\partial \mathbf{n}}(\mathbf{P})\right\} \\
& \underset{\sim}{\mathbf{p}_{\mathbf{c i}}^{*} \mathbf{T}}(\mathbf{q}, \mathbf{P})=\left\{\frac{\partial}{\partial \mathbf{x}_{1}}\left(\frac{\partial^{2} \mathbf{R}_{\mathbf{c i}}^{*}(\mathbf{q}, \mathbf{P})}{\partial \mathbf{x}_{\mathbf{k}} \partial \mathbf{x}_{\mathbf{k}}}\right) \quad \frac{\partial}{\partial \mathbf{x}_{2}}\left(\frac{\partial^{2} \mathbf{R}_{\mathrm{ci}}^{*}(\mathbf{q}, \mathbf{P})}{\partial \mathbf{x}_{\mathbf{k}} \partial \mathbf{x}_{\mathbf{k}}}\right)\right\} \\
& \underset{\sim}{\mathbf{u}^{*}}(\mathbf{q}, \mathbf{P})=\left[\begin{array}{cc}
\frac{\partial}{\partial \mathbf{x}_{1}}\left(\frac{\partial^{2} \mathbf{w}^{*}}{\partial \mathbf{x}_{\mathbf{k}} \partial \mathbf{x}_{\mathbf{k}}}(\mathbf{q}, \mathbf{P})\right) & -\frac{\partial}{\partial \mathbf{x}_{1}}\left(\frac{\partial^{2}}{\partial \mathbf{x}_{\mathbf{k}} \partial \mathbf{x}_{\mathbf{k}}}\left(\frac{\partial^{2} \mathbf{w}^{*}}{\partial \mathbf{n}}(\mathbf{q}, \mathbf{P})\right)\right) \\
\frac{\partial}{\partial \mathbf{x}_{2}}\left(\frac{\partial^{2} \mathbf{w}^{*}}{\partial \mathbf{x}_{\mathbf{k}} \partial \mathbf{x}_{k}}(\mathbf{q}, \mathbf{P})\right) & -\frac{\partial}{\partial \mathbf{x}_{2}}\left(\frac{\partial^{2}}{\partial \mathbf{x}_{\mathbf{k}} \partial \mathbf{x}_{\mathbf{k}}}\left(\frac{\partial^{2} \mathbf{w}^{*}}{\partial \mathbf{n}}(\mathbf{q}, \mathbf{P})\right)\right)
\end{array}\right] \\
& {\underset{\sim}{\mathbf{p}}}^{\mathrm{T}}(\mathbf{P})=\left\{\mathbf{p}_{1}(\mathbf{P}) \quad \mathbf{p}_{2}(\mathbf{P})\right\}=\left\{\mathbf{V}_{\mathrm{n}}(\mathbf{P}) \quad \mathbf{M}_{\mathrm{n}}(\mathbf{P})\right\} \\
& \underset{\sim}{\mathbf{u}_{\mathbf{c i}}^{* T}}(\mathbf{q}, \mathbf{P})=\left\{\frac{\partial}{\partial \mathbf{x}_{1}}\left(\frac{\partial^{2} \mathbf{w}_{\mathbf{c} i}^{*}}{\partial \mathbf{x}_{\mathbf{k}} \partial \mathbf{x}_{\mathbf{k}}}(\mathbf{q}, \mathbf{P})\right) \frac{\partial}{\partial \mathbf{x}_{2}}\left(\frac{\partial^{2} \mathbf{w}_{\mathbf{c i}}^{*}}{\partial \mathbf{x}_{\mathbf{k}} \partial \mathbf{x}_{\mathbf{k}}}(\mathbf{q}, \mathbf{P})\right)\right\} \\
& \underset{\sim}{\mathbf{w}_{\mathbf{g}}^{*} \mathbf{T}}(\mathbf{q}, \mathbf{P})=\left\{\frac{\partial}{\partial \mathbf{x}_{1}}\left(\frac{\partial^{2} \mathbf{w}^{*}}{\partial \mathbf{x}_{\mathbf{k}} \partial \mathbf{x}_{\mathbf{k}}}(\mathbf{q}, \mathbf{P})\right) \frac{\partial}{\partial \mathbf{x}_{2}}\left(\frac{\partial^{2} \mathbf{w}^{*}}{\partial \mathbf{x}_{\mathbf{k}} \partial \mathbf{x}_{\mathbf{k}}}(\mathbf{q}, \mathbf{P})\right)\right\}
\end{aligned}
$$

onde as derivadas dos deslocamentos e esforços fundamentais estão definidos no item (3.2.1).

Após a discretização do contorno e a aproximação das variáveis, os valores de $\mathbf{w} \mathbf{, ~}_{\mathbf{k k j}}$ em $\mathbf{N}_{\mathbf{i}}$ pontos internos são calculadas através da equação: 


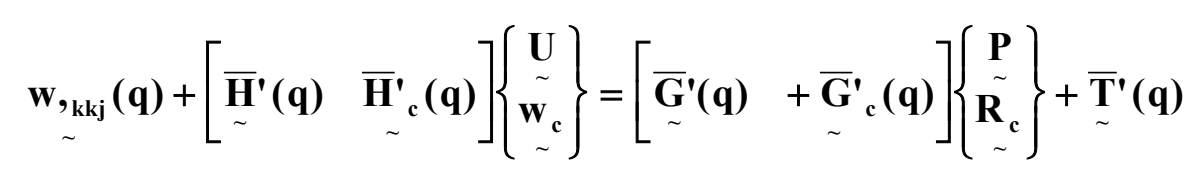

onde:

- os vetores $\{\mathbf{U}\},\left\{\mathbf{w}_{\mathbf{c}}\right\},\{\mathbf{P}\}$ e $\left\{\mathbf{R}_{\mathbf{c}}\right\}$ são os vetores de deslocamentos e esforços dos nós e cantos do contorno, já calculados,

- as matrizes $\underset{\sim}{\overline{\mathbf{H}^{\prime}}}$ e $\overline{\mathbf{G}}^{\prime}$ têm dimensões $2 \mathrm{~N}_{\mathrm{i}} \times 2 \mathrm{~N}_{\mathrm{n}}$ e $\overline{\mathbf{H}}_{c}^{\prime}$ e $\overline{\mathbf{G}}_{{ }_{\mathrm{c}}}^{\prime}$ têm dimensões $2 \mathrm{~N}_{\mathrm{i}} \times \mathrm{N}_{\mathrm{c}}$.

Os coeficientes de $\underset{\sim}{\overline{\mathbf{H}}}$ e $\bar{\sim}^{\overline{\mathbf{G}}^{\prime}}$ são provenientes das integrais sobre os elementos do contorno discretizado expressas em (4.19) e (4.20), porém com $\underset{\sim}{\mathbf{p}}(\mathbf{q}, \mathbf{P}) \underset{\sim}{\text { e } \underset{\sim}{\mathbf{u}}}(\mathbf{q}, \mathbf{P})$ definidos em (4.62) e (4.65).

As integrais de contorno da equação dos deslocamentos (4.47), da equação das curvaturas (4.57) e da equação das derivadas das curvaturas (4.69) podem ser feitas numericamente, já que não haverá singularidade nas funções fundamentais envolvidas.

\subsection{Momentos $\left(\mathrm{M}_{\mathrm{ij}}\right)$ nos Pontos do Contorno}

Os momentos em um ponto do contorno $\mathbf{Q}$ serão calculados em função dos valores nodais de deslocamentos e esforços do elemento ao qual pertence. Assim, seja o sistema local de coordenadas $\left(\overline{\mathbf{x}}_{1}, \overline{\mathbf{x}}_{2}\right)$ de um elemento qualquer do contorno, dado pela figura (4.11).

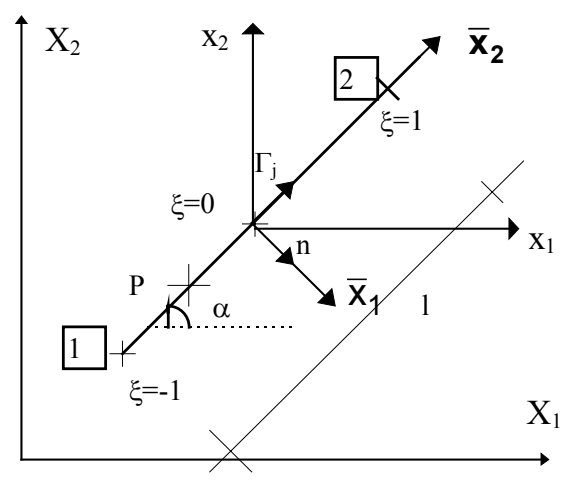

FIGURA 4.11 - Sistema Local de Coordenadas de um Elemento do Contorno 
Os valores nodais de $\mathbf{M}_{\mathbf{n}}=\overline{\mathbf{M}}_{\mathbf{1 1}}$ já foram obtidos através da solução do sistema de equações (4.36). Portanto, deve-se determinar as componentes dos momentos $\overline{\mathbf{M}}_{12}$ e $\overline{\mathbf{M}}_{22}$ em relação ao sistema local, e então, através da rotação do sistema de coordenadas, obteremse estes valores em relação ao sistema global $\left(\mathrm{X}_{1}, \mathrm{X}_{2}\right)$.

A partir da equação (2.9), obtém-se o valor de $\overline{\mathbf{M}}_{12}$ em função da curvatura w, $\mathbf{, 1 2}_{2}$ :

$$
\overline{\mathbf{M}}_{12}=-\mathbf{D}(1-v) \frac{\partial^{2} \mathbf{w}}{\partial \overline{\mathbf{x}}_{1} \partial \overline{\mathbf{x}}_{2}}
$$

Porém, de acordo com a equação (4.8), a rotação na direção normal $\partial \mathbf{w} / \partial \mathbf{n}$ é aproximada por uma função quadrática sobre o elemento $\Gamma_{\mathbf{j}}$. Como a direção $\overline{\mathbf{x}}_{\mathbf{1}}$ corresponde à direção normal n (ver figura 4.11), tem-se:

$$
\frac{\partial \mathbf{w}}{\partial \overline{\mathbf{x}}_{1}}=\frac{\partial \mathbf{w}}{\partial \mathbf{n}}=\left\{\phi_{1}(\mathbf{P}) \quad \phi_{2}(\mathbf{P}) \quad \phi_{3}(\mathbf{P})\right\}\left\{\begin{array}{c}
\frac{\partial \mathbf{w}^{1}}{\partial \mathbf{n}} \\
\frac{\partial \mathbf{w}^{2}}{\partial \mathbf{n}} \\
\frac{\partial \mathbf{w}^{3}}{\partial \mathbf{n}}
\end{array}\right\}
$$

Assim, derivando-se a equação (4.71) em relação à $\overline{\mathbf{x}}_{2}$, tem-se que:

$$
\frac{\partial^{2} \mathbf{w}}{\partial \overline{\mathbf{x}}_{1} \partial \overline{\mathbf{x}}_{2}}=\left\{\begin{array}{llll}
\frac{\partial \phi_{1}}{\partial \xi} \frac{\partial \xi}{\partial \overline{\mathbf{x}}_{2}} & \frac{\partial \phi_{2}}{\partial \xi} \frac{\partial \xi}{\partial \overline{\mathbf{x}}_{2}} & \frac{\partial \phi_{3}}{\partial \xi} \frac{\partial \xi}{\partial \overline{\mathbf{x}}_{2}}
\end{array}\right\}\left\{\begin{array}{c}
\frac{\partial \mathbf{w}^{1}}{\partial \mathbf{n}} \\
\frac{\partial \mathbf{w}^{2}}{\partial \mathbf{n}} \\
\frac{\partial \mathbf{w}^{3}}{\partial \mathbf{n}}
\end{array}\right\}
$$

Considerando-se a transformação de coordenadas dada por (4.1.b), tem-se que: 


$$
\frac{\partial^{2} \mathbf{w}}{\partial \overline{\mathbf{x}}_{1} \partial \overline{\mathbf{x}}_{2}}=\frac{\mathbf{2}}{\mathbf{l}}\left\{\begin{array}{lll}
\frac{\partial \phi_{1}(\mathbf{P})}{\partial \xi} & \frac{\partial \phi_{2}(\mathbf{P})}{\partial \xi} & \frac{\partial \phi_{3}(\mathbf{P})}{\partial \xi}
\end{array}\right\}\left\{\begin{array}{c}
\frac{\partial \mathbf{w}^{1}}{\partial \mathbf{n}} \\
\frac{\partial \mathbf{w}^{2}}{\partial \mathbf{n}} \\
\frac{\partial \mathbf{w}^{3}}{\partial \mathbf{n}}
\end{array}\right\}
$$

Calculando-se as derivadas das funções aproximadoras, dadas em (4.11) e substituindo-se em (4.70), chega-se à equação :

$$
\overline{\mathbf{M}}_{12}=-\frac{2 \mathbf{D}(1-v)}{\mathbf{l}}\left[\frac{\left(\xi_{3}-2 \xi\right)}{\xi_{1}\left(\xi_{3}-\xi_{1}\right)} \frac{\partial \mathbf{w}^{1}}{\partial \mathbf{n}}+\frac{\left(2 \xi-\xi_{1}-\xi_{3}\right)}{\xi_{1} \xi_{3}} \frac{\partial \mathbf{w}^{2}}{\partial \mathbf{n}}+\frac{\left(\xi_{1}-2 \xi\right)}{\xi_{3}\left(\xi_{1}-\xi_{3}\right)} \frac{\partial \mathbf{w}^{3}}{\partial \mathbf{n}}\right]
$$

onde:

- $\xi_{1}$ e $\xi_{3}$ são as coordenadas homogêneas dos nós 1 e 3, respectivamente (ver figura 4.6). Nesse trabalho, adota-se $\xi_{1}=-1$ e $\xi_{3}=1$.

- é a coordenada adimensional do ponto $\mathbf{P}$.

Considerando-se a equação (2.9), obtêm-se as expressões para $\overline{\mathbf{M}}_{22}$ e $\overline{\mathbf{M}}_{11}$ :

$$
\begin{aligned}
& \overline{\mathbf{M}}_{22}=-\mathbf{D}\left(v \frac{\partial^{2} \mathbf{w}}{\partial \overline{\mathbf{x}}_{1}^{2}}+\frac{\partial^{2} \mathbf{w}}{\partial \overline{\mathbf{x}}_{2}^{2}}\right) \\
& \overline{\mathbf{M}}_{11}=\mathbf{M}_{\mathrm{n}}=-\mathbf{D}\left(\frac{\partial^{2} \mathbf{w}}{\partial \overline{\mathbf{x}}_{1}^{2}}+v \frac{\partial^{2} \mathbf{w}}{\partial \overline{\mathbf{x}}_{2}^{2}}\right)
\end{aligned}
$$

A partir da equação (4.76), obtém-se:

$$
\frac{\partial^{2} \mathbf{w}}{\partial \overline{\mathbf{x}}_{1}^{2}}=-\frac{\mathbf{M}_{\mathbf{n}}}{\mathbf{D}}-v \frac{\partial^{2} \mathbf{w}}{\partial \overline{\mathbf{x}}_{2}^{2}}
$$

Substituindo-se (4.77) em (4.75), obtém-se:

$$
\overline{\mathbf{M}}_{22}=-\mathbf{D}\left(1-v^{2}\right) \frac{\partial^{2} \mathbf{w}}{\partial \overline{\mathbf{x}}_{2}^{2}}+v \mathbf{M}_{\mathbf{n}}
$$


De acordo com (4.8), w é aproximada por uma função quadrática sobre o elemento $\Gamma_{\mathbf{j}}$. Assim, derivando-se duas vezes a expressão de $\mathbf{w}$, em relação à $\overline{\mathbf{x}}_{\mathbf{2}}$ e fazendo-se a transformação de coordenadas, dada por (4.1.b), pode-se escrever:

$$
\frac{\partial^{2} \mathbf{w}}{\partial \overline{\mathbf{x}}_{2}^{2}}=\frac{4}{\mathbf{l}^{2}}\left(\frac{\partial^{2} \phi_{1}(\mathbf{P})}{\partial \xi^{2}} \mathbf{w}^{1}+\frac{\partial^{2} \phi_{2}(\mathbf{P})}{\partial \xi^{2}} \mathbf{w}^{2}+\frac{\partial^{2} \phi_{3}(\mathbf{P})}{\partial \xi^{2}} \mathbf{w}^{3}\right)
$$

Calculando-se as derivadas das funções aproximadoras, obtém-se a expressão de W,22. Substituindo-se essa última em (4.78), chega-se à equação:

$$
\overline{\mathbf{M}}_{22}=\frac{\mathbf{D}\left(1-v^{2}\right)}{\mathbf{l}^{2}}\left[\frac{8}{\xi_{1}\left(\xi_{3}-\xi_{1}\right)} \mathbf{w}^{1}-\frac{8}{\xi_{1} \xi_{3}} \mathbf{w}^{2}+\frac{8}{\xi_{3}\left(\xi_{1}-\xi_{3}\right)} \mathbf{w}^{3}\right]+v \mathbf{M}_{\mathbf{n}}
$$

A transformação de coordenadas do sistema local $\left(\overline{\mathbf{x}}_{1}, \overline{\mathbf{x}}_{2}\right)($ ver figura 4.11$)$ para o sistema local $\left(\mathrm{x}_{1}, \mathrm{x}_{2}\right)$, associado aos eixos de referência do sistema global $\left(\mathrm{X}_{1}, \mathrm{X}_{2}\right)$, e, viceversa, são dadas por:

$$
\begin{aligned}
& \left\{\begin{array}{l}
\mathbf{x}_{1} \\
\mathbf{x}_{2}
\end{array}\right\}=\left[\begin{array}{cc}
\operatorname{sen} \alpha & \cos \alpha \\
-\cos \alpha & \operatorname{sen} \alpha
\end{array}\right]\left\{\begin{array}{l}
\overline{\mathbf{x}}_{1} \\
\bar{x}_{2}
\end{array}\right\} \\
& \left\{\begin{array}{l}
\bar{x}_{1} \\
\bar{x}_{2}
\end{array}\right\}=\left[\begin{array}{cc}
\operatorname{sen} \alpha & -\cos \alpha \\
\cos \alpha & \operatorname{sen} \alpha
\end{array}\right]\left\{\begin{array}{l}
\mathbf{x}_{1} \\
x_{2}
\end{array}\right\}
\end{aligned}
$$

Considerando-se as equações (4.81) e (4.82), obtém-se a transformação do vetor de momentos no sistema local $\left(\overline{\mathbf{x}}_{1}, \overline{\mathbf{x}}_{2}\right)$ para o sistema global $\left(\mathrm{X}_{1}, \mathrm{X}_{2}\right)$, que é dada por:

$$
\left\{\begin{array}{l}
M_{11} \\
M_{12} \\
M_{22}
\end{array}\right\}=\left[\begin{array}{ccc}
\operatorname{sen}^{2} \alpha & 2 \operatorname{sen} \alpha \cos \alpha & \cos ^{2} \alpha \\
-\operatorname{sen} \alpha \cos \alpha & -\cos ^{2} \alpha+\operatorname{sen}^{2} \alpha & \operatorname{sen} \alpha \cos \alpha \\
\cos ^{2} \alpha & -2 \operatorname{sen} \alpha \cos \alpha & \operatorname{sen}^{2} \alpha
\end{array}\right]\left\{\begin{array}{l}
\bar{M}_{11} \\
\bar{M}_{12} \\
\bar{M}_{22}
\end{array}\right\}
$$

Fazendo-se $\mathbf{M}_{\mathbf{n}}=\overline{\mathbf{M}}_{11}$ e substituindo-se os valores de $\overline{\mathbf{M}}_{12}$ e $\overline{\mathbf{M}}_{22}$, dados por (4.74) e (4.80), respectivamente, na equação (4.83) obtêm-se os momentos no ponto $\mathbf{Q}$ em função dos deslocamentos e esforços nodais do elemento $\mathbf{j}$ ao qual pertence, ou seja: 


$$
\left\{\begin{array}{l}
\mathbf{M}_{11} \\
\mathbf{M}_{12} \\
\mathbf{M}_{22}
\end{array}\right\}_{\mathbf{Q}}=[\mathbf{h}]_{\mathrm{j}}\{\mathbf{U}\}_{\mathrm{j}}+[\mathbf{g}]_{\mathrm{j}}\{\mathbf{P}\}_{\mathrm{j}}
$$

onde as matrizes $\mathbf{h}$ e $\mathbf{g}$ têm dimensões (3x6) e $\mathbf{U}$ e $\mathbf{P}$ são vetores de dimensão (6x1), que trazem os valores nodais dos deslocamentos e esforços do elemento $\mathbf{j}$ em questão.

Após acoplar todos os elementos que compõe o contorno, chega-se à equação matricial de momentos para $\mathbf{N}_{\mathbf{n}}$ pontos do contorno, que é dada por:

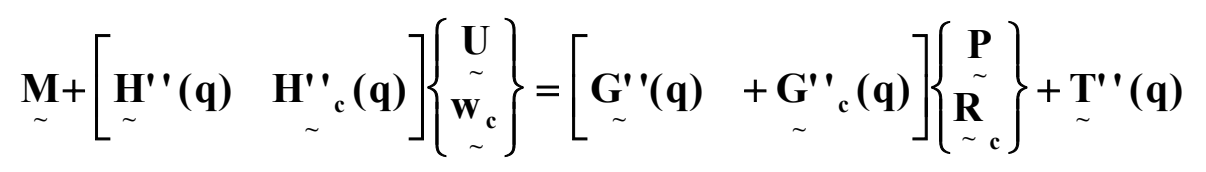

onde:

- os vetores $\{\mathbf{U}\},\left\{\mathbf{w}_{\mathbf{c}}\right\},\{\mathbf{P}\}$ e $\left\{\mathbf{R}_{\mathbf{c}}\right\}$ são os vetores de deslocamentos e esforços dos nós e cantos do contorno, já calculados,

- as matrizes [H"] e [G”] têm dimensões $3 \mathrm{~N}_{\mathrm{n}} \times 2 \mathrm{~N}_{\mathrm{n}}$,

- [H”c], [G”], têm dimensões $3 \mathrm{~N}_{\mathrm{n}} \times \mathrm{N}_{\mathrm{c}}$, mas são matrizes nulas.

$\mathrm{Na}$ montagem destas matrizes para os nós comuns a dois elementos distintos, que não sejam nós duplos, adota-se a média entre os coeficientes relativos a cada um dos elementos.

\subsection{Integração Numérica Sobre os Elementos}

\subsubsection{Integração Normal}

Para os casos em que o ponto de carregamento ou ponto de colocação não pertence ao elemento a ser integrado, as integrais sobre os elementos podem ser calculadas numericamente. Como no caso de uma equação relativa a um ponto interno, quer seja a de deslocamento (4.47), a de curvaturas (4.57) ou a da derivada das curvaturas (4.69), o ponto de carregamento interno q não pertence a nenhum elemento do contorno, as integrais sobre os elementos que aparecem nas mesmas poderão sempre ser feitas numericamente. O mesmo ocorre com a equação de deslocamento escrita para um ponto de carregamento externo $\mathbf{A}$. Contudo, quando o ponto de carregamento é um ponto do contorno $\mathbf{Q}$, as integrais somente poderão ser feitas numericamente, se o mesmo não pertencer ao elemento considerado. 
Adotando-se esquemas particulares de integração, as integrais poderiam ser calculadas numericamente, para o caso em que $\mathbf{Q}$ pertence ao elemento que está sendo integrado, porém, isso não será feito nesse trabalho.

A fim de fazer a integração numérica, é conveniente que essas expressões sejam escritas em função de coordenadas homogêneas. Assim, tomando-se como exemplo as integrais sobre os elementos, que aparecem na equação de deslocamento para um ponto do contorno $\mathbf{Q}$, dadas por (4.19) e (4.20), e fazendo-se a mudança de coordenadas nas mesmas, dada por (4.1.b), têm-se:

$$
\begin{aligned}
& \mathbf{h}_{\mathbf{k}}^{\mathrm{n}}(\mathbf{Q})=\frac{\mathbf{l}}{2} \int_{-1}^{1} \mathbf{p}_{\mathrm{k}}^{*}(\mathbf{Q}, \mathbf{P}) \phi_{\mathrm{n}}(\mathbf{P}) \mathbf{d} \xi(\mathbf{P}) \quad(\mathrm{n}=1,2,3) \quad(\mathrm{k}=1,2) \\
& \mathbf{g}_{\mathbf{k}}^{\mathrm{n}}(\mathbf{Q})=\frac{\mathbf{l}}{2} \int_{-1}^{1} \mathbf{u}_{\mathrm{k}}^{*}(\mathbf{Q}, \mathbf{P}) \phi_{\mathrm{n}}(\mathbf{P}) \mathbf{d} \xi(\mathbf{P})
\end{aligned}
$$

onde:

- $\mathbf{n}$ é o nó local do elemento onde se mede a resposta da carga unitária,

- $\mathbf{k}$ indica a natureza da resposta medida em $\mathbf{P}$. Assim, $\mathbf{p}_{1}^{*}=\mathbf{V}_{\mathbf{n}}^{*}, \mathbf{p}_{2}^{*}=\mathbf{M}_{\mathrm{nn}}^{*}, \mathbf{u}_{1}^{*}=\mathbf{w}^{*} \mathrm{e}$ $\mathbf{u}_{2}^{*}=\partial \mathbf{w}^{*} / \partial \mathbf{n}$, dados por (3.33), (3.30), (3.25) e (3.27), respectivamente.

De cada integral (4.86) e (4.87), resultarão seis coeficientes, pois em cada elemento tem-se três nós e duas variáveis por nó. A integração numérica é feita pela fórmula de quadratura de Gauss, que é dada pela seguinte expressão:

$$
\int_{-1}^{1} \mathbf{f}(\xi) \mathbf{d} \xi=\sum_{i=1}^{N_{g}} \mathbf{f}\left(\xi_{i}\right) W_{i}
$$

onde:

- $\mathbf{f}(\xi)$ é a função a ser integrada, escrita em relação à coordenada $\xi$,

- $\mathbf{N}_{\mathrm{g}}$ é o número de pontos de integração,

- $\xi_{\mathrm{i}}$ é a coordenada adimensional do ponto $\mathbf{i}$ de integração, definida em função do $\mathbf{N}_{\mathrm{g}}$,

- $\mathbf{W}_{\mathbf{i}}$ é o fator ponderador, também definido em função de $\mathbf{N}_{\mathbf{g}}$.

No caso da integração numérica não ser feita através de sub-elementos, o número $\mathbf{N}_{\mathrm{g}}$ de pontos de integração deve ser escolhido em função da distância entre o ponto $\mathbf{Q}$ de carregamento e o elemento a ser integrado, o comprimento do elemento e a função a ser 
integrada ( GIL RODRIGUEZ, 1986 ), considerando-se, ainda, a precisão que se pretende alcançar.

Os termos do vetor de cargas $\mathbf{T}$, que são obtidos a partir de (3.72), através da integração sobre os segmentos lineares em que o contorno $\Gamma_{\mathrm{g}}$ do carregamento é dividido, também podem ser feitas numericamente, através da fórmula de quadratura de Gauss, aplicando-se a mudança de coordenadas, dada por (4.1.b), na expressão (3.72). Nesse caso, os valores obtidos com a integração numérica podem ser exatos, já que não se faz nenhum tipo de aproximação nas integrais, considerando que o carregamento seja linear. No caso do contorno $\Gamma_{\mathrm{g}}$ do carregamento coincidir com o contorno da placa, em uma determinada região, pode ocorrer de se ter o ponto de carregamento $\mathbf{Q}$ pertencente ao segmento a ser integrado. Neste caso, porém, as integrais são nulas, uma vez que o produto escalar $\mathbf{r}_{\mathbf{i}} \cdot \mathbf{n}_{\mathbf{i}}$ da equação (3.72) é nulo, para qualquer ponto do segmento.

\subsubsection{Técnica de Sub-elementos}

Quanto mais perto estiver o ponto de colocação $\mathbf{A}, \mathbf{Q}$ ou $\mathbf{q}$ do elemento a ser integrado, maior será a influência desse no valor da variável do ponto considerado. Para se ter uma boa precisão no cálculo, a distância entre o ponto de colocação e o ponto médio do elemento não pode ser muito grande e quanto menor essa distância, maior deve ser o número de pontos de Gauss usados na integração. Assim, a técnica de sub-elementos, adotada nesse trabalho, consiste em dividir o elemento, considerado na integração, em sub-elementos, de modo que a distância entre o ponto de colocação e o ponto médio do mesmo não seja menor que o seu comprimento, como é mostrado na figura (4.12).

Se $\varphi \leq \mathbf{6 0}$, o comprimento do sub-elemento será tal que forme um triângulo isósceles entre o seu nó inicial, seu nó final e A (Figura 4.12), e portanto será dado por:

$$
\mathbf{d}=\frac{\mathbf{r s}}{2 \cos \varphi}
$$

Se $\varphi>60$, o comprimento do sub-elemento será igual à distância entre $\mathbf{A}$ e o nó final do sub-elemento anterior (Figura 4.12). 


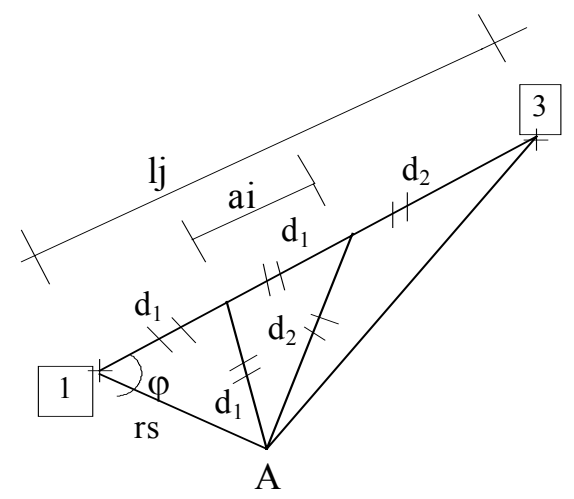

FIGURA 4.12 - Divisão de um Elemento em Sub-elementos

onde: rs é a distância ao nó inicial do sub-elemento, ai é o comprimento do sub-elemento $\mathbf{i}$, lj é o comprimento do elemento $\mathbf{j}$, $\varphi$ é o ângulo entre rs e o elemento $\mathbf{j}$.

O sub-elemento terá um sistema de coordenadas homogêneas equivalente ao utilizado para o elemento (Figura 4.2), onde a coordenada adimensional será dada por $\eta$. $\mathrm{O}$ número de pontos de Gauss usado na integração de cada sub-elemento será definido em função da distância rs (ver figura 4.12) e do comprimento do sub-elemento $\mathbf{a}_{\mathbf{i}}$ do seguinte modo:

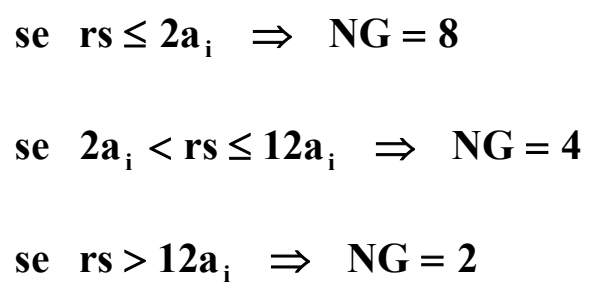

As coordenadas cartesianas dos pontos de Gauss são calculadas a partir das coordenadas cartesianas dos nós do sub-elemento e de $\eta$, como indica a equação (4.3). Então, a partir das coordenadas cartesianas, determina-se a coordenada $\xi_{\text {IG }}$ do ponto de Gauss em consideração, através de uma das equações (4.2), sendo $\xi$ a coordenada adimensional relativa ao sistema local do elemento. Com $\xi_{\text {IG }}$ determina-se o valor das funções de forma $\phi_{1}, \phi_{2}$ e $\phi_{3}$, dadas pelas equações (4.10), e finalmente faz-se o cálculo do integrando. Logo, as integrais (4.86) e (4.87) serão calculadas da seguinte maneira: 


$$
\int_{-1 / 2}^{1 / 2} \mathbf{F} \phi \mathbf{d} \Gamma_{j}=\sum_{i=1}^{N_{\text {sube }}} \int_{-\mathbf{a} / 2}^{\mathbf{a} / 2} \mathbf{F} \phi \mathbf{d} \Gamma_{\mathbf{i}}=\sum_{\mathrm{i}=1}^{\mathrm{N}_{\text {sube }}} \frac{\mathbf{a}}{2} \int_{-1}^{1} \mathbf{F} \phi \mathbf{d} \eta=\sum_{\mathrm{i}=1}^{\mathrm{N}_{\text {sube }}} \frac{\mathbf{a}}{2} \sum_{\mathrm{IG}=\mathbf{1}}^{\mathbf{N}_{\mathrm{g}}}(\mathbf{F}(\xi) \phi(\xi))_{\mathrm{IG}} \mathbf{W}_{\mathrm{IG}}
$$

A técnica de sub-elementos é empregada nas integrais sobre os elementos e sobre o contorno do carregamento, que aparecem na equação de deslocamento de um ponto interno, de um ponto externo ou de um ponto do contorno, e também nas equações das curvaturas e derivadas das curvaturas de pontos internos.

\subsection{Integração Analítica sobre os Elementos}

Quando o ponto de colocação $\mathbf{Q}$ pertence ao elemento a ser integrado, as funções fundamentais envolvidas nas integrações apresentam singularidades. Nesses casos, as integrais (4.86) e (4.87) devem ser feitas analiticamente, sendo que no caso de singularidades do tipo $\mathbf{1} / \mathbf{r}$, as integrais são interpretadas no sentido do valor principal de Cauchy. Assim, considerando-se um nó $\mathbf{n}$ local do elemento ao qual $\mathbf{Q}$ pertence, as integrais (4.86) e (4.87) são expressas por:

$$
\begin{aligned}
& \mathbf{h}_{1}^{\mathrm{n}}(\mathbf{Q})=\frac{1}{2} \int_{-1}^{1} \mathbf{V}_{\mathrm{n}}^{*}(\mathbf{Q}, \mathbf{P}) \phi_{\mathrm{n}}(\mathbf{P}) \mathrm{d} \xi(\mathrm{P}) \quad(\mathrm{n}=1,2,3) \\
& \mathbf{h}_{2}^{\mathrm{n}}(\mathbf{Q})=-\frac{1}{2} \int_{-1}^{1} \mathbf{M}_{\mathrm{n}}^{*}(\mathbf{Q}, \mathbf{P}) \phi_{\mathrm{n}}(\mathbf{P}) \mathrm{d} \xi(\mathrm{P}) \\
& \mathbf{g}_{1}^{\mathrm{n}}(\mathbf{Q})=\frac{1}{2} \int_{-1}^{1} \mathbf{w}^{*}(\mathbf{Q}, \mathbf{P}) \phi_{\mathrm{n}}(\mathbf{P}) \mathrm{d} \xi(\mathrm{P}) \\
& \mathbf{g}_{2}^{\mathrm{n}}(\mathbf{Q})=-\frac{1}{2} \int_{-1}^{1} \frac{\partial \mathbf{w}^{*}}{\partial \mathbf{n}}(\mathbf{Q}, \mathbf{P}) \phi_{\mathrm{n}}(\mathbf{P}) \mathrm{d} \xi(\mathbf{P})
\end{aligned}
$$

Porém, no caso em que $\mathbf{Q}$ pertence ao elemento a ser integrado, tem-se que $\mathbf{r}_{\mathbf{i}} \cdot \mathbf{n}_{\mathrm{i}}=\cos \left(90^{\circ}\right)=\mathbf{0}$, e portanto, conclui-se que as expressões de $\mathbf{V}_{\mathbf{n}}^{*}$ e $\partial \mathbf{w}^{*} / \partial \mathbf{n}$, dadas respectivamente por (3.33) e (3.27), são nulas. As coordenadas do ponto singular $\mathbf{Q}$ são obtidas a partir das coordenadas dos nós extremos (1 e 3), utilizando-se as funções de forma $\phi_{\mathrm{g} 1}$ e $\phi_{\mathrm{g} 2}$, como mostra a equação (4.3). Assim, substituindo-se as expressões dos deslocamentos e esforços fundamentais dadas no item (3.2.1) e as funções aproximadoras 
das variáveis ,dadas por (4.11), nas equações (4.91) a (4.94), fazendo-se a integração e considerando-se as funções $\phi_{\mathrm{g} 1}$ e $\phi_{\mathrm{g} 2}$, dadas pelas equações (4.4), obtêm-se:

$$
\begin{aligned}
& \mathbf{h}_{1}^{\mathrm{n}}(\mathbf{Q})=\mathbf{0} \\
& \mathbf{h}_{2}^{\mathrm{n}}(\mathbf{Q})=\frac{\ell}{4 \pi}\left\{\mathbf{C}_{1}^{\mathrm{n}}(1+v)\left[\phi_{\mathrm{g} 2 \mathrm{~s}} \cdot \ln \left(\ell \cdot \phi_{\mathrm{g} 2 \mathrm{~s}}\right)+\phi_{\mathrm{g} 1 \mathrm{~s}} \cdot \ln \left(\ell \cdot \phi_{\mathrm{g} 1 \mathrm{~s}}\right)-1\right]+\right. \\
& +\mathbf{C}_{2}^{\mathrm{n}}(1+v)\left[\left(\phi_{\mathrm{g} 2 \mathrm{~s}}\right)^{2} \ln \left(\ell \cdot \phi_{\mathrm{g} 2 \mathrm{~s}}\right)-\left(\phi_{\mathrm{g} 1 \mathrm{~s}}\right)^{2} \ln \left(\ell \cdot \phi_{\mathrm{g} 1 \mathrm{~s}}\right)-\frac{\xi_{\mathrm{s}}}{2}\right]+ \\
& +\mathbf{C}_{3}^{\mathrm{n}}(1+v)\left[\frac{4}{3}\left(\phi_{\mathrm{g} 2 \mathrm{~s}}\right)^{3} \ln \left(\ell \cdot \phi_{\mathrm{g} 2 \mathrm{~s}}\right)+\frac{4}{3}\left(\phi_{\mathrm{g} 1 \mathrm{~s}}\right)^{3} \ln \left(\ell \cdot \phi_{\mathrm{g} 1 \mathrm{~s}}\right)-\frac{1}{9}\left(1+3 \xi_{\mathrm{s}}^{2}\right)\right]+ \\
& \left.\quad+v\left[\mathbf{C}_{1}^{\mathrm{n}}+\mathbf{C}_{2}^{\mathrm{n}} \xi_{\mathrm{s}}+\frac{\mathbf{C}_{3}^{\mathrm{n}}}{3}\left(1+3 \xi_{\mathrm{s}}^{2}\right)\right]\right\} \\
& \mathbf{g}_{1}^{\mathrm{n}}(\mathbf{Q})=\frac{\ell^{3}}{8 \pi \mathrm{D}}\left\{\frac{\mathbf{C}_{1}^{\mathrm{n}}}{3}\left[\left(\phi_{\mathrm{g} 2 \mathrm{~s}}\right)^{2}\left(\ln \left(\ell \cdot \phi_{\mathrm{g} 2 \mathrm{~s}}\right)-\frac{5}{6}\right)^{\mathrm{n}}+\left(\phi_{\mathrm{g} 1 \mathrm{~s}}\right)^{3}\left(\ln \left(\ell \cdot \phi_{\mathrm{g} 1 \mathrm{~s}}\right)-\frac{5}{6}\right)\right]+\right. \\
& +\frac{\mathbf{C}_{2}^{\mathrm{n}}}{2}\left[\left(\phi_{\mathrm{g} 2 \mathrm{~s}}\right)^{4}\left(\ln \left(\ell \cdot \phi_{\mathrm{g} 2 \mathrm{~s}}\right)-\frac{3}{4}\right)-\left(\phi_{\mathrm{g} 1 \mathrm{~s}}\right)^{4}\left(\ln \left(\ell \cdot \phi_{\mathrm{g} 1 \mathrm{~s}}\right)-\frac{3}{4}\right)\right]+ \\
& \left.+\frac{4 . \mathbf{C}_{3}^{\mathrm{n}}}{\mathbf{5}}\left[\left(\phi_{\mathrm{g} 2}\right)^{5}\left(\ln \left(\ell \cdot \phi_{\mathrm{g} 2 \mathrm{~s}}\right)-\frac{7}{10}\right)+\left(\phi_{\mathrm{g} 1 \mathrm{~s}}\right)^{5}\left(\ln \left(\ell \cdot \phi_{\mathrm{g} 1 \mathrm{~s}}\right)-\frac{7}{10}\right)\right]\right\}
\end{aligned}
$$

onde $\xi_{\text {s }}$ é a coordenada adimensional do ponto $\mathbf{Q}$ (ver figura 4.12), $\mathbf{I}$ é o comprimento do elemento, e $\phi_{\mathrm{g} 1 \mathrm{~s}}$ e $\phi_{\mathrm{g} 2 \mathrm{~s}}$, são os valores das funções de forma $\phi_{\mathrm{g} 1}$ e $\phi_{\mathrm{g} 2}$, dadas por (4.4.a) e (4.4.b), no ponto $\mathbf{Q}$. 


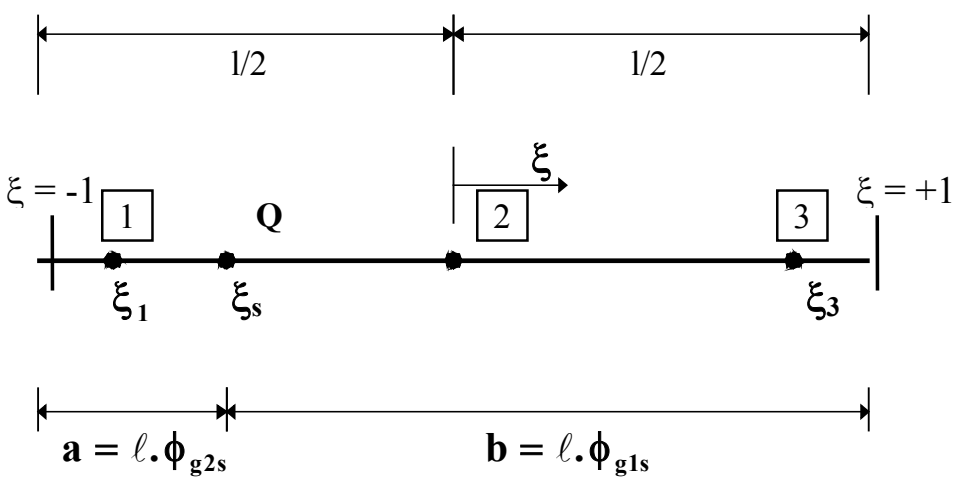

FIGURA 4.12 - Elemento de Contorno Descontínuo

Nestas expressões, as constantes $\mathbf{C}_{\mathbf{1}}^{\mathbf{n}}, \mathbf{C}_{\mathbf{2}}^{\mathbf{n}}, \mathbf{C}_{\mathbf{3}}^{\mathbf{n}}$ são relativas aos nós locais e portanto, dependem das funções aproximadoras $\phi_{1}, \phi_{2}, \phi_{3}$. Estes valores podem ser escritos, genericamente, na forma:

$$
\begin{aligned}
\mathbf{C}_{1}^{\mathbf{n}} & =\frac{-\xi_{\mathrm{s}}^{2}+\left(\xi_{\mathrm{i}}+\xi_{\mathrm{j}}\right) \cdot \xi_{\mathrm{s}}-\xi_{\mathrm{i}} \cdot \xi_{\mathrm{j}}}{\left(\xi_{\mathrm{i}}-\xi_{\mathrm{n}}\right) \cdot\left(\xi_{\mathrm{n}}-\xi_{\mathrm{j}}\right)} \\
\mathbf{C}_{2}^{\mathbf{n}} & =\frac{2 \cdot \xi_{\mathrm{s}}-\left(\xi_{\mathrm{i}}+\xi_{\mathrm{j}}\right)}{\left(\xi_{\mathrm{i}}-\xi_{\mathrm{n}}\right) \cdot\left(\xi_{\mathrm{n}}-\xi_{\mathrm{j}}\right)} \\
\mathbf{C}_{3}^{\mathbf{n}} & =\frac{-1}{\left(\xi_{i}-\xi_{n}\right) \cdot\left(\xi_{n}-\xi_{j}\right)}
\end{aligned}
$$

onde $\mathbf{n}, \mathbf{i}, \mathbf{j}=\mathbf{1}, \mathbf{2}, \mathbf{3}$ e $\mathbf{n} \neq \mathbf{i} \neq \mathbf{j}$.

Nesse trabalho, os nós 1 e 3 (figura 4.12) coincidem com as extremidades dos elementos e, portanto, têm-se $\xi_{1}=\mathbf{- 1}$ e $\xi_{3}=\mathbf{1}$. Assim, substituindo-se esses valores nas equações (4.99), obtêm-se as expressões de $\mathbf{C}_{\mathbf{1}}^{\mathbf{n}}, \mathbf{C}_{\mathbf{2}}^{\mathbf{n}}$ e $\mathbf{C}_{\mathbf{3}}^{\mathbf{n}}$ para esse caso particular.

\subsection{Exemplos Numéricos}

A seguir, serão apresentados alguns exemplos de aplicação da formulação elástica de placas desenvolvida até o presente capítulo. Serão utilizadas diversas condições de contorno e carregamento e os resultados obtidos serão comparados com as soluções 
analíticas dadas por TIMOSHENKO (1959) e com soluções numéricas que utilizam o Método dos Elementos de Contorno, como o trabalho de PAIVA (1987), CALDERÓN (1991), os quais utilizam elementos de função aproximadora linear, e CHUEIRI (1994), que utiliza aproximações quadráticas.

Em todos os exemplos apresentados a posição do nó duplo foi definida pela coordenada adimensional $\xi_{\mathrm{s}}=\mathbf{\pm 0 , 6}$, embora a variação no valor de $\xi_{\mathrm{s}}$ entre 0,4 e 0,6 não produza alterações significativas nas soluções e o coeficiente de Poisson $v$ utilizado foi igual a 0,3 para os exemplos de placa.

4.9.1 Placa quadrada com dois lados opostos apoiados e os demais engastados, submetida a carregamento uniforme

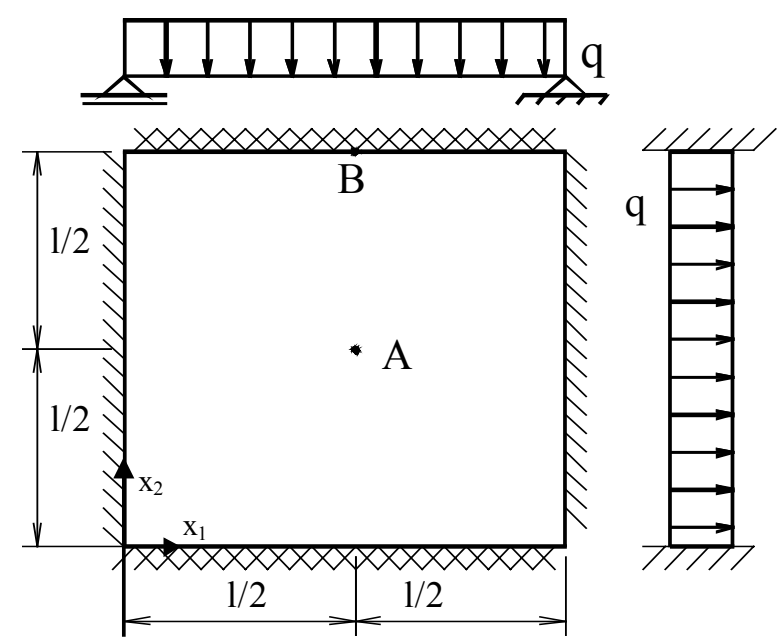

FIGURA 4.13 - Placa Quadrada Apoiada em Dois Lados Opostos e Engastada nos outros Dois.

A placa apresentada na figura (4.13) foi calculada utilizando-se pontos de colocação no contorno $\mathbf{Q}$ e externos A (ver figura 4.7.a). O contorno foi discretizado em 16 elementos, o que resulta em 36 pontos nodais no contorno, como está mostrado na figura (4.14), onde os nós 1 e 36, 9 e 10, 18 e 19, 27 e 28 são nós duplos, definidos com as mesmas coordenadas. Um dos objetivos do exemplo é analisar a influência da posição dos pontos de colocação externos nos deslocamentos e momentos fletores dos pontos A e B (ver figura 4.13), através da variação do valor de a, que está indicado na equação (4.27). 


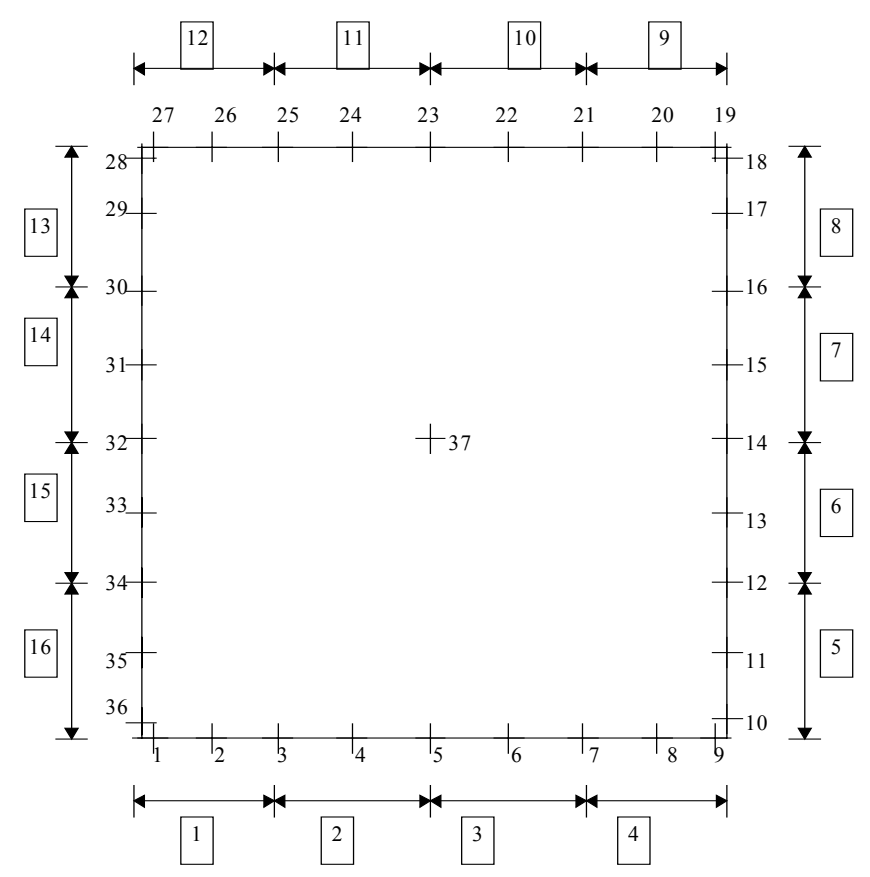

FIGURA 4.14 - Discretização da Placa

$\mathrm{Na}$ tabela (4.1), estão indicados os resultados obtidos, a solução analítica de TIMOSHENKO (1959) e as soluções numéricas obtidas por CALDERON (1991) e CHUEIRI (1994).

Pode-se observar nos resultados apresentados por CHUEIRI (1994), os quais foram obtidos considerando-se $\mathrm{a}=0,25$, que a formulação proposta apresenta excelentes resultados, mesmo quando utilizam-se apenas 4 elementos para a discretização do contorno. Analisandose, ainda, a tabela (4.1), conclui-se que a posição dos pontos externos tem maior influência sobre os valores dos momentos do ponto $\mathbf{B}$, sendo que os resultados que mais se aproximam da resposta analítica dada por TIMOSHENKO (1959), foram os obtidos para a $=0,0001$. Comparando-se ainda os resultados obtidos para $\mathrm{a}=0,25$, com aqueles apresentados por CHUEIRI (1994), conclui-se, que nesse caso, a técnica de sub-elementos não tem influência significativa na solução.

TABELA 4.1 - Resultados do exemplo 1

\begin{tabular}{cccccc}
\hline SOLUÇÕES & \multicolumn{3}{c}{ PONTO A } & \multicolumn{2}{c}{ PONTO B } \\
\hline & $\mathbf{w} /\left(\mathbf{q} \mathbf{l}^{\mathbf{4}} / \mathbf{D}\right)$ & $\mathbf{M}_{\mathbf{1 1}} / \mathbf{q} \mathbf{l}^{\mathbf{2}}$ & $\mathbf{M}_{\mathbf{2 2}} / \mathbf{q l}^{\mathbf{2}}$ & $\mathbf{M}_{\mathbf{1 1}} / \mathbf{q} \mathbf{l}^{\mathbf{2}}$ & $\mathbf{M}_{\mathbf{2 2}} / \mathbf{q l}^{\mathbf{2}}$ \\
\hline TIMOSHENKO & 0,00192 & 0,0244 & 0,0332 & $-0,0209$ & $-0,0697$ \\
\hline CALDERON & 0,00192 & 0,0244 & 0,0332 & $-0,0203$ & $-0,0701$ \\
\hline
\end{tabular}




\begin{tabular}{cccccc}
\hline (40 elem. $)$ & & & & & \\
\hline CHUEIRI & & & & & \\
$(4$ elem. $)$ & 0,00192 & 0,0244 & 0,0332 & $-0,0207$ & $-0,0689$ \\
$(8$ elem. $)$ & 0,00192 & 0,0244 & 0,0332 & $-0,0214$ & $-0,0713$ \\
$(16$ elem. $)$ & 0,00192 & 0,0244 & 0,0332 & $-0,0209$ & $-0,0699$ \\
\hline PRESENTE & & & & & \\
ESTUDO & & & & & \\
$(\mathrm{a}=0,0001)$ & 0,00192 & 0,0244 & 0,0333 & $-0,0209$ & $-0,0697$ \\
$(\mathrm{a}=0,001)$ & 0,00192 & 0,0244 & 0,0333 & $-0,0209$ & $-0,0698$ \\
$(\mathrm{a}=0,01)$ & 0,00192 & 0,0244 & 0,0333 & $-0,0210$ & $-0,0699$ \\
$(\mathrm{a}=0,1)$ & 0,00192 & 0,0244 & 0,0333 & $-0,0210$ & $-0,0699$ \\
$(\mathrm{a}=0,25)$ & 0,00192 & 0,0244 & 0,0332 & $-0,0210$ & $-0,06999$ \\
$(\mathrm{a}=0,5)$ & 0,00192 & 0,0244 & 0,0332 & $-0,0206$ & $-0,0703$ \\
$(\mathrm{a}=0,7)$ & 0,00192 & 0,0244 & 0,0332 & $-0,0212$ & $-0,0705$ \\
$(\mathrm{a}=1,0)$ & 0,00192 & 0,0244 & 0,0332 & $-0,0213$ & $-0,0710$ \\
$(\mathrm{a}=1,5)$ & 0,00192 & 0,0244 & 0,0332 & $-0,0215$ & $-0,0717$ \\
$(\mathrm{a}=2,0)$ & 0,00192 & 0,0244 & 0,0332 & $-0,0217$ & $-0,0723$ \\
\hline
\end{tabular}

\subsubsection{Placa quadrada apoiada nos quatro cantos e submetida a carga uniformemente distribuída}

A placa indicada na figura (4.15), que apresenta os lados livres e apoios somente nos cantos, será analisada discretizando-se o contorno em 4 e 16 elementos (ver figura 4.14), considerando-se pontos de colocação no contorno e externos, sendo que, para esses últimos, utilizou-se diferentes posições. 


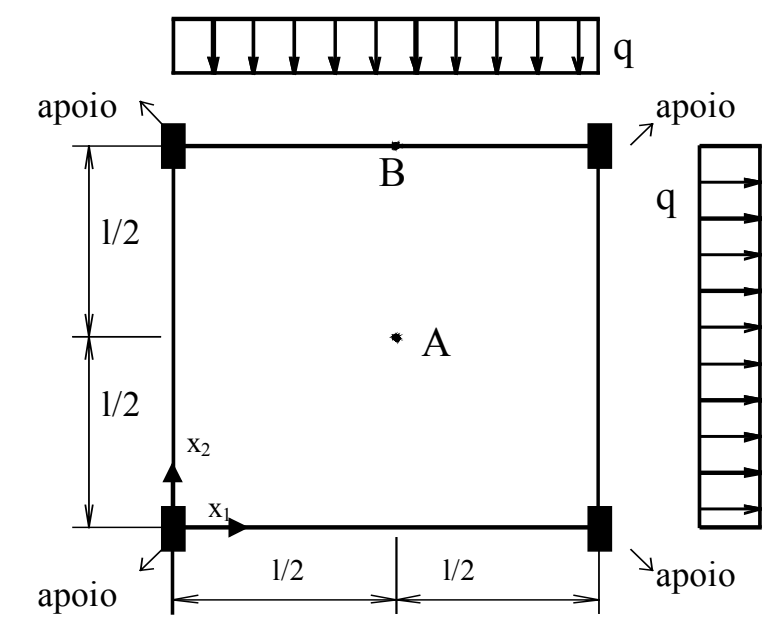

FIGURA 4.15 - Placa Quadrada Apoiada nos Quatro Cantos

Na tabela (4.2), estão indicados os resultados obtidos, juntamente com a solução analítica, dada por TIMOSHENKO (1959) e as soluções numéricas obtidas por PAIVA (1987), CALDERON (1991) e CHUEIRI (1994).

TABELA 4.2 - Resultados do exemplo 2

\begin{tabular}{|c|c|c|c|c|c|}
\hline \multirow[t]{2}{*}{ SOLUÇÕES } & \multirow[b]{2}{*}{$\mathbf{w} /\left(\mathbf{q} \mathbf{l}^{4} / \mathbf{D}\right)$} & \multirow{2}{*}{$\frac{\text { PONTO }}{\mathbf{M}_{11} / \mathbf{q} \mathbf{l}^{2}}$} & \multirow{2}{*}{$\frac{A}{M_{22} / \mathbf{q l}^{2}}$} & \multicolumn{2}{|c|}{ PONTO B } \\
\hline & & & & $\mathbf{w} /\left(\mathbf{q} \mathbf{l}^{4} / \mathbf{D}\right)$ & $\mathbf{M}_{11} /\left.\mathbf{q}\right|^{2}$ \\
\hline TIMOSHENKO & 0,0260 & 0,1090 & 0,1090 & $\ldots$ & 0,1404 \\
\hline PAIVA (40 elem.) & 0,0246 & 0,1100 & 0,1100 & 0,0163 & 0,1404 \\
\hline CALDERON (40 elem.) & 0,0271 & 0,1139 & 0,1139 & 0,0194 & \\
\hline CHUEIRI (16 elem, $\mathrm{a}=0,25)$ & 0,0260 & 0,1103 & 0,1103 & 0,0173 & 0,1437 \\
\hline \multicolumn{6}{|l|}{ PRESENTE ESTUDO } \\
\hline$(4$ elem, $\mathrm{a}=0,01)$ & 0,0263 & 0,1195 & 0,1195 & 0,0178 & 0,1469 \\
\hline (4 elem., $a=0,1)$ & 0,0257 & 0,1178 & 0,1178 & 0,0174 & 0,1438 \\
\hline$(4$ elem., $a=0,25)$ & 0,0253 & 0,1150 & 0,1150 & 0,0172 & 0,1438 \\
\hline (4 elem., $a=0,5)$ & 0,0255 & 0,1133 & 0,1133 & 0,0177 & 0,1498 \\
\hline$(16$ elem., $a=0,01)$ & 0,0260 & 0,1131 & 0,1131 & 0,0182 & 0,1358 \\
\hline$(16$ elem., $\mathrm{a}=0,1)$ & 0,0256 & 0,1125 & 0,1125 & 0,0178 & 0,1395 \\
\hline$(16$ elem., $a=0,25)$ & 0,0256 & 0,1122 & 0,1122 & 0,0178 & 0,1395 \\
\hline$(16$ elem., $a=0,5)$ & 0,0255 & 0,1120 & 0,1120 & 0,0178 & 0,1397 \\
\hline
\end{tabular}


Comparando-se os resultados obtidos com a discretização do contorno em 4 e 16 elementos, dados na tabela (4.2), observa-se uma melhora significativa nos resultados com o refinamento da malha. Os valores mais próximos da solução analítica, dada por TIMOSHENKO (1959), foram obtidos, no caso dos deslocamentos, para $\mathrm{a}=0,01 \mathrm{e}$, no caso dos momentos , para $\mathrm{a}=0,5$.

\subsubsection{Placa quadrada engastada nos quatro lados, com carregamento linearmente distribuído}

Nesse exemplo, utilizou-se somente pontos de colocação externos (ver figura 4.7.b), enquanto que o contorno foi discretizado em 16 elementos (ver figura 4.14), que define 36 pontos nodais. O valor de $\mathbf{a}_{1}$, dado em (4.27) e que determina a posição do ponto externo mais próximo ao contorno, será fixado e o valor de $\mathbf{a}_{2}$, que determina a posição do ponto mais afastado, será variável a fim de analisar a influência do mesmo no valor do deslocamento do ponto central $\mathbf{4}$ e dos momentos fletores dos pontos 1, 2, 3 e 4, indicados na figura (4.16).

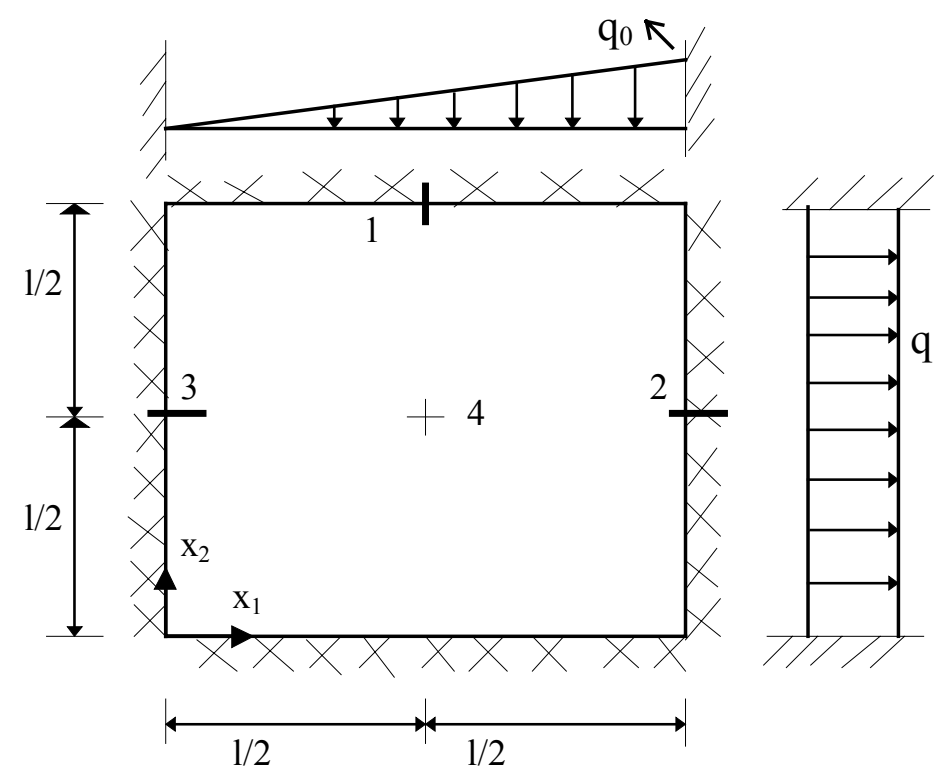

FIGURA 4.16 - Placa Engastada

Na tabela (4.3) estão indicados a solução analítica, dada por TIMOSHENKO (1959) e as soluções numéricas obtidas por PAIVA (1987) e CHUEIRI (1994). Os resultados obtidos com a formulação proposta nesse trabalho, estão indicados a seguir, na tabela (4.4) 
onde o valor de $\mathrm{a}_{1}$ foi fixado em 0,001 , variando-se o valor de $\mathrm{a}_{2}$. Como pode-se observar na tabela (4.4), os melhores resultados foram obtidos para $\mathrm{a}_{2}=0,01$.

TABELA 4.3 - Placa engastada com carga linearmente distribuída

\begin{tabular}{lccc}
\hline VALORES & TIMOSHENKO & $\begin{array}{c}\text { PAIVA } \\
(40 \text { elem. })\end{array}$ & $\begin{array}{c}\text { CHUEIRI } \\
(16 \text { elem., } a=0,25)\end{array}$ \\
\hline $\mathrm{w}_{4} /\left(\mathrm{q}_{0} 1^{4} / \mathrm{D}\right)$ & 0,00063 & 0,00063 & 0,00063 \\
\hline $\mathrm{M}_{11(1)} / \mathrm{q}_{0} 1^{2}$ & $-0,0077$ & $-0,0077$ & $-0,0077$ \\
$\mathrm{M}_{22(1)} / \mathrm{q}_{0} 1^{2}$ & $-0,0257$ & $-0,0259$ & $-0,0258$ \\
\hline $\mathrm{M}_{11(2)} / \mathrm{q}_{0} 1^{2}$ & $-0,0334$ & $-0,0338$ & $-0,0336$ \\
$\mathrm{M}_{22(2)} / \mathrm{q}_{0} 1^{2}$ & $-0,0100$ & $-0,0101$ & $-0,0100$ \\
\hline $\mathrm{M}_{11(3)} / \mathrm{q}_{0} 1^{2}$ & $-0,0179$ & $-0,0181$ & $-0,0180$ \\
$\mathrm{M}_{22(3)} / \mathrm{q}_{0} 1^{2}$ & $-0,0054$ & $-0,0054$ & $-0,0054$ \\
\hline $\mathrm{M}_{11(4)} / \mathrm{q}_{0} 1^{2}$ & 0,0115 & 0,0115 & 0,0115 \\
$\mathrm{M}_{22(4)} / \mathrm{q}_{0} 1^{2}$ & 0,0115 & 0,0115 & 0,0115 \\
\hline
\end{tabular}

TABELA 4.4 - Resultados do exemplo 3

\begin{tabular}{|c|c|c|c|c|c|c|c|}
\hline $\mathrm{valores}^{\mathrm{a}_{2}}$ & 0,01 & 0,1 & 0,25 & 0,5 & 0,7 & 1,0 & 1,5 \\
\hline $\mathrm{w}_{4} /\left(\mathrm{q}_{0} 1^{4} / \mathrm{D}\right) \times 10^{-4}$ & 6,33 & 6,33 & 6,33 & 6,33 & 6,33 & 6,33 & 6,33 \\
\hline $\mathrm{M}_{11(1)} / \mathrm{q}_{0} 1^{2} \times 10^{-3}$ & $-7,72$ & $-7,73$ & $-7,74$ & $-7,76$ & $-7,78$ & $-7,81$ & $-7,87$ \\
\hline $\mathrm{M}_{22(1)} / \mathrm{q}_{0} 1^{2} \times 10^{-2}$ & $-2,57$ & $-2,58$ & $-2,58$ & $-2,59$ & $-2,59$ & $-2,60$ & $-2,62$ \\
\hline $\mathrm{M}_{11(2)} / \mathrm{q}_{0} 1^{2} \times 10^{-2}$ & $-3,35$ & $-3,35$ & $-3,36$ & $-3,37$ & $-3,38$ & $-3,39$ & $-3,41$ \\
\hline $\mathrm{M}_{22(2)} / \mathrm{q}_{0} 1^{2} \times 10^{-2}$ & $-1,01$ & $-1,01$ & $-1,01$ & $-1,01$ & $-1,01$ & $-1,02$ & $-1,02$ \\
\hline $\mathrm{M}_{11(3)} / \mathrm{q}_{0} 1^{2} \times 10^{-2}$ & $-1,80$ & $-1,80$ & $-1,80$ & $-1,81$ & $-1,81$ & $-1,82$ & $-1,83$ \\
\hline $\mathrm{M}_{22(3)} / \mathrm{q}_{0} 1^{2} \times 10^{-3}$ & $-5,39$ & $-5,40$ & $-5,41$ & $-5,42$ & $-5,44$ & $-5,46$ & $-5,49$ \\
\hline $\mathrm{M}_{11(4)} / \mathrm{q}_{0} 1^{2} \times 10^{-2}$ & 1,15 & 1,15 & 1,15 & 1,15 & 1,15 & 1,15 & 1,15 \\
\hline $\mathrm{M}_{22(4)} / \mathrm{q}_{0} 1^{2} \times 10^{-2}$ & 1,15 & 1,15 & 1,15 & 1,15 & 1,15 & 1,15 & 1,15 \\
\hline
\end{tabular}

\subsubsection{Viga apoiada com momento aplicado nas extremidades}

A viga representada na figura (4.17), tem os lados de dimensão $\mathbf{2 b}$ apoiados, sendo os outros dois livres. O carregamento é definido pelo momento M, de valor $10000 \mathrm{Kgfxcm}$, aplicado nos lados menores (ver figura 4.17). A geometria da viga é dada por: $\mathrm{a}=75 \mathrm{~cm}, \mathrm{~b}=$ 
$10 \mathrm{~cm}$ e a espessura $\mathrm{t}=30 \mathrm{~cm}$. Utilizou-se um módulo de elasticidade $\mathrm{E}=300000 \mathrm{kgf} / \mathrm{cm}^{2} \mathrm{e}$ um coeficiente de poisson $v$ igual a 0 . O contorno foi discretizado em 4 elementos, que define 12 pontos nodais. O cálculo foi feito considerando-se dois pontos de colocação externos, sendo $a_{1}=0,01$ e $a_{2}=0,1$. Também foi experimentada uma segunda alternativa, para a qual foram adotandos um ponto de colocação externo $(a=0,1)$ e outro no contorno. As duas soluções obtidas foram praticamente idênticas.

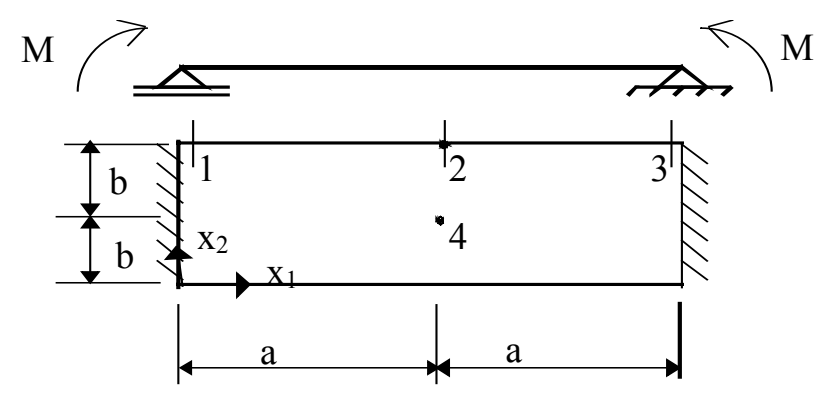

FIGURA 4.17 - Viga Simplesmente Apoiada

A seguir, na tabela (4.5), está indicada a solução obtida no segundo caso, onde os momentos são dados em Kgfxcm e os deslocamentos $\mathbf{w}$ em cm.

TABELA 4.5 - Resultados do exemplo 4

\begin{tabular}{llllllll}
\hline $\mathbf{M}_{11(1)}$ & $\mathbf{M}_{11(2)}$ & $\mathbf{M}_{11(3)}$ & $\mathbf{M}_{11(4)}$ & $\mathbf{M}_{22(4)}$ & $\mathbf{W}_{4}$ & $\mathbf{W}_{1}$ & $\mathbf{W}_{3}$ \\
\hline 10000 & 10000 & 10000 & 9999,8 & 0,16967 & 0,04167 & $-2 \times 10^{-9}$ & $-6 \times 10^{-9}$ \\
\hline
\end{tabular}

Como pode-se observar na tabela (4.5), obteve-se excelentes resultados, mesmo discretizando-se o contorno em apenas 4 elementos. 


\section{PROBLEMA DE PLACAS COM CAMPO DE MOMENTOS INICIAIS}

\subsection{Introdução}

Até aqui, desenvolveu-se o equacionamento da solução linear do problema de placas devido a um carregamento genérico transversal. Contudo, dentro da solução linear, deve-se considerar também a possibilidade de ocorrer momentos iniciais decorrentes de campos de deformações iniciais, tais como o efeito de temperatura ou retração. Além disso, os momentos iniciais devem ser considerados para a obtenção da solução não-linear do problema, como será visto no capítulo 7 .

\subsection{Equações Integrais com Campo de Momentos Iniciais}

\subsubsection{Equações Básicas}

Supondo-se que exista, além do carregamento transversal, um campo de deformações iniciais, o tensor de deformações pode ser escrito como:

$$
\varepsilon_{\mathrm{ij}}=\varepsilon_{\mathrm{ij}}^{\mathrm{e}}+\varepsilon_{\mathrm{ij}}^{0}
$$

onde: $\varepsilon_{\mathrm{ij}}$ é o campo de deformação total,

$\varepsilon_{\mathrm{ij}}^{\mathrm{e}}$ é a componente elástica devido ao carregamento, 
$\varepsilon_{\mathrm{ij}}^{0}$ é o campo de deformações iniciais.

Isolando-se a componente devido ao carregamento, tem-se:

$$
\varepsilon_{\mathrm{ij}}^{\mathrm{e}}=\varepsilon_{\mathrm{ij}}-\varepsilon_{\mathrm{ij}}^{0}
$$

Considerando-se a lei de Hooke, tem-se que:

$$
\sigma_{\mathrm{ij}}=\mathbf{2 G} \varepsilon_{\mathrm{ij}}^{\mathrm{e}}+\frac{\mathbf{2 G} v}{1-v} \varepsilon_{\mathrm{kk}}^{\mathrm{e}} \delta_{\mathrm{ij}} \quad(\mathrm{i}, \mathrm{j}, \mathrm{k}=1,2)
$$

Substituindo-se (5.2) em (5.3), obtém-se:

$$
\sigma_{\mathrm{ij}}=\sigma_{\mathrm{ij}}^{\mathrm{e}}-\sigma_{\mathrm{ij}}^{0}
$$

sendo:

$$
\sigma_{\mathrm{ij}}^{\mathrm{e}}=\mathbf{2} \mathbf{G} \varepsilon_{\mathrm{ij}}+\frac{\mathbf{2 G} v}{1-v} \varepsilon_{\mathbf{k} \mathbf{k}} \delta_{\mathrm{ij}} \text { as tensões devidas às deformações totais, }
$$

e

$$
\sigma_{\mathrm{ij}}^{0}=\mathbf{2} \mathbf{G} \varepsilon_{\mathrm{ij}}^{0}+\frac{\mathbf{2 G} v}{1-v} \varepsilon_{\mathbf{k k}}^{0} \delta_{\mathrm{ij}} \text { as tensões correspondentes às deformações iniciais. }
$$

Considerando-se que as deformações estão relacionadas às curvaturas $\mathbf{w}, \mathrm{ij}$, através da equação (2.4), obtém a expressão das tensões em função das curvaturas. Fazendo-se então, a integração destas tensões ao longo da espessura da placa, obtém-se o campo de momentos que atua na placa, que é dado por:

$$
\mathbf{M}_{\mathrm{ij}}=\mathbf{M}_{\mathrm{ij}}^{\mathrm{e}}-\mathbf{M}_{\mathrm{ij}}^{\mathbf{0}} \quad(\mathrm{i}, \mathrm{j}=1,2)
$$

onde: $\quad \mathbf{M}_{\mathrm{ij}}^{\mathrm{e}}$ são os momentos elásticos devido às tensões totais, e é dado por:

$$
\mathbf{M}_{\mathrm{ij}}^{\mathrm{e}}=-\mathbf{D}\left[v \mathbf{w},{ }_{k \mathrm{k}} \delta_{\mathrm{ij}}+(1-v) \mathbf{w},_{\mathrm{ij}}\right]
$$

$\mathbf{M}_{\mathrm{ij}}^{\mathbf{0}}$ é o campo de momentos iniciais, dado por: 


$$
M_{i j}^{0}=\int_{-t / 2}^{t / 2} \sigma_{i j}^{0} x_{3} d x_{3}
$$

A partir da equação de equilíbrio (2.13), podem-se obter os esforços cortantes, derivando-se a equação (5.5), isto é:

$$
\mathbf{Q}_{\mathbf{j}}=\mathbf{M}_{\mathrm{ij}, \mathrm{i}}=-\mathbf{D w},_{\mathbf{k k j}}-\mathbf{M}_{\mathrm{ij}, \mathrm{i}}^{\mathbf{0}} \quad(\mathrm{i}, \mathrm{j}, \mathrm{k}=1,2)
$$

Derivando-se a equação (5.8) e substituindo na equação de equilíbrio (2.11), chegase à equação diferencial de placas, envolvendo os momentos iniciais:

$$
\mathbf{w},{ }_{\mathrm{kkII}}=\frac{\mathbf{1}}{\mathbf{D}}\left(\mathbf{g}-\mathbf{M}_{\mathrm{ij}}^{\mathbf{0}} \boldsymbol{i}_{\mathrm{ij}}\right)
$$

\subsubsection{Equações Integrais para Deslocamentos}

Tais equações podem ser obtidas a partir do teorema de reciprocidade de Betti (equação 3.1), de forma análoga ao que foi feito no item (3.2). Assim, tem-se:

$$
\int_{\Omega}\left(\mathbf{M}_{\mathrm{ij}}^{*} \mathbf{w},{ }_{\mathrm{ij}}\right) \mathbf{d} \Omega=\int_{\Omega}\left(\mathbf{M}_{\mathrm{ij}}^{\mathrm{e}} \mathbf{w},{ }_{\mathrm{ij}}^{*}\right) \mathbf{d} \Omega \quad(\mathrm{i}, \mathrm{j}=1,2)
$$

Substituindo-se (5.5) em (5.10), obtém-se:

$$
\int_{\Omega}\left(\mathbf{M}_{\mathrm{ij}}^{*} \mathbf{w},,_{\mathrm{ij}}\right) \mathbf{d} \Omega=\int_{\Omega}\left(\mathbf{M}_{\mathrm{ij}}+\mathbf{M}_{\mathrm{ij}}^{0}\right) \mathbf{w},{ }_{\mathrm{ij}}^{*} \mathbf{d} \Omega \quad(\mathrm{i}, \mathrm{j}=1,2)
$$

Desenvolvendo-se os dois membros desta equação, através de integração por partes, semelhante ao que foi feito no item (3.2), obtém-se a equação integral do deslocamento de um ponto q do domínio da placa: 


$$
\begin{aligned}
& \mathbf{w}(\mathbf{q})+\int_{\Gamma}\left(\mathbf{V}_{\mathbf{n}}^{*}(\mathbf{q}, \mathbf{P}) \mathbf{w}(\mathbf{P})-\mathbf{M}_{\mathrm{nn}}^{*}(\mathbf{q}, \mathbf{P}) \frac{\partial \mathbf{w}}{\partial \mathbf{n}}(\mathbf{P})\right) \mathbf{d} \Gamma(\mathbf{P})+\sum_{\mathrm{i}=1}^{\mathbf{N}_{\mathrm{c}}} \mathbf{R}_{\mathrm{ci}}^{*}(\mathbf{q}, \mathbf{P}) \mathbf{w}_{\mathrm{ci}}(\mathbf{P})= \\
& =\int_{\Gamma}\left(\mathbf{V}_{\mathbf{n}}(\mathbf{P}) \mathbf{w}^{*}(\mathbf{q}, \mathbf{P})-\mathbf{M}_{\mathrm{nn}}(\mathbf{P}) \frac{\partial \mathbf{w}^{*}}{\partial \mathbf{n}}(\mathbf{q}, \mathbf{P})\right) \mathbf{d} \Gamma(\mathbf{P})+\sum_{\mathrm{i}=1}^{\mathbf{N}_{\mathrm{c}}} \mathbf{R}_{\mathrm{ci}}(\mathbf{P}) \mathbf{w}_{\mathrm{ci}}^{*}(\mathbf{q}, \mathbf{P})+ \\
& +\int_{\Omega_{\mathrm{g}}}\left(\mathbf{g}(\mathbf{p}) \mathbf{w}^{*}(\mathbf{q}, \mathbf{p})\right) \mathbf{d} \Omega_{\mathrm{g}}(\mathbf{p})-\int_{\Omega}\left(\mathbf{M}_{\mathrm{ij}}^{\mathbf{0}}(\mathbf{p}) \mathbf{w},{ }_{\mathrm{ij}}^{*}(\mathbf{q}, \mathbf{p})\right) \mathbf{d} \Omega(\mathbf{p})
\end{aligned}
$$

De forma análoga ao item (3.3), pode-se obter a equação integral de deslocamento para um ponto $\mathbf{Q}$ do contorno, que é dada por:

$$
\begin{aligned}
& \mathbf{K}(\mathbf{Q}) \mathbf{w}(\mathbf{Q})+\int_{\Gamma}\left(\mathbf{V}_{\mathbf{n}}^{*}(\mathbf{Q}, \mathbf{P}) \mathbf{w}(\mathbf{P})-\mathbf{M}_{\mathrm{nn}}^{*}(\mathbf{Q}, \mathbf{P}) \frac{\partial \mathbf{w}}{\partial \mathbf{n}}(\mathbf{P})\right) \mathbf{d} \Gamma(\mathbf{P})+ \\
& +\sum_{\mathrm{i}=1}^{\mathbf{N}_{\mathrm{c}}} \mathbf{R}_{\mathrm{ci}}^{*}(\mathbf{Q}, \mathbf{P}) \mathbf{w}_{\mathrm{ci}}(\mathbf{P})=\int_{\Gamma}\left(\mathbf{V}_{\mathrm{n}}(\mathbf{P}) \mathbf{w}^{*}(\mathbf{Q}, \mathbf{P})-\mathbf{M}_{\mathrm{nn}}(\mathbf{P}) \frac{\partial \mathbf{w}^{*}}{\partial \mathbf{n}}(\mathbf{Q}, \mathbf{P})\right) \mathbf{d} \Gamma(\mathbf{P})+ \\
& +\sum_{\mathrm{i}=1}^{\mathbf{N}_{\mathrm{c}}} \mathbf{R}_{\mathrm{ci}}(\mathbf{P}) \mathbf{w}_{\mathrm{ci}}^{*}(\mathbf{Q}, \mathbf{P})+\int_{\Omega_{\mathrm{g}}}\left(\mathbf{g}(\mathbf{p}) \mathbf{w}^{*}(\mathbf{Q}, \mathbf{p})\right) \mathbf{d} \Omega_{\mathrm{g}}(\mathbf{p})+ \\
& \quad-\int_{\Omega}\left(\mathbf{M}_{\mathrm{ij}}^{\mathbf{0}}(\mathbf{p}) \mathbf{w}_{\mathrm{ij}}^{*}(\mathbf{Q}, \mathbf{p})\right) \mathbf{d} \Omega(\mathbf{p})
\end{aligned}
$$

As equações (5.12) e (5.13) são iguais, respectivamente, às equações (3.19) e (3.67), a menos do termo que envolve os momentos iniciais $\mathbf{M}_{\mathrm{ij}}^{\mathbf{0}}$.

\subsubsection{Equações Integrais para Esforços nos Pontos Internos}

O cálculo dos momentos $\mathbf{M}_{\mathrm{ij}}$ para um ponto interno é feito a partir da equação (5.5), onde as curvaturas $\mathbf{w}{ }_{\mathrm{ij}}$, que aparecem na expressão de $\mathbf{M}_{\mathrm{ij}}^{\mathrm{e}}$, são obtidas derivando-se a equação (5.12) do deslocamento $\mathbf{w}(\mathbf{q})$ em relação as direções $\mathbf{x}_{\mathbf{1}}$ e $\mathbf{x}_{\mathbf{2}}$. Assim, a equação das curvaturas, é dada por:

$$
\frac{\partial^{2} \mathbf{w}(\mathbf{q})}{\partial \mathbf{x}_{\mathrm{i}} \partial \mathbf{x}_{\mathbf{j}}}=\left(\frac{\partial^{2} \mathbf{w}(\mathbf{q})}{\partial \mathbf{x}_{\mathrm{i}} \partial \mathbf{x}_{\mathbf{j}}}\right)^{\mathrm{e}}-\frac{\partial^{2}}{\partial \mathbf{x}_{\mathrm{i}} \partial \mathbf{x}_{\mathrm{j}}} \int_{\Omega}\left(\mathbf{M}_{\mathrm{kl}}^{\mathbf{0}}(\mathbf{p}) \mathbf{w},{ }_{\mathrm{kl}}^{*}(\mathbf{q}, \mathbf{p})\right) \mathbf{d} \Omega(\mathbf{p})
$$


onde: $\quad\left(\frac{\partial^{2} \mathbf{w}(\mathbf{q})}{\partial \mathbf{x}_{\mathbf{i}} \partial \mathbf{x}_{\mathbf{j}}}\right)^{\mathbf{e}}$ é a solução sem considerar momentos iniciais, dada por (4.48).

Denominado-se o segundo termo da equação (5.14), de $\mathrm{I}_{, \mathrm{ij}}^{0}(\mathbf{q})$, tem-se:

$$
\mathrm{I}_{, \mathrm{ij}}^{0}(\mathbf{q})=\frac{\partial^{2}}{\partial \mathbf{x}_{\mathrm{i}} \partial \mathbf{x}_{\mathrm{j}}} \int_{\Omega}\left(\mathbf{M}_{\mathrm{kl}}^{0}(\mathbf{p}) \mathbf{w},{ }_{\mathrm{kl}}^{*}(\mathbf{q}, \mathbf{p})\right) \mathbf{d} \Omega(\mathbf{p})
$$

onde o termo $\mathbf{w},{ }_{\mathbf{k l}}^{*}$ é dado por:

$$
\mathbf{w}_{\mathrm{kl}}^{*}(\mathbf{q}, \mathbf{P})=\frac{\partial^{2} \mathbf{w}^{*}}{\partial \mathbf{x}_{\mathrm{k}} \partial \mathbf{x}_{1}}(\mathbf{q}, \mathbf{P})=\frac{1}{4 \pi \mathrm{D}}\left(\mathbf{r},,_{\mathrm{k}} \mathbf{r},{ }_{1}+\delta_{\mathrm{kl}} \ln \mathbf{r}\right)
$$

A primeira derivada de $\mathbf{w},{ }_{\mathbf{k} l}^{*}$ em relação a $\mathbf{x}_{\mathbf{i}}$ é dada por:

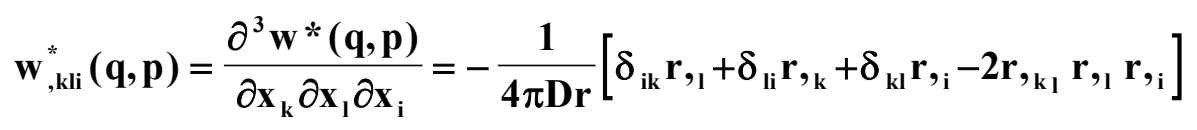

Como pode-se observar a expressão de $\mathrm{I}_{\mathbf{, j}}^{\mathbf{0}}(\mathbf{q})$ apresenta singularidades. A fim de eliminar tais singularidades, a mesma será calculada considerando o procedimento apresentado por MIKHLIN (1962), o qual também foi adotado por BUI (1978), TELLES \& BREBBIA (1979), RIBEIRO (1992) e CHUEIRI (1994).

Assim, a primeira derivada $\mathbf{w},{ }_{\mathbf{k l i}}^{*}$ pode ser calculada de forma normal no integrando, pois não ocorrem singularidades fortes. Portanto, pode-se escrever $\mathrm{I}_{, \mathrm{ij}}^{\mathbf{0}}$, na seguinte forma:

$$
\mathrm{I}_{, \mathrm{ij}}^{0}(\mathbf{q})=\frac{\partial}{\partial \mathbf{x}_{\mathrm{j}}} \int_{\Omega}\left(\mathbf{M}_{\mathrm{kl}}^{\mathbf{0}}(\mathbf{p}) \mathbf{w},{ }_{\mathrm{kli}}^{*}(\mathbf{q}, \mathbf{p})\right) \mathbf{d} \Omega(\mathbf{p})=\frac{\partial}{\partial \mathbf{x}_{\mathrm{j}}} \mathbf{V}(\mathbf{q})
$$

A integral $\mathbf{V}(\mathbf{q})$, indicada em (5.18), possui singularidade no domínio $\Omega$, portanto deve-se verificar a possibilidade de diferenciação do núcleo desta integral. Assim, supõe-se que, do domínio $\Omega$, é retirado um domínio circular $\Omega_{\mathrm{c}}$, onde está a singularidade, de pequeno raio $\varepsilon$ e com origem no ponto fonte $\mathbf{q}$, definindo-se o domínio $\Omega_{\varepsilon}=\Omega-\Omega_{\mathfrak{c}}$, conforme a figura (5.1). Assim, a integral $\mathbf{V}_{\varepsilon}(\mathbf{q})$ no domínio $\Omega_{\varepsilon}$ é dada por: 


$$
\begin{aligned}
\mathbf{V}_{\varepsilon}(\mathbf{q})= & \int_{\Omega_{\varepsilon}}\left(M_{\mathrm{kl}}^{0}(\mathbf{p}) \mathbf{w},{ }_{\mathrm{kli}}^{*}(\mathbf{q}, \mathbf{p})\right) \mathrm{d} \Omega_{\varepsilon}(\mathbf{p})=\int_{\Omega}\left(\mathbf{M}_{\mathrm{kl}}^{0}(\mathbf{p}) \mathbf{w},{ }_{\mathrm{kli}}^{*}(\mathbf{q}, \mathbf{p})\right) \mathrm{d} \Omega(\mathbf{p})+ \\
& -\int_{\Omega_{\mathrm{c}}}\left(\mathbf{M}_{\mathrm{kl}}^{0}(\mathbf{p}) \mathbf{w},{ }_{\mathrm{kli}}^{*}(\mathbf{q}, \mathbf{p})\right) \mathrm{d} \Omega_{\mathrm{c}}(\mathbf{p})=\mathrm{V}(\mathbf{q})-\mathbf{V}_{\mathrm{c}}(\mathbf{q})
\end{aligned}
$$

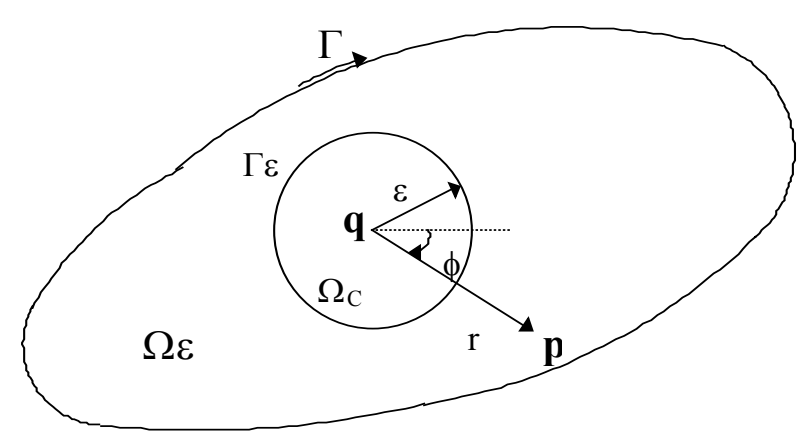

FIGURA 5.1 - Domínios $\Omega \varepsilon$ e $\Omega$ c

onde: $\mathbf{d} \Omega_{\mathrm{C}}=\operatorname{rdrd} \phi$,

$$
\mathbf{d} \Gamma_{\varepsilon}=\varepsilon \mathbf{d} \phi,
$$

Assume-se que a função $\mathbf{M}_{\mathbf{k l}}^{\mathbf{0}}(\mathbf{p})$ e suas primeiras e segundas derivadas são contínuas na vizinhança do ponto q. Assim, expandindo-se a mesma, em torno do ponto q, através da série de Taylor, tem-se que:

$$
\mathbf{M}_{\mathrm{kl}}^{\mathbf{0}}(\mathbf{p})=\mathbf{M}_{\mathrm{kl}}^{\mathbf{0}}(\mathbf{q})+\left[\mathbf{x}_{\mathbf{m}}(\mathbf{p})-\mathbf{x}_{\mathrm{m}}(\mathbf{q})\right] \mathbf{M}_{\mathrm{kl}}^{\mathbf{0}},_{\mathbf{m}}(\mathbf{q})+\ldots . \quad(\mathrm{k}, 1, \mathrm{~m}=1,2)
$$

Substituindo-se a equação (5.22) no termo $\mathbf{V}_{\mathbf{c}}(\mathbf{q})$ da equação (5.19), obtém-se:

$$
\begin{aligned}
\mathbf{V}_{\mathrm{c}}(\mathbf{q})= & \mathbf{M}_{\mathrm{kl}}^{0}(\mathbf{q}) \int_{\Omega_{\mathrm{C}}} \mathbf{w},{ }_{\mathrm{kli}}^{*}(\mathbf{q}, \mathbf{p}) \mathrm{d} \Omega_{\mathrm{C}}(\mathbf{p})+ \\
& +\mathbf{M}_{\mathrm{kl}}^{0},_{\mathrm{m}}(\mathbf{q}) \int_{\Omega_{\mathrm{C}}}\left[\mathbf{x}_{\mathrm{m}}(\mathbf{p})-\mathbf{x}_{\mathrm{m}}(\mathbf{q})\right] \mathbf{w},{ }_{\mathrm{kli}}^{*}(\mathbf{q}, \mathbf{p}) \mathrm{d} \Omega_{\mathrm{C}}(\mathbf{p})
\end{aligned}
$$

onde: $\mathbf{x}_{\mathbf{m}}(\mathbf{p})-\mathbf{x}_{\mathbf{m}}(\mathbf{q})=\mathbf{r} \cdot \mathbf{r},_{m}$ 
Substituindo-se em (5.23) o valor de $\mathbf{w},{ }_{\text {kli }}^{*}$, dado em (5.17), considerando-se as equações (5.20) e (5.24) e ainda, que no domínio $\Omega \mathbf{c}$, o valor de $\mathbf{r}$ é igual à $\varepsilon$ e que as derivadas dependem apenas do ângulo $\phi$, chega-se aos resultados:

$$
\int_{\Omega_{\mathrm{C}}} \mathbf{w},{ }_{\mathrm{kli}}^{*}(\mathbf{q}, \mathbf{p}) \mathrm{d} \Omega_{\mathrm{C}}(\mathbf{p})=\mathbf{0}
$$

e

$$
\int_{\Omega_{C}}\left[x_{m}(p)-x_{m}(q)\right] w,{ }_{k l i}^{*}(q, p) d \Omega_{C}(p)=-k \varepsilon^{2}
$$

onde: $\mathbf{K}=\frac{1}{16 \mathrm{D}}\left(\delta_{\mathrm{ik}} \delta_{\mathrm{Im}}+\delta_{\mathrm{il}} \delta_{\mathrm{km}}+\delta_{\mathrm{kl}} \delta_{\mathrm{im}}\right)$ é uma constante.

Logo, pode-se dizer que:

$$
\mathbf{V}_{\mathrm{c}}(\mathbf{q})=-K \varepsilon^{2} \mathbf{M}_{\mathrm{kl}}^{0} \boldsymbol{g}_{\mathrm{m}}(\mathbf{q})
$$

Desse modo, a derivada de $\mathbf{V}_{\mathbf{c}}(\mathbf{q})$ em relação a $\mathbf{x}_{\mathbf{j}}$ é dada por:

$$
\frac{\partial \mathbf{V}_{\mathbf{c}}(\mathbf{q})}{\partial \mathbf{x}_{\mathbf{j}}(\mathbf{q})}=-\mathbf{K} \varepsilon^{2} \mathbf{M}_{\mathrm{kl}}^{\mathbf{0}}, \mathbf{m j}_{\mathrm{j}}(\mathbf{q})
$$

Mas como $\varepsilon$ tende a zero, esta última também tende a zero quando toma-se o seu limite, e portanto, $I_{, j}^{0}(\mathbf{q})$ pode ser escrito na seguinte forma:

$$
\mathrm{I}_{, \mathrm{ij}}^{\mathbf{0}}(\mathbf{q})=\frac{\partial}{\partial \mathbf{x}_{\mathrm{j}}} \mathbf{V}_{\varepsilon}(\mathbf{q})=\lim _{\varepsilon \rightarrow 0}\left[\frac{\partial}{\partial \mathbf{x}_{\mathrm{j}}} \int_{\Omega_{\varepsilon}}\left(\mathbf{M}_{\mathrm{kl}}^{\mathbf{0}}(\mathbf{p}) \mathbf{w},{ }_{\mathrm{kli}}^{*}(\mathbf{q}, \mathbf{p})\right) \mathbf{d} \Omega_{\varepsilon}(\mathbf{p})\right]
$$

Pode-se demonstrar que, nesse caso, conforme MIKHLIN (1962) e BREBBIA et al (1984), aplicando-se a regra de Leibnitz para diferenciação de integrais, obtém-se:

$$
\mathrm{I}_{, \mathrm{ij}}^{\mathbf{0}}(\mathbf{q})=\int_{\Omega} \frac{\partial}{\partial \mathbf{x}_{\mathrm{j}}(\mathbf{q})} \mathbf{w},{ }_{\mathrm{kli}}^{*}(\mathbf{q}, \mathbf{p}) \mathbf{M}_{\mathrm{kl}}^{\mathbf{0}}(\mathbf{p}) \mathrm{d} \Omega(\mathbf{p})+
$$




$$
-M_{k l}^{0}(q) \int_{\Gamma_{\varepsilon}} w_{, k l i}^{*}(q, p) r,{ }_{j} d \Gamma_{\varepsilon}
$$

A primeira integral é interpretada no sentido do valor principal de Cauchy e considerando-se (5.21), a segunda integral pode ser escrita na seguinte forma:

$$
\int_{\Gamma_{\varepsilon}} \mathbf{w},{ }_{k l i}^{*}(q, p) r,{ }_{j} d \Gamma_{\varepsilon}=\int_{\phi=0}^{2 \pi} \varepsilon \cdot w,{ }_{k l i}^{*}(q, p) r,,_{j} d \phi
$$

Substituindo-se nesta última, o valor de $\mathbf{w},{ }_{\mathbf{k l i}}^{*}(\mathbf{q}, \mathbf{p})$, dado por (5.17), e fazendo-se $\mathbf{r}=\varepsilon$, tem-se que:

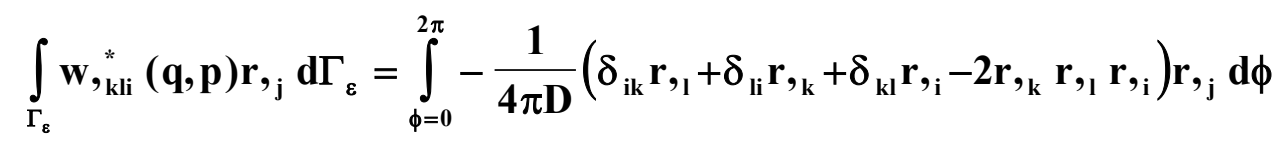

que resulta em:

$$
\int_{\Gamma_{\varepsilon}} \mathbf{w},{ }_{k l i}^{*}(q, p) r,{ }_{j} d \Gamma_{\varepsilon}=-\frac{1}{8 D}\left(\delta_{i k} \delta_{l j}+\delta_{l i} \delta_{k j}+\delta_{k l} \delta_{i j}\right)
$$

Desse modo, a equação (5.15), de $I_{, \mathbf{j}}^{\mathbf{0}} \mathbf{( q )}$, pode ser escrita da seguinte maneira:

$$
\mathrm{I}_{, \mathrm{ij}}^{0}(\mathbf{q})=\int_{\Omega}\left(\mathbf{M}_{\mathrm{kl}}^{0}(\mathbf{p}) \mathbf{w},{ }_{\mathrm{klij}}^{*}(\mathbf{q}, \mathbf{p})\right) \mathbf{d} \Omega(\mathbf{p})+\mathbf{M}_{\mathrm{kl}}^{0}(\mathbf{q}) \frac{1}{8 D}\left(\delta_{\mathrm{ik}} \delta_{\mathrm{lj}}+\delta_{\mathrm{li}} \delta_{\mathrm{kj}}+\delta_{\mathrm{kl}} \delta_{\mathrm{ij}}\right)
$$

ou:

$$
\mathrm{I}_{, \mathrm{ij}}^{0}(\mathbf{q})=\int_{\Omega}^{-} \overline{\mathrm{e}}_{\mathrm{ijkl}}^{*}(\mathbf{q}, \mathbf{p}) \mathbf{M}_{\mathrm{kl}}^{0}(\mathbf{p}) \mathrm{d} \Omega(\mathbf{p})+\overline{\mathbf{g}}_{\mathrm{ijkl}}(\mathbf{q}) \mathbf{M}_{\mathrm{kl}}^{0}(\mathbf{q})
$$

onde: $\quad \overline{\mathbf{e}}_{\mathrm{ij \textrm {jl }}}^{*}(\mathbf{q}, \mathbf{p})=\mathbf{w},{ }_{\mathrm{ijkl}}^{*}(\mathbf{q}, \mathbf{p})$

$$
\overline{\mathbf{g}}_{\mathrm{ijkl}}(q)=\frac{1}{\mathbf{8 D}}\left(\delta_{\mathrm{ik}} \delta_{\mathrm{lj}}+\delta_{\mathrm{li}} \delta_{\mathrm{kj}}+\delta_{\mathrm{kl}} \delta_{\mathrm{ij}}\right)
$$

Portanto, a equação das curvaturas nos pontos internos, é dada por: 


$$
\frac{\partial^{2} \mathbf{w}(\mathbf{q})}{\partial \mathbf{x}_{\mathrm{i}} \partial \mathbf{x}_{\mathrm{j}}}=\left(\frac{\partial^{2} \mathbf{w}(\mathbf{q})}{\partial \mathbf{x}_{\mathrm{i}} \partial \mathbf{x}_{\mathrm{j}}}\right)^{\mathrm{e}}-\int_{\Omega}^{-} \bar{e}_{\mathrm{ijkl}}^{*}(\mathbf{q}, \mathbf{p}) \mathbf{M}_{\mathrm{kl}}^{0}(\mathbf{p}) \mathbf{d} \Omega(\mathbf{p})-\overline{\mathbf{g}}_{\mathrm{ijkl}}(\mathbf{q}) \mathbf{M}_{\mathrm{kl}}^{\mathbf{0}}(\mathbf{q})
$$

A força cortante $\mathbf{Q}_{\beta}$, em relação a uma direção $\mathbf{x}_{\beta}(\beta=1,2)$, é calculada a partir da equação (5.8), onde o valor de $\mathbf{w}_{\mathbf{k k} \beta}$, é calculado derivando-se a equação (5.12) do deslocamento $\mathbf{w}(\mathbf{q})$ de um ponto interno:

$$
\begin{aligned}
\frac{\partial}{\partial \mathbf{x}_{\beta}(\mathbf{q})}\left(\frac{\partial^{2} \mathbf{w}(\mathbf{q})}{\partial \mathbf{x}_{\mathbf{k}} \partial \mathbf{x}_{\mathbf{k}}}\right)= & {\left[\frac{\partial}{\partial \mathbf{x}_{\beta}(\mathbf{q})}\left(\frac{\partial^{2} \mathbf{w}(\mathbf{q})}{\partial \mathbf{x}_{\mathbf{k}} \partial \mathbf{x}_{\mathrm{k}}}\right)\right]^{\mathrm{e}}+} \\
& -\frac{\partial}{\partial \mathbf{x}_{\beta}(\mathbf{q})} \int_{\Omega}\left(\frac{\partial^{2} \mathbf{w}_{\mathbf{k l}}^{*}(\mathbf{q}, \mathbf{p})}{\partial \mathbf{x}_{\mathrm{m}} \partial \mathbf{x}_{\mathbf{m}}} \mathbf{M}_{\mathbf{k l}}^{0}(\mathbf{p})\right) \mathbf{d} \Omega(\mathbf{p})
\end{aligned}
$$

onde $\left[\frac{\partial}{\partial \mathbf{x}_{\beta}(\mathbf{q})}\left(\frac{\partial^{2} \mathbf{w}(\mathbf{q})}{\partial \mathbf{x}_{\mathbf{k}} \partial \mathbf{x}_{\mathbf{k}}}\right)\right]^{\mathrm{e}}$ representa a solução sem considerar momentos iniciais, dada por (4.60).

Denominando-se o segundo termo da equação (5.37), de $\mathrm{I}_{\beta}^{0}$, tem-se:

$$
\mathrm{I}_{, \beta}^{0}=\frac{\partial}{\partial \mathbf{x}_{\beta}(\mathbf{q})} \int_{\Omega}\left(\frac{\partial^{2} \mathbf{w}_{\mathrm{kl}}^{*}(\mathbf{q}, \mathbf{p})}{\partial \mathbf{x}_{\mathbf{m}} \partial \mathbf{x}_{\mathbf{m}}} \mathbf{M}_{\mathbf{k l}}^{0}(\mathbf{p})\right) \mathbf{d} \Omega(\mathbf{p})
$$

Porém, considerando-se a equação (5.33), obtém-se:

$$
\begin{aligned}
I_{, \mathrm{mm}}^{0}=\int_{\Omega}\left(M_{\mathrm{kl}}^{0}(\mathbf{p}) \mathbf{w},,_{\mathrm{klmm}}^{*}(\mathbf{q}, \mathbf{p})\right) \mathbf{d} \Omega(\mathbf{p})=\int_{\Omega}\left(\mathbf{M}_{\mathrm{kl}}^{\mathbf{0}}(\mathbf{p}) \mathbf{w},{ }_{\mathrm{klmm}}^{*}(\mathbf{q}, \mathbf{p})\right) \mathbf{d} \Omega(\mathbf{p})+ \\
+\mathbf{M}_{\mathrm{kl}}^{\mathbf{0}}(\mathbf{q}) \frac{1}{8 \mathrm{D}}\left(4 \delta_{\mathrm{kl}}\right)=\int_{\Omega}\left(\mathbf{M}_{\mathrm{kl}}^{0}(\mathbf{p}) \mathbf{w},{ }_{\mathrm{klmm}}^{*}(\mathbf{q}, \mathbf{p})\right) \mathbf{d} \Omega(\mathbf{p})+ \\
+\frac{1}{2 \mathrm{D}}\left(\mathbf{M}_{11}^{0}+\mathbf{M}_{22}^{0}\right)
\end{aligned}
$$

Desse modo, substituindo-se a equação (5.39) em (5.38), pode-se escrever: 


$$
\begin{gathered}
I_{, \beta}^{0}=\frac{\partial}{\partial \mathbf{x}_{\beta}(\mathbf{q})} \int_{\Omega}\left(\mathbf{M}_{\mathbf{k l}}^{0}(\mathbf{p}) \mathbf{w}_{\mathbf{k}_{\mathrm{klmm}}}^{*}(\mathbf{q}, \mathbf{p})\right) \mathbf{d} \Omega(\mathbf{p})+ \\
+\frac{\partial}{\partial \mathbf{x}_{\beta}(\mathbf{q})}\left[\frac{1}{2 \mathrm{D}}\left(\mathbf{M}_{11}^{0}+\mathbf{M}_{22}^{0}\right)\right]
\end{gathered}
$$

Nesta última, a integral $\mathbf{V}^{\prime}(\mathbf{q})=\int_{\Omega}\left(\frac{\partial^{2} \mathbf{w},{ }_{\mathbf{k l}}^{*}(\mathbf{q}, \mathbf{p})}{\partial \mathbf{x}_{\mathbf{m}} \partial \mathbf{x}_{\mathbf{m}}} \mathbf{M}_{\mathbf{k l}}^{\mathbf{0}}(\mathbf{p})\right) \mathbf{d} \Omega(\mathbf{p})$ é calculada de maneira análoga ao que foi feito para $\mathbf{V}(\mathbf{q})$ (5.18). Desse modo, denominando-se $\mathbf{V}_{\mathbf{c}}^{\prime}(\mathbf{q})$ a parcela da integral $\mathbf{V}^{\prime}(\mathbf{q})$ no domínio $\Omega \mathbf{c}$, onde há a singularidade, esta pode ser escrita como:

$$
\begin{aligned}
\mathbf{V}_{\mathrm{c}}^{\prime}(\mathbf{q})= & \mathbf{M}_{\mathrm{kl}}^{0}(\mathbf{q}) \int_{\Omega_{\mathrm{C}}} \mathbf{w},{ }_{\mathrm{mmkl}}^{*}(\mathbf{q}, \mathbf{p}) \mathrm{d} \Omega_{\mathrm{C}}(\mathbf{p})+ \\
& +\mathbf{M}_{\mathrm{kl}}^{0},_{\mathrm{j}}(\mathbf{q}) \int_{\Omega_{\mathrm{C}}}\left[\mathbf{x}_{\mathrm{j}}(\mathbf{p})-\mathbf{x}_{\mathrm{j}}(\mathbf{q})\right] \mathbf{w},{ }_{\mathrm{mmkl}}^{*}(\mathbf{q}, \mathbf{p}) \mathrm{d} \Omega_{\mathrm{C}}(\mathbf{p})
\end{aligned}
$$

Substituindo-se nesta última o valor de $\mathbf{w},{ }_{\text {mmkl }}^{*}$, considerando-se as equações (5.20) e (5.24) e ainda, que o valor de $\mathbf{r}$ é igual a $\varepsilon$ no domínio $\Omega_{c}$, e que as funções envolvidas dependem apenas do ângulo $\phi$, chega-se aos resultados:

$$
\int_{\Omega_{\mathrm{C}}} \mathbf{w},{ }_{\mathrm{mmkl}}^{*}(\mathbf{q}, \mathbf{p}) \mathrm{d} \Omega_{\mathrm{C}}(\mathbf{p})=\mathbf{0}
$$

e

$$
\int_{\Omega_{\mathrm{C}}}\left[\mathbf{x}_{\mathbf{j}}(\mathbf{p})-\mathbf{x}_{\mathbf{j}}(\mathbf{q})\right] \mathbf{w},{ }_{\mathrm{mmkl}}^{*}(\mathbf{q}, \mathbf{p}) \mathrm{d} \Omega_{\mathrm{C}}(\mathbf{p})=\mathbf{0}
$$

Logo, pode-se escrever que:

$$
\frac{\partial \mathbf{V}^{\prime}(\mathbf{q})}{\partial \mathbf{x}_{\beta}(\mathbf{q})}=\lim _{\varepsilon \rightarrow 0}\left[\frac{\partial}{\partial \mathbf{x}_{\beta}(\mathbf{q})} \int_{\Omega_{\varepsilon}} \mathbf{w},{ }_{\text {mmkl }}^{*}(\mathbf{q}, \mathbf{p}) \mathbf{d M}_{\mathbf{k l}}^{0}(\mathbf{p}) \Omega_{\varepsilon}(\mathbf{p})\right]
$$

Aplicando-se a regra de Leibnitz para diferenciação de integrais, obtém-se:

$$
\frac{\partial V^{\prime}(\mathbf{q})}{\partial \mathbf{x}_{\beta}(\mathbf{q})}=\int_{\Omega} \frac{\partial \mathbf{w}_{\text {mmkl }}^{*}}{\partial \mathbf{x}_{\beta}(\mathbf{q})}(\mathbf{q}, \mathbf{p}) \mathbf{M}_{\mathrm{kl}}^{\mathbf{0}}(\mathbf{p}) \mathrm{d} \Omega(\mathbf{p})=\int_{\Omega} \mathbf{e}_{\beta \mathrm{kl}}^{*}(\mathbf{q}, \mathbf{p}) \mathbf{M}_{\mathrm{kl}}^{0}(\mathbf{p}) \mathrm{d} \Omega(\mathbf{p})
$$


Logo, substituindo-se (5.45) em (5.40), tem-se que:

$$
\mathrm{I}_{, \beta}^{0}=\int_{\Omega} \mathbf{e}_{\beta \mathrm{kl}}^{*}(\mathbf{q}, \mathbf{p}) \mathbf{M}_{\mathrm{kl}}^{0}(\mathbf{p}) \mathrm{d} \Omega(\mathbf{p})+\overline{\mathbf{q}}_{\beta}^{0}(\mathbf{q})
$$

onde: $\quad \overline{\mathbf{q}}_{\beta}^{0}(\mathbf{q})=\frac{1}{2 D} \frac{\partial}{\partial \mathbf{x}_{\beta}(\mathbf{q})}\left(\mathbf{M}_{11}^{0}(\mathbf{q})+\mathbf{M}_{22}^{0}(\mathbf{q})\right)$

$$
\mathbf{e}_{\beta \mathbf{k l}}^{*}(\mathbf{q}, \mathbf{p})=\frac{\partial \mathbf{w},{ }_{\text {mmkl }}^{*}}{\partial \mathbf{x}_{\beta}(\mathbf{q})}(\mathbf{q}, \mathbf{p})
$$

Assim, a equação da derivada das curvaturas é dada por:

$$
\begin{aligned}
\frac{\partial}{\partial \mathbf{x}_{\beta}(\mathbf{q})}\left(\frac{\partial^{2} \mathbf{w}(\mathbf{q})}{\partial \mathbf{x}_{\mathbf{k}} \partial \mathbf{x}_{\mathrm{k}}}\right) & =\left[\frac{\partial}{\partial \mathbf{x}_{\beta}(\mathbf{q})}\left(\frac{\partial^{2} \mathbf{w}(\mathbf{q})}{\partial \mathbf{x}_{\mathbf{k}} \partial \mathbf{x}_{\mathrm{k}}}\right)\right]^{\mathrm{e}}+ \\
& -\int_{\Omega} \mathbf{e}_{\beta \mathrm{kl}}^{*}(\mathbf{q}, \mathbf{p}) \mathbf{M}_{\mathrm{kl}}^{0}(\mathbf{p}) \mathbf{d} \Omega(\mathbf{p})-\overline{\mathbf{q}}_{\beta}^{\mathbf{0}}(\mathbf{q})
\end{aligned}
$$

\subsection{Equações Algébricas}

De maneira análoga ao que foi feito no capítulo (4), pode-se transformar as equações integrais obtidas no item (5.2) em equações algébricas, através da discretização do contorno em elementos e do domínio em células, nas quais será aproximado o campo de momentos iniciais.

\subsubsection{Discretização do Domínio da Placa}

As integrais de domínio envolvendo o campo de momentos iniciais, que aparecem nas equações de deslocamento (5.12) e (5.13), das curvaturas (5.36) e da derivada das curvaturas (5.49), podem ser calculadas numericamente, através da divisão do domínio em pequenas regiões, ou células (Figura 5.2), nas quais os momentos iniciais são aproximados por funções interpoladoras lineares $\Psi(\mathbf{p})$. Obtêm-se, assim, as integrais das funções envolvidas sobre cada elemento de área. Neste trabalho, adotam-se células de forma 
triangular (Figura 5.2), que são definidas, de modo a envolver todos os pontos do contorno e internos.

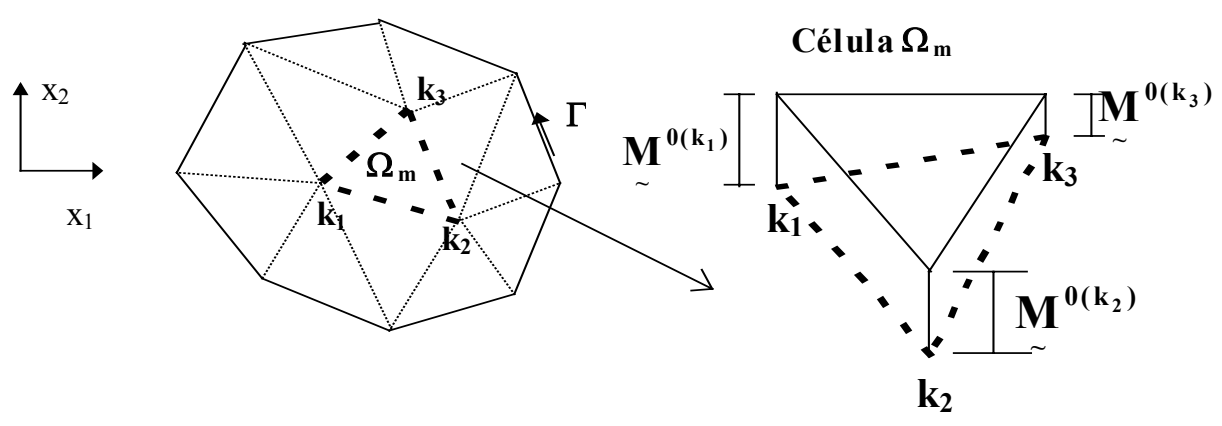

FIGURA 5.2 - Divisão do Domínio da Placa em Células Triangulares e a Variação dos Momentos Iniciais em uma Célula

\subsubsection{Aproximação da geometria da célula e do Campo de Momentos Iniciais}

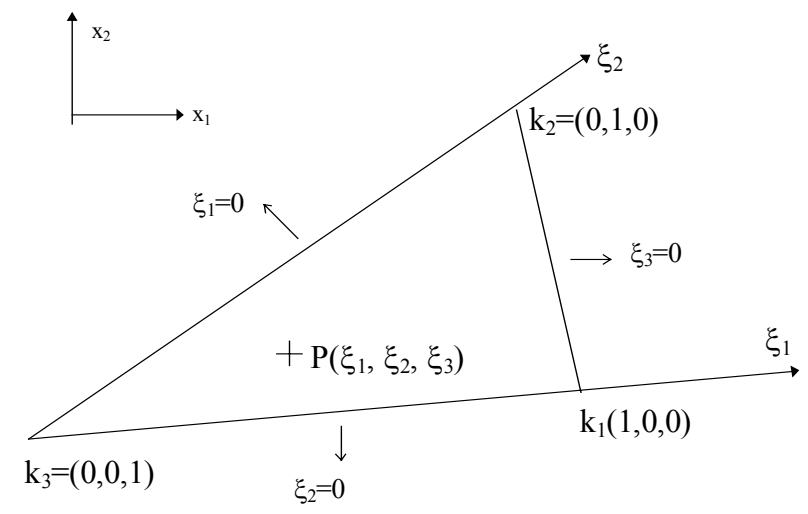

FIGURA 5.3 - Célula Triangular com Sistema de Coordenadas Homogêneas

A geometria da célula é aproximada por uma função de interpolação linear $\psi_{\mathrm{g}}$, que é função de coordenadas homogêneas. Os nós $\mathbf{k}_{\mathbf{1}}, \mathbf{k}_{\mathbf{2}}, \mathbf{k}_{\mathbf{3}}$ de uma célula triangular serão definidos por suas coordenadas homogêneas, como é mostrado na figura (5.3). Desse modo, as coordenadas cartesianas $\mathbf{X}_{1}^{\mathbf{p}}$ e $\mathbf{X}_{2}^{\mathbf{p}}$ de um ponto $\mathbf{p}$ qualquer da célula, podem ser definidas em função das coordenadas dos vértices $\mathbf{k}_{\mathbf{1}}, \mathbf{k}_{\mathbf{2}}, \mathbf{k}_{\mathbf{3}}$ da mesma, através de: 


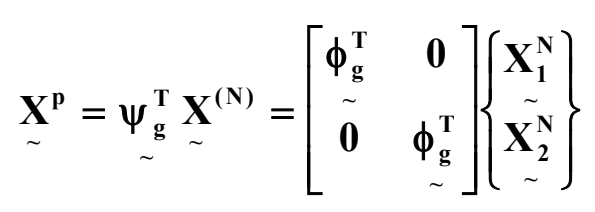

onde: $\quad \underset{\sim}{\mathbf{X}^{\mathbf{p}}}=\left\{\begin{array}{l}\mathbf{x}_{1}^{\mathbf{p}} \\ \mathbf{x}_{2}^{\mathbf{p}}\end{array}\right\}$ é o vetor de coordenadas cartesianas do ponto $\mathbf{p}$

$$
\begin{aligned}
& \underset{\sim}{\phi_{\mathbf{g}}}=\left\{\begin{array}{l}
\xi_{1}^{\mathbf{p}} \\
\xi_{2}^{\mathbf{p}} \\
\xi_{3}^{\mathbf{p}}
\end{array}\right\} \text { é o vetor das coordenadas homogêneas do ponto } \mathbf{p}, \\
& \underset{\sim}{\mathbf{X}_{\mathbf{i}}^{\mathbf{N}}}=\left\{\begin{array}{l}
\mathbf{x}_{\mathbf{i}}^{\mathbf{k}_{1}} \\
\mathbf{x}_{\mathbf{i}}^{\mathbf{k}_{2}} \\
\mathbf{x}_{\mathbf{i}}^{\mathbf{k}_{3}}
\end{array}\right\} \text { é o vetor de coordenadas cartesianas nodais, na direção } \mathbf{i}
\end{aligned}
$$

Ou na forma explícita:

$$
\mathbf{X}_{\mathbf{i}}^{\mathbf{p}}=\xi_{1}^{\mathbf{p}} \mathbf{X}_{\mathbf{i}}^{\mathbf{k}_{1}}+\xi_{2}^{\mathbf{p}} \mathbf{X}_{\mathbf{i}}^{\mathbf{k}_{2}}+\xi_{3}^{\mathbf{p}} \mathbf{X}_{\mathbf{i}}^{\mathbf{k}_{3}}
$$

onde: $\quad \xi_{\alpha}^{\mathbf{p}}=\frac{\mathbf{1}}{\mathbf{2 A}}\left(\mathbf{2}_{\mathbf{0}}^{\alpha}+\mathbf{b}^{\alpha} \mathbf{X}_{1}^{\mathbf{p}}+\mathbf{a}^{\alpha} \mathbf{X}_{2}^{\mathbf{p}}\right) \quad \alpha=1,2,3 ; \mathrm{j}=2,3,1 ; \mathrm{k}=3,1,2$

$$
\begin{aligned}
& \mathbf{a}^{\alpha}=\mathbf{X}_{1}^{\mathbf{k}}-\mathbf{X}_{1}^{\mathrm{j}} \\
& \mathbf{b}^{\alpha}=\mathbf{X}_{2}^{\mathrm{k}}-\mathbf{X}_{2}^{\mathbf{j}} \\
& \mathbf{2} \mathbf{A}_{0}^{\alpha}=\mathbf{X}_{1}^{\mathbf{j}} \mathbf{X}_{2}^{\mathbf{k}}-\mathbf{X}_{1}^{\mathbf{k}} \mathbf{X}_{2}^{\mathbf{j}}
\end{aligned}
$$

A é a área do triângulo, que é dada por:

$$
A=\frac{1}{2}\left(b^{1} \mathbf{a}^{2}-\mathbf{b}^{2} \mathbf{a}^{1}\right)
$$

O campo de momentos iniciais $\mathbf{M}_{\mathrm{ij}}^{\mathbf{0}}$ sobre cada célula será aproximado por uma função de interpolação linear $\psi$. Desse modo, os momentos iniciais $\underset{\sim}{\mathbf{M}_{\mathbf{i j}}^{\mathbf{0}}}(\mathbf{p})$, em um ponto $\mathbf{p}$ qualquer da célula, são dados por:

$$
\underset{\sim \mathrm{ij}}{\mathbf{M}^{0}}(\mathrm{p})={\underset{\sim}{\Psi}}^{\mathbf{T}}(\mathbf{p}) \underset{\sim}{\mathbf{M}^{0(N)}}
$$


sendo: $\quad \underset{\sim \mathrm{ij}}{\mathbf{M}_{\mathrm{ij}}^{0}}(\mathbf{p})=\left\{\begin{array}{l}\mathbf{M}_{11}^{0}(\mathbf{p}) \\ \mathbf{M}_{12}^{0}(\mathbf{p}) \\ \mathbf{M}_{22}^{0}(\mathbf{p})\end{array}\right\}$ o vetor que contém os momentos iniciais no ponto $\mathbf{p}$,

$$
\underset{\sim}{\Psi^{T}}=\left[\begin{array}{ccccccccc}
\xi_{1}^{p} & \mathbf{0} & \mathbf{0} & \xi_{2}^{\mathbf{p}} & \mathbf{0} & \mathbf{0} & \xi_{3}^{\mathrm{p}} & \mathbf{0} & \mathbf{0} \\
\mathbf{0} & \xi_{1}^{\mathbf{p}} & \mathbf{0} & \mathbf{0} & \xi_{2}^{\mathbf{p}} & \mathbf{0} & \mathbf{0} & \xi_{3}^{\mathbf{p}} & \mathbf{0} \\
\mathbf{0} & \mathbf{0} & \xi_{1}^{\mathbf{p}} & \mathbf{0} & \mathbf{0} & \xi_{2}^{\mathrm{p}} & \mathbf{0} & \mathbf{0} & \xi_{3}^{\mathrm{p}}
\end{array}\right]
$$

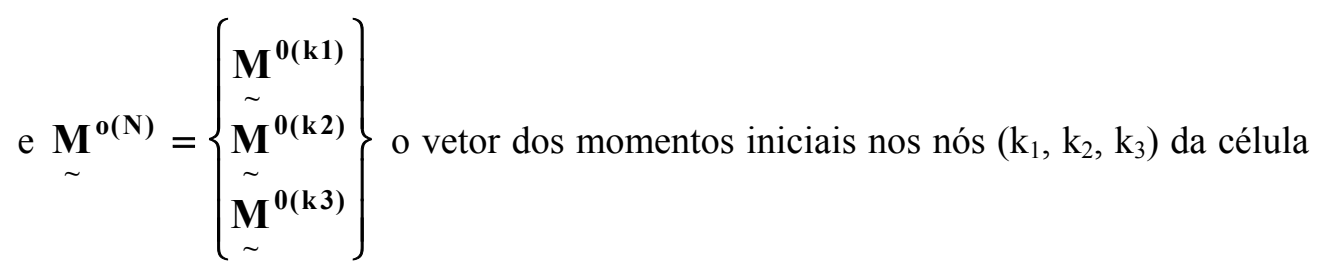
a qual o ponto $\mathbf{p}$ pertence.

\subsubsection{Integração sobre as Células}

A integração sobre cada célula pode ser feita através da fórmula de quadratura de Gauss para domínio triangular, utilizando-se as coordenadas homogêneas. Nesse caso, o Jacobiano da transformação de coordenadas é igual ao dobro da área do triângulo da célula. Porém, devido à natureza das funções envolvidas, há necessidade de se utilizar um grande número de pontos de integração, para se obter uma precisão aceitável, dependendo da posição do ponto de carregamento em relação a célula. Por esta razão, adota-se, neste trabalho, um esquema semi-analítico de integração, utilizado por TELLES \& BREBBIA (1979) e VENTURINI (1982), o qual apresenta melhores resultados.

Para a implementação desse esquema, adota-se um sistema de coordenadas cilíndricas $(\mathbf{r}, \theta)$, centrado no ponto de carregamento $\mathbf{q}$, conforme a figura (5.4), que torna possível calcular analiticamente a integral sobre a coordenada $\mathbf{r}$. Em relação a esse sistema, a coordenada adimensional $\xi_{\alpha}^{\mathbf{P}}$, que aparece na função de interpolação ${\underset{\sim}{\Psi}}^{\mathrm{T}}$, dada pela equação (5.58), é dada por:

$$
\xi_{\alpha}^{\mathbf{p}}=\xi_{\alpha}^{\mathbf{q}}+\frac{\mathbf{r}}{\mathbf{2 A}}\left(\mathbf{b}^{\alpha} \cos \theta+\mathbf{a}^{\alpha} \operatorname{sen} \theta\right)
$$

e $\xi_{\alpha}^{\mathbf{q}}$ é obtido da equação (5.52), para o ponto $\mathbf{q}$ e $\mathbf{a}^{\alpha}, \mathbf{b}^{\alpha}$ e $\mathbf{A}$ são dados respectivamente, por (5.53), (5.54) e (5.56). 


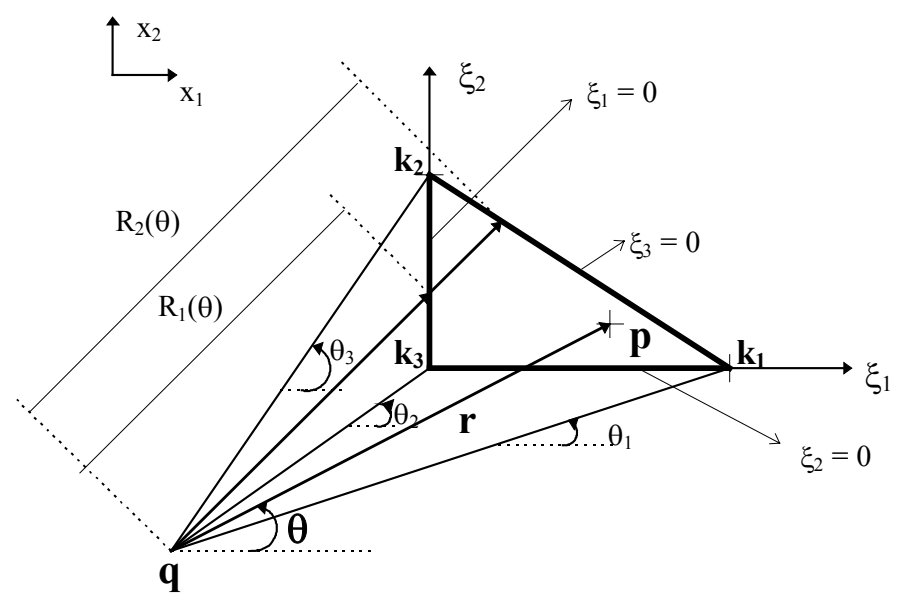

FIGURA 5.4 - Sistema de Coordenadas Cilíndricas

Quando o ponto q coincidir com um dos cantos $\alpha$ do triângulo, tem-se que:

$$
\xi_{\alpha}^{q}=\left\{\begin{array}{lll}
1 & \text { se } & \alpha=\mathbf{q} \\
0 & \text { se } & \alpha \neq \mathbf{q}
\end{array}\right.
$$

Na figura (5.4), quando o ponto $\mathbf{p}$ estiver no lado do triângulo onde $\xi_{1}=\mathbf{0}$ ou no lado onde $\xi_{2}=\mathbf{0}$, o valor da distância $\mathbf{r}$, entre $\mathbf{q}$ e $\mathbf{p}$, será dada por $\mathbf{R}_{\mathbf{1}}(\theta)$ e quando o mesmo estiver no lado onde $\xi_{3}=\mathbf{0}$, tem-se $\mathbf{r}=\mathbf{R}_{\mathbf{2}}(\theta)$. Assim, usando-se esses valores particulares de $\xi_{\alpha}^{\mathbf{p}}$ na equação (5.60), obtém-se:

$$
\mathbf{R}_{\mathbf{j}}(\theta)=-\frac{2 A \xi_{\alpha}^{q}}{\left(\mathbf{b}^{\alpha} \cos \theta+\mathbf{a}^{\alpha} \operatorname{sen} \theta\right)}
$$

onde: $\mathrm{j}=2$, para $\alpha=3 \mathrm{e} \mathrm{j}=1$, para $\alpha=1,2$.

Assim, utilizando-se esse esquema, as integrais sobre as células são calculadas numericamente em relação a $\theta$, empregando-se a fórmula de quadratura de Gauss. CHAVES (1997) transforma as integrais obtidas utilizando-se esse esquema, que são escritas em função de $\theta$, em integrais sobre o contorno da célula, através da seguinte transformação de coordenadas: $\mathbf{d} \theta=\frac{\mathbf{r}_{i_{i}} \mathbf{n}_{\mathbf{i}}}{\mathbf{r}} \mathbf{d} \Gamma$. É interessante essa transformação, pois ela permite a 
implementação da técnica de sub-elementos, descrita no item (4.7.2), aumentando-se, desse modo, a precisão do cálculo.

Os itens (5.4.1), (5.4.2) e (5.4.3) discutem a possibilidade de integração numérica das integrais que envolvem os momentos iniciais, que aparecem nas equações integrais dos itens (5.2.2) e (5.2.3)

\subsection{Integração Numérica Sobre as Células}

5.4.1 Integração sobre as células referente à equação do deslocamento w de um ponto do contorno ou do domínio

A integral (5.13) do deslocamento de um ponto do contorno, pode ser representada matricialmente, de forma semelhante à equação (4.13), ou seja:

$$
\begin{aligned}
& \underset{\sim}{\mathbf{K}}(\mathbf{Q}) \underset{\sim}{\mathbf{w}}(\mathbf{Q})+\int_{\Gamma} \mathbf{p}_{\sim}^{*}(\mathbf{Q}, \mathbf{P}) \underset{\sim}{\mathbf{u}}(\mathbf{P}) \mathrm{d} \Gamma(\mathbf{P})+\sum_{\mathrm{i}=1}^{\mathbf{N}_{\mathrm{c}}} \mathbf{R}_{\mathrm{ci}}^{*}(\mathbf{Q}, \mathbf{P}) \mathbf{w}_{\mathrm{ci}}(\mathbf{P})=
\end{aligned}
$$

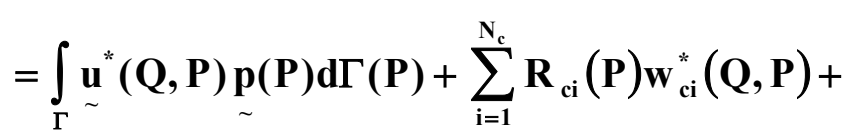

$$
\begin{aligned}
& -\int_{\Omega} \mathbf{k}_{\sim}^{*}(\mathbf{Q}, \mathbf{p}) \underset{\sim}{\mathbf{M}_{\mathrm{ij}}^{\mathbf{0}}}(\mathbf{p}) \mathrm{d} \Omega(\mathbf{p})
\end{aligned}
$$

onde: $\underset{\sim}{\mathbf{k}}(\mathbf{Q}, \mathbf{p})=\left\{\frac{\partial^{2} \mathbf{w}^{*}}{\partial \mathbf{x}_{1}^{2}}(\mathbf{Q}, \mathbf{p}) \quad 2 \frac{\partial^{2} \mathbf{w}^{*}}{\partial \mathbf{x}_{1} \partial \mathbf{x}_{2}}(\mathbf{Q}, \mathbf{p}) \frac{\partial^{2} \mathbf{w}^{*}}{\partial \mathbf{x}_{2}^{2}}(\mathbf{Q}, \mathbf{p})\right\}$,

$\mathbf{M}_{\mathrm{ij}}^{\mathbf{0}}(\mathbf{p})$ é dado pela equação (5.57) e os outros termos estão definidos em (4.13).

Discretizando-se o contorno da placa em $\mathbf{N}_{\mathrm{e}}$ elementos e aproximando-se os valores dos deslocamentos e esforços nos mesmos, como foi mostrado no item (4.2.3), dividindo-se o domínio em $\mathbf{N}_{\mathrm{ce}}$ células e aproximando-se os momentos iniciais nas mesmas (equação 5.57), pode-se escrever a equação (5.62) na seguinte forma:

$$
\mathbf{K}(\mathbf{Q}) \mathbf{w}(\mathbf{Q})=[K(Q) \mathbf{w}(\mathbf{Q})]^{\mathrm{e}}-\sum_{\mathrm{m}=1}^{\mathrm{N}_{\mathrm{ce}}}\left[\int_{\Omega_{\mathrm{m}}}^{\mathbf{k}_{\sim}^{*}}{ }^{*}(\mathbf{Q}, \mathbf{P}) \underset{\sim}{\Psi^{\mathrm{T}}}(\mathbf{P}) \mathrm{d} \Omega_{\mathrm{m}}(\mathbf{p})\right] \underset{\sim}{\mathbf{M}_{\mathrm{m}}^{0(\mathrm{~N})}}
$$


onde: $[\mathbf{K}(\mathbf{Q}) \mathbf{w}(\mathbf{Q})]^{\text {e }}$ corresponde à equação de $\mathbf{w}(\mathbf{Q})$ do problema sem considerar momentos iniciais, dada por (4.22).

Denominando-se de $\mathbf{e}^{\mathrm{m}}(\mathbf{Q})$ a integral sobre cada célula $\Omega_{\mathrm{m}}$ que aparece na equação (5.64) referente ao deslocamento $\mathbf{w}$ de um ponto do contorno, tem-se:

$$
\underset{\sim}{\mathbf{e}^{\mathrm{m}}}(\mathrm{Q})=-\int_{\Omega_{\mathrm{m}}}\left[\mathbf{w}_{, 11}^{*}(Q, p) \quad 2 \mathbf{w}_{, 12}^{*}(Q, p) \quad \mathbf{w}_{, 22}^{*}(Q, p)\right]{\underset{\sim}{\Psi}}^{\mathrm{T}}(P) \mathrm{d} \Omega_{\mathrm{m}}(\mathbf{p})
$$

Determina-se, portanto, nove coeficientes ao fazer o cálculo de $\mathbf{e}^{\mathbf{m}}(\mathbf{Q})$ sobre uma determinada célula. Genericamente, os mesmos podem ser representados por:

$$
\underset{\mathbf{k l}}{\mathbf{e}^{\mathbf{m}}}(\mathbf{Q})=-\int_{\Omega_{\mathrm{m}}} \mathbf{w}_{, \mathbf{k l}}{ }^{\mathrm{k}}(\mathbf{Q}, \mathbf{P}) \xi_{\alpha}^{\mathbf{p}}(\mathbf{P}) \mathbf{d} \Omega_{\mathbf{m}}(\mathbf{p}) \quad(\mathrm{k}, \mathrm{l}=1,2 ; \alpha=1,2,3)
$$

Substituindo-se nesta última os valores de $\mathbf{w}_{, \mathbf{k l}}^{*}$, dado em (5.16), e $\xi_{\alpha}^{\mathbf{p}}$, dado em (5.59), e considerando-se que $\mathbf{r},_{\mathbf{k}}$ e $\mathbf{r},{ }_{\mathbf{l}}$ são funções apenas do ângulo $\theta$, indicado na figura (5.4), pode-se escrever $\mathbf{d} \Omega_{\mathrm{m}}$ como função de $\mathbf{d r}$ e $\mathbf{d} \theta$ (equação 5.20), chegando-se à integral:

$$
\begin{aligned}
& \mathbf{e}_{\mathrm{kl}}^{\mathrm{m}}(\mathbf{Q})=-\frac{1}{4 \pi \mathrm{D}} \int_{\theta_{1}}^{\theta_{\mathbf{R}_{1}}(\theta)} \int_{\mathbf{R}_{2}(\theta)}\left(\mathbf{r},{ }_{k} \mathbf{r}, \delta_{1}+\delta_{k l} \ln \mathbf{r}\right)\left[\xi_{\alpha}^{\mathrm{q}}+\right. \\
& \left.+\frac{\mathbf{r}}{2 \mathrm{~A}}\left(\mathbf{b}^{\alpha} \cos \theta+\mathbf{a}^{\alpha} \operatorname{sen} \theta\right)\right] \operatorname{rdrd} \theta
\end{aligned}
$$

Fazendo-se a integração em relação a r, tem-se que:

$$
\begin{aligned}
& \mathbf{e}_{\mathrm{kl}}^{\mathbf{m}}(\mathbf{Q})=-\frac{1}{4 \pi \mathrm{D}} \int_{\theta_{1}}^{\theta_{3}} \xi_{\alpha}^{\mathrm{q}}\left\{\frac{\mathbf{R}_{2}^{2}(\theta)}{2}\left[\mathbf{r},{ }_{\mathrm{k}} \mathbf{r},{ }_{1}+\delta_{\mathrm{kl}}\left(\ln \mathbf{R}_{2}(\theta)-\frac{1}{2}\right)\right]+\right. \\
& \left.-\frac{\mathbf{R}_{1}^{2}(\theta)}{2}\left[\mathbf{r},{ }_{k}, r_{1}+\delta_{k l}\left(\ln \mathbf{R}_{1}(\theta)-\frac{1}{2}\right)\right]\right\} \mathbf{d} \theta+
\end{aligned}
$$




$$
\begin{aligned}
& -\frac{1}{4 \pi \mathbf{D}} \int_{\theta_{1}}^{\theta_{3}}\left(\mathbf{b}^{\alpha} \cos \theta+\mathbf{a}^{\alpha} \operatorname{sen} \theta\right)\left\{\frac{\mathbf{R}_{2}^{3}(\theta)}{6 \mathbf{A}}\left[\mathbf{r},{ }_{\mathbf{k}} \mathbf{r},{ }_{1}+\delta_{\mathrm{kl}}\left(\ln \mathbf{R}_{2}(\theta)-\frac{1}{3}\right)\right]+\right. \\
& \left.-\frac{\mathbf{R}_{1}^{3}(\theta)}{\mathbf{6 A}}\left[\mathbf{r},_{\mathbf{k}} \mathbf{r},_{1}+\delta_{\mathrm{kl}}\left(\ln \mathbf{R}_{1}(\theta)-\frac{1}{3}\right)\right]\right\} \mathbf{d} \theta
\end{aligned}
$$

onde: $\mathbf{R}_{\mathbf{1}}(\theta)$ e $\mathbf{R}_{\mathbf{2}}(\theta)$ são dados por (5.61).

Analisando-se os termos da equação (5.68), pode-se concluir que, mesmo quando o ponto de carregamento $\mathbf{q}$ coincidir com um dos vértices do triângulo, nenhuma singularidade ocorre no cálculo das integrais; assim, estas podem ser calculadas numericamente com relação a $\theta$, visto que o valor de $\mathbf{R}_{\mathbf{1}}(\theta)$ torna-se nulo. Para um ponto $\mathbf{q}$ fora da célula, a integração é feita de $\theta_{1}$ à $\theta_{2}$ e então de $\theta_{2}$ à $\theta_{3}$, conforme a figura (5.4).

\subsubsection{Integração sobre as células referente à equação das curvaturas de um ponto interno}

No cálculo das curvaturas de um ponto interno q, que são dadas pela equação (5.36), os coeficientes de $\overline{\mathbf{e}}_{\mathbf{i j k l}}^{*}(\mathbf{q}, \mathbf{p})$, dados por (5.34), são obtidos através de integrações sobre as células, usando-se o mesmo procedimento descrito anteriormente. Pode-se, então, expressar cada coeficiente genérico, proveniente de uma célula $\Omega_{\mathrm{m}}$, como: 


$$
\overline{\mathbf{e}}_{\mathrm{ijkl}}^{-\mathbf{m}}(\mathbf{q})=-\int_{\Omega_{\mathrm{m}}} \mathbf{w}_{, \mathrm{ijk} \mathbf{l}}^{*}(\mathbf{q}, \mathbf{P}) \xi_{\alpha}^{\mathbf{p}}(\mathbf{P}) \mathbf{d} \Omega_{\mathbf{m}}(\mathbf{p}) \quad(\mathrm{i}, \mathrm{j}, \mathrm{k}, 1=1,2 ; \alpha=1,2,3)
$$

Desse modo, em uma determinada célula, são calculados nove coeficientes para cada componente de curvatura $\mathbf{w},{ }_{\mathrm{ij}}$. Os valores de $\mathbf{w}_{, \mathbf{i j k}}{ }^{*}(\mathbf{q}, \mathbf{P})$, podem ser expressos na forma:

$$
\mathbf{w}_{, \mathrm{ijkl}}^{*}(\mathbf{q}, P)=-\frac{1}{4 \pi D r^{2}} \mathbf{f}_{\mathrm{ijkl}}(\theta)
$$

onde: $\mathbf{f}_{\mathrm{ijkl}}(\theta)$ depende apenas do ângulo $\theta$, pois possui apenas as derivadas do raio em relação às direções $\mathbf{x}_{1} \mathrm{e} \mathbf{x}_{2}$.

Substituindo-se (5.70) e (5.59) em (5.69) e fazendo-se a integração sobre $\mathbf{r}$, obtémse:

$$
\begin{gathered}
-\mathbf{e}_{\mathrm{ijkl}}(\mathbf{q})=\frac{1}{4 \pi D} \int_{\theta_{1}}^{\theta_{3}} \mathbf{f}_{\mathrm{ijkl}}(\theta)\left[\xi_{\alpha}^{\mathrm{q}} \ln \mathbf{R}_{2}(\theta)+\left(\mathbf{b}^{\alpha} \cos \theta+\mathbf{a}^{\alpha} \operatorname{sen} \theta\right) \frac{\mathbf{R}_{2}(\theta)}{2 \mathrm{~A}}\right] \mathrm{d} \theta+ \\
-\frac{1}{4 \pi D} \int_{\theta_{1}}^{\theta_{3}} \mathbf{f}_{\mathrm{ijkl}}(\theta)\left[\xi_{\alpha}^{q} \ln \mathbf{R}_{1}(\theta)+\left(\mathbf{b}^{\alpha} \cos \theta+\mathbf{a}^{\alpha} \operatorname{sen} \theta\right) \frac{\mathbf{R}_{1}(\theta)}{2 \mathrm{~A}}\right] \mathbf{d} \theta
\end{gathered}
$$

Essas integrais podem ser feitas numericamente em relação a $\theta$, no caso em que $\mathbf{R}_{\mathbf{1}}(\theta)$ for zero e o ponto $\mathbf{q}$ não coincidir com o vértice $\alpha$ do triângulo, pois nesse caso, $\xi_{\alpha}^{\mathbf{q}}$ é zero. Porém, no caso em que $\mathbf{R}_{\mathbf{1}}(\theta)$ for zero e o ponto $\mathbf{q}$ coincidir com o vértice $\alpha$ do triângulo, tem-se que $\xi_{\alpha}^{\mathbf{q}}=\mathbf{1}$, e, portanto, a segunda integral somente pode ser interpretada no sentido do valor principal de Cauchy. Logo, deve-se reescrever a expressão (5.72), substituindo-se $\mathbf{R}_{\mathbf{1}}(\theta)$ por um pequeno valor constante $\boldsymbol{\varepsilon}$, conforme indicado na figura (5.5), e tomando-se o limite, quando $\varepsilon$ tende a zero, ou seja:

$$
\begin{aligned}
& \mathbf{e}_{\mathrm{ijkl}} \mathbf{m}(\mathbf{q})=\frac{1}{4 \pi D} \int_{\theta_{1}}^{\theta_{2}} \mathbf{f}_{\mathrm{ijkl}}(\theta)\left[\xi_{\alpha}^{q} \ln \mathbf{R}_{2}(\theta)+\left(\mathbf{b}^{\alpha} \cos \theta+\mathbf{a}^{\alpha} \operatorname{sen} \theta\right) \frac{\mathbf{R}_{2}(\theta)}{2 \mathrm{~A}}\right] \mathbf{d} \theta+ \\
& -\frac{1}{4 \pi D} \lim _{\varepsilon \rightarrow 0}\left[\xi_{\alpha}^{q} \ln \varepsilon \int_{\theta_{1}}^{\theta_{2}} \mathbf{f}_{\mathrm{ijkl}}(\theta) \mathrm{d} \theta+\frac{\varepsilon}{2 \mathrm{~A}} \int_{\theta_{1}}^{\theta_{2}} \mathbf{f}_{\mathrm{ijkl}}(\theta)\left(\mathbf{b}^{\alpha} \cos \theta+\mathbf{a}^{\alpha} \operatorname{sen} \theta\right) \mathrm{d} \theta\right]
\end{aligned}
$$

onde $\mathbf{f}_{\mathrm{ijkl}}(\theta)$ tem a seguinte propriedade: 


$$
\int_{0}^{2 \pi} \mathbf{f}_{\mathrm{ijkl}}(\theta) \mathrm{d} \theta=\mathbf{0}
$$

isto é, a soma das integrais de todas as células que concorrem no ponto q é nula, uma vez que, para qualquer célula, tem-se: $\mathbf{r},{ }_{1}=\cos \theta$ e $\mathbf{r}_{2}=\operatorname{sen} \theta$.

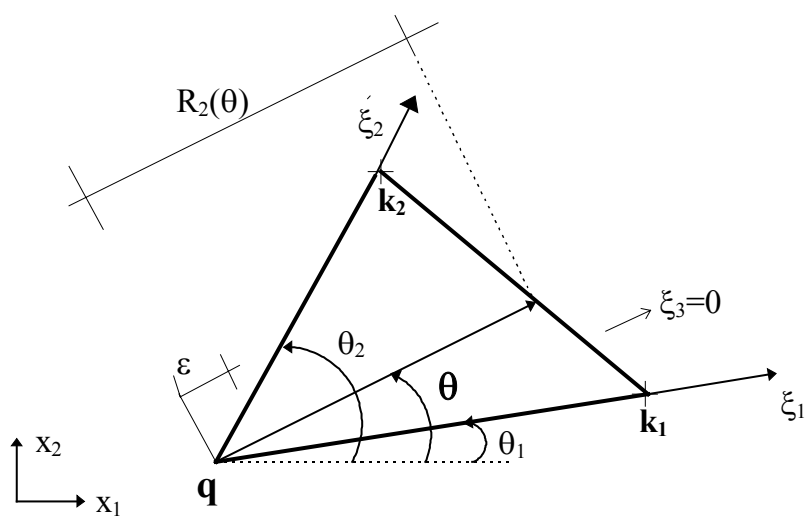

FIGURA 5.5 - Sistema de coordenadas cilíndricas, com origem no vértice da célula

Portanto, a primeira e a última parcela do limite tornam-se nulas tomando-se os limites $\theta_{1}=\mathbf{0}$ e $\theta_{2}=\mathbf{2} \pi$ e considerando-se a equação (5.73). Assim, a equação (5.72) resulta apenas na parcela relativa a $\mathbf{R}_{\mathbf{2}}(\theta)$, que pode ser integrada numericamente em relação a $\theta$, através da fórmula de quadratura de Gauss.

\subsubsection{Integração sobre as células referente à equação das derivadas das curvaturas de um ponto interno}

O mesmo procedimento de integração sobre as células, deve ser usado no cálculo dos coeficientes de $\mathbf{e}_{\beta \mathbf{k} \mathbf{l}}^{*}(\mathbf{q}, \mathbf{p})$, dados por (5.48), que aparecem na equação(5.49) das derivadas das curvaturas de um ponto interno q. Genericamente, cada coeficiente proveniente de uma célula $\Omega_{\mathrm{m}}$, pode ser escrito como:

$$
\mathbf{e}_{\beta \mathbf{k l}}^{\mathbf{m}}(\mathbf{q})=-\int_{\Omega_{\mathrm{m}}} \frac{\partial \mathbf{w}_{, \mathrm{mmkl}}^{*}}{\partial \mathbf{x}_{\beta}(\mathbf{q})}(\mathbf{q}, \mathbf{P}) \xi_{\alpha}^{\mathbf{p}}(\mathbf{P}) \mathbf{d} \Omega_{\mathbf{m}}(\mathbf{p}), \quad(\beta, \mathrm{k}, 1=1,2 ; \alpha=1,2,3)
$$

As funções a serem integradas, nesta equação, podem ser expressas como: 


$$
\frac{\partial \mathbf{w}_{, \text {mmkl }}^{*}}{\partial \mathbf{x}_{\beta}(\mathbf{q})}(\mathbf{q}, \mathbf{P})=-\frac{1}{4 \pi D r^{3}} \mathbf{f}_{\beta \mathbf{k l}}(\theta)
$$

onde: $\mathbf{f}_{\beta \mathbf{k l}}(\theta)$ são funções das derivadas de $\mathbf{r}$ em relação às direções $\mathbf{x}_{\mathbf{1}}$ e $\mathbf{x}_{\mathbf{2}} \mathrm{e}$, portanto, dependem apenas do ângulo $\theta$.

Substituindo-se (5.75) e (5.59) em (5.74) e fazendo-se a integração sobre $\mathbf{r}$, obtémse:

$$
\begin{aligned}
\mathbf{e}_{\mathrm{ijkl}}^{\mathbf{m}}(\mathbf{q}) & =\frac{1}{4 \pi} \int_{\theta_{1}}^{\theta_{3}} \mathbf{f}_{\beta \mathbf{k l}}(\theta)-\left[\frac{\xi_{\alpha}^{q}}{\mathbf{R}_{2}(\theta)}+\left(\mathbf{b}^{\alpha} \cos \theta+\mathbf{a}^{\alpha} \operatorname{sen} \theta\right) \frac{\ln \mathbf{R}_{2}(\theta)}{2 \mathrm{~A}}\right] \mathbf{d} \theta+ \\
& -\frac{1}{4 \pi} \int_{\theta_{1}}^{\theta_{3}} \mathbf{f}_{\beta \mathbf{k l}}(\theta)\left[-\frac{\xi_{\alpha}^{q}}{\mathbf{R}_{1}(\theta)}+\left(\mathbf{b}^{\alpha} \cos \theta+\mathbf{a}^{\alpha} \operatorname{sen} \theta\right) \frac{\ln \mathbf{R}_{1}(\theta)}{2 \mathrm{~A}}\right] \mathbf{d} \theta
\end{aligned}
$$

Estas integrais podem ser feitas numericamente em relação a $\theta$, a não ser quando $\mathbf{R}_{\mathbf{1}}(\theta)$ for nulo. Assim, nesse caso, deve-se verificar as duas parcelas da segunda integral de (5.76), envolvendo $\mathbf{R}_{\mathbf{1}}(\theta)$. A primeira parcela envolve a integral:

$$
\int_{\theta_{1}}^{\theta_{3}} \mathbf{f}_{\beta \mathbf{k l}}(\theta)\left[-\frac{\xi_{\alpha}^{q}}{\mathbf{R}_{1}(\theta)}\right] \mathbf{d} \theta
$$

que apresenta singularidade, quando o ponto $\mathbf{q}$ coincidir com o vértice $\alpha$ do triângulo e, portanto, $\xi_{\alpha}^{\mathbf{q}}=\mathbf{1}$. Considerando-se, porém, a propriedade da função $\mathbf{f}_{\beta \mathbf{k l}}(\theta)$, dada por (5.73), a parcela (5.77) é nula, pois:

$$
\int_{\theta_{1}}^{\theta_{3}} \mathbf{f}_{\beta \mathbf{k l}}(\theta) \mathrm{d} \theta=\mathbf{0}
$$

A segunda parcela de (5.76), envolvendo $\mathbf{R}_{\mathbf{1}}(\theta)$, que é dada por:

$$
\int_{\theta_{1}}^{\theta_{3}} \mathbf{f}_{\beta \mathbf{k}}(\theta)\left[\left(\mathbf{b}^{\alpha} \cos \theta+\mathbf{a}^{\alpha} \operatorname{sen} \theta\right) \frac{\ln \mathbf{R}_{1}(\theta)}{2 A}\right] \mathbf{d} \theta
$$


apresenta singularidade quando o ponto $\mathbf{q}$ coincidir com qualquer vértice da célula, pois, neste caso, $\mathbf{R}_{\mathbf{1}}(\theta)$ é nulo. Entretanto, quando $\mathbf{R}_{\mathbf{1}}(\theta)$ for substituído pelo valor $\varepsilon$ (figura 5.5), fazendo-se o limite para $\varepsilon$ tendendo a zero, a equação (5.79) se torna:

$$
\lim _{\varepsilon \rightarrow 0} \frac{\ln \varepsilon}{2 A} \int_{\theta_{1}}^{\theta_{2}} \mathbf{f}_{\beta \mathbf{k}}(\theta)\left(\mathbf{b}^{\alpha} \cos \theta+\mathbf{a}^{\alpha} \operatorname{sen} \theta\right) \mathbf{d} \theta
$$

Considerando-se que $\mathbf{r},{ }_{1}=\cos \theta, \mathbf{r}_{2}=\operatorname{sen} \theta$, a simetria em torno do ponto $\mathbf{q}$ e também a característica da função $\mathbf{f}_{\beta \mathbf{k l}}(\theta)$, para a qual se tem:

$$
\int_{0}^{2 \pi} \mathbf{r},{ }_{1} \mathbf{f}_{\beta \mathbf{k l}}(\theta) \mathrm{d} \theta=\mathbf{0}
$$

e

$$
\int_{0}^{2 \pi} \mathbf{r}, \mathbf{f}_{\beta \mathbf{k l}}(\theta) \mathrm{d} \theta=\mathbf{0}
$$

na equação (5.76), permanecem apenas as integrais relativas a $\mathbf{R}_{\mathbf{2}}(\theta)$, as quais podem ser feitas numericamente.

\subsection{Cálculo dos deslocamento es esforços no contorno e no domínio}

\subsubsection{Sistema de equações}

Após a soma dos coeficientes de $\mathbf{h}^{\mathbf{j}}(\mathbf{Q})$ e $\mathbf{g}^{\mathbf{j}}(\mathbf{Q})$ de todos os elementos do contorno e da soma de $\mathbf{e}^{\mathrm{m}}(\mathbf{Q})$ para todas as células do domínio, agrupando-se os coeficientes multiplicativos de um mesmo valor nodal, e escrevendo o deslocamento $\mathbf{w}(\mathbf{Q})$ em função dos deslocamentos do elemento ao qual pertence, como é mostrado no item (4.2.3), pode-se escrever a equação (5.64), referente ao deslocamento de um ponto do contorno, em sua forma matricial da seguinte maneira:

$$
\underset{\sim}{\mathbf{H}}(\mathbf{Q}) \underset{\sim}{\mathbf{U}}+\mathbf{H}_{\mathbf{c}}(\mathbf{Q}) \mathbf{w}_{\mathbf{c}}=\underset{\sim}{\mathbf{G}(Q)} \underset{\sim}{\mathbf{P}}+\mathbf{G}_{\mathbf{c}}(\mathbf{Q}) \mathbf{R}_{\mathbf{c}}+\mathbf{T}(\mathbf{Q})+\underset{\sim}{\mathbf{E}}(\mathbf{Q}) \underset{\sim}{\mathbf{M}^{\mathbf{0}}}
$$

onde: 
- $\underset{\sim}{\mathbf{H}}(\mathbf{Q}), \underset{\sim}{\mathbf{G}}(\mathbf{Q}), \mathbf{H}_{\mathbf{c}}(\mathbf{Q}), \mathbf{G}_{\mathbf{c}}(\mathbf{Q}) \underset{\sim}{\mathbf{U}}, \underset{\sim}{\mathbf{P}}, \mathbf{w}_{\mathbf{c}}$, e $\mathbf{R}_{\mathbf{c}}$ são os vetores indicados em (4.25),

- $\mathbf{T}(\mathbf{Q})$ é o coeficiente dado em (4.25),

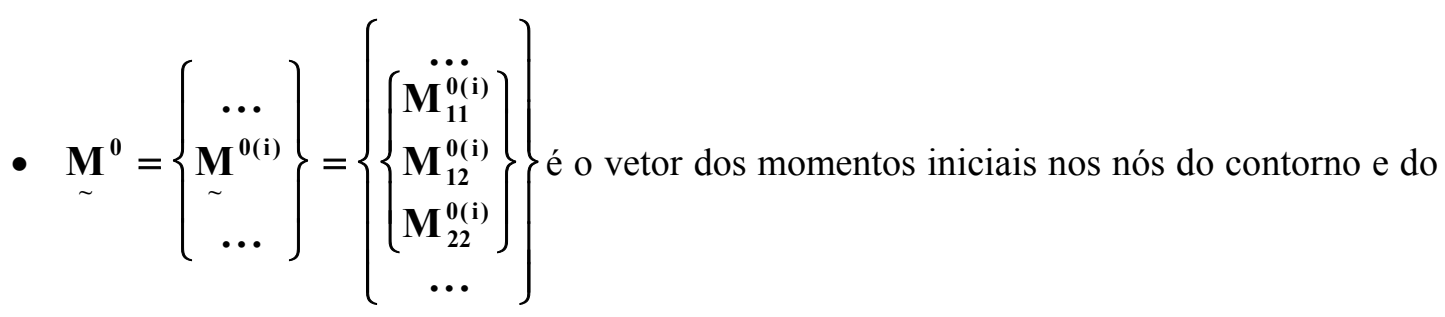
domínio e tem dimensão $3\left(\mathrm{~N}_{\mathrm{t}}\right)$ x 1 , onde $\mathbf{N}_{\mathbf{t}}$ é o número de pontos do contorno $\mathbf{N}_{\mathbf{n}}$ mais o número de pontos internos $\mathbf{N}_{\mathbf{i}}$,

- $\mathbf{E}(\mathbf{Q})$ representa a infuência do campo de momentos iniciais sobre o valor do deslocamento $\mathbf{w}(\mathbf{Q})$ do ponto do contorno. Seus coeficientes são obtidos por (5.68).

Como foi visto no item (4.3), o sistema de equações pode ser obtido de duas maneiras diferentes. Assim, escrevendo-se duas equações do deslocamento transversal w correspondentes a cada nó do contorno e uma para cada canto, obtém-se o sistema de equações:

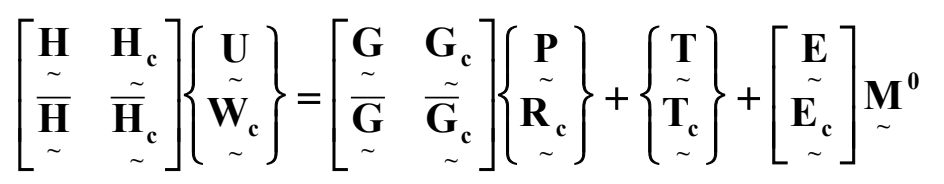

onde: $\quad \mathbf{M}^{0}$ é o vetor dos momentos iniciais, de dimensão $3 \mathrm{~N}_{\mathrm{t}} \mathrm{x}$ 1,

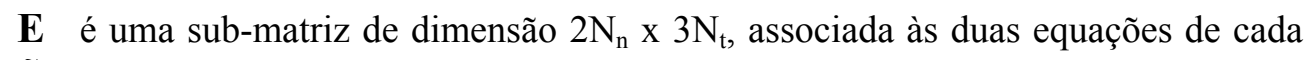
nó do contorno,

$\mathbf{E}_{\mathbf{c}}$ é uma sub-matriz de dimensão $\mathrm{N}_{\mathrm{c}} \times 3 \mathrm{~N}_{\mathrm{t}}$, associada às equações dos cantos,

todos os outros vetores e sub-matrizes estão definidos em (4.36).

Com a resolução do sistema (5.84) obtém-se as incógnitas do contorno.

\subsubsection{Deslocamentos e esforços no domínio}


Analogamente, ao item (5.4.1), os deslocamentos $\mathbf{w}(\mathbf{q})$ de $\mathbf{N}_{\mathbf{i}}$ pontos internos são dados pela equação matricial:

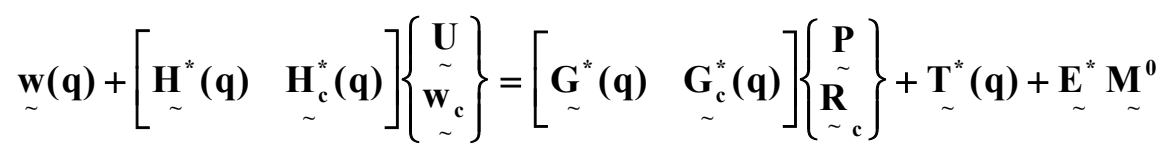

onde: $\quad \mathbf{E}^{*}$ é uma matriz de dimensão $\mathrm{N}_{\mathrm{i}}$ x $3 \mathrm{~N}_{\mathrm{t}}$, cujos coeficientes são obtidos de (5.68), e os outros vetores e matrizes estão definidos em (4.47).

De forma análoga, obtém-se a equação matricial das curvaturas $\mathbf{w},_{\mathrm{ij}}$ em $\mathbf{N}_{\mathbf{i}}$ pontos internos, que é dada por:

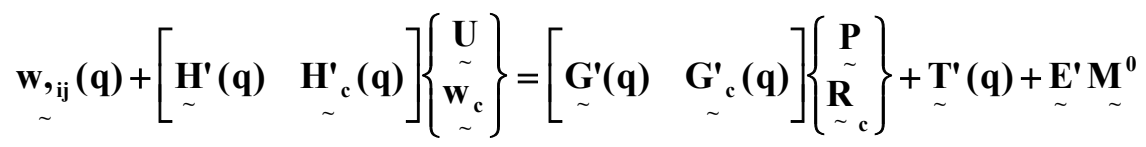

onde:

- $\mathbf{E}^{\prime}$ é uma matriz de dimensão $3 \mathrm{~N}_{\mathrm{i}} \times 3 \mathrm{~N}_{\mathrm{t}}$, que representa a influência dos momentos iniciais nas curvaturas dos pontos internos e cujos coeficientes são obtidos somando-se os termos $\overline{\mathbf{g}}_{\mathrm{ijkl}}(\mathbf{q})$, dados por (5.35), aos coeficientes de $\overline{\mathbf{e}}_{\mathrm{ijk \mathbf {l }}}^{\mathbf{m}}(\mathbf{q}, \mathbf{p})$, dados por (5.71), que multiplicam as componentes $\mathbf{M}_{\mathbf{k l}}^{\mathbf{0}}$ do ponto $\mathbf{q}$.

- os outros vetores e matrizes estão definidos em (4.57).

De forma análoga ao que foi mostrado no item (4.5), pode-se escrever a equação dos momentos elásticos, dada por (5.6), em $\mathbf{N}_{\mathbf{i}}$ pontos internos, da seguinte maneira:

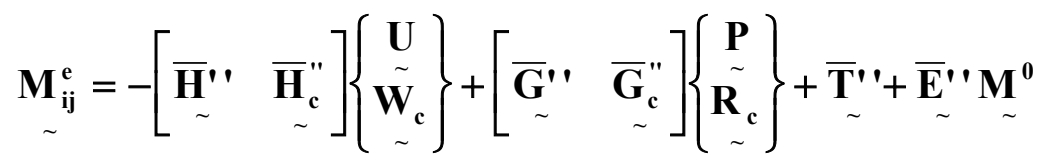

onde:

- $\overline{\mathbf{E}}^{\prime}$ ' tem dimensão $3 \mathrm{~N}_{\mathrm{i}}$ x $3 \mathrm{~N}_{\mathrm{n}}$, e seus coeficientes são obtidos a partir dos coeficientes de

$\mathbf{E}^{\prime}$ da equação (5.86), de maneira análoga ao que foi feito para $\overline{\mathbf{H}}^{\prime}$ ', no item (4.5),

- os outros vetores e matrizes estão definidos na equação (4.58). 
Os esforços cortantes nos pontos internos são dados pela equação (5.8), onde os valores de $\mathbf{w},_{\mathbf{k} k \beta}$ são calculadas pela equação (5.49). Assim, procedendo-se da mesma maneira ao que foi feito para a obtenção da equação das curvaturas nos pontos internos, obtém-se a equação da derivada das curvaturas $\mathbf{w},{ }_{\mathbf{k k} \beta}$ para $\mathbf{N}_{\mathbf{i}}$ pontos internos:

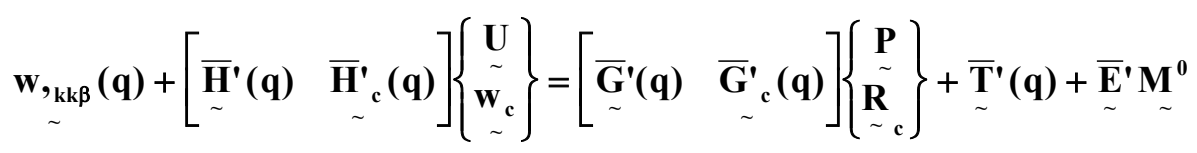

onde:

- $\overline{\mathbf{E}}^{\prime}$ é uma matriz de dimensão $2 \mathrm{~N}_{\mathrm{i}} \times 3 \mathrm{~N}_{\mathrm{t}}$, que representa a influência dos momentos iniciais nos valores das derivadas das curvaturas dos pontos internos e cujos coeficientes são obtidos somando-se os termos $\overline{\mathbf{q}}_{\beta}^{0}(\mathbf{q})$, dados por (5.47), aos coeficientes de $\mathbf{e}_{\beta \mathbf{k}}^{\mathbf{m}}(\mathbf{q}, \mathbf{p})$, dados por (5.76), referentes às componentes $\mathbf{M}_{11}^{\mathbf{0}}$ e $\mathbf{M}_{22}^{\mathbf{0}}$ do ponto $\mathbf{q}$.

- os outros vetores e matrizes estão definidos em (4.69).

\subsubsection{Momentos $\left(\mathrm{M}_{\mathrm{ij}}\right)$ nos pontos do contorno}

Os momentos $\overline{\mathbf{M}}_{\mathrm{ij}}$ nos pontos do contorno, em relação ao sistema local de coordenadas (figura 4.10), de acordo com a equação (5.5), são dados por:

$$
\overline{\mathbf{M}}_{\mathrm{ij}}=\overline{\mathbf{M}}_{\mathrm{ij}}^{\mathrm{e}}-\overline{\mathbf{M}}_{\mathrm{ij}}^{\mathbf{0}}
$$

Como foi visto no item (4.6), tem-se que $\overline{\mathbf{M}}_{11}=\mathbf{M}_{\mathbf{n}}$, valor conhecido após a resolução do sistema (5.84). Logo, obtêm-se:

$$
\begin{aligned}
& \overline{\mathbf{M}}_{11}=\mathbf{M}_{\mathrm{n}} \\
& \overline{\mathbf{M}}_{12}=\overline{\mathbf{M}}_{12}^{\mathrm{e}}-\overline{\mathbf{M}}_{12}^{0} \\
& \overline{\mathbf{M}}_{22}=\overline{\mathbf{M}}_{22}^{\mathrm{e}}-\overline{\mathbf{M}}_{22}^{0}
\end{aligned}
$$

Substituindo-se as equações (5.90) em (5.89), obtêm-se os momentos elásticos $\overline{\mathbf{M}}_{\mathrm{ij}}^{\mathrm{e}}$ nos pontos do contorno, os quais são dados por: 


$$
\overline{\mathbf{M}}_{11}^{\mathrm{e}}=\mathbf{M}_{\mathrm{n}}+\overline{\mathbf{M}}_{11}^{0}
$$

e as componentes $\overline{\mathbf{M}}_{12}^{\mathrm{e}}$ e $\overline{\mathbf{M}}_{22}^{\mathrm{e}}$ são dadas respectivamente por (4.74) e (4.80).

Considerando-se as equações de transformação de coordenadas, dadas por (4.81) e (4.82), obtém-se o valor de $\overline{\mathbf{M}}_{11}^{0}$ em função dos momentos iniciais no sistema global de coordenadas $\left(\mathrm{X}_{1}, \mathrm{X}_{2}\right)$ :

$$
\overline{\mathbf{M}}_{11}^{0}=\left\{\begin{array}{lll}
\operatorname{sen}^{2} \alpha & -2 \operatorname{sen} \alpha \cos \alpha & \cos ^{2} \alpha
\end{array}\right\}\left\{\begin{array}{l}
\mathbf{M}_{11}^{0} \\
\mathbf{M}_{12}^{0} \\
\mathbf{M}_{22}^{0}
\end{array}\right\}
$$

Assim, de maneira análoga ao mostrado no item (4.6), obtêm-se os momentos elásticos de um ponto do contorno $\mathbf{Q}$, em função dos deslocamentos, esforços e momentos iniciais dos nós do elemento $\mathbf{j}$ ao qual pertence:

$$
\left\{\mathbf{M}^{\mathrm{e}}\right\}_{\mathrm{Q}}=[\mathbf{h}]_{\mathrm{j}}\{\mathbf{U}\}_{\mathrm{j}}+[\mathbf{g}]_{\mathrm{j}}\{\mathbf{P}\}_{\mathrm{j}}+\left[\mathbf{e}^{\mathrm{\prime}}\right]_{\mathrm{j}}\left\{\mathbf{M}^{0}\right\}_{\mathrm{j}}
$$

onde:

- as matrizes $[\mathrm{h}]$ e $[\mathrm{g}]$ e os vetores $\{\mathrm{U}\}$ e $\{\mathrm{P}\}$, estão indicados em (4.84),

- o vetor $\left\{\mathrm{M}^{0}\right\}$, de dimensão 9 x 1, é composto pelos valores nodais dos momentos iniciais do elemento $\mathbf{j}$,

- a matriz [e"] tem dimensão 3 x 9 e os coeficientes correspondentes a um determinado nó $\mathbf{k}$ do elemento são dados por:

$$
\left[\mathrm{e}^{\prime \prime}\right]_{\mathrm{k}}=\left\{\begin{array}{l}
\operatorname{sen}^{2} \alpha+v \cos ^{2} \alpha \\
\operatorname{sen} \alpha \cos \alpha(v-1) \\
\cos ^{2} \alpha+v \operatorname{sen}^{2} \alpha
\end{array}\right\}\left\{\operatorname{sen}^{2} \alpha-2 \operatorname{sen} \alpha \cos \alpha \cos ^{2} \alpha\right\}
$$

Após acoplar todos os elementos que compõe o contorno, chega-se à equação matricial de momentos para $\mathbf{N}_{\mathbf{n}}$ pontos do contorno, que é dada por:

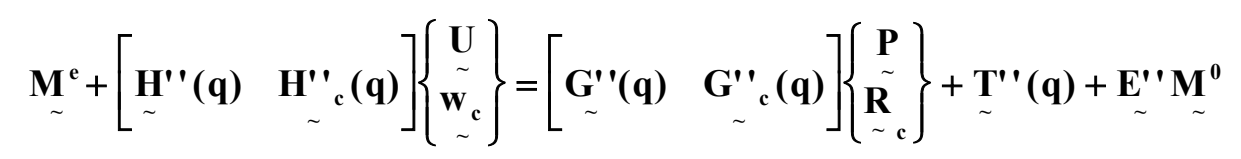

onde: 
- os coeficientes de $\mathbf{E}^{\prime \prime}$, de dimensão $3 \mathrm{~N}_{\mathrm{n}}$ x $3 \mathrm{~N}_{\mathrm{n}}$, estão indicados em (5.94),

- o vetor $\mathbf{M}^{0}$ contém os valores dos momentos iniciais em todos os nós do contorno e tem dimensão $3 \mathrm{~N}_{\mathrm{n}} \times 1$.

- os outros vetores e matrizes estão indicados em (4.85).

Se o nó $\mathbf{Q}$ for um nó de extremidade, e não for duplo, calcula-se os coeficientes referentes aos dois elementos ao qual pertence e faz-se uma média entre os mesmos.

\subsection{Técnica de Solução}

\subsubsection{Cálculo das incógnitas do contorno e deslocamentos w do domínio}

O sistema de equação (5.84), do qual se obtêm as incógnitas de $\mathbf{N}_{\mathbf{n}}$ nós do contorno e $\mathbf{N}_{\mathbf{c}}$ cantos, e a equação matricial (5.85), da qual obtêm-se os deslocamentos $\mathbf{w}$ em $\mathbf{N}_{\mathbf{i}}$ pontos internos, são montadas em uma mesma equação matricial, porém resolve-se primeiro o sistema de equações, para depois, com os valores das variáveis no contorno, se obter os deslocamentos nos pontos internos. Assim, escrevendo-se as equações (5.84) e (5.85) em uma mesma equação matricial, tem-se:

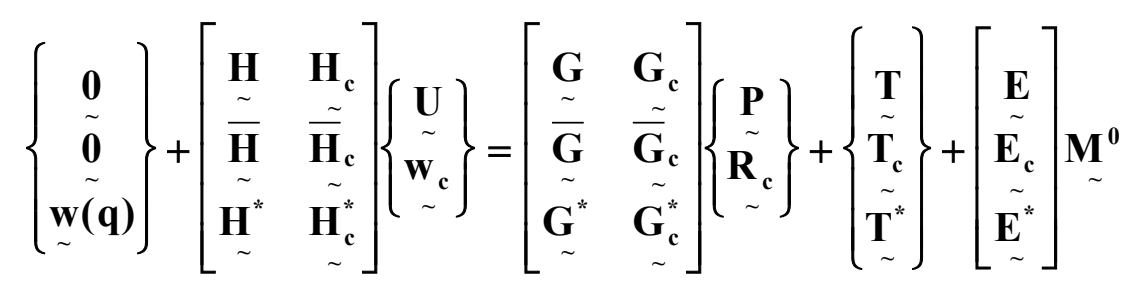

onde 0 são vetores nulos, referentes às duas equações de cada ponto do contorno e às equações dos cantos.

De uma forma simplificada, a equação (5.96) pode ser escrita da seguinte forma:

$$
\underset{\sim}{\mathbf{U}}(\mathbf{q})+\underset{\sim}{\overline{\overline{\mathbf{H}}}} \underset{\sim}{\mathbf{U}}=\underset{\sim}{\overline{\overline{\mathbf{G}}}} \underset{\sim}{\overline{\mathbf{P}}}+\underset{\sim}{\overline{\mathbf{T}}}+\underset{\sim}{\overline{\mathbf{E}}} \underset{\sim}{\mathbf{M}^{\mathbf{0}}}
$$

onde:

- o vetor de deslocamentos $\overline{\mathbf{U}}(\mathbf{q})$ de dimensão $\left(2 \mathrm{~N}_{\mathrm{n}}+\mathrm{N}_{\mathrm{c}}+\mathrm{N}_{\mathrm{i}}\right)$ x 1 é nulo para as linhas referentes ao sistema de equações, 
- as matrizes $\underset{\sim}{\overline{\mathbf{H}}}$ e $\underset{\sim}{\overline{\mathbf{G}}}$ têm dimensão $\left(2 \mathrm{~N}_{\mathrm{n}}+\mathrm{N}_{\mathrm{c}}+\mathrm{N}_{\mathrm{i}}\right) \mathrm{x}\left(2 \mathrm{~N}_{\mathrm{n}}+\mathrm{N}_{\mathrm{c}}\right)$ e a matriz $\underset{\sim}{\overline{\mathbf{E}}}$ dimensão $\left(2 \mathrm{~N}_{\mathrm{n}}+\mathrm{N}_{\mathrm{c}}+\mathrm{N}_{\mathrm{i}}\right) \times 3\left(\mathrm{~N}_{\mathrm{n}}+\mathrm{N}_{\mathrm{i}}\right)$,

- os vetores $\bar{\sim} \overline{\mathbf{U}}$ e $\underset{\sim}{\mathbf{P}}$, de dimensão $\left(2 \mathrm{~N}_{\mathrm{n}}+\mathrm{N}_{\mathrm{c}}\right)$ x 1, contém os deslocamentos e esforços do contorno,

- o vetor $\underset{\sim}{\overline{\mathbf{T}}}$ tem dimensão $\left(2 \mathrm{~N}_{\mathrm{n}}+\mathrm{N}_{\mathrm{c}}+\mathrm{N}_{\mathrm{i}}\right) \times 1$

- o vetor $\mathbf{M}^{0}$, de dimensão $3\left(\mathrm{~N}_{\mathrm{n}}+\mathrm{N}_{\mathrm{i}}\right) \times 1$, contém os momentos iniciais nos pontos do contorno e internos,

O sistema de equações (5.84) pode ser escrito, de forma simplificada, da seguinte maneira:

$$
\bar{\sim}^{*} \underset{\sim}{\mathbf{U}}=\bar{\sim}_{\sim}^{*} \underset{\sim}{\mathbf{P}}+\bar{\sim}^{\overline{\mathbf{T}}^{*}}+\underset{\sim}{\overline{\mathbf{E}}^{*}}{\underset{\sim}{\mathbf{M}}}^{0}
$$

Com a solução do sistema (5.98), obtêm-se as incógnitas do contorno. Então, com esses valores obtêm-se os deslocamentos $\mathbf{w}$ dos pontos internos, resolvendo-se a parte restante da equação (5.96).

Para se obter um procedimento numérico mais conveniente para a análise não linear, são feitas operações matriciais sobre a equação (5.98), visando-se deixar os termos referentes aos momentos iniciais isolados. Assim, armazenam-se todas as incógnitas do contorno num vetor $\underset{\sim}{\mathbf{X}}$, trocando-se as respectivas colunas entre as matrizes $\underset{\sim}{\overline{\mathbf{H}}^{*}}$ e $\overline{\mathbf{G}}^{*}$. Então, somam-se em B os efeitos dos deslocamentos ou esforços prescritos no contorno, ao efeito do carregamento que atua na placa, obtendo-se:

$$
\underset{\sim}{\mathbf{A}} \underset{\sim}{\mathbf{X}}=\underset{\sim}{\mathbf{B}}+\underset{\sim}{\overline{\mathbf{E}}^{*}}{\underset{\sim}{\mathbf{0}}}^{\mathbf{0}}
$$

Multiplicando-se os dois lados dessa equação por $\mathbf{A}^{\mathbf{1}}$, obtêm-se as incógnitas do contorno:

$$
\underset{\sim}{\mathbf{X}}=\underset{\sim}{\mathbf{L}}+\underset{\sim}{\mathbf{R}} \mathbf{M}^{\mathbf{0}}
$$

onde: $\quad \underset{\sim}{\mathbf{L}}=\underset{\sim}{\mathbf{A}^{-1}} \underset{\sim}{\mathbf{B}}$ 


$$
\underset{\sim}{\mathbf{R}}=\underset{\sim}{\mathbf{A}^{-1}} \overline{\mathbf{E}}^{*}
$$

$\mathbf{R}$ representa a influência dos momentos iniciais nos deslocamentos do contorno,

L representa a resposta elástica, sem considerar os momentos iniciais.

Para os deslocamentos internos, de forma análoga ao que foi feito anteriormente, tem-se:

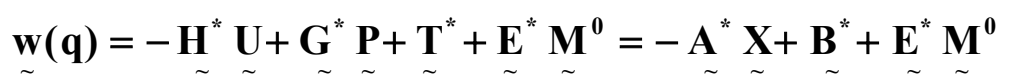

Considerando-se a equação (5.100), obtém-se:

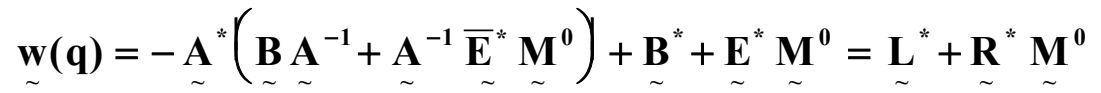

onde: $\quad{\underset{\sim}{R}}^{*}=-\underset{\sim}{\mathbf{A}^{*}}{\underset{\sim}{\mathbf{A}}}^{-1} \bar{\sim}^{*}+\underset{\sim}{\mathbf{E}^{*}}$

$$
\mathbf{L}^{*}=-\underset{\sim}{\mathbf{A}^{*}} \underset{\sim}{\mathbf{B}} \mathbf{A}^{-1}+{\underset{\sim}{\mathbf{B}^{*}}}^{*}
$$

\subsubsection{Cálculo dos esforços nos pontos internos e no contorno}

Pode-se escrever a equação dos momentos elásticos do contorno (5.95) e a equação dos momentos elásticos nos pontos internos (5.87) em uma mesma equação matricial, dada por:

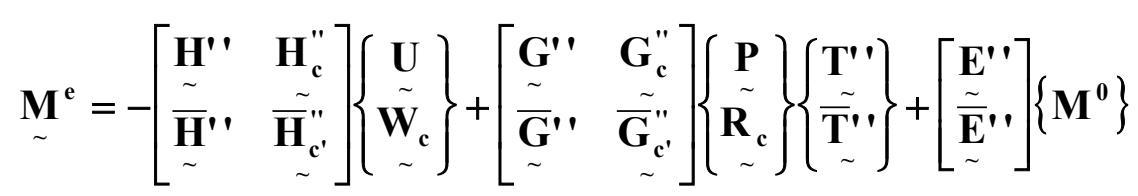

Ou de uma forma simplificada:

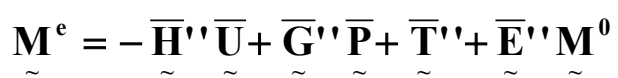

onde: $\quad \overline{\mathbf{H}}^{\prime \prime}$ e $\overline{\mathbf{G}}^{\prime \prime}$, têm dimensão $3\left(\mathrm{~N}_{\mathrm{n}}+\mathrm{N}_{\mathrm{i}}\right) \mathrm{x}\left(2 \mathrm{~N}_{\mathrm{n}}+\mathrm{N}_{\mathrm{c}}\right)$,

$\overline{\mathbf{E}}^{\prime}$ ' tem dimensão $3\left(\mathrm{~N}_{\mathrm{i}}+\mathrm{N}_{\mathrm{n}}\right) \times 3\left(\mathrm{~N}_{\mathrm{n}}+\mathrm{N}_{\mathrm{i}}\right)$, 
$\overline{\mathbf{T}}^{\prime \prime}$ tem dimensão $3\left(\mathrm{~N}_{\mathrm{i}}+\mathrm{N}_{\mathrm{n}}\right) \times 1$.

De forma análoga ao que foi feito para a equação (5.98) e considerando-se a equação (5.100) obtém-se:

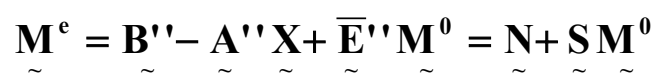

onde: $\quad \underset{\sim}{\mathbf{N}}=\mathbf{B}_{\sim}^{\prime \prime}-\mathbf{A}_{\sim}^{\prime \prime}{ }_{\sim}^{\mathbf{L}}$

$$
\mathbf{S}=\mathbf{E}^{\prime \prime}-\mathbf{A}^{\prime \prime} \mathbf{R}
$$

$\mathbf{S}$ representa a influência dos momentos iniciais nos momentos dos pontos internos e do contorno,

$\mathbf{N}$ representa a resposta elástica, sem considerar os momentos iniciais.

As derivadas das curvaturas, nos pontos internos, são dadas pela equação (5.88), que pode ser escrita na forma:

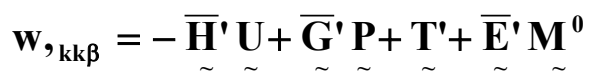

Procedendo-se da mesma maneira que foi feito para a equação dos momentos, temse:

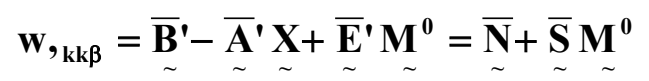

onde: $\overline{\mathbf{N}}=\overline{\mathbf{B}}^{\prime}-\overline{\mathbf{A}}^{\prime} \mathbf{L}$

$$
\bar{\sim} \bar{\sim}=\tilde{\mathbf{E}}^{\prime}-\bar{\sim}_{\sim}^{\mathbf{A}^{\prime}} \underset{\sim}{\mathbf{R}}
$$

Os esforços cortantes são obtidos, substituindo-se os valores de $\mathbf{w},_{\mathbf{k k} \beta}$, dados por (5.113), na equação abaixo:

$$
\mathbf{Q}_{\mathbf{j}}=-\mathbf{D w},_{\mathbf{k k j}}-\mathbf{M}_{\mathrm{ij}, \mathrm{i}}^{\mathbf{i}}
$$


Os momentos são dados por:

$$
\mathbf{M}_{\mathrm{ij}}=\mathbf{M}_{\mathrm{ij}}^{\mathrm{e}}-\mathbf{M}_{\mathrm{ij}}^{\mathbf{0}}
$$

onde $\mathbf{M}_{\mathrm{ij}}^{\mathrm{e}}$ são dados pela equação (5.109).

Como já foi dito, no início do capítulo, a solução não-linear de placas sujeitas à cargas transversais é obtida através da formulação apresentada nesse capítulo, onde admitese a existência de um campo de momentos iniciais na placa. 


\section{MODELOS CONSTITUTIVOS UTILIZADOS}

\subsection{Introdução}

Para o concreto serão considerados dois modelos constitutivos bidimensionais: um modelo elasto-plástico com encruamento isótropo negativo, sendo que o critério de plastificação utilizado é o de Von Mises, onde considera-se que o concreto tem resistência somente à compressão e o modelo de dano de MAZARS (1984). Para a armadura será considerado um modelo elasto-plástico bilinear e uniaxial com encruamento isótropo positivo.

\subsection{Modelo Elasto-Plástico}

\subsubsection{Introdução}

O comportamento elasto-plástico, é caracterizado pelo aparecimento de deformações plásticas na estrutura. Tais deformações são irreversíveis e aparecem quando anula-se a solicitação à que o corpo estava sujeito, se essa última tiver provocado tensões que excederam o limite elástico do material. Um tratamento mais detalhado sobre a teoria da plasticidade pode ser encontrado mas publicações de HILL (1950) e MENDELSON (1968), entre outras.

A teoria da plasticidade é ideal para materiais dúcteis, como o aço, mas é usada para materiais frágeis, como é o caso do concreto, de maneira aproximada. Para materiais frágeis, a ruptura, que é devido à abertura de fissuras, ocorre sem plastificação significativa, pois têm pequena capacidade de deformação, ao contrário dos materiais dúcteis. 
Para modelar o comportamento elasto-plástico, deve-se ter relações explícitas entre tensão e deformação, para a fase elástica e a fase plástica, após o escoamento. Deve-se também ter uma lei de evolução do tensor de deformações plásticas, um critério de escoamento, ou de plastificação, que indica quando haverá escoamento; e no caso de modelos que considerem o encruamento, é necessário uma regra de endurecimento, que governa a variação da tensão de escoamento, em função das deformações plásticas.

\subsubsection{Modelo Elasto-Plástico Perfeito}

No caso uniaxial, o critério de plastificação $\mathbf{f}(\sigma)$ é dado por :

$$
\mathbf{f}(\sigma)=|\sigma|-\sigma_{\mathbf{y}} \leq \mathbf{0}
$$

onde $\sigma$ é a tensão à qual o ponto está submetido e $\sigma_{y}$ é a tensão de plastificação ou de escoamento, considerada constante.

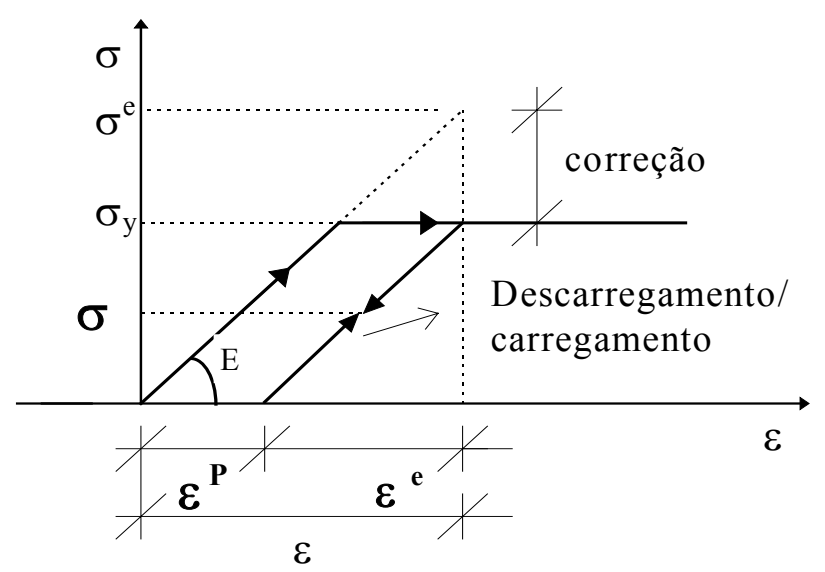

FIGURA 6.1 - Comportamento Elasto-plástico Perfeito

onde $\sigma^{\mathrm{e}}$ é tensão suposta totalmente elástica.

A resposta elasto-plástica fica evidenciada no caminho de descarregamento de um ciclo de tensão, como é mostrado na figura (6.1), onde para $\sigma<\sigma_{\mathbf{y}}$ têm-se um comportamento puramente elástico. Não se admite tensões maiores que $\sigma_{y}$, ou seja, $\mathbf{f}(\sigma)>\mathbf{0}$. Quando isso ocorre em um determinado ponto, deve-se fazer a correção da tensão. Assim, o mesmo sofre descarregamento, pois não é capaz de suportar o estado de 
tensão à que está submetido, havendo redistribuição de tensão para os pontos vizinhos e evolução da deformação plástica no ponto considerado. Se houver um novo carregamento, esse será efetuado sobre a mesma reta do último descarregamento.

Assim sendo, a deformação total $\varepsilon$, no regime plástico, é composta por uma parcela elástica $\varepsilon^{\mathrm{e}}$, e outra plástica $\varepsilon^{\mathrm{p}}$ :

$$
\varepsilon=\varepsilon^{\mathrm{e}}+\varepsilon^{\mathrm{p}}
$$

A tensão é dada por :

$$
\sigma=\mathbf{E} \varepsilon^{\mathbf{e}}=\mathbf{E}\left(\varepsilon-\varepsilon^{\mathbf{p}}\right)
$$

Admitindo-se que $\varepsilon, \sigma$ e $\varepsilon^{\mathbf{p}}$ são funções de tempo, define-se, em particular, $\dot{\boldsymbol{\varepsilon}}^{\mathbf{p}}=\frac{\mathbf{d} \varepsilon^{\mathbf{p}}}{\mathbf{d t}}$ e diz-se que as deformações irreversíveis aparecem quando $\dot{\varepsilon}^{\mathbf{p}} \neq \mathbf{0}$. A deformação plástica acumulada num certo intervalo de tempo $\left[\mathbf{t}_{\mathbf{1}}, \mathbf{t}_{\mathbf{2}}\right]$ é dada por: $\varepsilon^{\mathrm{p}}=\int_{\mathbf{t}_{1}}^{\mathrm{t}_{2}} \dot{\varepsilon}^{\mathrm{p}} \mathbf{d t}$. O significado de tempo, nesse caso, não é o físico, mas a representação da 'história' do carregamento, isto é, cada instante representa um incremento de carga. Do mesmo modo, pode-se dizer que :

$$
\dot{\sigma}=\mathbf{E} \dot{\varepsilon}^{\mathrm{e}}=\mathbf{E}\left(\dot{\varepsilon}-\dot{\varepsilon}^{\mathrm{p}}\right)
$$

A equação acima, escrita em termos de taxas, pode ser escrita também, de modo equivalente, em termos de incrementos, isto é:

$$
\Delta \sigma=\mathbf{E} \Delta \varepsilon^{\mathrm{e}}=\mathbf{E}\left(\Delta \varepsilon-\Delta \varepsilon^{\mathrm{p}}\right)
$$

Considerando-se um comportamento bilinear, isto é, simétrico em tração e compressão, deve-se definir $\dot{\lambda}$ como sendo o valor absoluto da velocidade de deformação plástica $\dot{\varepsilon}$, ou seja:

$$
\dot{\varepsilon}^{\mathbf{p}}=\dot{\lambda} \quad \text { se } \sigma>\mathbf{0}
$$




$$
\dot{\varepsilon}^{\mathbf{p}}=-\dot{\lambda} \quad \text { se } \quad \sigma<\mathbf{0}
$$

Logo, considerando-se as equações (6.6), pode-se dizer que:

$$
\dot{\varepsilon}^{p}=\dot{\lambda} \operatorname{sign}(\sigma)
$$

onde $\operatorname{sign}(\sigma)$ é o sinal da tensão a que o ponto está submetido.

Considerando-se que não se admite $\mathbf{f}(\sigma)_{\mathbf{t}+\Delta \mathbf{t}}>\mathbf{0}$ e que no caso em que $\mathbf{f}(\sigma)_{\mathbf{t}+\Delta \mathbf{t}}<\mathbf{0}$, tem-se um descarregamento, pode-se dizer que:

$$
\begin{aligned}
& \dot{\lambda}=\mathbf{0} \quad \text { se } \mathbf{f}(\sigma)_{\mathbf{t}+\Delta \mathrm{t}}<\mathbf{0} \\
& \dot{\lambda} \geq \mathbf{0} \quad \text { se } \mathbf{f}(\sigma)_{\mathbf{t}+\Delta t}=\mathbf{0}
\end{aligned}
$$

Observando-se as condições anteriores, pode-se dizer que:

$$
\dot{\lambda} \mathbf{f}(\sigma)=\mathbf{0}
$$

que é denominada condição de complementariedade.

A fim de ilustrar os casos de evolução ou não da deformação plástica, considere um certo instante $\mathbf{t}$, onde $\mathbf{f}(\sigma)_{\mathbf{t}}=\mathbf{0}$, como é mostrado na figura (6.2). Nessa situação, deve valer $\dot{\mathbf{f}} \leq \mathbf{0}$ pois $\dot{\mathbf{f}}>\mathbf{0}$ implica em $\mathbf{f}(\sigma)_{\mathbf{t}+\Delta \mathbf{t}}>\mathbf{0}$, o que é inadmissível. Se $\dot{\mathbf{f}}<\mathbf{0}$ (caminho 1), tem-se uma situação de descarregamento e portanto, não há evolução da deformação plástica $(\dot{\lambda}=\mathbf{0})$, mas se $\dot{\mathbf{f}}=\mathbf{0}$ (caminho 2), tem-se evolução da deformação plástica $(\dot{\lambda}>\mathbf{0})$.

Seja agora um certo instante $\mathbf{t}$, onde $\mathbf{f}(\sigma)_{\mathbf{t}}<\mathbf{0}$. Nessa situação, o ponto pode ser carregado até o seu limite elástico, sem haver evolução da deformação plástica. Desse modo, no caso em que o ponto for submetido a uma tensão exatamente igual ao seu limite elástico, ter-se-á $\mathbf{f}(\sigma)_{\mathbf{t}+\Delta t}=\mathbf{0}$ e $\dot{\lambda}=\mathbf{0}$. Caso o ponto seja submetido a uma tensão maior que o seu limite elástico, ele sofrerá descarregamento, haverá evolução da deformação plástica e no final da iteração ter-se á $\mathbf{f}(\sigma)_{\mathbf{t}+\Delta t}=\mathbf{0}$ e $\dot{\lambda}>\mathbf{0}$. 


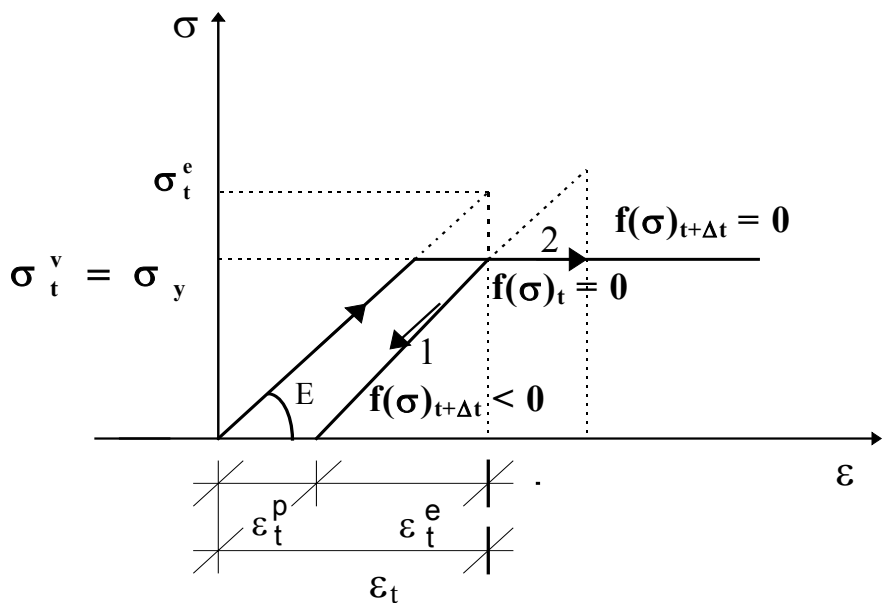

FIGURA 6.2 - Modelo Elasto-plástico Perfeito

onde: $\quad \sigma_{\mathrm{t}}^{\mathrm{e}}$ é tensão suposta totalmente elástica no instante $\mathbf{t}$,

$\sigma_{t}^{\mathrm{v}}$ é tensão verdadeira no instante $\mathbf{t}$,

$\mathbf{f}(\sigma)_{t+\Delta t}$ é o valor do critério correspondente ao instante $\mathbf{t}+\Delta t$.

Assim, pode-se dizer que:

$\dot{\lambda}=\mathbf{0}$ se $\mathbf{f} \leq \mathbf{0}$ e $\dot{\mathbf{f}}<\mathbf{0}$ ( caso de descarregamento )

$\dot{\lambda}>\mathbf{0}$ se $\mathbf{f}=\mathbf{0}$ e $\dot{\mathbf{f}}=\mathbf{0}$ ( caso de carregamento )

Logo, conclui-se que:

$$
\dot{\lambda} \dot{\mathbf{f}}=\mathbf{0},
$$

que é denominada de condição de consistência.

Pode-se obter o valor de $\dot{\lambda}$, através da condição de consistência (6.11). Assim, temse:

$$
\begin{aligned}
\dot{\mathbf{f}} & =\frac{\partial \mathbf{f}}{\partial \mathbf{t}}=\frac{\partial \mathbf{f}}{\partial \sigma} \frac{\partial \sigma}{\partial \mathbf{t}}=\frac{\partial \mathbf{f}}{\partial \sigma} \dot{\sigma}=\frac{\partial \mathbf{f}}{\partial \sigma} \mathbf{E}\left(\dot{\varepsilon}-\dot{\varepsilon}^{\mathrm{p}}\right)=\frac{\partial \mathbf{f}}{\partial \sigma} \mathbf{E}(\dot{\varepsilon}-\dot{\lambda} \operatorname{sign}(\sigma)) \\
\operatorname{mas} \frac{\partial \mathbf{f}}{\partial \sigma} & =\frac{\partial|\sigma|}{\partial \sigma}=\operatorname{sign}(\sigma)
\end{aligned}
$$

Portanto, substituindo-se (6.13) em (6.12), obtém-se: 


$$
\dot{\mathbf{f}}=\frac{\partial \mathbf{f}}{\partial \mathbf{t}}=\operatorname{sign}(\sigma) \mathbf{E} \dot{\varepsilon}-\dot{\lambda} \mathbf{E}[\operatorname{sign}(\sigma)]^{2}
$$

Da relação de consistência tem-se que $\dot{\lambda}>\mathbf{0}$ para $\dot{\mathbf{f}}=\mathbf{0}$. Logo, fazendo $\dot{\mathbf{f}}=\mathbf{0}$ na equação (6.14), obtém-se o valor de $\dot{\lambda}$, para o caso uniaxial:

$$
\dot{\lambda}=\dot{\varepsilon} \cdot \operatorname{sign}(\sigma)
$$

Vale portanto, a relação $\dot{\varepsilon}^{\mathrm{p}}=\dot{\lambda} \operatorname{sign}(\sigma)=\dot{\varepsilon}$ para $\mathbf{f}(\sigma)=\mathbf{0}$ e $\dot{\mathbf{f}}=\mathbf{0}$, isto é, no escoamento, todo acréscimo de deformação é plástico.

\subsubsection{Modelo Elasto-Plástico com Encruamento Isótropo Positivo}

Nesse modelo, o limite elástico inicial se expande, com a evolução da plastificação. Isso se dá de maneira simétrica em relação ao centro do intervalo inicial, ou seja, o centro do intervalo permanece inalterado, mas os limites elásticos de tração e compressão se expandem simetricamente (ver figuras 6.3 e 6.6), mantendo-se as características iniciais de isotropia do material. É adequado para situações de carregamento crescentes, como é o caso apresentado nesse trabalho. No caso de encruamento cinemático, também há evolução dos limites elásticos, mantendo-se a forma e a dimensão da superfície, porém o centro do intervalo sofre translação.

\section{* CASO UNIAXIAL}

Será o modelo considerado para os pontos relativos às armaduras. Assim, será adotado a curva bilinear, isto é, um comportamento simétrico em tração e em compressão, com endurecimento linear do material após escoamento, como é mostrado na figura (6.3), que mostra um ciclo completo de tensão. $O$ trecho 1 representa a fase elástica; no trecho 2, o material foi submetido a uma tensão de tração maior que o limite elástico, ocorrendo encruamento e evolução da deformação plástica ao final do descarregamento. Na fase 3, após o descarregamento, o material é carregado elasticamente, no sentido inverso, até o novo limite elástico de compressão $\left(-\sigma_{\mathbf{y}}^{\text {enc }}\right)$. $\mathrm{Na}$ fase $\mathbf{4}$, o mesmo é submetido a uma tensão maior que $-\sigma_{\mathbf{y}}^{\text {enc }}$, ocorrendo um novo encruamento e nova evolucão da deformação plástica, que é 
igual à anterior em valor absoluto, mas de sinal contrário. Assim, no final do ciclo, tem-se uma deformação plástica acumulada igual a zero.

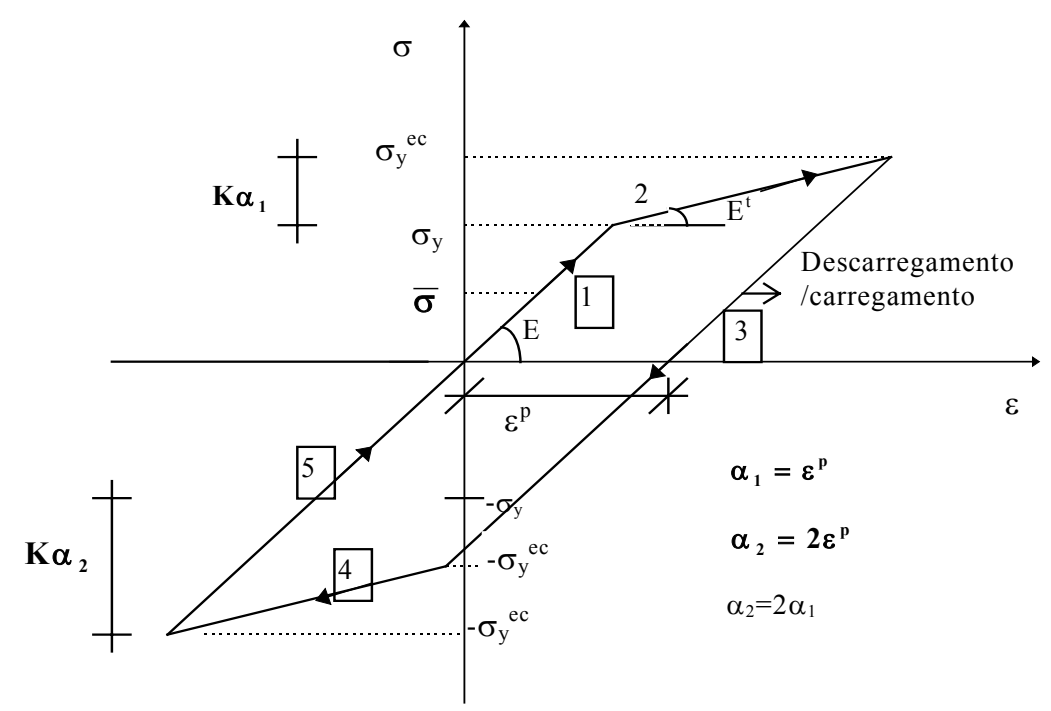

FIGURA 6.3 - Caso Uniaxial de Encruamento Linear Isótropo

O critério de plastificação, nesse caso, é dado por :

$$
\mathbf{f}(\sigma, \alpha)=|\sigma|-\left(\sigma_{\mathbf{y}}+\mathbf{K} \alpha\right) \leq \mathbf{0}
$$

onde:

- K é denominado módulo plástico, ou parâmetro de endurecimento, dado pela tangente à $\operatorname{curva}\left(\sigma \mathbf{x} \varepsilon^{\mathbf{p}}\right)$,

- $\alpha$ é a variável interna associada ao encruamento, que é dada por: $\dot{\alpha}=\left|\dot{\varepsilon}^{\mathrm{p}}\right|$, ou seja, $\dot{\alpha}=\dot{\lambda}$, no caso da hipótese de encruamento por deformação, ou strain hardening

- $\sigma_{\mathbf{y}}^{\mathbf{n}}=\left(\sigma_{\mathbf{y}}+\mathbf{K} \alpha\right)$ representa a tensão de escoamento na iteração $\mathbf{n}$, depois que o material tenha sofrido encruamento.

Considerando-se as equações de $\dot{\varepsilon}^{\mathbf{p}}$, dada por (6.7), e de $\dot{\sigma}$, dada por (6.4), e, ainda, que $\dot{\alpha}=\dot{\lambda}$, a expressão para $\dot{\mathbf{f}}$ é dada por:

$$
\dot{\mathbf{f}}=\frac{\partial \mathbf{f}}{\partial \sigma} \dot{\sigma}+\frac{\partial \mathbf{f}}{\partial \alpha} \dot{\alpha}=\operatorname{sign}(\sigma) \mathbf{E} \dot{\varepsilon}-\dot{\lambda}(\mathbf{E}+\mathbf{K})
$$


Aqui também valem as condições de consistência (6.11) e complementariedade (6.9), mostradas no item anterior. Impondo-se $\dot{\mathbf{f}}=\mathbf{0}$, a condição de consistência garante que $\dot{\lambda}>\mathbf{0}$ e portanto:

$$
\dot{\lambda}=\dot{\alpha}=\frac{\operatorname{sign}(\sigma) \mathbf{E} \dot{\varepsilon}}{(\mathbf{E}+\mathbf{K})}
$$

$\mathrm{O}$ valor de $\Delta \lambda$ é função do incremento de tensão que ultrapassa o limite de escoamento, ou seja, o valor do critério no início da iteração considerada. Portanto, a equação anterior é equivalente à :

$$
\dot{\lambda}=\dot{\alpha}=\frac{\mathbf{f}(\sigma)^{\mathrm{a}}}{(\mathbf{E}+\mathbf{K})}
$$

onde $\mathbf{f}(\sigma)^{\mathbf{a}}$ é o valor do critério no início da iteração

A tensão plástica é dada por:

$$
\dot{\sigma}^{\mathbf{p}}=\mathbf{E} \dot{\varepsilon}^{\mathbf{p}}=\mathbf{E} \dot{\lambda} \sin (\sigma)
$$

Assim, o modelo elasto-plástico uniaxial com encruamento isótropo é dado por:

$$
\dot{\sigma}=\left\{\begin{array}{cc}
\mathbf{E} \dot{\varepsilon} & \text { se } \dot{\lambda}=0 \\
\frac{\mathbf{E K}}{(\mathbf{E}+\mathbf{K})} \dot{\varepsilon} & \text { se } \dot{\lambda} \neq 0
\end{array}\right.
$$

$$
\text { onde } \mathbf{E}^{\mathbf{t}}=\frac{\mathbf{E K}}{(\mathbf{E}+\mathbf{K})}
$$

é o módulo elasto-plástico tangente.

Assim, analogamente ao que foi mostrado na figura (6.2), para o caso elasto-plástico perfeito, em um dado incremento $\mathbf{n}+\mathbf{1}$, o ponto poderá sofrer carregamento plástico (caminho 2 da figura 6.4), isto é, o ponto é submetido a uma tensão maior que o limite elástico; ou sofrer descarregamento elástico (caminho 1 ), onde o ponto é submetido a uma tensão menor à da iteração anterior. 


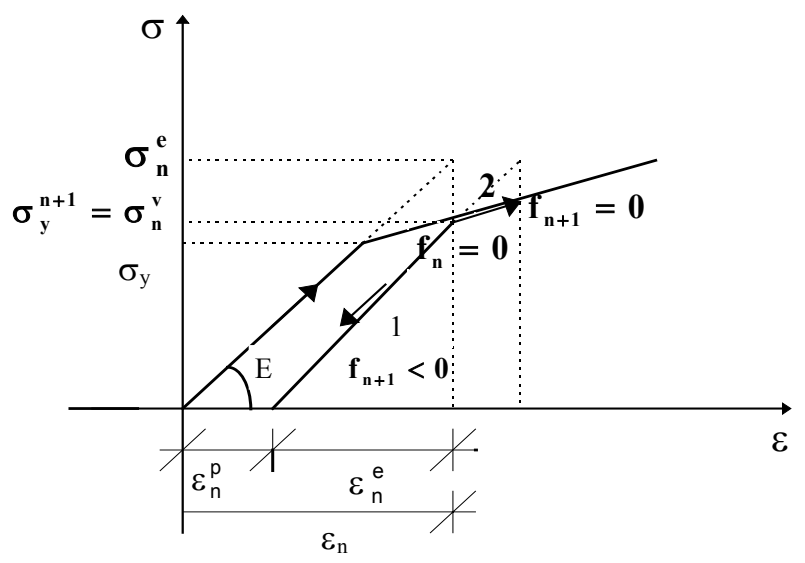

FIGURA 6.4 - Modelo Elasto-Plástico com Encruamento

Para se obter o valor da tensão verdadeira $\sigma_{\mathrm{n}+1}^{\mathrm{v}}$, procede-se da seguinte maneira:

- Inicialmente, supõe-se que a iteração (n+1) é elástica e portanto, têm-se :

$$
\begin{aligned}
& \varepsilon_{n+1}=\varepsilon_{n}+\Delta \varepsilon_{n+1}^{e} \\
& \sigma_{n+1}^{e}=E\left(\varepsilon_{n+1}-\varepsilon_{n}^{p}\right) \text { ou } \sigma_{n+1}^{e}=\sigma_{n}^{v}+\Delta \sigma_{n+1}^{e} \\
& \varepsilon_{n+1}^{p}=\varepsilon_{n}^{p} \\
& \alpha_{n+1}=\alpha_{n}
\end{aligned}
$$

- Verifica-se o critério: $\mathbf{f}_{\mathbf{n}+1}=\left|\sigma_{\mathrm{n}+1}^{\mathrm{e}}\right|-\left(\sigma_{\mathrm{y}}+\mathbf{K} \alpha_{\mathbf{n}+1}\right) \leq \mathbf{0}$

Condição a ser satisfeita : $\Delta \lambda \mathbf{f}_{\mathbf{n}+1}=\mathbf{0}$

$\Rightarrow$ Se $\mathbf{f}_{\mathrm{n}+1} \leq \mathbf{0}$, tem-se que $\Delta \lambda=\mathbf{0}$ e portanto: $\sigma_{\mathrm{n}+1}^{\mathrm{v}}=\sigma_{\mathrm{n}+1}^{\mathrm{e}}$

$\Rightarrow$ Se $\mathbf{f}_{\mathbf{n}+1}>\mathbf{0}$, tem-se que $\Delta \lambda>\mathbf{0}$. Portanto, deve-se procurar um novo estado de tensão tal que $\mathbf{f}_{\mathbf{n}+1}=\mathbf{0}$, como está mostrado na figura (6.5). Calcula-se então $\Delta \lambda$, através da equação (6.19) e o incremento de deformação plástica $\Delta \varepsilon_{\mathbf{n}+1}^{\mathbf{p}}$, com a equação (6.7). Atualiza-se, então, os valores de $\varepsilon_{n+1}^{p}$ e $\alpha_{n+1}: \varepsilon_{n+1}^{p}=\varepsilon_{n}^{p}+\Delta \varepsilon_{n+1}^{p}$ e $\alpha_{n+1}=\alpha_{n}+\Delta \lambda$. A tensão verdadeira $\sigma_{\mathbf{n}+1}^{\mathrm{v}}$ é dada por:

$$
\sigma_{n+1}^{v}=E\left(\varepsilon_{n+1}-\varepsilon_{n+1}^{p}\right)
$$

$\mathrm{ou}$ 


$$
\sigma_{n+1}^{v}=\sigma_{y}^{n+1}+\Delta \sigma_{n+1}^{v}=\sigma_{n}^{v}+\Delta \sigma_{n+1}^{e}-\Delta \sigma_{n+1}^{p}
$$

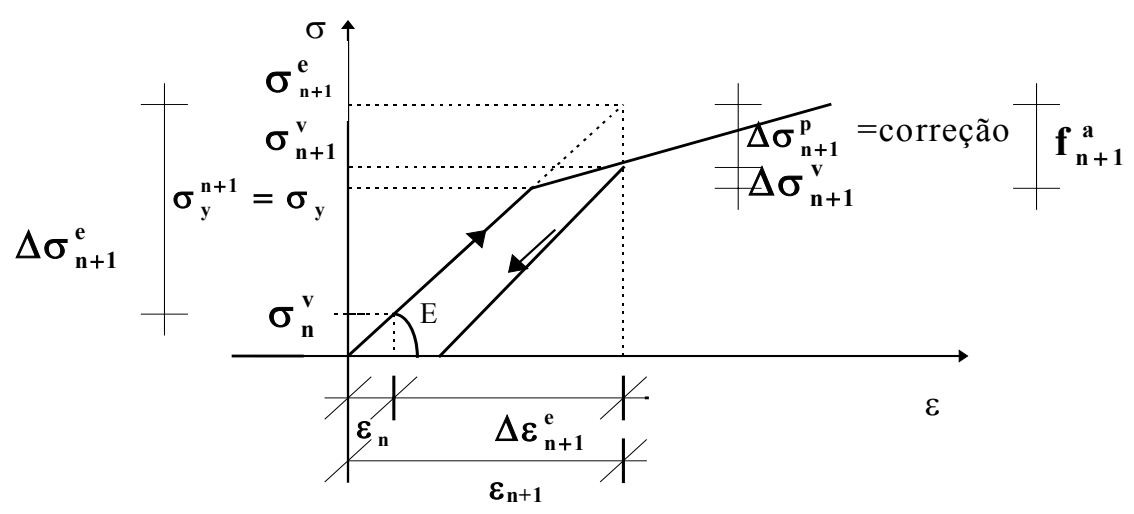

FIGURA 6.5 - Correção da Tensão, num Modelo Elasto-plástico Uniaxial com Encruamento Isótropo Positivo

onde: $\Delta \sigma^{\mathrm{e}}$ é o incremento de tensão suposto elástico,

$\sigma^{\mathrm{e}}$ é a tensão total suposta elástica,

$\sigma^{\mathrm{v}}$ é a tensão verdadeira,

$\Delta \sigma^{\mathrm{p}}$ é a parcela plástica de $\Delta \sigma^{\mathrm{e}}$, ou o excesso de tensão,

$\Delta \sigma^{\mathrm{v}}$ é o incremento verdadeiro de tensão,

$\mathbf{f}_{\mathbf{t}+\Delta \mathbf{t}}^{\mathbf{a}}$ é o valor do critério no início da iteração correspondente ao instante $\mathbf{t}+\Delta \mathbf{t}$.

\section{* CASO MULTIAXIAL}

Esse será um dos modelos utilizados para a placa de concreto. O critério de plastificação, ou de escoamento, no caso bidimensional ou tridimensional, é dado por:

$$
\mathbf{f}(\bar{\sigma}, \mathbf{p})=\bar{\sigma}-\sigma_{\mathbf{y}}(\mathbf{p}) \leq \mathbf{0}
$$

onde:

- $\bar{\sigma}$ é a tensão efetiva, isto é, a tensão equivalente ao estado uniaxial, que é calculada a partir do critério adotado, que, no caso desse trabalho, será o Von Mises, com resistência somente à compressão,

- $\sigma_{\mathbf{y}}(\mathbf{p})$ é a nova tensão de plastificação, após o encruamento, dada por: 


$$
\sigma_{\mathrm{y}}(\mathbf{p})=\left(\sigma_{\mathrm{y}}-\mathbf{p}\right)
$$

- $\mathbf{p}=-\lambda \mathbf{h}(\sigma, \mathbf{p})$

é a variável relacionada ao encruamento isótropo, que, nesse caso, é dada por:

$$
\mathbf{p}=-\mathbf{K} \bar{\varepsilon}^{\mathbf{p}}
$$

- $\bar{\varepsilon}^{\mathbf{p}}$ é a deformação plástica efetiva, ou seja, a deformação plástica equivalente ao estado uniaxial,

- $\sigma_{y}$ é a tensão inicial de escoamento.

No caso multiaxial, o critério de plastificação será representado por uma superfície de plastificação (ver figura 6.6). Assim, em uma dada iteração $\mathbf{n}+\mathbf{1}$, pode-se ocorrer três casos :

-Se $\dot{\mathbf{f}}<\mathbf{0}$, tem-se descarregamento elástico e o ponto está dentro da superfície de plastificação.

-Se $\dot{\mathbf{f}}=\mathbf{0}$, pode-se ter $\dot{\lambda}>\mathbf{0}$ ou $\dot{\lambda}=\mathbf{0}$. Na primeira possibilidade, tem-se carregamento plástico (o ponto cai fora da superfície de plastificação, quando soma-se o incremento de tensão elástico à tensão verdadeira da iteração anterior), com evolução da deformação plástica e do encruamento; e na segunda, tem-se um carregamento neutro, que ocorre em materiais perfeitamente plásticos, onde não há evolução da deformação plástica, ou seja, tem-se $\mathbf{f}=\mathbf{0}$ ao final de dois incrementos consecutivos, sem ocorrer encruamento no último incremento considerado, ou seja, a superfície de plastificação permanece inalterada e o ponto 'anda' sobre a mesma.

A decomposição do tensor de deformações é dada por :

$$
\varepsilon_{\mathrm{ij}}=\varepsilon_{\mathrm{ij}}^{\mathrm{e}}+\varepsilon_{\mathrm{ij}}^{\mathrm{p}}
$$

Ou em termos de taxas:

$$
\dot{\varepsilon}_{\mathrm{ij}}=\dot{\varepsilon}_{\mathrm{ij}}^{\mathrm{e}}+\dot{\varepsilon}_{\mathrm{ij}}^{\mathrm{p}}
$$

A lei de plastificação, ou regra de fluxo, é expressa por :

$$
\dot{\varepsilon}_{\mathrm{ij}}^{\mathrm{p}}=\dot{\lambda} \mathbf{r}(\sigma, \alpha)
$$


onde $\dot{\lambda}$ é o multiplicador plástico e $\mathbf{r}$, que é dado por:

$$
\mathbf{r}=\frac{\partial \mathbf{Q}}{\partial \sigma_{\mathrm{ij}}}
$$

representa o gradiente de tensão do potencial plástico $\mathbf{Q}$ e estabelece a 'direção' do fluxo plástico.

$\mathrm{Na}$ fase elástica a lei incremental é dada por:

$$
\mathbf{d} \sigma_{\mathrm{ij}}=\mathbf{C}_{\mathrm{ijkl}} \mathbf{d} \varepsilon_{\mathrm{kl}}^{\mathrm{e}}
$$

onde $\mathbf{C}_{\mathrm{ijkl}}$ é o tensor de constantes elásticas para materiais isótropos.

Na fase plástica, o caso multiaxial é associado ao caso uniaxial através da tensão e da deformação plástica efetivas. Assim, a lei incremental é dada por:

$$
\mathbf{d} \bar{\sigma}=\mathbf{K} \mathbf{d} \bar{\varepsilon}^{\mathbf{p}}
$$

A relação elasto-plástica entre tensão e deformação é dada por:

$$
\sigma_{\mathrm{ij}}=\mathbf{C}_{\mathrm{ijkl}}\left(\varepsilon_{\mathrm{kl}}-\varepsilon_{\mathrm{kl}}^{\mathrm{p}}\right)=\mathbf{C}_{\mathrm{ijkl}}^{\mathrm{ep}} \varepsilon_{\mathrm{kl}}
$$

onde $\mathbf{C}^{\text {ep }}$ é o tensor dos módulos elasto-plásticos tangentes.

Uma expressão para $\dot{\lambda}$ pode ser obtida da relação para $\dot{\mathbf{f}}$ :

$$
\dot{\mathbf{f}}=\mathbf{f}_{\sigma} \cdot \dot{\sigma}+\mathbf{f}_{\mathbf{p}} \cdot \dot{\mathbf{p}}=\mathbf{f}_{\sigma} \cdot \mathbf{C} \dot{\varepsilon}-\dot{\lambda}\left(\mathbf{f}_{\sigma} \cdot \mathbf{C r}+\mathbf{f}_{\mathbf{p}} \mathbf{h}\right)
$$

Considerando-se a lei de consistência, $\dot{\lambda}>\mathbf{0}$ só é possível se $\dot{\mathbf{f}}=\mathbf{0}$. Logo, igualando a equação (6.34) a zero, obtém-se:

$$
\dot{\lambda}=\frac{f_{\sigma} \cdot C \dot{\varepsilon}}{f_{\sigma} \cdot C r+f_{p} h}
$$

Substituindo-se as equações (6.35) e (6.29) em (6.33), obtém-se $\mathbf{C}^{\text {ep }}$ : 


$$
C^{\text {ep }}=\left\{\begin{array}{cc}
C & \text { se } \dot{\lambda}=0 \\
C-\frac{\left(\mathrm{Cr} \otimes \mathbf{C f}_{\sigma}\right)}{\mathbf{f}_{\sigma} \mathbf{C r}+\mathbf{f}_{\mathrm{p}} \mathbf{h}} & \text { se } \dot{\lambda}>0
\end{array}\right.
$$

Considere um certo instante $\mathbf{t}$, onde ocorreu uma mudança incremental na função de escoamento, dada por $\mathbf{d f}=\frac{\partial \mathbf{f}}{\partial \sigma_{\mathrm{ij}}} \mathbf{d} \sigma_{\mathrm{ij}}=\mathbf{f}_{\sigma} \mathbf{d} \sigma$, devida a um incremento de tensão. Considerando-se que $\mathbf{d} \sigma$ é tangente à superfície, conclui-se que $\mathbf{f}_{\sigma}$ deve ser normal à superfície para que se tenha $\mathbf{f}_{\mathbf{t}}=\mathbf{0}$.

Para um $\mathbf{r}$ arbitrário $\mathbf{C}^{\mathbf{e p}}$ é, em geral, não-simétrico. A simetria é recuperada quando faz-se $\mathbf{r}=\mathbf{f}_{\sigma}$ (equação 6.30), ou seja, o potencial plástico $\mathbf{Q}$ coincide com a função de escoamento f. Nesse caso, a lei de fluxo é dita associativa. Essa situação implica na chamada regra da normalidade, pois o tensor taxa de deformação plástica passa a ter a direção da normal à superfície de plastificação, ou seja, $\dot{\varepsilon}^{\mathbf{p}}=\dot{\lambda} \mathbf{f}_{\sigma}$.

Admitindo-se na equação (6.35) que $\mathbf{f}_{\boldsymbol{\sigma}} \cdot \mathbf{C r}+\mathbf{f}_{\mathbf{p}} \mathbf{h}>\mathbf{0}$, ter-se-á $\dot{\lambda} \geq \mathbf{0}$ somente se $\mathbf{f}_{\sigma} \cdot \mathbf{C} \dot{\boldsymbol{\varepsilon}} \geq \mathbf{0}$. Tendo-se em vista que $\mathbf{f}_{\sigma}$ é normal à superfície, conclui-se que o ângulo entre $\mathbf{f}_{\sigma}$ e $\mathbf{C} \dot{\boldsymbol{\varepsilon}}$ deve ser menor ou igual a $90^{\circ}$. Considerando-se que $\mathbf{r}=\mathbf{f}_{\sigma}$, a hipótese $\mathbf{f}_{\sigma} \mathbf{C r}+\mathbf{f}_{\mathbf{p}} \mathbf{h}>\mathbf{0}$, só é verificada para $\mathbf{h}=\mathbf{K} \mathbf{f}_{\mathbf{p}}$, tendo em vista que $\mathbf{C}$ é definido positivo.

Assim, considerando-se a lei associativa e a relação $\mathbf{h}=\mathbf{K} \mathbf{f}_{\mathbf{p}}$, as equações (6.26.a), (6.29), (6.35) e (6.36) resultam em:

$$
\begin{aligned}
& \dot{\varepsilon}_{\mathrm{ij}}^{\mathrm{p}}=\dot{\lambda} \mathbf{f}_{\sigma} \\
& \dot{\mathbf{p}}=-\dot{\lambda} \mathbf{K} \mathbf{f}_{\mathbf{p}}=-\dot{\lambda} \mathbf{K} \\
& \dot{\lambda}=\frac{\mathbf{f}_{\sigma} \mathbf{C} \dot{\varepsilon}}{\mathbf{f}_{\sigma} \mathbf{C} f_{\sigma}+K} \\
& \mathrm{C}^{\text {ep }}=\left\{\begin{array}{cc}
\mathrm{C} & \text { se } \dot{\lambda}=0 \\
\mathrm{C}-\frac{\left(\mathbf{C f _ { \sigma } \otimes C f _ { \sigma } )}\right.}{\mathbf{f}_{\sigma} \mathbf{C f _ { \sigma }}+K} & \text { se } \dot{\lambda}>0
\end{array}\right.
\end{aligned}
$$


Assim, considerando-se um algoritmo implícito no cálculo das tensões, o critério de plastificação de Von-Mises, a lei da normalidade, e o encruamento isótropo positivo, pode-se dar uma interpretação geométrica ao problema no sistema principal, que é mostrada na figura (6.6). No caso de um algoritmo implícito, a correção do estado de tensões é feita segundo a normal à superfície na posição atual $(\mathbf{n}+\mathbf{1})$ e num algoritmo explícito, a mesma é feita segundo a normal à superfície da iteração anterior (n).

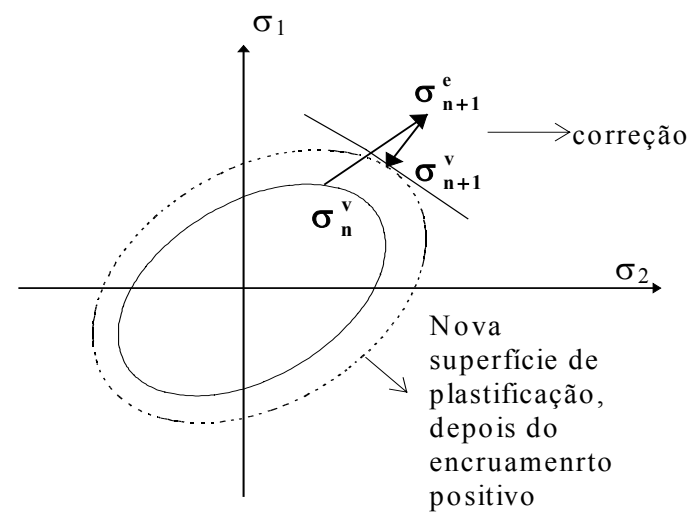

FIGURA 6.6 - Representação Geométrica do Critério de Von Mises no caso biaxial

onde: $\sigma_{\mathbf{n}}^{\mathrm{v}}$ é o estado de tensão verdadeiro do ponto, na iteração $\mathbf{n}$,

$\sigma_{\mathbf{n}+\mathbf{1}}^{\mathrm{e}}$ é o estado de tensão, suposto elástico, na iteração $\mathbf{n}+\mathbf{1}$,

$\sigma_{\mathbf{n}+1}^{\mathrm{v}}$ é o estado de tensão verdadeiro do ponto, na iteração $\mathbf{n}+\mathbf{1}$.

Escrevendo-se as relações (6.37), (6.39) e (6.40) através da notação indicial e considerando-se:

$$
\mathbf{f}_{\sigma}=\frac{\partial \mathbf{f}\left(\sigma_{\mathrm{ij}}\right)}{\partial \sigma_{\mathrm{ij}}}=\frac{\partial \bar{\sigma}}{\partial \sigma_{\mathrm{ij}}}=\mathbf{a}_{\mathrm{ij}}
$$

e

$$
\mathbf{d}_{\mathrm{ij}}=\mathbf{a}_{\mathrm{ij}} \mathbf{C}_{\mathrm{ijkl}} \text {, }
$$

obtêm-se:

$$
\dot{\varepsilon}_{\mathrm{ij}}^{\mathrm{p}}=\dot{\lambda} \mathbf{a}_{\mathrm{ij}}
$$




$$
\dot{\lambda}=\frac{\mathbf{a}_{\mathrm{ij}} \mathbf{C}_{\mathrm{ijkl}} \dot{\varepsilon}_{\mathrm{kl}}}{\mathbf{a}_{\mathrm{ij}} \mathbf{d}_{\mathrm{ij}}+\mathbf{K}}=\frac{\mathbf{a}_{\mathrm{ij}} \dot{\sigma}_{\mathrm{ij}}}{\mathbf{a}_{\mathrm{ij}} \mathbf{d}_{\mathrm{ij}}+\mathbf{K}}
$$

sendo $\dot{\sigma}_{\mathrm{ij}}$ o incremento de tensão acima do limite elástico.

$$
C^{\text {ep }}=\left\{\begin{array}{cc}
C_{i j k l} & \text { se } \dot{\lambda}=0 \\
C_{i j k l}-\frac{\left(d_{i j} a_{m n} C_{m n k l}\right)}{a_{m n} d_{m n}+K} & \text { se } \dot{\lambda}>0
\end{array}\right.
$$

Assim, o incremento verdadeiro de tensão é dado por:

$$
\mathbf{d} \sigma_{\mathrm{ij}}^{\mathrm{v}}=\mathbf{d} \sigma_{\mathrm{ij}}^{\mathrm{e}}-\mathbf{d} \sigma_{\mathrm{ij}}^{\mathrm{p}}
$$

onde: $\mathbf{d} \sigma_{\mathrm{ij}}^{\mathrm{e}}=\mathbf{C}_{\mathrm{ijkl}} \mathbf{d} \varepsilon_{\mathrm{kl}}$

$$
d \sigma_{i j}^{p}=\frac{d_{i j} a_{m n}}{a_{m n} d_{m n}+K} d \sigma_{m n}^{e}=d_{i j} d \lambda
$$

A lei de evolução do encruamento é dada em função do trabalho plástico $\mathbf{W}_{\mathbf{p}}$ realizado durante as deformações, segundo a hipótese de work hardening:

$$
\Delta \mathbf{W}_{\mathrm{p}}=\Delta \bar{\varepsilon}_{\mathrm{n}+1}^{\mathrm{p}} \bar{\sigma}_{\mathrm{n}+1}^{\mathrm{v}}=\Delta \varepsilon_{\mathrm{ij}(\mathrm{n}+1)}^{\mathrm{p}} \sigma_{\mathrm{ij}(\mathrm{n}+1)}^{\mathrm{v}}
$$

Escrevendo-se a equação (6.43) em termos de incrementos e substituindo-a em (6.49), obtém-se o incremento de deformação plástica efetiva:

$$
\Delta \bar{\varepsilon}_{n+1}^{p}=\frac{\Delta \lambda \mathbf{a}_{\mathrm{ij}} \sigma_{\mathrm{ij}(\mathrm{n}+1)}^{\mathrm{v}}}{\bar{\sigma}_{\mathrm{n}+1}^{\mathrm{v}}}
$$

O Teorema de Euler para funções homogêneas diz que se f é uma função homogênea, tem-se que $\mathbf{f}_{\sigma} \cdot \sigma=\mathbf{n} . \mathbf{f}$, onde $\mathbf{n}$ é o grau de homogeneidade da função. Assim, no caso do critério de Von Mises, tem-se que f é uma função de grau unitário, e portanto, podese dizer que $\sigma_{\mathrm{ij}} \mathbf{a}_{\mathrm{ij}}=\mathbf{f}\left(\sigma_{\mathrm{ij}}\right)$. Logo, conclui-se que:

$$
\Delta \bar{\varepsilon}_{n+1}^{P}=\Delta \lambda
$$


e portanto, para os casos em que o parâmetro de endurecimento $\mathbf{K}$ é constante, a equação (6.38) resulta em:

$$
\Delta \mathbf{p}=-\mathbf{K} \Delta \overline{\bar{\varepsilon}}^{\mathbf{p}}
$$

Assim, para se obter a tensão verdadeira $\sigma_{\mathrm{ij}(\mathbf{n}+\mathbf{1})}^{\mathbf{v}}$ em uma determinada iteração $(\mathbf{n}+\mathbf{1})$, procede-se da seguinte maneira:

- Inicialmente, supõe-se que a iteração (n+1) é elástica, portanto têm-se:

$$
\begin{aligned}
& \varepsilon_{\mathrm{ij}(\mathrm{n}+1)}=\varepsilon_{\mathrm{ij}(\mathrm{n})}+\Delta \varepsilon_{\mathrm{ij}(\mathrm{n}+1)}^{\mathrm{e}} \\
& \sigma_{\mathrm{ij}(\mathrm{n}+1)}^{\mathrm{e}}=\mathbf{E}\left(\varepsilon_{\mathrm{ij}(\mathrm{n}+1)}-\varepsilon_{\mathrm{ij}(\mathrm{n})}^{\mathrm{p}}\right)=\sigma_{\mathrm{ij}(\mathrm{n})}^{\mathrm{v}}+\Delta \sigma_{\mathrm{ij}(\mathrm{n}+1)}^{\mathrm{e}} \\
& \bar{\varepsilon}_{\mathrm{n}+1}^{\mathrm{p}}=\bar{\varepsilon}_{\mathrm{n}}^{\mathrm{p}}
\end{aligned}
$$

- Com $\sigma_{\mathrm{ij}(\mathbf{n}+1)}^{\mathrm{e}}$ calcula-se $\bar{\sigma}_{(\mathbf{n}+1)}^{\mathrm{e}}$ de acordo com o critério especificado.

- Verifica-se o critério de plastificação: $\mathbf{f}_{\mathbf{n}+1}=\bar{\sigma}_{\mathbf{n}+1}^{\mathrm{e}}-\left(\sigma_{\mathrm{y}}+\mathbf{K} \bar{\varepsilon}_{\mathbf{n}+1}^{\mathrm{p}}\right) \leq \mathbf{0}$

Condição a ser satisfeita: $\Delta \lambda \mathbf{f}_{\mathbf{n}+1}=\mathbf{0}$.

$\Rightarrow$ Se $\mathbf{f}_{\mathbf{n}+1} \leq \mathbf{0}$, tem-se $\Delta \lambda=\mathbf{0}$ e, portanto: $\sigma_{\mathrm{ij}(\mathbf{n}+1)}^{\mathrm{v}}=\sigma_{\mathrm{ij}(\mathbf{n}+1)}^{\mathrm{e}}$

$\Rightarrow$ Se $\mathbf{f}_{\mathbf{n}+\mathbf{1}}>\mathbf{0}$, tem-se $\Delta \lambda>\mathbf{0}$ e, portanto, deve-se procurar um novo estado de tensão tal que $\mathbf{f}_{\mathbf{n}+\mathbf{1}}=\mathbf{0}$, como está mostrado na figura (6.6). Calcula-se então $\Delta \lambda$, através da equação (6.44), o incremento de deformação plástica $\Delta \varepsilon_{\mathbf{n}+1}^{\mathbf{p}}$, com a equação (6.50), atualizando-se o valor de $\varepsilon_{\mathbf{n}+\mathbf{1}}^{\mathbf{p}}: \varepsilon_{\mathbf{n}+\mathbf{1}}^{\mathbf{p}}=\varepsilon_{\mathbf{n}}^{\mathbf{p}}+\Delta \varepsilon_{\mathbf{n}+1}^{\mathbf{p}}$ e por fim, a tensão verdadeira:

$$
\sigma_{\mathrm{ij}(\mathrm{n}+1)}^{\mathrm{v}}=\sigma_{\mathrm{ij}(\mathrm{n}+1)}^{\mathrm{y}}+\Delta \sigma_{\mathrm{ij}(\mathbf{n}+1)}^{\mathrm{v}}=\sigma_{\mathrm{ij}(\mathbf{n})}^{\mathrm{v}}+\Delta \sigma_{\mathrm{ij}}^{\mathrm{e}}-\Delta \lambda \mathbf{d}_{\mathrm{ij}}
$$

onde $\sigma_{\mathrm{ij}(\mathbf{n}+1)}^{\mathrm{y}}$ são as tensões correspondentes ao limite elástico $\sigma_{\mathbf{y}}^{\mathbf{n}+1}$.

Assim, considerando-se resistência somente à compressão, a superfície de escoamento, representada na figura (6.6), é dada por: 


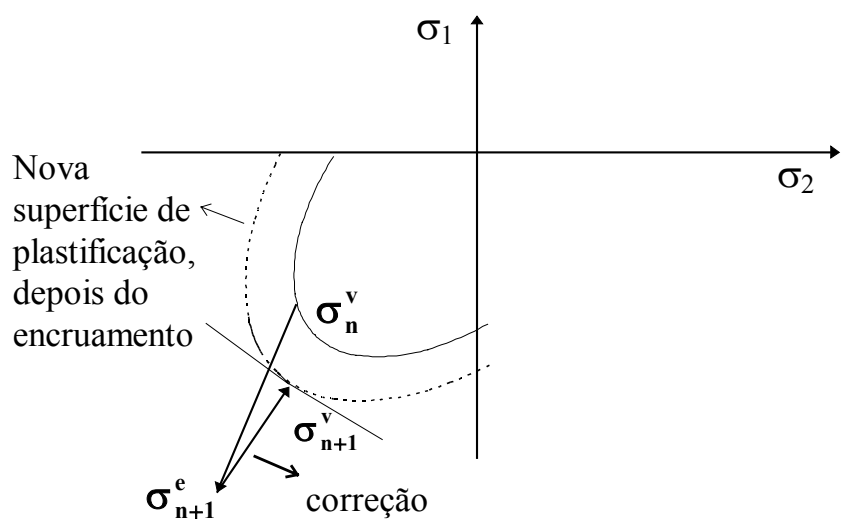

FIGURA 6.7 - Representação Geométrica do Critério de Von Mises no caso biaxial, com Resistência somente à Compressão.

\section{* CRITÉRIO DE VON MISES}

Um critério de escoamento é independente da orientação do sistema de coordenadas empregado, sendo, usualmente, expresso em função dos invariantes de tensão. No critério de Von Mises, o escoamento ocorre quando o segundo invariante atinge um valor $\mathbf{k}$, ou seja, quando:

$$
\sqrt{\mathbf{J}_{2}}=\mathbf{k}
$$

$$
\text { onde: } \mathbf{J}_{\mathbf{2}}=\frac{\mathbf{1}}{\mathbf{2}} \mathbf{S}_{\mathrm{ij}} \mathbf{S}_{\mathrm{ij}} \quad(\mathrm{i}, \mathrm{j}=1,2,3)
$$

sendo $\mathbf{S}_{\mathrm{ij}}$ a parte anti-esférica do vetor de tensões, que é dada por:

$$
\begin{gathered}
\mathbf{S}_{\mathrm{ij}}=\sigma_{\mathrm{ij}}-\delta_{\mathrm{ij}} \sigma_{\mathrm{m}} \\
\text { e } \sigma_{\mathrm{m}}=\frac{\sigma_{\mathrm{ii}}}{3} .
\end{gathered}
$$

Expressando-se (6.55) em função das tensões principais, obtém-se:

$$
\mathbf{J}_{2}=\frac{1}{6}\left[\left(\sigma_{1}-\sigma_{2}\right)^{2}+\left(\sigma_{2}-\sigma_{3}\right)^{2}+\left(\sigma_{3}-\sigma_{1}\right)^{2}\right]
$$

Considerando-se um caso bidimensional $\left(\sigma_{3}=\mathbf{0}\right)$, o critério, que é dado pela equação (6.54), pode ser escrito na forma: 


$$
\sigma_{1}^{2}+\sigma_{2}^{2}-\sigma_{1} \sigma_{2} \leq 3 \mathbf{k}^{2}
$$

que é a equação de uma elipse, como está representado na figura (6.6).

Do ensaio uniaxial, obtêm-se uma relação entre $\mathbf{k}$ e $\sigma_{\mathbf{y}}(\mathbf{p})$, dado pela equação (6.25), e o critério passa a ser:

$$
\mathbf{f}(\bar{\sigma}, \mathbf{p})=\sqrt{\mathbf{3 \mathbf { J } _ { 2 }}}-\sigma_{\mathbf{y}}(\mathbf{p})=\mathbf{0}
$$

Logo, a tensão efetiva é dada por:

$$
\bar{\sigma}=\sqrt{\mathbf{3 J _ { 2 }}}
$$

Escrevendo-se agora a equação (6.55) de $\mathbf{J}_{2}$, para o sistema $\mathbf{x}_{1} \mathbf{x}_{2}$, obtém-se;

$$
\mathbf{J}_{2}=\sigma_{12}^{2}+\frac{1}{2}\left[\left(\sigma_{11}-\sigma_{m}\right)^{2}+\left(\sigma_{22}-\sigma_{m}\right)^{2}+\sigma_{m}^{2}\right]
$$

Desse modo, os valores de $\mathbf{a}_{\mathrm{ij}}$, dados pela equação (6.41), resultam em:

$$
\mathbf{a}_{\mathrm{ij}}=\frac{\partial \mathbf{f}}{\partial \mathbf{J}_{2}} \frac{\partial \mathbf{J}_{2}}{\partial \sigma_{\mathrm{ij}}}=\frac{\sqrt{\mathbf{3}}}{2 \sqrt{\mathbf{J}_{2}}} \frac{\partial \mathbf{J}_{2}}{\partial \sigma_{\mathrm{ij}}}
$$

\section{* MODELO ELASTO-PLÁSTICO COM ENCRUAMENTO ISÓtROPO NEGATIVO}

Esse será um dos modelos utilizados para a placa de concreto. A formulação é idêntica àquela apresentada para o caso multiaxial com encruamento isótropo positivo. A única diferença, é que o módulo plástico $\mathbf{K}$ é definido negativo, ao invés de positivo. Assim, ao invés dos limites elásticos aumentarem de valor com a evolução da deformação plástica, eles diminuem. Graficamente, para o caso uniaxial, considerando-se que não haja resistência à tração, o modelo é representado por: 


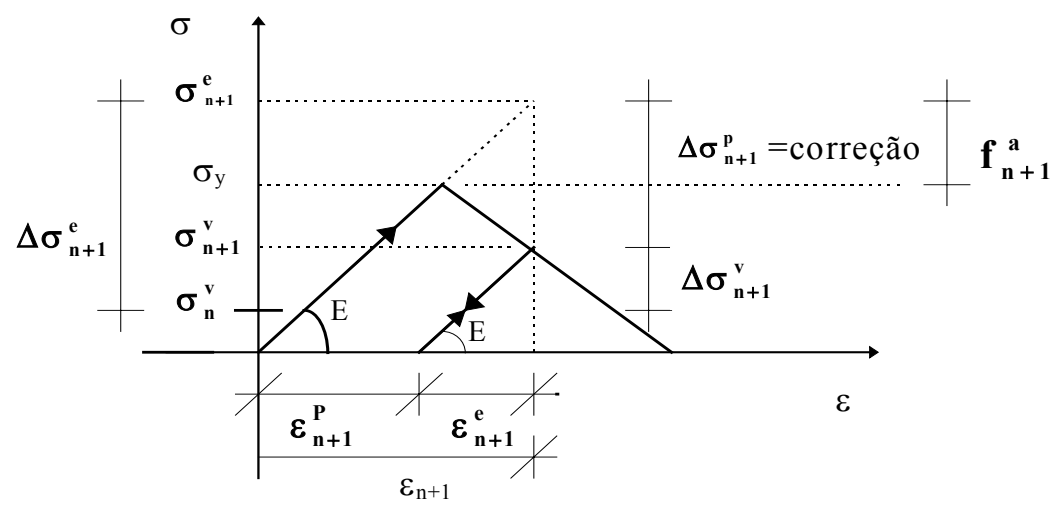

FIGURA 6.8 - Correção das Tensões para o Modelo Uniaxial de Encruamento Isótropo Negativo

\subsection{Modelo de Dano}

\subsubsection{Introdução}

A Mecânica do Dano Contínuo tem sido aplicada na solução de vários problemas dentro da engenharia, aos quais se incluem a modelação da deterioração lenta do material (creep damage) (KACHANOV (1984) e MURAKAMI (1981)), dano por fadiga (LEMAITRE (1984) e MARIGO (1985)), interação deterioração lenta-fadiga (LEMAITRE \& CHABOCHE (1974) e LEMAITRE (1984)) entre outros.

Esse será um outro modelo a ser considerado para o concreto, onde se considera que a ruptura ocorre devido ao processo de abertura de fissuras, que provoca perda nas propriedades mecânicas, isto é, há perda de rigidez e da resistência após-pico. O fenômeno de 
perda de resistência é conhecido como amolecimento ou 'softening' e é representado pela redução do limite elástico; a perda de rigidez é devido à redução do módulo de elasticidade, ou módulo de Young. Assim, esse modelo permite relacionar as alterações na estrutura interna, a nível microscópico, com os fenômenos evidenciados macroscopicamente. Como no caso do concreto a fissuração é o fenômeno dominante que comanda o comportamento não-linear, esse é um bom modelo.

Assim, o modelo de dano, baseado na termodinâmica dos processos irreversíveis, descreve a evolução local do processo de deterioração do material, através de variáveis internas, partindo-se de uma configuração inicial ideal, sem defeitos, até um estado final onde fissuras ou macrofissuras possam ser observadas. O meio deteriorado é tratado como um meio contínuo de rigidez e resistência reduzidas. O dano se inicia com pequenas deformações, desenvolve-se progressivamente atingindo uma taxa máxima logo após o pico de tensão e tende a um valor assintótico com o crescimento da deformação, como é mostrado na figura (6.9), para o caso uniaxial, onde pode-se observar que a resistência do concreto à compressão é aproximadamente dez vezes maior que à tração. A figura (6.9) não é referente a nenhum modelo específico, ela é apresentada apenas com a finalidade de ilustrar o problema.

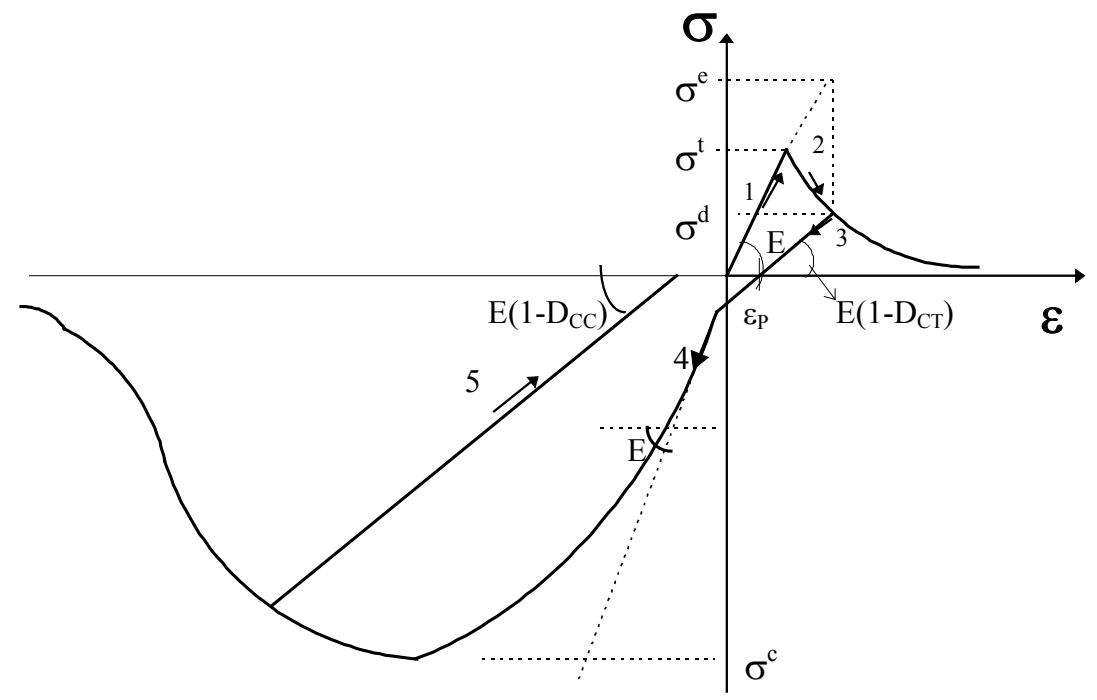

FIGURA 6.9 - Modelo de Dano Uniaxial no Concreto

onde: $\sigma^{\mathbf{d}}$ é o novo limite elástico em tração do material danificado, $\sigma^{\mathrm{e}}$ é a tensão suposta elástica, 
$\sigma^{\mathfrak{c}}$ é o limite elástico inicial em compressão,

$\sigma^{\mathrm{t}}$ é o limite elástico em tração,

E é o módulo de elasticidade inicial,

$\mathbf{E}\left(\mathbf{1}-\mathbf{D}_{\mathbf{C}}\right)$ é o módulo de elasticidade do concreto danificado,

$\mathbf{D}_{\mathbf{C T}}$ é o dano no concreto em tração,

$\mathbf{D}_{\mathbf{C C}}$ é o dano no concreto em compressão.

Na figura (6.9), tem-se a representação de um ciclo de tensão, onde o trecho 1 representa um carregamento elástico em tração. No trecho $\mathbf{2}$, o material foi submetido à uma tensão de tração maior que o limite elástico, ocorrendo evolução do dano e da deformação plástica ao final do descarregamento do trecho 3. Se houver um novo carregamento em tração, ele se dará pela reta $\mathbf{3}$ e o novo limite elástico de tração do material com dano é $\sigma^{\mathbf{d}}$. Após o descarregamento do trecho 3, o material é carregado elasticamente, no sentido inverso, segundo uma rigidez igual ao do trecho 1 (caminho 4) Assim, pode-se observar o comportamento unilateral do concreto, que é traduzido pela recuperação da rigidez inicial quando há inversão no sentido de carregamento, após ter sido submetido a um nível de dano elevado, causado pela tensão de tração. Isso ocorre, porque fissuras previamente abertas se fecham. No trecho 4, o material é carregado elasticamente até uma tensão de compressão maior que o limite elástico, ocorrendo evolução do dano em compressão e da deformação plástica ao final do descarregamento do trecho 5 .

Estudos mostram que a resistência mecânica efetiva do concreto é muito baixa em relação à resistência teórica necessária para vencer a coesão molecular considerando o meio como homogêneo e livre de defeitos. Isso ocorre devido à presença de defeitos internos do material, os quais se apresentam sob a forma de poros, microfissuras ou vazios. Os poros se encontram na pasta de cimento e nos agregados; as microfissuras ocorrem mesmo antes da aplicação dos esforços devido ao fenômeno de retração mais liberação de calor que ocorre na cura e os vazios são causados por insuficiência no adensamento e/ou cura do concreto fresco.

Os modelos de dano podem ser escalares, isótropos, ou anisótropos, dependendo da natureza da variável de dano usada. A variável interna de dano muda de acordo com o modelo considerado; assim, a mesma pode ser função da deformação equivalente, da densidade de vazios, de uma combinação da parte hidróstática e da parte desviadora do tensor de tensões ou de outros. Pode-se ter modelos que envolvam o comportamento unilateral do concreto, outros que consideram a evolução da deformação plástica, onde as mesmas são causadas por microfissurações e deslizamentos plásticos, outros, ainda, onde a 
evolução do dano é devido a uma combinação de mudanças da área e da orientação dos microdefeitos. Pode-se também, ter modelos de dano que combinam a teoria clássica da plasticidade com o modelo de fratura, outros que combinam a teoria da plasticidade e o modelo de fissura localizada. Assim, a escolha do modelo apropriado dependerá do tipo de problema em questão e da precisão requerida, que é função do número de parâmetros a serem identificados.

Nesse trabalho será usado o modelo de MAZARS (1984), que, devido a sua simplicidade, poderá evidenciar o seu uso em conjunto com o Método dos Elementos de Contorno (MEC).

\subsubsection{Formação e Propagação de Fissuras}

A nível microscópico, o concreto é considerado como um material composto de duas fases homogêneas: a pasta de cimento e os agregados. Na zona de contato entre a pasta e o agregado, a pasta é mais porosa e portanto, menos resistente. Onde a ligação é solicitada à compressão, ela se compacta e onde ela é solicitada à tração, ela se rompe mais facilmente que o resto da pasta e o agregado. Assim, as primeiras fissuras aparecerão primeiro na interface pasta/agregado, para depois se propagarem na pasta e nos agregados. Se o agregado apresentar resistência elevada, elas se propagarão primeiro na pasta, se não elas se propagarão primeiro no agregado. A nível macroscópico, o concreto é considerado, no estado inicial, como um material homogêneo e isótropo.

A heterogeneidade do concreto é devido aos seus diferentes constituintes e também à não uniformidade do estado de tensão interno, causada pela presença de vazios, por cargas não uniformes e pelas diferentes orientações das superfícies formadas pela pasta e agregados e entre agregados, em relação à direção de uma força aplicada. Com isso, os níveis de solicitação local podem variar muito em relação ao valor nominal da solicitação aplicada. A presença de vazios favorece a concentração de tensões, sendo pontos favoráveis à formação de fissuras.

Na mecânica da fratura, tem-se três tipos básicos de abertura de fissura:

- Modo I: a abertura de fissura é devido a um esforço perpendicular à superfície do defeito.

- Modo II: há escorregamento das superfícies da fissura devido a um esforço cisalhante aplicado no plano dessas superfícies e paralelo ao defeito.

- Modo III: há escorregamento das superfícies da fissura, devido a um esforço cisalhante que atua no plano dessas superfícies e em direção perpendicular ao defeito 


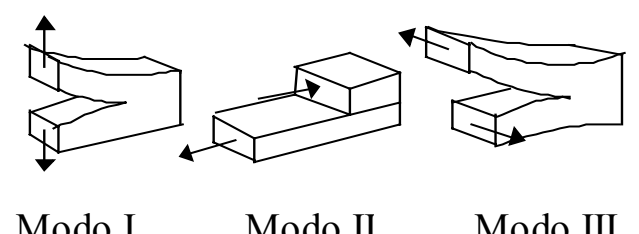

FIGURA 6.10 - Tipos de Abertura de Fissura

A nível microestrutural, observou-se que no caso de tração uniaxial o crescimento dos microdefeitos ocorre em direção perpendicular à da solicitação, isto é em modo I, até a formação de uma microfissura. Na compressão uniaxial, o efeito de Poisson, que é traduzido pelo aumento da resistência quando aumenta-se a menor tensão de compressão num ensaio de tração-compressão biaxial, e a heterogeneidade do material produzem alongamentos e tensões de tração que fazem com que as microfissuras se desenvolvam paralelamente à solicitação, segundo um modo misto de abertura (modo I e II).

As microfissuras crescem predominantemente em modo I, sempre que o material, sujeito a um esforço genérico, apresentar alongamentos. Isso explica o comportamento não simétrico do concreto em tração e compressão.

\subsubsection{Variáveis de Dano}

Seja um sólido danificado do qual é retirado um elemento de volume representativo (Figura 6.11). Tal elemento é pequeno a fim de ser interpretado como um ponto material do contínuo, mas suficientemente grande para incluir as imperfeições e os vários ingredientes do meio.

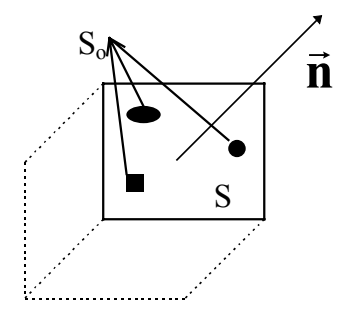

FIGURA 6.11 - Elemento de Volume com Dano

$\mathrm{Na}$ figura (6.11), $\mathbf{S}$ é a área total da seção definida pelo versor $\mathbf{n}$ e $\mathbf{S}_{\mathbf{0}}$ é a área com defeitos. Em S, as microfissuras e microdefeitos que contribuem para o dano têm uma distribuição aleatória. Assim, sendo $\overline{\mathbf{S}}$ a área resistente efetiva, pode-se definir a área com defeitos $\mathbf{S}_{\mathbf{0}}$, como: 


$$
\mathbf{S}_{\mathbf{0}}=\mathbf{S}-\overline{\mathbf{S}}
$$

Através da hipótese de continuidade, pode-se ter uma medida mecânica do dano local $\mathbf{D}_{\mathbf{n}}$, ou densidade superficial do dano, num determinado plano de normal $\mathbf{n}$ e área $\mathbf{S}$, representando o caso de dano anisotrópico, e que é dada por:

$$
D_{n}=\lim _{S \rightarrow 0} \frac{S_{0}}{S}
$$

onde: $\quad \mathbf{0} \leq \mathbf{D}_{\mathbf{n}} \leq \mathbf{1}$

$\mathbf{D}_{\mathbf{n}}=\mathbf{0}$ representa o material íntegro,

$\mathbf{D}_{\mathbf{n}}=\mathbf{1}$ representa o estado limite de dano.

\section{- Hipóteses de Isotropia}

O modelo de dano isótropo é baseado na hipótese de que os microdefeitos têm uma distribuição uniforme no elemento, isto é, a variável de dano é independente da normal $\mathbf{n}$. Assim, o dano é representado por uma variável escalar $\mathbf{D}_{\mathbf{C}}$ e a hipótese de isotropia é dada por:

$$
\mathbf{D}_{\mathrm{n}}=\mathbf{D}_{\mathbf{C}}, \quad \forall \mathbf{n}
$$

\subsubsection{Deformação Equivalente}

Considere, no caso unidimensional de dano isótropo, um corpo em equilíbrio estático, sujeito a uma força $\mathbf{F}$. Pode-se escrever a tensão no mesmo como:

$$
\sigma=\frac{\mathbf{F}}{\mathbf{S}}
$$

A variável de dano isótropo é dada por: 


$$
\mathbf{D}_{\mathrm{C}}=\frac{\mathbf{S}_{0}}{\mathrm{~S}}
$$

Considerando-se a equação (6.68), a área efetiva é dada por:

$$
\overline{\mathbf{S}}=\mathbf{S}-\mathbf{S}_{\mathbf{0}}=\mathbf{S}\left(\mathbf{1}-\mathbf{D}_{\mathbf{C}}\right)
$$

Pode-se definir a tensão efetiva $\bar{\sigma}$ como a tensão aplicada à parte resistente da seção. Assim, considerando-se as equações (6.67) e (6.69), tem-se:

$$
\bar{\sigma}=\frac{\mathbf{F}}{\overline{\mathbf{S}}}=\frac{\sigma \mathbf{S}}{\mathbf{S}\left(1-\mathbf{D}_{\mathrm{C}}\right)}=\frac{\sigma}{\left(1-\mathbf{D}_{\mathrm{C}}\right)}
$$

onde: $\bar{\sigma} \geq \sigma$,

$\bar{\sigma}=\sigma \quad$ representa um material íntegro,

$\bar{\sigma} \rightarrow \infty$ representa um estado limite de deteriorização.

A hipótese de deformação equivalente dada por LEMAITRE, CHABOCHE (1985) é a seguinte: "O mesmo estado de deformações de um material com dano pode ser derivado do material íntegro onde a tensão usual é substituída pela tensão efetiva”. Pode-se ilustrar essa hipótese da seguinte maneira:

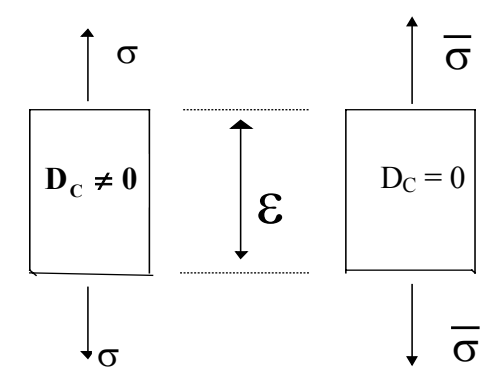

FIGURA 6.12 - Deformação Equivalente

Portanto, a deformação no material íntegro, que é equivalente à do material com dano, é dada por :

$$
\varepsilon=\frac{\bar{\sigma}}{\mathbf{E}}
$$


onde $\mathbf{E}$ é o módulo de elasticidade do material íntegro.

Logo, a deformação no material com dano é dada por:

$$
\varepsilon=\frac{\bar{\sigma}}{\mathbf{E}}=\frac{\sigma}{\mathbf{E}\left(1-\mathbf{D}_{\mathbf{C}}\right)}=\frac{\sigma}{\overline{\mathbf{E}}}
$$

onde $\overline{\mathbf{E}}$ é o módulo de elasticidade do material com dano.

\subsubsection{Modelo de Mazars}

Esse modelo apresenta bons resultados na análise de dano no concreto sujeito à carga proporcional ou cíclica. A variável de dano é função da deformação equivalente, que caracteriza o estado local de alongamento do material.

\section{* Hipóteses Básicas}

- O concreto em processo de dano evolutivo tem comportamento elástico, isto é, não há evolução de deformação plásticas num descarregamento, como é observado experimentalmente.

- Supõe-se que o dano seja causado somente pela existência de extensões (alongamentos), que ocorrerá ao longo de uma, ou mais, direções principais de deformação.

- Em geral, o dano conduz a uma anisotropia do concreto, que é considerado inicialmente como isótropo, mas a fim de simplificar o modelo, o dano será considerado como isótropo.

- O dano é representado por uma variável escalar $\mathbf{D}_{\mathbf{C}}$, cuja evolução ocorre quando a deformação equivalente $\bar{\varepsilon}$ ultrapassar um limite elástico dado.

\section{* Relações Elásticas entre Tensão e Deformação}

Pela lei de Hooke, o estado de tensão elástico para um caso de estado plano de tensão, em uma iteração $\mathbf{n}$, é dado por: 


$$
\underset{\sim}{\sigma_{\mathrm{n}}^{\mathrm{e}}}=\frac{\mathbf{E}}{\left(1-v^{2}\right)}\left[\begin{array}{ccc}
1 & v & \mathbf{0} \\
v & 1 & \mathbf{0} \\
\mathbf{0} & 0 & \frac{1-v}{2}
\end{array}\right] \underset{\sim}{\varepsilon_{\mathrm{n}}}
$$

onde: E é o módulo de elasticidade inicial e $v$ é o coeficiente de Poisson.

Passando-se as tensões, dadas por (6.73), para o sistema principal, pode-se escrever a tensão em uma dada direção principal i como:

$$
\begin{gathered}
\sigma_{i}=\sigma_{i}^{+}+\sigma_{i}^{-} \\
\text {onde: }\left\{\begin{array}{llll}
\text { se } & \sigma_{i}>\mathbf{0} & \sigma_{i}^{+}=\sigma_{i} & \sigma_{i}^{-}=\mathbf{0} \\
\text { se } & \sigma_{i}<0 & \sigma_{i}^{-}=\sigma_{i} & \sigma_{i}^{+}=\mathbf{0}
\end{array}\right.
\end{gathered}
$$

Assim, pela lei de Hooke, pode-se obter as deformações $\varepsilon_{T_{i}}$ devido às tensões de tração e as deformações $\varepsilon_{C_{i}}$ devido às tensões de compressão, em cada direção principal:

$$
\begin{aligned}
& \varepsilon_{T_{i}}=\frac{1+v}{\mathbf{E}} \sigma_{i}^{+}-\frac{v}{E} \sum_{j=1}^{3} \sigma_{j}^{+} \\
& \varepsilon_{C_{i}}=\frac{1+v}{E} \sigma_{i}^{-}-\frac{v}{E} \sum_{j=1}^{3} \sigma_{j}^{-}
\end{aligned}
$$

A deformação total $\varepsilon_{\mathrm{i}}$, em uma direção principal $\mathbf{i}$, é dada por:

$$
\varepsilon_{\mathrm{i}}=\varepsilon_{\mathrm{T}_{\mathrm{i}}}+\varepsilon_{\mathrm{C}_{\mathrm{i}}}
$$

\section{* Definição de Deformação Equivalente $\bar{\varepsilon}$}

A partir das deformações principais, dadas por (6.78), pode-se obter os alongamentos $\varepsilon_{\mathbf{i}}^{+} \quad$ nas direções principais i: 


$$
\begin{gathered}
\varepsilon_{i}^{+}=\frac{1}{2}\left(\varepsilon_{i}+\left|\varepsilon_{i}\right|\right) \\
\text { ou seja: }\left\{\begin{array}{lll}
\varepsilon_{i}^{+}=\varepsilon_{i} & \text { se } & \varepsilon_{i}>\mathbf{0} \\
\varepsilon_{i}^{+}=\mathbf{0} & \text { se } & \varepsilon_{i} \leq \mathbf{0}
\end{array}\right.
\end{gathered}
$$

Com o valor dos alongamentos $\varepsilon_{i}^{+}$, obtém-se a deformação equivalente $\bar{\varepsilon}$, que representa o estado de alongamento no sistema principal:

$$
\bar{\varepsilon}=\sqrt{\varepsilon_{1}^{+^{2}}+\varepsilon_{2}^{+^{2}}+\varepsilon_{3}^{+^{2}}}
$$

Deve-se observar que, segundo as hipótese feitas nesse trabalho, tem-se $\varepsilon_{3}^{+}=\mathbf{0}$.

\section{- Critério de Dano}

O dano aparece quando $\bar{\varepsilon}=\varepsilon_{\mathrm{d}_{0}}$, que é a deformação correspondente ao esforço máximo em uma prova de tração uniaxial, como é mostrado na figura abaixo:

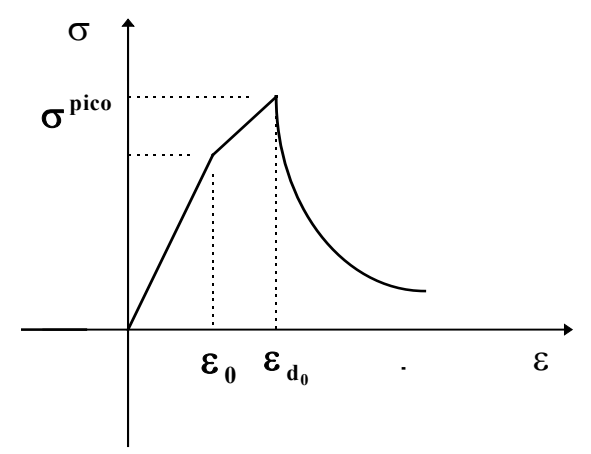

FIGURA 6.13 - Prova de Tração Uniaxial

Assim, o critério de dano é dado por:

$$
\mathbf{f}\left(\bar{\varepsilon}, \mathbf{D}_{\mathbf{C}}\right)=\bar{\varepsilon}-\mathbf{S}\left(\mathbf{D}_{\mathbf{C}}\right) \leq \mathbf{0}
$$


onde: $\mathbf{S}(\mathbf{0})=\varepsilon_{\mathbf{d}_{0}}$, é o limite elástico inicial e $\mathbf{S}\left(\mathbf{D}_{\mathbf{C}}\right)$ é igual à deformação equivalente da iteração anterior, se o ponto já estiver danificado, pois caso contrário, $\mathbf{S}\left(\mathbf{D}_{\mathrm{C}}\right)=\mathbf{S}(\mathbf{0})$.

Analogamente ao caso elasto-plástico, a evolução do dano $\mathbf{D}_{\mathbf{C}}$ (LEMAITRE e CHABOCHE, 1985), é dada por:

$$
\begin{aligned}
& \dot{\mathbf{D}}_{\mathrm{C}}=\mathbf{0} \text { se } \mathbf{f}\left(\bar{\varepsilon}, \mathbf{D}_{\mathbf{C}}\right) \leq \mathbf{0} \text { e } \dot{\mathbf{f}}\left(\bar{\varepsilon}, \mathbf{D}_{\mathrm{C}}\right)<\mathbf{0} \text { (caso de descarregamento) } \\
& \dot{\mathbf{D}}_{\mathbf{C}}>\mathbf{0} \text { se } \mathbf{f}\left(\bar{\varepsilon}, \mathbf{D}_{\mathrm{C}}\right)=\mathbf{0} \text { e } \dot{\mathbf{f}}\left(\bar{\varepsilon}, \mathbf{D}_{\mathrm{C}}\right)=\mathbf{0} \text { (caso de carregamento) }
\end{aligned}
$$

No caso bidimensional, pode-se ter também o caso de carregamento neutro $\left(\dot{\mathbf{f}}\left(\bar{\varepsilon}, \mathbf{D}_{\mathbf{C}}\right)=\mathbf{0}\right)$, onde não há evolução do dano $\left(\dot{\mathbf{D}}_{\mathrm{C}}=\mathbf{0}\right)$.

Não se admite $\mathbf{f}>\mathbf{0}$; quando isso ocorre, há evolução da variável de dano e o novo limite elástico passa a ser $\mathbf{S}\left(\mathbf{D}_{\mathbf{C}}\right)$, que é dado pelo estado de alongamento atual:

$$
\mathbf{S}\left(\mathbf{D}_{\mathrm{C}}\right)=\sqrt{\varepsilon_{1}^{+^{2}}+\varepsilon_{2}^{+^{2}}+\varepsilon_{3}^{+^{2}}}
$$

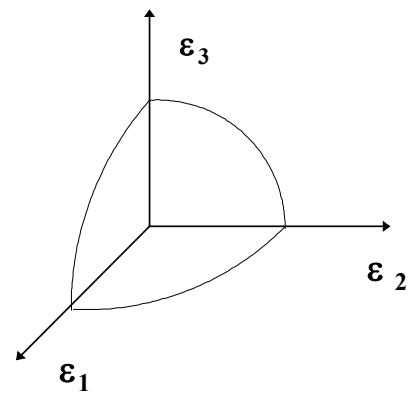

FIGURA 6.14 - Superfície de Ruptura em Deformação

A representação geométrica do critério no espaço, ou seja, a superfície de ruptura, é dada por um quarto de uma esfera de raio $\mathbf{S}\left(\mathbf{D}_{\mathbf{C}}\right)$, como é mostrado na figura (6.14). Se o ponto cair dentro da superfície, quer dizer que o estado de alongamento atual $\bar{\varepsilon}$ é menor que o limite elástico $\mathbf{S}\left(\mathbf{D}_{\mathbf{C}}\right)$ e, portanto, não há evolução do dano. Por outro lado, se o estado de alongamento atual $\bar{\varepsilon}$ for maior que $\mathbf{S}\left(\mathbf{D}_{\mathbf{C}}\right)$, o ponto cai fora da superfície e, portanto, há evolução do dano.

Para o caso bidimensional, a superfície de ruptura no espaço das tensões principais é dada por: 


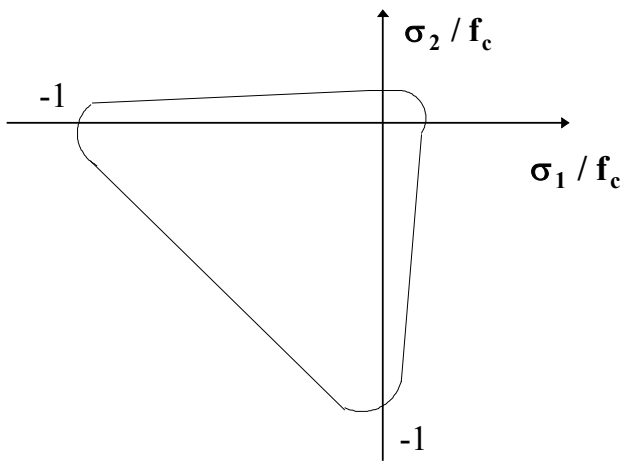

FIGURA 6.15 - Superfície de Ruptura em Tensão

onde $\mathbf{f}_{\mathbf{c}}$ é a resistência à compressão.

\section{* Cálculo da Variável de Dano}

Em tração uniaxial as microfissuras se desenvolvem perpendicularmente à direção do esforço e em compressão uniaxial paralelamente, como é mostrado na figura (6.16). Portanto, para descrever esse comportamento não simétrico do concreto, deve-se definir duas variáveis escalares de dano, uma para compressão $\mathbf{D}_{\mathbf{C C}}$ e outra para tração $\mathbf{D}_{\mathbf{C T}}$, cujas evoluções são governadas por leis independentes.
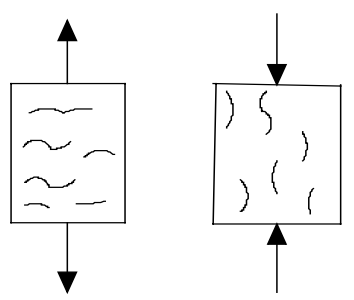

FIGURA 6.16 - Microfissuração em Tração e Compressão Uniaxiais.

No caso multiaxial, cada uma das componentes de tensão pode contribuir para a evolução do dano, assim a variável de dano é uma combinação linear de $\mathbf{D}_{\mathbf{C T}}$ e $\mathbf{D}_{\mathbf{C C}}$, as quais são definidas, de modo a representar com boa precisão as curvas experimentais de tração e compressão uniaxial para o caso de carregamento proporcional.

$$
\mathbf{D}_{\mathrm{C}}=\alpha_{\mathrm{T}} \mathbf{D}_{\mathrm{CT}}+\alpha_{\mathbf{C}} \mathbf{D}_{\mathrm{CC}}
$$


onde: $\quad 0 \leq \alpha_{\mathrm{C}} \leq \mathbf{1}, \mathbf{0} \leq \alpha_{\mathrm{T}} \leq 1$ e $\alpha_{\mathrm{T}}+\alpha_{\mathrm{C}}=\mathbf{1}$,

$$
\begin{aligned}
\mathbf{D}_{\mathrm{CT}} & =1-\frac{\varepsilon_{\mathrm{d}_{0}}\left(1-\mathbf{A}_{\mathrm{T}}\right)}{\bar{\varepsilon}}-\frac{\mathbf{A}_{\mathrm{T}}}{\exp \left[\mathbf{B}_{\mathrm{T}}\left(\bar{\varepsilon}-\varepsilon_{\mathrm{d}_{0}}\right)\right]} \\
\mathbf{D}_{\mathrm{CC}} & =1-\frac{\varepsilon_{\mathrm{d}_{0}}\left(1-\mathbf{A}_{\mathrm{C}}\right)}{\bar{\varepsilon}}-\frac{\mathbf{A}_{\mathrm{C}}}{\exp \left[\mathbf{B}_{\mathrm{C}}\left(\bar{\varepsilon}-\varepsilon_{\mathrm{d}_{0}}\right)\right]}, \\
\alpha_{\mathrm{T}} & =\frac{\sum_{\mathrm{i}=1}^{3} \varepsilon_{\mathrm{T}_{\mathrm{i}}}^{+}}{\varepsilon_{\mathrm{V}}^{+}} \\
\alpha_{\mathrm{C}} & =\frac{\sum_{\mathrm{i}=1}^{3} \varepsilon_{\mathrm{C}_{\mathrm{i}}}^{+}}{\varepsilon_{\mathrm{V}}^{+}} \\
\varepsilon_{\mathrm{V}}^{+} & =\sum_{\mathrm{i}=1}^{3} \varepsilon_{\mathrm{T}_{\mathrm{i}}}^{+}+\varepsilon_{\mathrm{C}_{\mathrm{i}}}^{+}
\end{aligned}
$$

onde $\varepsilon_{\mathrm{T}}$ e $\varepsilon_{\mathrm{C}}$ são obtidos, respectivamente, a partir das equações (6.76) e (6.77) e $\varepsilon_{\mathrm{T}}^{+}$e $\varepsilon_{\mathrm{C}}^{+}$ são obtidos considerando a equação (6.79).

- No caso de tração pura ter-se-á: $\alpha_{\mathbf{T}}=\mathbf{1}, \alpha_{\mathbf{C}}=\mathbf{0}$ e portanto: $\mathbf{D}_{\mathbf{C}}=\mathbf{D}_{\mathbf{C T}}$.

- Para compressão pura ter-se-á: $\alpha_{\mathrm{C}}=\mathbf{1}, \boldsymbol{\alpha}_{\mathrm{T}}=\mathbf{0}$ e portanto: $\mathbf{D}_{\mathrm{C}}=\mathbf{D}_{\mathbf{C C}}$.

No trabalho de ÁLVARES (1993), é descrito o processo de identificação dos parâmetros $\mathbf{E}, \mathbf{A}_{\mathrm{T}}, \mathbf{A}_{\mathbf{C}}, \mathbf{B}_{\mathrm{T}}, \mathbf{B}_{\mathbf{C}}$ e $\boldsymbol{\varepsilon}_{\mathbf{d}_{0}}$, que são característicos de cada material. Assim, utilizando-se o ensaio de compressão diametral para a identificação de $\boldsymbol{\varepsilon}_{\mathbf{d}_{0}}$, o ensaio de compressão uniaxial para a determinação de $\mathbf{E}, \mathbf{A}_{\mathbf{C}} \mathbf{B}_{\mathbf{C}}$ e um processo analítico de minimização do erro entre a resposta numérica e a experimental para a identificação de $\mathbf{A}_{\mathbf{T}}$, $\mathbf{B}_{\mathrm{T}}$, chegou-se aos seguintes valores médios:

$$
\mathrm{E}=29200 \mathrm{Mpa}, \mathrm{A}_{\mathrm{T}}=0.995, \mathrm{~B}_{\mathrm{T}}=8000, \varepsilon_{\mathrm{d}_{0}}=0.00007, \mathrm{~A}_{\mathrm{C}}=0.85, \mathrm{~B}_{\mathrm{C}}=1620 .
$$


Assim, a partir do estado de tensão elástico, dado pela equação (6.73), calcula-se a deformação equivalente $\bar{\varepsilon}$, através da equação (6.80), e verifica-se o critério. Se $\mathbf{f}>\mathbf{0}$, devese atualizar a variável de dano $\mathbf{D}_{\mathbf{C}}$, através da equação (6.84).

\section{* Estado de Tensão Verdadeiro}

- Considerando-se uma iteração $\mathbf{n}$ de um determinado incremento, através do incremento de curvaturas, calcula-se o incremento de deformações elásticas $\Delta \varepsilon_{\mathbf{n}}^{\mathbf{e}}$ pela equação (2.4). Soma-se esse último ao vetor de deformações da iteração anterior, obtendo-se o vetor de deformações totais $\varepsilon_{\mathbf{n}}$. Então, calcula-se as tensões supostas elásticas $\sigma_{\mathbf{n}}^{\mathbf{e}}$ através da equação (6.73).

- Passa-se $\sigma_{\mathbf{n}}^{\mathrm{e}}$ para o sistema principal e através das equações (6.76), (6.77), (6.78) e (6.79) calcula-se os alongamentos $\varepsilon_{\mathrm{i}}^{+}$nas direções principais. Então, calcula-se o alongamento equivalente $\bar{\varepsilon}_{\mathbf{n}}$ através da equação (6.80).

- Verifica-se o critério:

$\Rightarrow$ Se $\bar{\varepsilon}_{\mathbf{n}} \leq \varepsilon_{\mathbf{d}_{0}}$ : o ponto não está danificado: $\mathbf{D}_{\mathbf{C}(\mathbf{n})}=\mathbf{0}$.

$\Rightarrow$ Se $\bar{\varepsilon}_{\mathbf{n}}>\varepsilon_{\mathbf{d}_{0}}$ : o ponto está danificado. Nesse caso, deve-se comparar $\bar{\varepsilon}_{\mathbf{n}}$ com a deformação equivalente da iteração anterior $\mathbf{S}\left(\mathbf{D}_{\mathrm{C}}\right)=\bar{\varepsilon}_{\mathrm{n}-1}$ :

- Se $\mathbf{f}=\bar{\varepsilon}_{\mathrm{n}}-\bar{\varepsilon}_{\mathrm{n}-1} \leq \mathbf{0}$, tem-se um descarregamento e portanto, o incremento é elástico. Não precisa atualizar a variável de dano, ou seja: $\mathbf{D}_{\mathbf{C ( n )}}=\mathbf{D}_{\mathbf{C ( n - 1 )}}$.

- Se $\mathbf{f}>\mathbf{0}$, tem-se um carregamento. Deve-se atualizar a variável de dano, através da equação (6.84).

- O estado de tensão verdadeiro $\sigma_{\mathbf{n}}^{\mathrm{v}}$ é dado por:

$$
\underset{\sim}{\sigma_{\mathrm{n}}^{\mathrm{v}}}=\left(1-\mathbf{D}_{\mathrm{C}(\mathrm{n})}\right) \underset{\sim}{\mathbf{D}_{0}} \varepsilon_{\sim}
$$

onde $\mathbf{D}_{\mathbf{0}}$ é o tensor elástico do material íntegro. Para o caso de estado plano de tensão, temse: 
$\mathbf{D}_{0}=\frac{\mathbf{E}}{\left(1-v^{2}\right)}\left[\begin{array}{ccc}1 & v & 0 \\ v & 1 & 0 \\ \mathbf{0} & 0 & \frac{1-v}{2}\end{array}\right]$

Pode-se representar graficamente o modelo da seguinte forma:
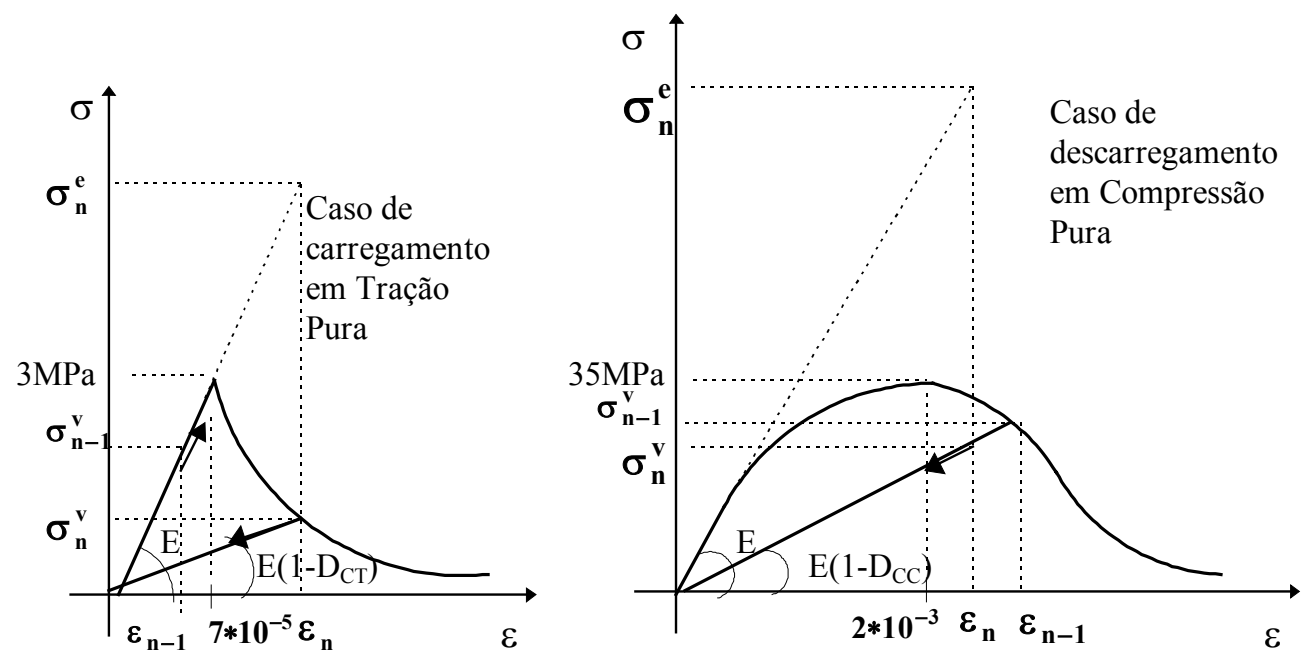

FIGURA 6.17 - Modelo de MAZARS em Tração e Compressão

Através desse algoritmo, pode-se observar que se o ponto estiver danificado numa iteração (n-1) e na iteração seguinte o mesmo sofrer descarregamento de tal forma que sua deformação equivalente $\bar{\varepsilon}_{\mathbf{n}}$ seja menor que $\varepsilon_{\mathbf{d}_{0}}$, o ponto recupera sua rigidez inicial E. Essa recuperação de rigidez não está representada na figura (6.17), porém ela é considerada, porque no processo iterativo utilizado para encontrar a posição correta da linha neutra (ver item 7.3), uma das estimativas da posição da linha neutra é feita considerando-se a seção toda comprimida. Desse modo, pontos que estavam muito danificados em tração, são submetidos à tensões de compressão e, nesse caso, como é observado experimentalmente, há recuperação da rigidez inicial. 


\section{SOLUÇÃO NÃO-LINEAR DE PLACAS SUJEITAS A CARGAS TRANSVERSAIS}

\subsection{Introdução}

A solução não-linear de uma placa sujeita a cargas transversais, é obtida através da formulação de placas apresentada no capítulo (5), que considera um campo de momentos iniciais. A solução numérica apresentada é baseada no processo de tensões iniciais, descrita no trabalho de ZIENKIEWICS et al. (1969), bastante empregada com o Método dos Elementos Finitos (MEF). Nesse trabalho, será considerado somente a não-linearidade física, aqui representada por modelos elasto-plásticos e da mecânica do dano.

A solução de um problema não-linear é incremental-iterativa e depende da 'história' do carregamento. É incremental, pois o cálculo é dividido em vários incrementos de carga, para permitir a aproximação linearizada do fenômeno. É iterativa, porque em um incremento de carga, a solução não-linear é obtida após $\mathbf{n}$ iterações, quando o equilíbrio da estrutura tenha sido verificado, com um erro aceitável. Se o processo não convergir dentro de um limite máximo de iterações prestabelecido, considera-se que a estrutura não é mais capaz de encontrar um estado de equilíbrio, isto é, a carga limite foi ultrapassada. No trabalho de OWEN\& HINTON (1980) está detalhada a abordagem numérica que foi empregada no presente trabalho. 


\subsection{Modelo Estratificado}

Admite-se que a placa é dividida em camadas, as quais podem ter espessuras e propriedades diferentes, considerando-se, porém, constantes as propriedades sobre cada camada, como é mostrado no trabalho de FIGUEIRAS (1983). O cálculo em camadas é importante numa análise não-linear, pois permite representar a distribuição não-linear das tensões ao longo da espessura e é essencial na análise de placas compostas de materiais diferentes, como é o caso da placa em concreto armado. Material e modelos constituivos distintos podem ser admitidos para cada camada. Deve-se notar, entretanto, que a distribuição da deformação ao longo da espessura é linear, mesmo se o estado de tensão já tenha atingido o comportamento não-linear.

\subsubsection{Cálculo do Momento Interno Resultante em uma Seção da Placa}

Para cada camada, atribui-se um valor de tensão associado a sua superfície média e considera-se que as componentes de tensão são constantes ao longo da espessura $\mathbf{t}_{\mathbf{n}} \mathrm{da}$ camada (ver figura 7.1). Assim, a distribuição das tensões ao longo da espessura poderia ser representada por retângulos, como está mostrado na figura (7.1). Nesse trabalho, porém, a distribuição será representada por polinômios. Não há necessidade de se definir a forma da distribuição das tensões nas camadas ou na espessura da placa, pois como a integração das tensões ao longo da espessura da placa de concreto, é feita através da fórmula de quadratura de Gauss, as propriedades do material e o valor das tensões precisam ser especificados apenas nos pontos de Gauss.

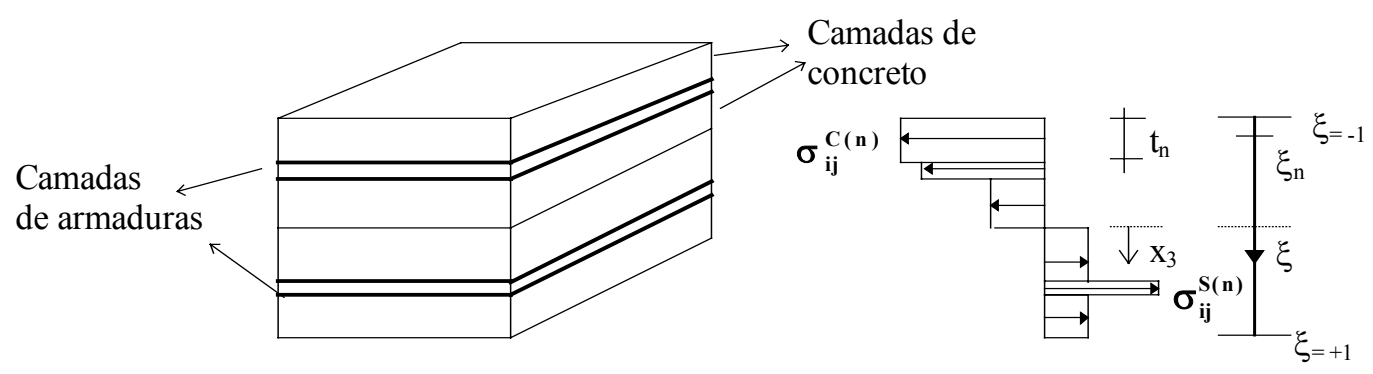

FIGURA 7.1 - Modelo Estratificado para o Concreto Armado

onde: $\quad-\mathbf{t} / \mathbf{2} \leq \mathbf{x}_{3} \leq \mathbf{t} / \mathbf{2}$, sendo $\mathbf{t}$ a espessura da placa, 


$$
\begin{aligned}
& x_{3}=\xi \frac{t}{2} \\
& d x_{3}=\frac{t}{2} d \xi
\end{aligned}
$$

Os pontos de Gauss, definidos ao longo da espessura em função da coordenada homogênea $\xi$, representarão as camadas de concreto, como é mostrado na figura (7.1), e as armaduras serão distribuídas em pontos adicionais, cujas posições são previamente estabelecidas. A espessura equivalente de uma camada de aço é tal que a área correspondente da armadura permaneça inalterada. Os valores das deformações seguem as regras de Bernoullie, enquanto as tensões devem obedecer o modelo constitutivo adotado.

A partir dessa aproximação, os momentos resultantes na seção são obtidos pela integração das respectivas componentes de tensão ao longo da espessura, ou seja:

$$
\mathbf{M}_{\mathrm{ij}}=\int_{-\mathbf{t} / \mathbf{2}}^{\mathbf{t} / \mathbf{2}} \sigma_{\mathrm{ij}}^{\mathbf{C}} \mathbf{x}_{3} \mathbf{d} \mathbf{x _ { 3 }}+\sum_{\mathbf{n}=\mathbf{1}}^{\mathrm{Ns}} \sigma_{\mathrm{ij}}^{\mathbf{S}(\mathbf{n})} \delta_{\mathrm{ij}} \mathbf{A}_{\mathbf{S}(\mathbf{n})} \mathbf{x}_{\mathbf{3 S}}^{\mathbf{n}} \quad(\mathrm{i}, \mathrm{j}=1,2)
$$

onde: $\quad \sigma_{\mathrm{ij}}^{\mathrm{C}}$ é a tensão na placa de concreto,

Ns é o número de armaduras,

$\mathbf{x}_{3 \mathbf{S}}^{\mathbf{n}}$ é a posição da armadura $\mathbf{n}$ considerada,

$\sigma_{\mathrm{ij}}^{\mathrm{S}(\mathbf{n})}$ é a tensão na armadura $\mathbf{n}$,

$\mathbf{A}_{\mathbf{S ( n )}}$ é a área da armadura $\mathbf{n}$.

Para a integração numérica do primeiro termo de (7.2), é necessário um número adequado de pontos de Gauss para que se obtenha uma boa aproximação das tensões e esforços resultantes satisfatórios.

Fazendo-se a mudança de coordenadas, indicada nas equações (7.1) e a integração numérica (item 4.7.1), a primeira parcela da equação(7.2), relativa à placa de concreto, pode ser escrita como:

$$
M_{i j}^{C}=\int_{-t / 2}^{t / 2} \sigma_{i j}^{C} x_{3} d x_{3}=\frac{t^{2}}{4} \int_{-1}^{1} \sigma_{i j}^{C} \xi d \xi=\frac{t^{2}}{4} \sum_{I G=1}^{N g} \sigma_{i j}^{C(I G)} \xi_{I G} W_{I G}
$$


onde Ng é o número de pontos de Gauss.

Logo, os momentos internos resultantes, em uma determinada seção da placa, são dados por:

$$
\mathbf{M}_{\mathrm{ij}}=\frac{\mathbf{t}^{2}}{4} \sum_{\mathrm{IG}=1}^{\mathrm{Ng}} \sigma_{\mathrm{ij}}^{\mathrm{C}(\mathrm{IG})} \xi_{\mathrm{IG}} \mathbf{W}_{\mathrm{IG}}+\sum_{\mathrm{n}=1}^{\mathrm{Ns}} \sigma_{\mathrm{ij}}^{\mathrm{s}(\mathbf{n})} \delta_{\mathrm{ij}} \mathbf{A}_{\mathbf{S}(\mathbf{n})} \mathbf{x}_{3 \mathrm{~S}}^{\mathbf{n}} \quad(\mathrm{i}, \mathrm{j}=1,2)
$$

\subsubsection{Processo para Zerar a Normal Resultante numa Seção da Placa}

O valor do momento interno resultante, dado pela equação (7.4), é referente a uma placa composta de somente um material, que tenha comportamento simétrico em tração e compressão. Nesse caso, o momento interno é calculado considerando-se que a linha neutra, na seção considerada, passa pelo seu ponto médio $\left(\mathbf{x}_{3}=\mathbf{0}\right)$, isto é, admite-se que a distribuição de tensões é simétrica em tração e compressão. Contudo, deve-se levar em consideração, que o comportamento do concreto não é igual em tração e compressão e além disso, é usual que as armaduras não sejam simetricamente distribuídas. Com isso, a linha neutra não coincide mais com a superfície média da placa. Como no caso de flexão simples, que é o caso considerado nesse trabalho, a placa não pode ser submetida à forças normais, somente à cargas transversais, deve-se procurar a nova posição da linha neutra para que se tenha a força normal resultante nula, a fim de se continuar a ter um caso de flexão simples.

O comportamento não linear e/ou não simétrico dos materiais faz com que uma mudança na posição da linha neutra em uma determinada direção $\left(\mathbf{x}_{1}, \mathbf{x}_{2}\right.$ ou $\left.\mathbf{x}_{1} \mathbf{x}_{2}\right)$ influencie o valor da normal resultante em outra direção. Portanto, as posições da linha neutra nas três direções são dependentes e consequentemente, cada vez que se estima a posição da mesma em uma direção, deve-se verificar a força normal resultante nas outras direções. Assim, primeiro faz-se com que a normal resultante na direção $\mathbf{x}_{\mathbf{1}}$ seja nula, então estima-se a posição da linha neutra em $\mathbf{x}_{2}$ e verifica-se o valor da normal em $\mathbf{x}_{1}$. Esse processo continua até que a normal resultante nessas duas direções sejam nulas. Em seguida, estima-se a posição da linha neutra em $\mathbf{x}_{1} \mathbf{x}_{2}$ e verifica-se as direções $\mathbf{x}_{1}$ e $\mathbf{x}_{2}$. No final desse processo iterativo, ter-se-á a normal resultante nula nas três direções.

A estimativa da nova posição $\mathbf{Z}_{\mathbf{i j}}$ da linha neutra, em uma direção $\mathbf{i j}$, é feita por interpolação linear, como é mostrado na figura (7.2), usando-se os valores das normais $\mathbf{N}_{\mathrm{ij}}^{1} \mathrm{e}$ $\mathbf{N}_{\mathrm{ij}}^{2}$ previamente calculados, respectivamente, para as posições $\mathbf{Z}_{\mathrm{ij}}^{1}$ e $\mathbf{Z}_{\mathrm{ij}}^{2}$ da linha neutra. Assim, a nova posição $\mathbf{Z}_{\mathrm{ij}}$ é dada por: 


$$
Z_{i j}=Z_{i j}^{2}+\frac{N_{i j}^{2}\left(Z_{i j}^{1}-Z_{i j}^{2}\right)}{N_{i j}^{2}-N_{i j}^{1}}
$$

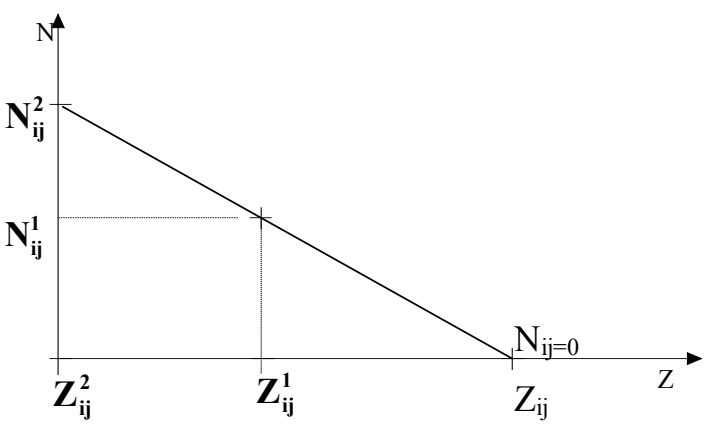

FIGURA 7.2 - Estimativa da Linha Neutra

Na primeira estimativa de $\mathbf{Z}_{\mathrm{ij}}$ não há um segundo ponto $\mathbf{Z}_{\mathrm{ij}}^{2}$ para se fazer a interpolação, então adota-se, o mesmo de tal forma que a seção fique toda comprimida. Assim, calcula-se o valor da normal para essa posição e então faz-se a primeira estimativa de $\mathbf{Z}_{\mathrm{ij} \text {., }}$ calculando-se a normal $\mathbf{N}_{\mathrm{ij}}$ para essa posição. A partir da segunda estimativa, ter-se-á três valores de normais: $\mathbf{N}_{\mathrm{ij}}^{\mathbf{1}}$ e $\mathbf{N}_{\mathrm{ij}}^{\mathbf{2}}$ da interpolação anterior e $\mathbf{N}_{\mathrm{ij}}$. Assim, para a interpolação seguinte, adota-se $\mathbf{N}_{\mathrm{ij}}^{2}=\mathbf{N}_{\mathrm{ij}}$ e $\mathbf{N}_{\mathrm{ij}}^{1}$ será igual a:

$$
\mathbf{N}_{\mathrm{ij}}^{1}=\mathbf{N}_{\mathrm{ij}}^{\prime^{2}} \text { se }\left|\mathbf{Z}_{\mathrm{ij}}^{\prime 2}-\mathbf{Z}_{\mathrm{ij}}\right|<\left|\mathbf{Z}_{\mathrm{ij}}^{\mathbf{1}^{1}}-\mathbf{Z}_{\mathrm{ij}}\right|
$$

ou

$$
\mathbf{N}_{\mathrm{ij}}^{1}=\mathbf{N}_{\mathrm{ij}}^{{ }^{1}} \text { se }\left|\mathbf{Z}^{\prime 1 \mathrm{j}}-\mathbf{Z}_{\mathrm{ij}}\right|<\left|\mathbf{Z}_{\mathrm{ij}}^{\mathbf{i j}^{2}}-\mathbf{Z}_{\mathrm{ij}}\right|
$$

A cada nova estimativa da linha neutra, deve-se calcular o incremento de deformações $\Delta \varepsilon_{\mathrm{ij}}^{\mathbf{L N}}$ e de tensões $\Delta \sigma_{\mathrm{ij}}^{\mathbf{L N}}$, devido à mudança da mesma. $\mathrm{O}$ incremento de deformações é dado por:

$$
\Delta \varepsilon_{\mathrm{ij}}^{\mathrm{LN}}=\left(\mathbf{Z}_{\mathrm{ij}}^{\mathbf{0}}-\mathbf{Z}_{\mathrm{ij}}\right) \mathbf{w},_{\mathrm{ij}} \quad(\mathrm{i}, \mathrm{j}=1,2)
$$


onde $\mathbf{Z}_{\mathrm{ij}}^{\mathbf{0}}$ é a posição da linha neutra no início da iteração e $\mathbf{w},_{\mathrm{ij}}$ é o vetor das curvaturas.

Somando-se $\Delta \varepsilon_{\mathrm{ij}}^{\mathrm{LN}}$ ao incremento de deformações elástico $\Delta \varepsilon_{\mathrm{ij}}^{\mathrm{e}}$ devido ao carregamento (equação 2.4), obtém-se o incremento de deformação total $\Delta \varepsilon_{\mathrm{ij}}^{\mathrm{T}}$. Somando-se esse último ao vetor de deformações verdadeiras da iteração anterior $\Delta \varepsilon_{\mathrm{ij}}^{\mathrm{n}-1}$, obtém-se o vetor de deformações totais $\varepsilon_{\mathbf{i j}}^{\mathbf{n}}$, como é mostrado na figura (7.3) para uma dada direção ij.

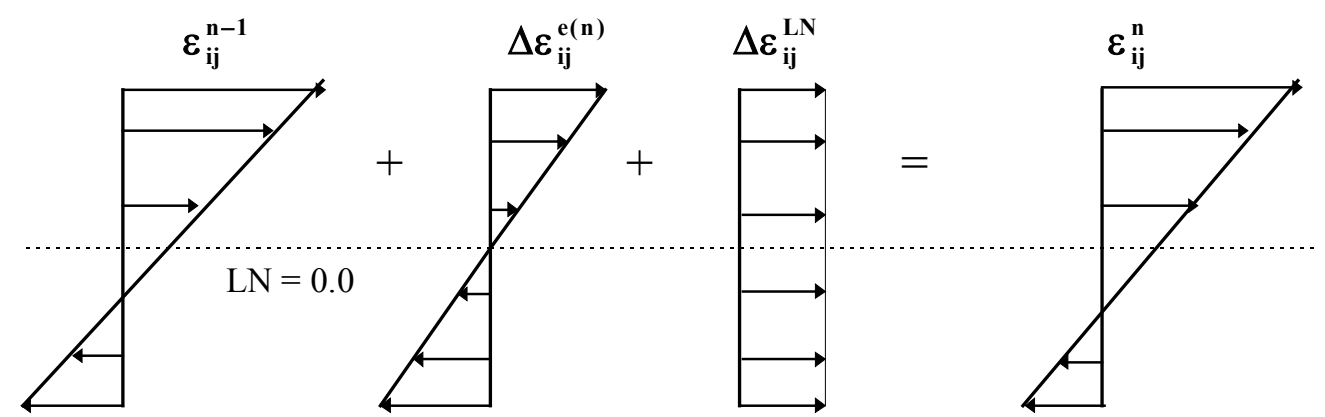

FIGURA 7.3 - Distribuição das Deformações em uma Seção da Placa

A fim de se determinar a posição da linha neutra $\mathbf{Z}_{\mathbf{i j}}^{\mathbf{0}}$, na direção $\mathbf{i j}$ e no início de uma iteração $\mathbf{n}$, considere a figura (7.4), onde $\mathbf{P}$ representa um ponto de Gauss qualquer ao longo da espessura. A posição do ponto $\mathbf{P}$ em relação à linha neutra atual é dada por:

$$
Z_{\mathrm{ij}}^{0(P)}=\frac{\varepsilon_{\mathrm{ij}}^{\mathrm{n}}}{\mathbf{w},{ }_{\mathrm{ij}}^{\mathbf{n}}}
$$

e a posição do ponto $\mathbf{P}$ em relação à linha neutra na posição $(0,0)$ é dada por:

$$
Z^{P}=\frac{\mathbf{t}}{2} \xi_{I G}
$$

Logo, de acordo com a figura (7.4), a posição inicial da linha neutra é dada por:

$$
\mathbf{Z}_{\mathrm{ij}}^{\mathbf{0}}=\mathbf{Z}^{\mathrm{p}}-\mathbf{Z}_{\mathrm{ij}}^{0(\mathbf{P})}
$$



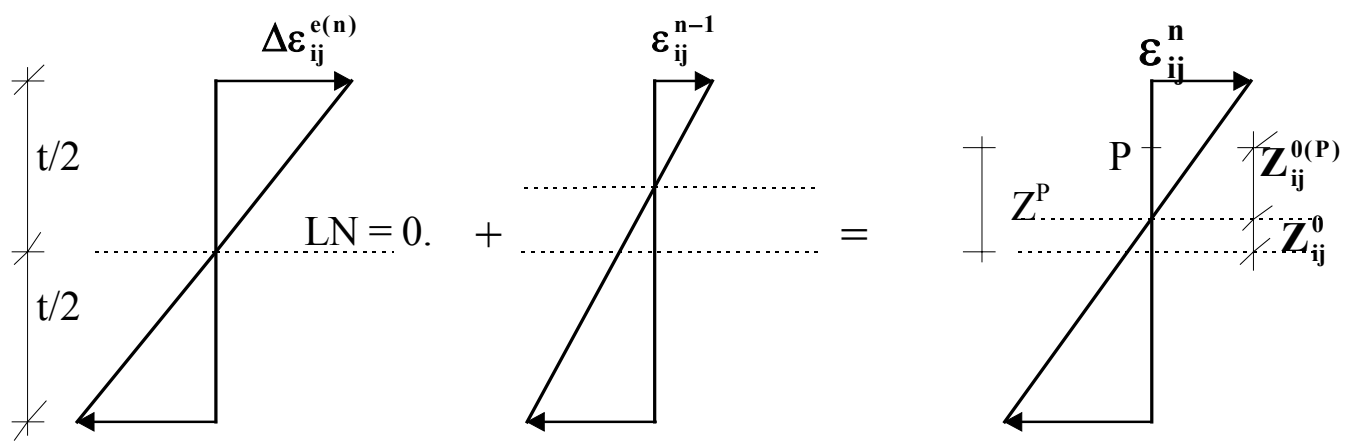

FIGURA 7.4 - Distribuição de Deformação Resultante em uma Seção

Com o incremento de deformações $\Delta \varepsilon_{\mathrm{ij}}^{\mathrm{LN}}$, calcula-se o incremento de tensões $\Delta \sigma_{\mathrm{ij}}^{\mathrm{LN}}$ na placa de concreto, através da equação (2.5). Então, no caso do modelo elasto-plástico, soma-se $\Delta \sigma_{\mathrm{ij}}^{\mathrm{LN}}$ aos incrementos elásticos de tensão devido ao carregamento $\Delta \sigma_{\mathrm{ij}}^{\mathrm{e}(\mathrm{n})}$ da iteração em consideração, obtendo-se o incremento de tensão total $\Delta \sigma_{\mathrm{ij}}^{\mathrm{T}}$. Somando-se esse último às tensões verdadeiras da iteração anterior $\sigma_{\mathrm{ij}}^{\mathrm{V}(\mathbf{n}-\mathbf{1})}$, obtém-se uma nova distribuição de tensão $\Delta \sigma_{\mathrm{ij}}^{\mathrm{n}}$ na seção, como é mostrado na figura (7.5). Do mesmo modo, procede-se com as armaduras, sendo que o incremento de tensão nas armaduras, é dado por:

$$
\Delta \sigma_{\mathrm{ij}}^{\mathrm{S}(\mathrm{LN})}=\mathbf{E}_{\mathrm{s}} \Delta \varepsilon_{\mathrm{ij}}^{\mathrm{LN}} \delta_{\mathrm{ij}}
$$

onde $\mathbf{E}_{\mathbf{S}}$ é o módulo de elasticidade do aço

No caso do modelo de dano, calcula-se um estado de tensão elástico $\sigma_{\mathrm{ij}(\mathrm{n})}^{\mathrm{e}}$, através do estado de deformação total $\varepsilon_{\mathrm{ij}}^{\mathbf{n}}$.

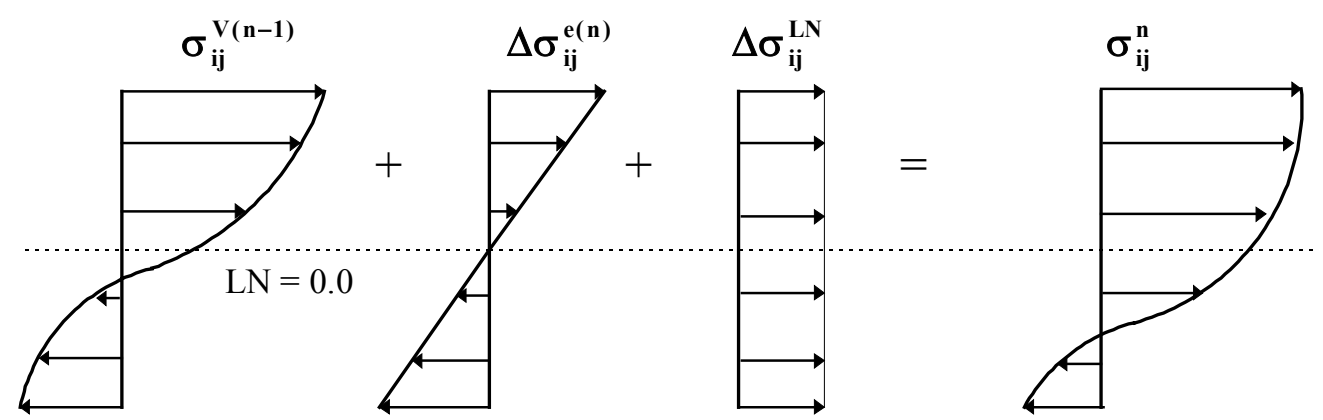

FIGURA 7.5 - Distribuição de Tensão Resultante em uma Seção 
Verifica-se, então, o modelo constitutivo para todos os pontos ao longo da espessura e calcula-se a nova normal resultante, que é dada por:

$$
\mathbf{N}_{\mathrm{CA}}=\int_{-t / 2}^{\mathrm{t} / 2} \sigma_{\mathrm{ij}}^{\mathrm{C}} \mathbf{d} \mathbf{x}_{3}+\sum_{\mathrm{n}=1}^{\mathrm{Ns}} \sigma_{\mathrm{ij}}^{\mathrm{S}(\mathrm{n})} \delta_{\mathrm{ij}} \mathbf{A}_{\mathrm{S}(\mathrm{n})}
$$

Fazendo-se a mudança de coordenadas, indicada em (7.1) e a integração numérica (item 4.7.1), obtém-se:

$$
\mathbf{N}_{\mathrm{CA}}=\frac{\mathbf{t}}{\mathbf{2}} \sum_{\mathrm{IG}=1}^{\mathrm{Ng}} \sigma_{\mathrm{ij}}^{\mathrm{C}(\mathrm{IG})} \mathbf{W}_{\mathrm{IG}}+\sum_{\mathrm{n}=1}^{\mathrm{Ns}} \sigma_{\mathrm{ij}}^{\mathrm{S}(\mathbf{n})} \delta_{\mathrm{ij}} \mathbf{A}_{\mathrm{S}(\mathbf{n})}
$$

Considera-se que a posição da linha neutra em uma dada direção está correta quando o valor absoluto da normal resultante nessa direção é menor que:

$$
\mathbf{N}_{\mathrm{CA}} \leq \text { tol. } \mathbf{f}_{\mathrm{ck}} \cdot \mathbf{t}
$$

onde $\mathbf{f}_{\mathbf{c k}}$ é a resistência à compressão, $\mathbf{t}$ a espessura da placa e tol a tolerância de cálculo.

\subsection{Processo Incremental}

Definido o carregamento total previsto, divide-se o mesmo em vários incrementos, definindo-se, para cada incremento $\mathbf{i}$, o coeficiente $\beta_{\mathbf{i}}$ de multiplicação do carregamento total. Assim, o novo incremento de carga pode ser definido de acordo com a perfomance do processo iterativo anterior, ou seja, se no incremento anterior o processo iterativo alcançou o limite máximo de iterações, diminui-se o incremento de carga, se não, aumenta-se o incremento de carga. Logo, em trechos de forte não-linearidade, quanto menores os incrementos de carga, melhor será aproximada a curva não linear. Para cada incremento define-se o número máximo de iterações, a tolerância de convergência e o coeficiente de multiplicação do carregamento desejados.

Passa-se ao incremento seguinte, se o critério de convergência do incremento anterior foi verificado, caso contrário, o cálculo é interrompido, mesmo se ainda houver outros incrementos definidos. 


\subsection{Processo Iterativo}

No caso de não-linearidade física, procura-se o equilíbrio em um dado incremento de carga, através do Método de Newton-Raphson, considerando-se o nível de carga ( $\left.\mathbf{M}_{\text {ext }}\right)$ constante, como é mostrado na figura (7.6). Nesse trabalho será considerado apenas o Método de Newton-Raphson Modificado, descrito a seguir na figura (7.7).

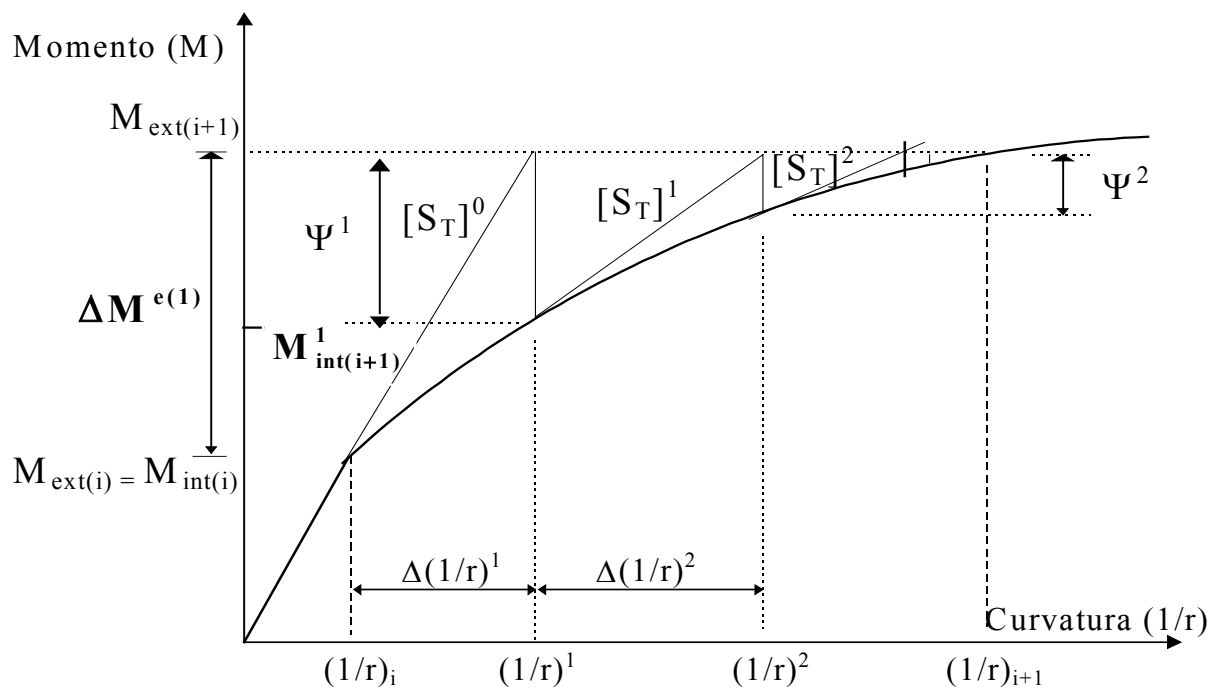

FIGURA 7.6 - Método de Newton Raphson Padrão

onde: $\left[\mathbf{S}_{\mathbf{T}}\right]^{\mathrm{n}}$ é a matriz tangente na iteração $\mathbf{n}$,

$(1 / r)^{n}$ é o vetor de curvaturas da iteração $n$,

$\Psi^{\mathrm{n}}$ é o vetor de resíduo da iteração $\mathbf{n}$,

$\mathbf{M}_{\text {ext(i+1) }}$ é o vetor de momentos externos do incremento i+1,

$\Delta \mathbf{M}^{\mathbf{e}(\mathbf{n})}$ é o incremento de momentos elásticos da iteração $\mathbf{n}$,

$\mathbf{M}_{\text {int(i+1) }}^{\mathbf{n}}$ é o vetor de momentos internos verdadeiros da iteração $\mathbf{n}$, do incremento $\mathbf{i}+1$,

$(\mathbf{1} / \mathbf{r})_{\mathrm{i}}$ é o vetor de curvaturas do incremento $\mathbf{i}$.

Na primeira iteração do incremento (figura 7.6), tem-se um incremento de momentos $\Delta \mathbf{M}^{\mathbf{e}(1)}$, que foi calculado elasticamente, considerando-se as relações de equilíbrio da estrutura. Se o mesmo não for totalmente elástico, deve-se fazer novas iterações, até que se encontre um estado de momentos internos aproximadamente igual ao estado de momentos 
externos. Deve-se observar, que no final da iteração, os incrementos de curvatura e de deformação são os mesmos que aqueles no começo da mesma. Entretanto, ocorrerá mudanças nos estados de momentos e de tensões, se a iteração não for elástica, isto é, se o incremento de momentos verdadeiros $\Delta \mathbf{M}_{\text {int }}^{\mathrm{n}}$, obtido no final da iteração, não for igual àquele aplicado inicialmente $\Delta \mathbf{M}^{\mathbf{e}(\mathbf{n})}$. A diferença entre os dois valores resultará no incremento de momentos residuais $\Psi^{\mathbf{n}}$ que será aplicado ao sistema de equações, como um campo de momentos iniciais, a fim de se obter o incremento de momentos elásticos a ser aplicado na próxima iteração. $\mathrm{O}$ incremento terá convergido quando esse incremento de momentos residuais se aproximar de zero, em todos os pontos da estrutura, o que é traduzido pelo critério de convergência utilizado.

A matriz [S ], que é dada pela equação (5.111) e representa a influência dos momentos iniciais nos momentos do contorno e do domínio da estrutura, é constante num incremento linear, mas num incremento não linear ela é igual à matriz tangente à curva incremental no tempo considerado, como é mostrado no Método de Newton-Raphson padrão (figura 7.6). Nesse caso, a convergência é muito rápida e portanto, são necessários poucas iterações.

Nesse trabalho será considerado somente o Método de Newton-Raphson Modificado, que considera a matriz $[\mathbf{S}]$ constante e igual àquela obtida na primeira iteração do primeiro incremento, como é mostrado na figura (7.7). O procedimento de cálculo considerando-se o Método de Newton-Raphson padrão está descrito no trabalho de CHAVES (1997).

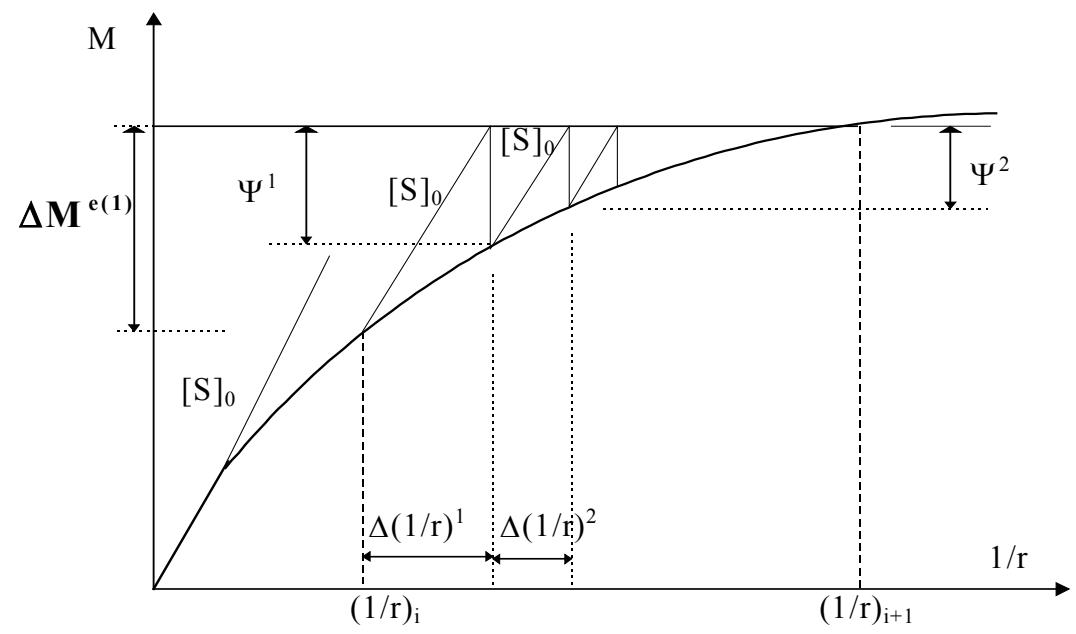

FIGURA 7.7 - Método de Newton Raphson Modificado

\subsubsection{Critério de Convergência}


O critério de convergência do processo iterativo é dado pela relação entre o módulo de incremento de tensão efetiva $\Delta \bar{\sigma}$, suposto elástico, e a tensão de escoamento $\sigma_{\mathbf{y}}^{\mathbf{n}}$ da iteração $\mathbf{n}$ em consideração, no caso de um modelo elasto-plástico, ou pela relação entre o módulo de incremento de alongamento equivalente $\Delta \bar{\varepsilon}$ e o limite elástico $\mathbf{S}\left(\mathbf{D}_{\mathbf{C}}\right)$ no caso do modelo de dano. Toma-se os valores em módulo devido ao fato de que mesmo numa iteração elástica, o momento interno pode não ser igual ao externo devido à presença de armaduras, as quais aumentam a rigidez da placa. Logo, em todos os pontos de Gauss e em todas as armaduras, referentes a uma seção da placa, deve-se ter:

$$
\frac{|\Delta \bar{\sigma}|}{\sigma_{\mathrm{y}}^{\mathrm{n}}} \leq \text { toler â ncia }
$$

ou no caso do modelo de dano:

$$
\frac{|\Delta \bar{\varepsilon}|}{\mathbf{S}\left(\mathbf{D}_{\mathrm{C}}\right)} \leq \text { toler â ncia }
$$

A tolerância no critério de convergência, deve ser tal que dê resultados coerentes, sem a necessidade de realizar muitas iterações. Assim, tolerâncias muito 'frouxas', produzem resultados não muito confiáveis, pois refletem falsos estados de equilíbrio; e tolerâncias muito 'apertadas' elevam o esforço computacional, fazendo iterações desnecessárias.

No caso de soluções divergentes, deve-se reduzir os tamanhos dos incrementos e investigar a possibilidade de colapso da estrutura. Para soluções com convergência lenta, deve-se reduzir o tamanho dos incrementos, aumentar a tolerância de convergência, ou usar o método Newton Raphson padrão, sendo que essa última opção não foi prevista no trabalho.

\subsection{Procedimento de Cálculo}

No início de cada iteração, tem-se um estado de tensão na estrutura, que é estaticamente admissível, pois verifica as condições de equilíbrio da estrutura. Deve-se, então verificar se em toda a estrutura o modelo constitutivo é obedecido. Assim, para uma iteração $\mathbf{n}$ de um incremento $\mathbf{i}$, tem-se um incremento de momentos elásticos $\left\{\Delta \mathbf{M}^{\mathrm{e}}\right\}$, para cada ponto do contorno e do domínio, que é determinado da seguinte maneira: 


$$
\begin{array}{ll}
\left\{\Delta \mathbf{M}^{\mathrm{e}}\right\}_{i}^{1}=\beta_{i}\{\mathbf{N}\} \quad \text { se } \quad \mathbf{n}=\mathbf{1} \\
\left\{\Delta \mathbf{M}^{\mathrm{e}}\right\}_{i}^{\mathrm{n}}=[\mathbf{S}]\left\{\Delta \mathbf{M}^{0}\right\}_{i}^{\mathrm{n}-1} \quad \text { se } \quad \mathbf{n} \geq \mathbf{2}
\end{array}
$$

sendo que $\{\mathbf{N}\}$ e $[\mathbf{S}]$ dados, respectivamente, por (5.110) e (5.111).

Assim, considerando-se um determinado ponto do contorno ou do domínio, deve-se proceder da seguinte maneira:

- Com $\left\{\Delta \mathbf{M}^{\mathrm{e}}\right\}_{\mathrm{i}}^{\mathrm{n}}$, através da relação elástica (2.10) calcula-se o incremento de curvaturas $\left\{\Delta(\mathbf{1} / \mathbf{r})^{\mathrm{e}}\right\}_{\mathrm{i}}^{\mathrm{n}}$ e, então, para cada ponto ao longo da espessura, determina-se o incremento de deformações $\left\{\Delta \varepsilon^{\mathrm{e}}\right\}_{\mathrm{i}}^{\mathrm{n}}$ devido ao carregamento, através da equação (2.4), obtendo-se então :

$$
\begin{aligned}
& \{1 / r\}_{i}^{n}=\{1 / r\}_{i}^{n-1}+\left\{\Delta(1 / r)^{e}\right\}_{i}^{n} \\
& \{\varepsilon\}_{i}^{n}=\{\varepsilon\}_{i}^{n-1}+\left\{\Delta(\varepsilon)^{e}\right\}_{i}^{n}
\end{aligned}
$$

- No caso do modelo elasto-plástico, calcula-se também o incremento de tensões elásticas $\left\{\Delta \sigma^{\mathrm{e}}\right\}_{\mathrm{i}}^{\mathrm{n}}$, devido ao carregamento, através da equação (2.7) se for um ponto de Gauss ou pela equação (7.12) se for uma armadura. Soma-se esse último ao estado de tensão verdadeiro da iteração anterior e tira-se a tração nos pontos de Gauss. Para o modelo de dano, calcula-se as tensões elásticas $\left\{\sigma^{\mathrm{e}}\right\}_{\mathrm{i}}^{\mathbf{n}}$ através da equação (6.73). Verifica-se o modelo constitutivo, obtendo-se o vetor de tensão verdadeiro $\left\{\sigma^{\mathbf{v}}\right\}_{i}^{n}$. e o incremento de tensão verdadeiro $\left\{\Delta \sigma^{v}\right\}_{i}^{n}$ para o ponto em questão. Procedendo-se, da mesma forma, para todos os pontos de Gauss e armaduras, obtém-se uma nova distribuição de tensão ao longo da espessura.

- Verifica-se, então, a normal resultante nas três direções. Se alguma não for nula, estimase a nova posição da linha neutra, verifica-se de novo os modelos constitutivos em todos os pontos e calcula-se a novas normais resultante. Esse processo continua até que as normais sejam nulas nas três direções. Desse modo, obtém-se a verdadeira distribuição de tensões ao longo da espessura. 
- Com a equação (7.4), obtêm-se o vetor de momentos verdadeiros $\left\{\mathbf{M}^{\mathbf{v}}\right\}_{\mathrm{i}}^{\mathbf{n}}$, e o vetor de incremento de momentos verdadeiros $\left\{\Delta \mathbf{M}^{\mathbf{v}}\right\}_{\mathrm{i}}^{\mathbf{n}}$.

- Calcula-se, então, o vetor de momentos residuais :

$$
\left\{\Delta \mathbf{M}^{0}\right\}_{i}^{n+1}=\{\Psi\}_{i}^{n}=\left\{\Delta \mathbf{M}^{\mathrm{e}}\right\}_{i}^{n}-\left\{\Delta \mathbf{M}^{v}\right\}_{i}^{n}
$$

- Para todos os pontos ao longo da espessura, verifica-se o critério de convergência, através da equação (7.16) se o modelo considerado for o elasto-plástico ou da equação (7.17) se for o modelo de dano.

Segue-se o mesmo procedimento para todos os pontos do contorno e do domínio. Se o critério de convergência não for verificado, para algum ponto, quer dizer que o estado de tensão na estrutura é tal que verifica o modelo constitutivo em todos os pontos, mas não é mais estaticamente admissível. Assim, aplica-se $\{\Psi\}_{i}^{n}$ ao sistema como um campo de momentos iniciais, obtém-se um novo incremento de momentos elásticos através da equação (7.18.b) e passa-se à iteração seguinte. Caso o critério de convergência seja verificado, passa-se ao incremento seguinte. Portanto, o processo iterativo termina quando o estado de tensão na estrutura verificar ao mesmo tempo, as condições de equilíbrio e o modelo constituivo em todos os pontos da mesma.

Ao final de um incremento, têm-se:

$$
\begin{aligned}
& \underset{\sim}{\mathbf{X}}=\underset{\sim}{\mathbf{L}}+\underset{\sim}{\mathbf{R}}{\underset{\sim}{\mathbf{M}}}^{\mathbf{0}} \\
& \underset{\sim}{\mathbf{W}(\mathbf{q})}={\underset{\sim}{\mathbf{L}}}^{\mathbf{L}}+\underset{\sim}{\mathbf{R}^{*}}{\underset{\sim}{\mathbf{M}^{\mathbf{0}}}}^{\mathbf{M}}=\mathbf{M}^{\mathbf{v}}
\end{aligned}
$$

onde: $\quad \mathbf{M}^{0}$ é vetor de momentos plásticos acumulados, ou vetor dos resíduos acumulados, os outros vetores e matrizes são os mesmos definidos em (5.100) e (5.104).

\subsection{Exemplos Numéricos}


A seguir serão apresentados um exemplo de viga e dois exemplos de placas, sendo que os resultados desses últimos serão comparados com os resultados obtidos experimentalmente. Apesar de todos os exemplos apresentarem eixos de simetria, será considerado sempre a placa inteira, para evitar de se prescrever valores no domínio da mesma.

\subsubsection{Viga apoiada com momento aplicado nas extremidades}

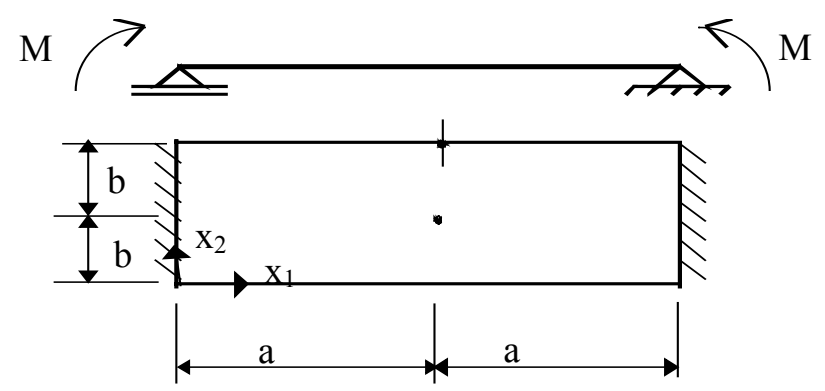

FIGURA 7.8 - Viga Simplesmente Apoiada

Na viga representada na figura (7.8), os lados de dimensão 2b são apoiados e os 
outros dois são livres. O momento $\mathbf{M}$, aplicado ao longo dos lados apoiados, é igual a $50000 \mathrm{Kgfxcm}$. A geometria da placa é definida por: $\mathrm{a}=75 \mathrm{~cm}, \mathrm{~b}=10 \mathrm{~cm}$ e a espessura $\mathrm{t}=30 \mathrm{~cm}$. Para a placa de concreto, utilizou-se um módulo de elasticidade $\mathrm{E}_{\mathrm{C}}=300000$ $\mathrm{kgf} / \mathrm{cm}^{2}$, coeficiente de poisson $v$ igual a 0 , tensão de plastificação $\mathrm{f}_{\mathrm{C}}=300 \mathrm{Kgf} / \mathrm{cm}^{2}$ e módulo de encruamento $K_{C}=-150000 \mathrm{kgf} / \mathrm{cm}^{2}$. Para as armaduras adotou-se $E_{S}=2700000$ $\mathrm{kgf} / \mathrm{cm}^{2}, \mathrm{~K}_{\mathrm{S}}=1400000 \mathrm{kgf} / \mathrm{cm}^{2}$, tensão de plastificação $\mathrm{F}_{\mathrm{y}}=2400 \mathrm{Kgf} / \mathrm{cm}^{2}$, área $\mathrm{A}_{\mathrm{s}}=0,5$ $\mathrm{cm}^{2} / \mathrm{cm}$ e posição $\mathrm{x}_{3}=12 \mathrm{~cm}$.

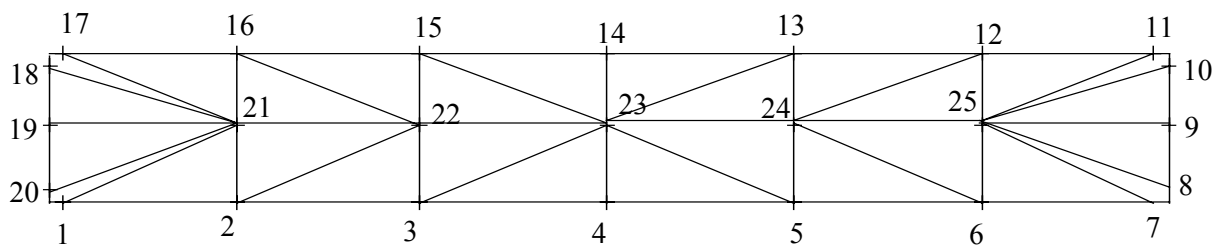

FIGURA 7.9 - Discretização da Viga

O contorno foi discretizado em 8 elementos, o que resulta em 20 pontos nodais no contorno e o domínio foi discretizado em 24 células, o que requer a definição de 5 pontos internos, como está mostrado na figura (7.9). O cálculo foi feito considerando-se dois pontos de colocação externos, sendo que suas posições foram definidas por $a_{1}=0,1$ e $a_{2}=0,25$, a tolerância adotada para a definição da convergência foi de $0,1 \%$. Para a integração das tensões considerou-se 8 pontos de Gauss, ao longo da espessura da placa. O modelo constitutivo utilizado para o concreto foi o elasto-plástico.

A carga foi aplicada em 30 incrementos, sendo que o primeiro foi definido para $\beta=0,57$. No segundo incremento, onde o coeficiente de multiplicação do carregamento $\beta$ era igual a 0,6, observou-se plastificação nas armaduras. $O$ concreto plastificou somente com $\beta=0,77$ e alcançou a deformação de 0,003 , que é a máxima permitida, $\operatorname{com} \beta=0,91$.

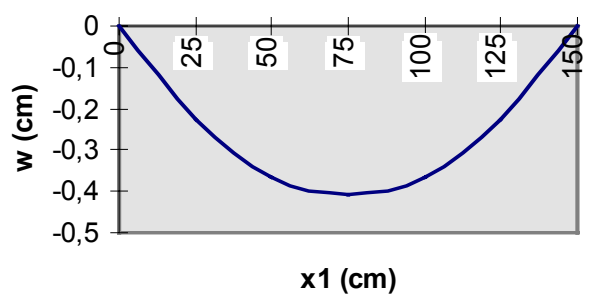

FIGURA 7.10 - Deslocamentos Transversais nos Pontos do Domínio 
A tabela (7.1) fornece os momentos na direção $\mathbf{x}_{1}$ e as flechas para $\beta=0,9$ e a figura (7.10) representa a elástica obtida para o mesmo incremento. A figura (7.11) mostra o deslocamento transversal do ponto central 23 (figura 7.9), ao longo do processo incremental

TABELA 7.1 - Resultados obtidos para $\beta=0,9$

\begin{tabular}{ccc}
\hline Node & $\mathbf{M}_{\mathbf{X} 1}(\mathrm{kgf.cm})$ & $\mathbf{w}(\mathrm{cm})$ \\
\hline 1 & 44983 & \\
\hline 2 & 44943 & \\
\hline 3 & 44969 & \\
\hline 4 & 44883 & \\
\hline 19 & 44995 & 0 \\
\hline 20 & 44995 & \\
\hline 21 & 44999 & 0,2272 \\
\hline 22 & 45038 & 0,3635 \\
\hline 23 & 45069 & 0,4090 \\
\hline 24 & & 0,3635 \\
\hline 25 & & 0,2272 \\
\hline
\end{tabular}

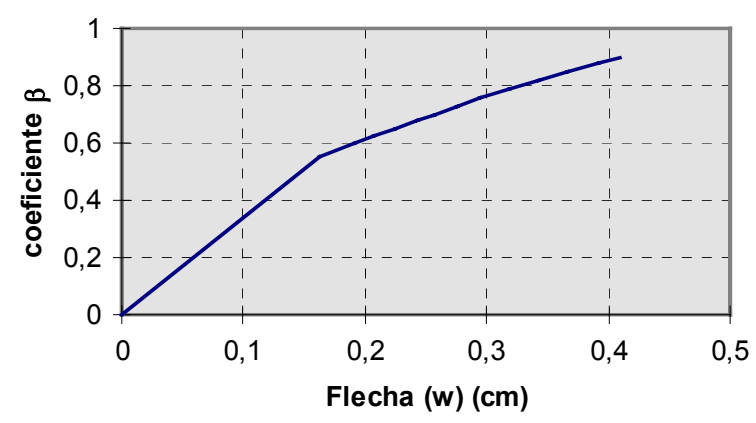

FIGURA 7.11 - Curva Carga-deslocamento do Ponto Central

Foi feito, ainda, um cálculo da viga considerando-se 16 elementos e 12 pontos de Gauss e os resultados obtidos foram muito próximos àqueles obtidos para 8 elementos e 8 pontos de Gauss, como está indicado na tabela (7.2).

A figura (7.12) mostra a influência do número de pontos de Gauss considerado ao longo da espessura da placa, para a integração das tensões, na curva carga-deslocamento do ponto central da viga. Observando-se a figura (7.12) e comparando-se com os resultados obtidos para $\mathrm{NG}=8$, pode-se concluir que a curva foi significamente alterada para $\mathrm{NG}=2$, 
onde o cálculo convergiu para $\beta=1$, notando-se, portanto, um aumento na carga limite e nos valores dos deslocamentos transversais. Para $\mathrm{NG}=6$, a carga limite permaneceu inalterada, porém o deslocamento nos últimos incrementos aumentou um pouco. Para $\mathrm{NG}=12$ e NG=4, observou-se uma diminuição nos valores da carga limite e do deslocamento.

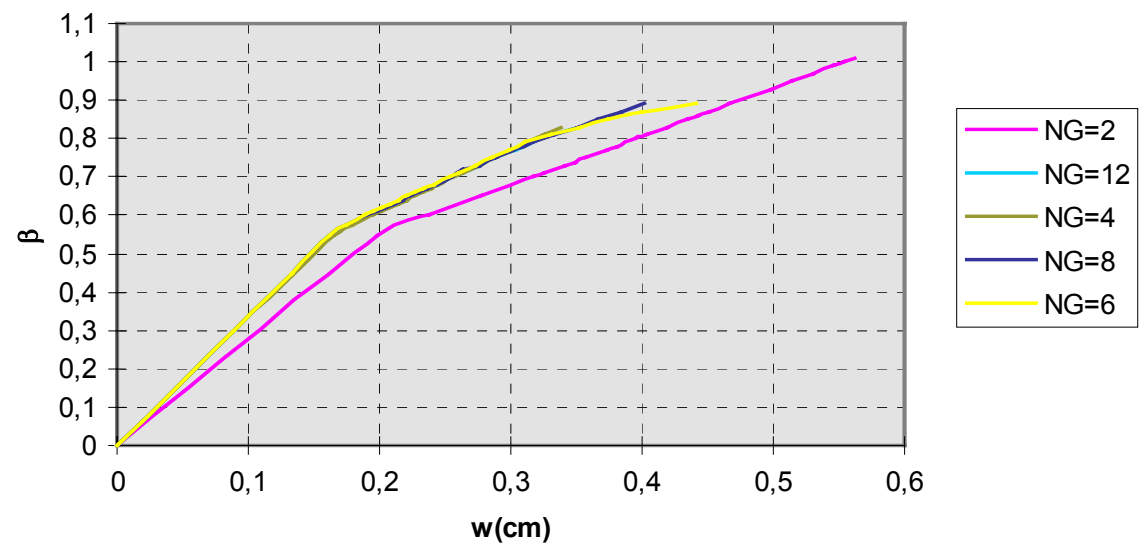

FIGURA 7.12 - Influência do Número de Pontos de Gauss (NG) na Curva Cargadeslocamento do Ponto Central da Viga

TABELA 7.2 - Comparação dos resultados obtidos com as duas malhas

\begin{tabular}{ccccccc}
\hline \multicolumn{7}{c}{ 8 elementos } \\
\hline & $\mathrm{NG}=2$ & $\mathrm{NG}=4$ & $\mathrm{NG}=6$ & $\mathrm{NG}=8$ & $\mathrm{NG}=12$ & $\mathrm{NG}=12$ \\
\hline $\mathrm{w}(\mathrm{cm})$ & 0,56137 & 0,33819 & 0,44142 & 0,40143 & 0,3512 & 0,41312 \\
\hline$\beta$ & 1 & 0,83 & 0,89 & 0,89 & 0,83 & 0,89 \\
\hline erro $(\%)$ de $\mathrm{w}$ & 35,9 & 18,1 & 6,85 & 2,83 & 14,99 & \\
\hline erro $(\%)$ de $\beta$ & - & 6,74 & 0 & 0 & 6,74 & \\
\hline
\end{tabular}

$\mathrm{Na}$ tabela (7.2), estão indicados o coeficiente $\beta$ correspondente à carga limite e o deslocamento do ponto central no último incremento, obtidos com as duas malhas. Tem-se, ainda, o erro relativo aos resultados obtidos com a malha de 16 elementos. Deve-se observar, que para $\mathrm{NG}=2$, a carga limite não foi encontrada.

\subsubsection{Placa apoiada com carga concentrada no centro}




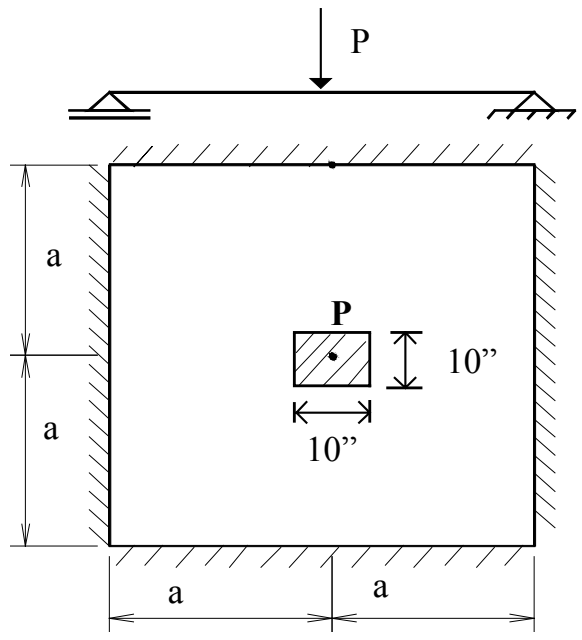

FIGURA 7.13 - Placa Apoiada com Carga Concentrada

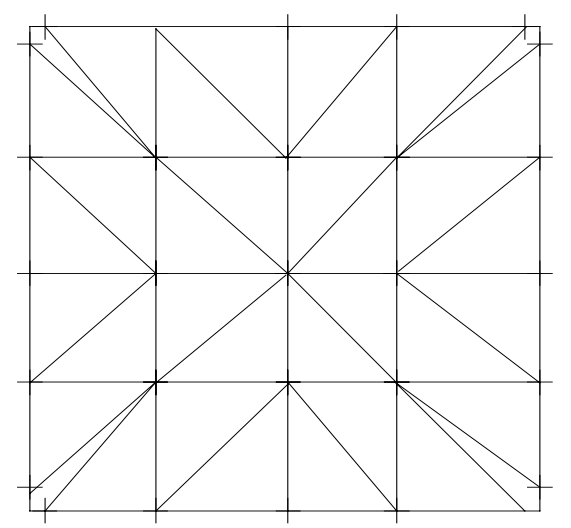

FIGURA 7.14 - Discretização da Placa

Nesse exemplo, serão considerados dois modelos constitutivos para o concreto: o elasto-plástico e o de dano. A placa indicada na figura (7.13) é apoiada nos quatro lados, tem dimensão $a=36$ " e espessura $t=5,5$ ". A carga aplicada no seu centro é distribuída sobre uma área de 10 "x10", resultando numa carga distribuída $\mathrm{g}=800 \mathrm{psi}$. No cálculo onde considerou-se o modelo elasto-plástico para o concreto, foram utilizadas duas malhas: numa primeira, o contorno da placa foi discretizado em 8 elementos e seu domínio em 32 células, o que requer 20 pontos nodais no contorno e 5 no domínio, como está indicado na figura (7.14) e outra onde o contorno foi discretizado em 16 elementos e o domínio em 128 células, resultando em 36 pontos nodais no contorno e 49 pontos internos. No caso do modelo de dano, foi considerado apenas a malha de 8 elementos e 32 células.

Têm-se armaduras idênticas nas direções $\mathbf{x}_{\mathbf{1}}$ e $\mathbf{x}_{2}$, as quais têm módulo de elasticidade $\mathrm{E}_{\mathrm{s}}=3000000 \mathrm{psi}$, módulo de encruamento $\mathrm{K}_{\mathrm{S}}$ nulo, área transversal $\mathrm{A}_{\mathrm{s}}=0,04455^{\prime 2} /$ ", 
tensão de plastificação $\mathrm{F}_{\mathrm{y}}=44000$ psi e posição $\mathrm{x}_{3}=1,75$ ". $\mathrm{O}$ concreto tem módulo de elasticidade $\mathrm{E}_{\mathrm{C}}=4000000$ psi, tensão de plastificação $\mathrm{f}_{\mathrm{C}}=6920 \mathrm{psi}$, coeficiente de poisson $\mathrm{v}$ $=0,15$ e módulo de encruamento negativo $\mathrm{K}_{\mathrm{C}}=-5448819$ psi. A análise foi feita considerando-se dois pontos de colocação externos, sendo que as posições dos mesmos foram definidas por $a_{1}=0,1$ e $a_{2}=0,25$; a tolerância adotada para o critério de convergência foi de $0,1 \%$. Foram utilizados 8 pontos de Gauss ao longo da espessura.

Este exemplo foi analisado também por CORRÊA (1991), através do método dos elementos finitos, onde um oitavo da laje foi discretizado em 16 elementos e por CHUEIRI (1994), onde o mesmo discretiza um quarto do contorno da laje em 8 elementos e o domínio em 64 células.

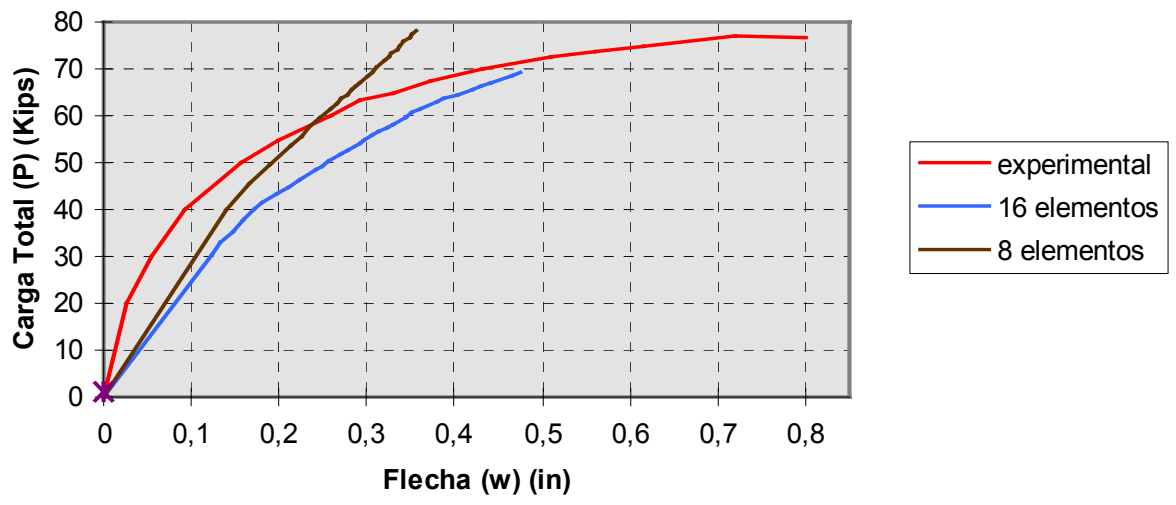

FIGURA 7.15 - Curva Carga-Deslocamento no Ponto Central

$\mathrm{Na}$ figura (7.15) está indicada a curva carga-deslocamento do ponto central, obtida considerando-se o modelo elasto-plástico para o concreto, para as duas malhas consideradas. No caso da malha de 8 elementos, a carga total de 80 Kips foi aplicada em 28 incrementos, sendo que a plastificação na armadura do ponto central foi observada no segundo incremento, para a carga de 45,6Kips. Para a carga de 77Kips, que corresponde à carga limite observada experimentalmente, não observou-se nenhuma plastificação no concreto e o cálculo convergiu. No caso da malha de 16 elementos, o cálculo foi feito considerando-se 37 incrementos, sendo que nos últimos 25 , o coeficiente $\beta$ foi igual a 0,01 . A plastificação ocorreu para $\beta=0,44$, o que corresponde a $\mathrm{P}=35,2$ Kips. A armadura atingiu a deformação limite de 0,02 , no ponto central, para a carga $\mathrm{P}=69,4 \mathrm{Kips}$, onde o concreto não estava plastificado. Pode-se observar que a diferença entre as duas respostas é muito grande. 
A figura (7.16) representa a curva momento-curvatura do ponto central, considerando-se a malha de 8 elementos. Ainda com a malha de 8 elementos, estudou-se a influência do número de pontos de Gauss considerado ao longo da espessura na curva cargadeslocamento do ponto central e constatou-se que as respostas foram praticamente idênticas para NG igual a 4, 6, 8 ou 12. Para $\mathrm{NG}=2$, observou-se dificuldades para zerar a normal.

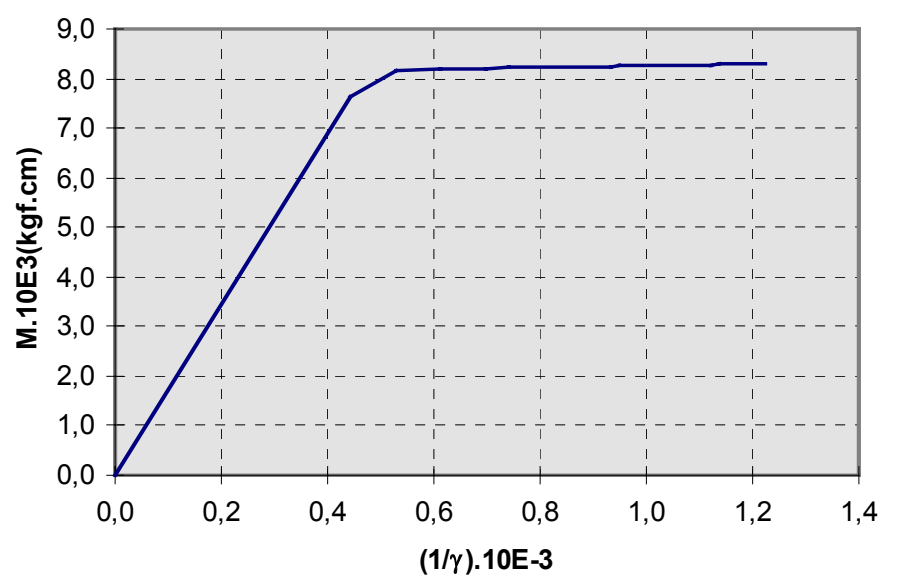

FIGURA 7.16 - Curva Momento-Curvatura na Direção $\mathrm{x}_{1}$ no ponto Central
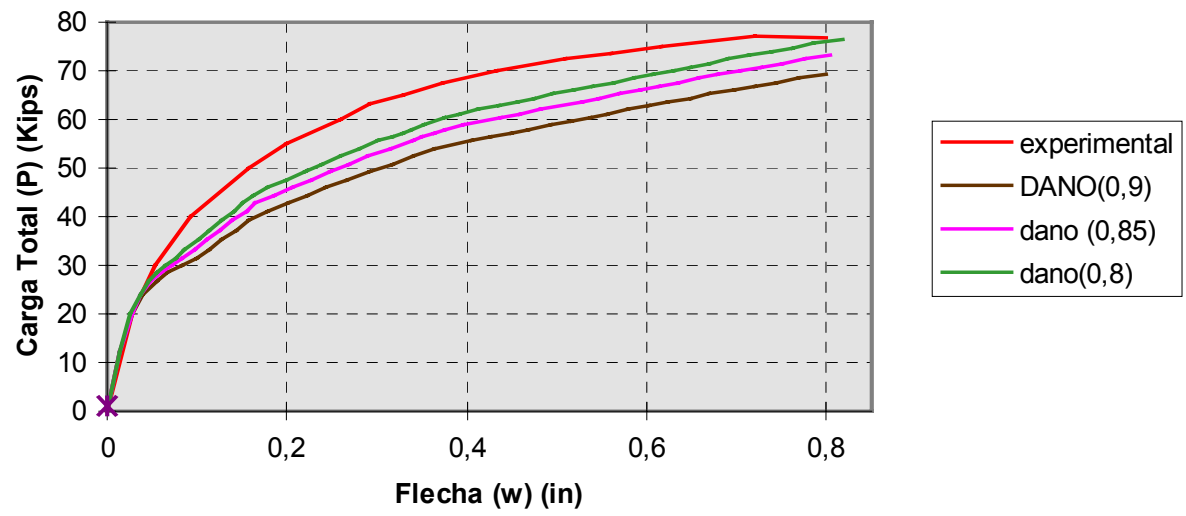

FIGURA 7.17 - Curva Carga-Deslocamento no Ponto Central, Considerando o Modelo de

Dano

A figura (7.17) mostra algumas curvas carga-deslocamento do ponto central obtidas considerando-se o modelo de dano para o concreto, onde limitou-se o valor da variável de dano $\mathbf{D}$, dada por (6.84), em 0,$9 ; 0,85$ e 0,8 . O cálculo foi feito considerando-se 48 incrementos, sendo que nos últimos 27 incrementos o coeficiente $\beta$ era igual a 0,01 . No caso 
onde limitou-se o dano em 0,8 , observou-se plastificação na armadura do ponto central para $\mathrm{P}=46$ Kips e o concreto atingiu a deformação máxima permitida de 0,003 , no ponto central, para $\mathrm{P}=76,4$ Kips.

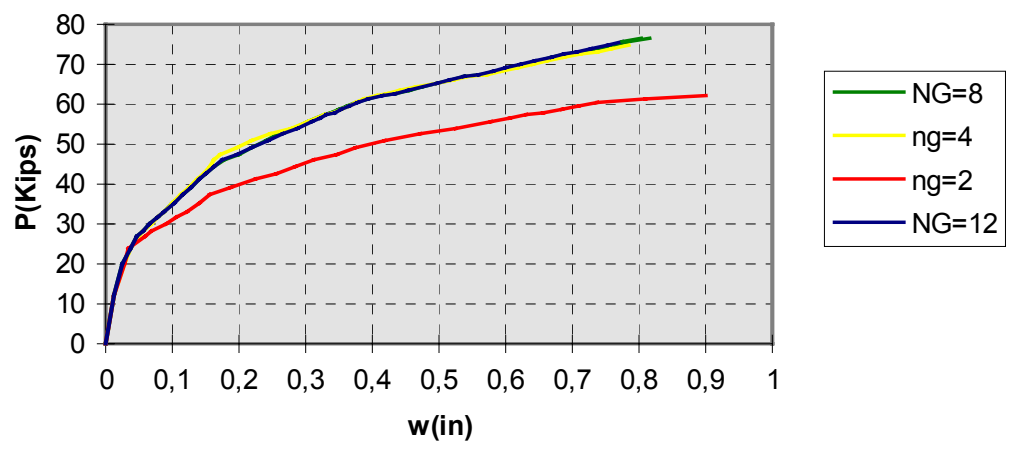

FIGURA 7.18 - Influência do Número de Pontos de Gauss na Curva Carga-Deslocamento do Ponto Central, Considerando Modelo de Dano

A influência do número de pontos de Gauss ao longo da espessura na curva carga deslocamento do ponto central está indicada na figura (7.18). As cargas limites e os deslocamentos no último incremento obtidos para $\mathrm{NG}=2, \mathrm{NG}=4, \mathrm{NG}=8$ e $\mathrm{NG}=12$ estão indicados na tabela (7.3), assim como os erros relativos aos resultados obtidos experimentalmente.

TABELA 7.3 - Influência do número de Gauss no deslocamento e na carga limite

\begin{tabular}{cccccc}
\hline & \multicolumn{3}{c}{8 elementos } & experimental \\
\hline & $\mathrm{NG}=2$ & $\mathrm{NG}=4$ & $\mathrm{NG}=8$ & $\mathrm{NG}=12$ \\
\hline $\mathrm{w}$ (in) & 0,90 & 0,785 & 0,817 & 0,80 & 0,8 \\
\hline $\mathrm{P}($ Kips) & 62,0 & 74,80 & 76,40 & 76,4 & 77 \\
\hline erro (\%) de $\mathrm{w}$ & 12,5 & 1,875 & 2,125 & 0 & \\
\hline erro (\%) de $\beta$ & 19,4 & 2,860 & 0,80 & 0,80 & \\
\hline
\end{tabular}

7.6.3 Placa apoiada nos cantos com carga concentrada no centro

A placa indicada na figura (7.19) foi analisada considerando-se o modelo de dano e o elasto-plástico para o concreto. No caso do modelo de dano, o cálculo feito com 8 elementos e 32 células, somente convergiu até o incremento imediatamente anterior à plastificação da 
armadura, o que corresponde, aproximadamente, à metade da carga total. Na análise elastoplástica, foram consideradas as mesmas malhas de 8 e 16 elementos do exemplo anterior.

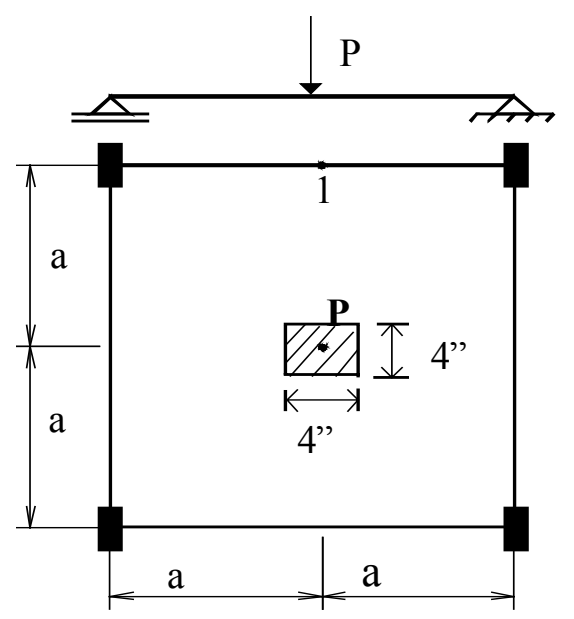

FIGURA 7.19 - Placa apoiada nos cantos com carga concentrada

As dimensões da placa são as seguintes: $a=18$ " e a espessura $t=1,75$ " e para $o$ concreto, considerou-se: $\mathrm{E}_{\mathrm{C}}=4,15 \mathrm{E} 6 \mathrm{psi}, \mathrm{f}_{\mathrm{C}}=5500$ psi, $\mathrm{v}=0,15$ e módulo de encruamento $\mathbf{K}_{\mathbf{C}}$ nulo. Têm-se armaduras idênticas nas direções $\mathrm{x}_{1}$ e $\mathrm{x}_{2}$, para as quais adotou-se: $\mathrm{E}_{\mathrm{S}}=$ 29E6 psi, $\mathrm{F}_{\mathrm{y}}=29000 \mathrm{psi}, \mathrm{A}_{\mathrm{s}}=0,011135 " 2 / ", \mathrm{x}_{3}=0,435 "$ e variou-se o módulo de encruamento $\mathrm{K}_{\mathrm{S}}$, como está indicado na figura (7.20). A carga total de 3,2 Kips foi distribuída numa área de 4"x4", como indica a figura (7.19), resultando em uma carga distribuída de 200 psi. Considerou-se 8 pontos de Gauss ao longo da espessura, uma tolerância de convergência de $0,1 \%$ e pontos de colocação externos, sendo que suas posições foram definidas por $\mathrm{a}_{1}=0,2$ e $\mathrm{a}_{2}=0,5$.

$\mathrm{Na}$ figura (7.20) estão representados os resultados obtidos com as duas malhas, sendo que o módulo de encruamento do aço utilizado foi igual a $20 \%$ do seu módulo de elasticidade para a malha de 8 elementos e $8 \%$ de $\mathbf{E}_{\mathbf{s}}$ para a malha de 16 elementos. Observando-se a figura (7.20), conclui-se que a diferença entre os resultados obtidos com as duas malhas são bastante significativas. O cálculo, considerando-se a malha de 16 elementos, foi dividido em 51 incrementos, adotando-se $\beta=0,43$ para o primeiro incremento e $\beta=0,01$ para os outros. A plastificação no aço ocorreu para $\mathrm{P}=1,41$ kips e a carga limite foi de 2,98Kips, quando a armadura do ponto central atingiu a deformação de 0,02. Foi feito também um cálculo considerando-se a malha de 16 elementos e $\mathrm{K}_{\mathrm{S}}=0$. Nesse caso, a carga limite foi alcançada logo após a plastificação. 


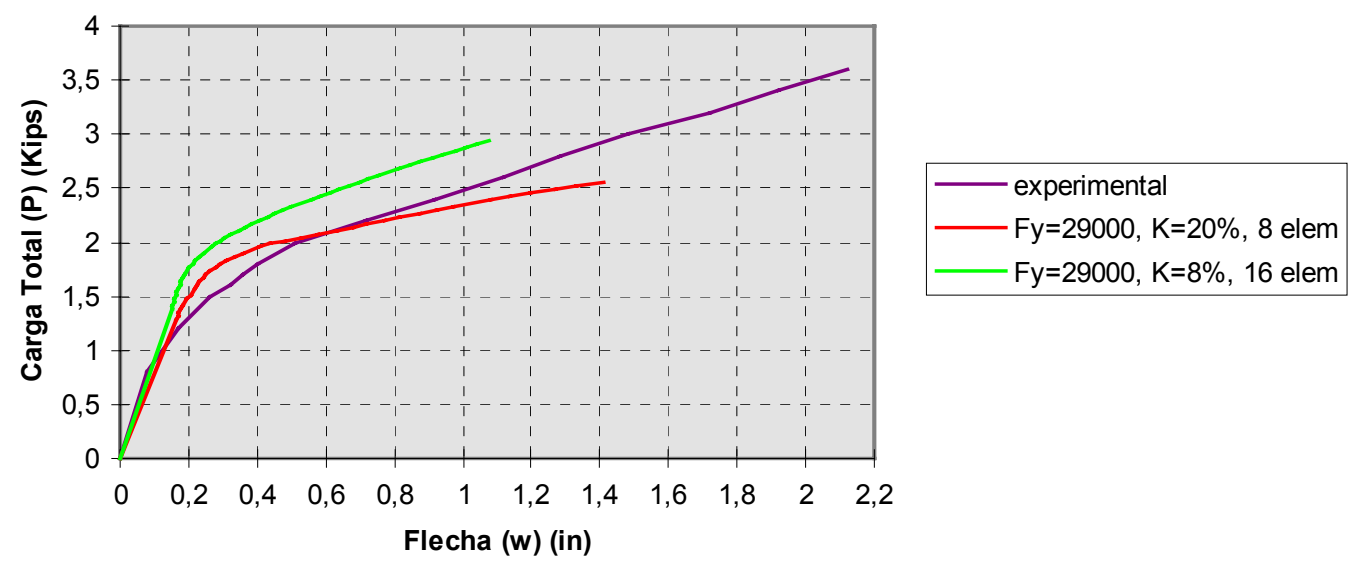

FIGURA 7.20 - Curvas Carga-Deslocamento para o Ponto 1, Considerando-se Duas Malhas Diferentes e Variando $\mathrm{K}_{\mathrm{s}}$

Esse exemplo foi analisado também por FIGUEIRAS (1983), CORRÊA (1991) e CHUEIRI (1994). Nos dados fornecidos experimentalmente, não havia referência à resistência $F_{y}$ do aço. Assim, FIGUEIRAS (1983) adotou $F_{y}=6000$ psi e CHUEIRI (1994) adotou $\mathrm{F}_{\mathrm{y}}=5000$ psi. 


\section{CONCLUSÕES}

A formulação do MEC para a análise de placas, utilizando-se a teoria clássica de Kirchhoff, mostrou-se ser eficiente para a solução elástica linear de placas, sob diversas condições de contorno e carregamento.

O emprego da técnica de sub-elementos, aumentou significamente a precisão do cálculo, possibilitando a obtenção de bons resultados, mesmo considerando-se uma discretização pobre do contorno.

Para a escolha da posição dos pontos de colocação, foram considerados três esquemas: um que considera um ponto no contorno e outro fora, outro onde se considera dois pontos de colocação fora do domínio e, ainda, um terceiro, onde adota-se os dois pontos no contorno. Obteve-se bons resultados, para o primeiro e o segundo esquemas, porém, observou-se que o sistema de equações obtido com o terceiro era singular. Tal singularidade poderia ser removida, ao considerar um ponto de colocação fora do contorno.

A viabilidade da utilização do Método dos Elementos de Contorno para a análise de placas foi comprovada pela precisão dos resultados obtidos. O método possibilita modelar placas com geometria qualquer, inclusive com descontinuidades de vinculação no contorno, discretizando-se apenas o contorno, o que representa uma vantagem em relação a outros métodos numéricos, onde há a necessidade também da discretização do domínio. Deve-se ressaltar, que a área onde está distribuído o carregamento, pode ter forma qualquer e é independente da discretização do contorno.

A inclusão de campos de momentos iniciais na formulação obtida considerando-se carregamentos transversais, possibilita a análise de placas sujeitas a efeitos de temperatura e 
retração, bem como a análise não-linear. $\mathrm{Na}$ análise não-linear utilizou-se um algoritmo incremental-iterativo, baseado no método das tensões iniciais e no Método de NewtonRaphson Modificado, onde as matrizes envolvidas não são atualizadas a cada iteração. Entretanto, a necessidade de um maior número de iterações para se alcançar a convergência do cálculo, não implica em maiores prejuízos no tempo de processamento, uma vez que as operações matriciais realizadas a cada iteração são bastante simples.

O cálculo dos esforços ao longo da espessura da placa foi feito considerando-se um modelo estratificado, onde a placa é dividida em camadas nas quais verificam-se os modelos constitutivos adotados para as armaduras e o concreto. O tensor de momentos internos é obtido, integrando-se numericamente as tensões na placa de concreto, de tal forma que a normais resultantes nas direções $\mathbf{x}_{1}, \mathbf{x}_{2}$ e $\mathbf{x}_{1} \mathbf{x}_{2}$ sejam nulas. Esse modelo mostrou-se ser eficiente e conveniente, pois permite a introdução de critérios diferentes em cada camada. Utilizaram-se dois tipos de modelos constitutivos para o concreto: um elasto-plástico e um de dano. Ambos os modelos, apesar de serem simples, mostraram-se ser estáveis, pois eram capazes de encontrar a carga limite e convergiam para a solução exata com o refinamento da malha ou o aumento do número de pontos de Gauss considerado na integração numérica das tensões. Constatou-se que os resultados obtidos para 8 ponto de Gauss eram bastante razoáveis.

Esse trabalho é a base para uma análise mais refinada de placas de concreto armado. Assim, considerando-se modelos constitutivos mais complexos, pode-se obter modelos que representem melhor o comportamento do concreto e utilizando-se o Método de NewtonRaphson padrão, onde as matrizes envolvidas são atualizadas a cada iteração, pode-se aumentar a velocidade de convergência. Além disso, transformando-se as integrais de domínio sobre as células, em integrais sobre o contorno das mesmas, pode-se implementar a técnica de sub-elementos na integração numérica das células, aumentando, assim, a precisão do cálculo. 


\section{REFERÊNCIAS BIBLIOGRÁFICAS}

ALTIERO, N.J.; SIKARSKIE, L. D. (1978). A boundary integral method applied to plates of arbitrary plan form. Comp. \& Struct., n.9, p.163-168.

ÁLVARES, M.S. (1993). Estudo de um modelo de dano para o concreto: formulação, identificação paramétrica e aplicação com emprego do método dos elementos finitos. São Carlos. 123p. Dissertação (Mestrado) - Escola de Engenharia de São Carlos, Universidade de São Paulo.

AUFARE, M.; CHAUVEL, D.; L'HUBY, Y. (1986). Dynamic elasto-plastic analysis of reinforced concrete slabs and application to plastic design of some building structures. In: HINTON, E.; OWEN, R., eds. Computational modelling of reinforced concrete structures. Swansea: Pineridge Press. p.303-326.

BARRET, K.E.; ELLIS, S. (1988). An exact theory of elastic plates. Int. J. Solids Struct., v.24, n.9, p.859-880.

BETTI, E. (1872). Teoria dell elasticita. Il Nuovo Ciemento, p.7-10.

BÉZINE, G.P. (1978). Boundary integral formulation for plate flexure with arbitrary boundary conditions. Mech. Res. Comm., v.5, n.4, p.197-206.

BÉZINE, G.P.; GAMBY, D.A. (1978). A new integral equation formulation for plate bending problems. In: BREBBIA, C.A., ed. Recent advances in BEM. London: Pentech Press, p.327342.

BÉZINE, G.P. (1980). A mixed boundary integral: finite element approach to plate vibration problems. Mech. Res, Comm., v.7, n.3, p.141-150.

(1981). A boundary integral equation method for plate flexure with conditions inside the domain. Int. J. Num. Meth. Engrg, n.17, p.1647-1657.

BÉZINE, G.A.; CIMETIERRE, A.; GELBERT, J.P. (1985). Unilateral buckling on thin plates by the boundary integral equation method Int. J. Num. Meth. Engrg, n.21, p.2189-2199.

BREBBIA, C.A. (1978). The boundary element method for engineers. London: Pentech Press.

BREBBIA, C.A.; TELLES, J.C.F.; WROBEL, L.C. (1984). Boundary element techniques theory and applications in engineering. Berlin: Springer-Verlag.

BUI, H.D. (1978). Some remarks about the formulation of three-dimensional thermoelastic problems by integral equations. Int. J. Solids Structures, v.14, p.935-939.

BUSSAMRA, F. L. S. (1993). Equações constitutivas do concreto baseadas na mecânica do dano contínuo. Dissertação (Mestrado) - Escola de Engenharia de São Carlos, Universidade de São Paulo. 
CALDERÓN E. T. (1991). Uma formulação alternativa para o cálculo de placas sobre fundação elástica pelo método dos elementos de contorno. São Carlos. 191p. Dissertação (Mestrado) - Escola de Engenharia de São Carlos, Universidade de São Paulo.

. (1996). Sobre o uso do método dos elementos de contorno - MEC para o estudo de interação de placas com o meio contínuo. São Carlos. 247p. Tese (Doutorado) - Escola de Engenharia de São Carlos, Universidade de São Paulo.

CHANNAKESKAVA, C. et al. (1988). Elasto-plastic-cracking analysis of reinforced concrete structures using boundary element method. In: BREBBIA, C. A., ed. Boundary elements $X, \mathrm{v}$. 3. Computational Mechanics Publications.

CHAVES, E.W.V. (1997). Análise de placas com variação de espessura através do método dos elementos de contorno. São Carlos. 171p. Dissertação (Mestrado) - Escola de Engenharia de São Carlos, Universidade de São Paulo.

CHENG, S. (1979). Elastic theory of plates and a refined theory. J. Appl. Mech., n.46, p.644650.

CHUEIRI, L.H.M. (1994). Formulação do método dos elementos de contorno para análise elastoplástica de placas. São Carlos. 219p. Tese (Doutorado) - Escola de Engenharia de São Carlos, Universidade de São Paulo.

CORRÊA, M.R.S. (1991). Aperfeiçoamento de modelos usualmente empregados no projeto de sistemas estruturais de edifícios. São Carlos. 331p. Tese (Doutorado) - Escola de Engenharia de São Carlos, Universidade de São Paulo.

COSTA Jr.; BREBBIA C.A. (1985). Elastic buckling of plates using the boundary element method. In: BREBBIA, C.A.; MAIER, G. eds. Boundary element VII. Berlin, SpringerVerlag.

DANSON, D.J. (1979). Analysis of plate bending problems by direct boundary element method. Southampton. Dissertation (M.Sc.) - University of Southampton.

DRIEMEIER, L. (1995). Considerações sobre a fadiga em metais e o comportamento do concreto sob solicitação cíclica. São Carlos. 120p. Dissertação (Mestrado) - Escola de Engenharia de São Carlos, Universidade de São Paulo.

FIGUEIRAS, J.A. (1983). Ultimate load analysis of anisotropic and reinforced concrete plates and shells. Swansea. Tese (Doutorado) - University College of Swansea.

FREDHOLM, I. (1903). Sur une Classe d'equations fonctionelles. Acta Math., 27, p.365-390.

GIL RODRIGUEZ, J.C. (1986). Sobre o emprego do método dos elementos de contorno em problemas elásticos bidimensionais. São Carlos. Dissertação (Mestrado) - Escola de Engenharia de São Carlos, Universidade de São Paulo. 
HAN, R.P.S.; MOU, Y. (1993). Void induced damage in ductile materials. In: $14^{\text {th }}$ CANCAM, Kingston, Ontario.

HAND, F.R.; PECKNOLD, D.A.; SCHNOBRICH, W.C. (1973). Nonlinear layered analysis of RC plates and shells. J. Struct. Div., ASCE, v.99, n.ST7, p.1491-1505, July.

HANSEN, E.B. (1976). Numerical Solution of integro-differential and singular integral equations for plate bending problems. Journal of Elasticity v.6, n.1, p.39-56.

HARTMANN, F.; ZOTEMANTEL, R. (1986). The direct boundary element method in plate bending. Int. J. Num. Meth. Engrg., v.23, n.11, p.2049-2069.

HENCHY, H. (1947). Uber die berucksichtigung der schubverzerrungen in ebenen platten. Ing. Arch., n.16.

HILL, R. (1950). The mathematical theory of plasticity. Oxford: Oxford University Press.

HU, H.; SCHNOBRICH, W.C. (1991). Nonlinear finite element analysis of reinforced concrete plates and shells under monotonic loading. Comput. \& Struct., n.38, p.637-651.

JASWON M.A.; MAITI, M.; SYMM, G.J. (1967). Numerical biharmonic analysis and some applications. Int. J. Solids Structures, n.3, p.309-332.

JOFRIET, J.C.; McNEICE, G.M. (1971). Finite element analysis of reinforced concrete slabs. $J$. Struct. Div., ASCE, n.97, p.785-806, Mar.

KACHANOV, L.M. (1958). Time of the rupture process of non-linear solid mechanics. Otd. Tech. Nauk., v.8,p.28-31.

(1984). On brittle fracture of a thin plastic interlayer in creep conditions. In: DVORAK, G.; SHIELD, R., ed. Mechanics of material behavior. Amsterdam, Elsevier.

KAMYIA, N. et al. (1982). An approximate finite deflection analysis of a heated elastic plate by the boundary element method. Appl. Math. Modelling, v.6, n.1, p.23-27.

KARAM, V.J.; TELLES, J.C.F. (1992). BEM applied to plate bending elastoplastic analysis using Reissner's theory. Engineering Analysis with Boundary Elements, v.9, n.4, p.351-357.

KATSIKADELIS, J.T.; ARMENAKAS, A.E. (1984). Analysis of clamped plates on elastic fondation by the boundary integral method. J. Appl. Mech., ASCE, v.110, n.7, p.1085-1104.

KIRCHHOFF, G. (1850). Uber das gleichgewicht und die bewegung einer elastischen scleibe. $J$. Math., n.40, p.51-58.

KROMM, A. (1953). Verallgemeinerte theorie der plattenstatik. Ing. Arch., n.21.

KUPRADZE, V.D. (1965). Potential methods in the theory of elasticity. Jerusalem: Israel Program for Scientific Translations. 
LACHAT, J.C. (1975). A further development of boundary integral techniques for elastostatics. Southampton. Thesis (Ph.D.) - University of Southampton.

LEMAITRE, J. (1984). How to use damage mechanics. Nucl. Eng. Des., v.80, p.233.

LEMAITRE, J.; CHABOCHE, J.L. (1974). A non-linear model of creep-fatigue damage cumulation and interation. In: IUTAM SYMP. OF MECHANICS OF VISCOELASTIC MEDIA AND BODIES, Gothenburg,1974. Proc. Springer.

(1985). Mécanique des matériaux solids. Paris, Dunod-Bordas.

LEVINSON, M. (1980). An accurate simple theory of statics and dynamics of elastic plates. Mech. Res. Comm., v.7, n.6, p.343-350.

LIN, C.S.; SCORDELIS, A.C. (1975). Nonlinear analysis of RC shells of general form. J. Struct. Div., ASCE., n.101, p.523-538, mar.

MANZOLI, O.L. (1992). Formulação do método dos elementos de contorno para placas sobre fundação elástica. São Carlos. Dissertação (Mestrado) - Escola de Engenharia de São Carlos, Universidade de São Paulo.

MARIGO, J.J. (1985) Modeling of brittle and fatigue damage for elastic material by growth of microvoid. Eng. Fract. Mech.,v.21,p.861.

MAZARS, J. (1984). Application de la mécanique de l'endommagement au comportement non linéaire et à la rupture du béton de structure. Paris, 1984. Thèse (Doctorat d'etat) - université Paris 6.

MENDELSON, A. (1968). Plasticity: theory and application. [S. 1.]: Robert E. Krieger Publ. Co..

MIKHLIN, S.G. (1962). Singular integral equation. American Math. Soc. Trans. Series 1, n.10, p.84-197.

MINDLIN, R.D. (1951). Influence of rotatory inertia and shear on flexural motions of isotropic, elastic plates. J. Appl. Mech., n.13, p.31-38.

MORJARIA, M.; MUKHERJEE, S. (1980). Inelastic analysis of transverse deflection of plates by the boundary element method. J. Appl. Mech., ASME, v.47, n.2, p.291-296.

MOSHAIOV, A.; VORUS, W.S. (1986). Elasto-plastic plate bending analysis by a boundary element method with initial plastic moments. Int. J. Solids Strucutres, v.22, n.11, p.12131229.

MURAKAMI, S. (1981). Effects of cavity distribution in constitutive equations of creep and creep damage. In: EUROMECH COLLOQUE ON DAMAGE MECHANICS, Cahan, France, 1981. 
OLIVEIRA NETO, L. (1991). Análise de placas de borda curva pelo método dos elementos de contorno. São Carlos. 118p. Dissertação (Mestrado) - Escola de Engenharia de São Carlos, Universidade de São Paulo.

OWEN, D.R.J.; HINTON, E. (1980). Finite elements in plasticity: theory and practice. Swansea, U. K.: Pineridge Press Limited.

PAIVA, J.B. (1987). Formulação do método dos elementos de contorno para flexão de placas e suas aplicações em engenharia de estrutura. São Carlos. 195p. Tese (Doutorado) - Escola de Engenharia de São Carlos, Universidade de São Paulo.

PROENÇA, S.P.B. (1988). Sobre modelos matemáticos do comportamento do concreto: análise crítica e contribuições. São Carlos. 330p. Tese (Doutorado) - Escola de Engenharia de São Carlos, Universidade de São Paulo.

REISSNER, E. (1944). On bending of elastic plates, J. Math. Physics, n.23, p.184-191.

. (1945). The effect of transverse shear deformation on bending of elastic plates. $J$. Appl. Mech, n.12.

(1986). On small deflections of shear deformable elastic plates. Meth. Appl. Mech. Engrg., n.59, p.227-233.

(1991). On the asymptotic expansions for the sixth-order linear theory problem of transverse bending of orthotropic elastic plates. Comput. Meth. Appl. Mech. Engrg., v.85, n.1, p.75-88.

RIBEIRO, G.O. (1992). Sobre a formulação do método dos elementos de contorno de placas usando as hipóteses de Reissner. São Carlos. 266p. Tese (Doutorado) - Escola de Engenharia de São Carlos. Universidade de São Paulo.

RIZZO, F. (1967). An integral approach to boundary value problems of classical elastostatics. Quaterly of Applied Mathematics, v.25, n.1, p.83-92.

SILVA, N.A. (1988). Aplicação do método dos elementos de contorno à análise de placas sobre fundações elásticas. São Carlos. 162p. Dissertação (Mestrado) - Escola de Engenharia de São Carlos, Universidade de São Paulo.

(1996). Aplicação do método dos elementos de contorno às placas com enrijecedores utilizando a teoria de Reissner. São Carlos. 230p. Tese (Doutorado) - Escola de Engenharia de São Carlos, Universidade de São Paulo.

SIMO, J.C.; JU, J.W. (1987). Stress and strain based continuum damage models: I. Formulation. Int, J. Solids Structures, v.23, p.821.

STERN, M. (1979). A general boundary integral formulation for the numerical solutions of plate bending problems. Int. J. Solids Structures, n.15, p.769-782. 
. (1983). Boundary integral equations for bending of thin plates. In: BREBBIA, C.A. ed. Progress in boundary element methods, vol.2. London, Pentech Press.

TAI, W.H. (1990). Plastic damage and ductile fracture in mild steels. Eng, Fract. Mech., v.37, p.853.

TELLES, J.C.F.; BREBBIA, C.A. (1979). On the application of the boundary element method to plasticity. Appl. Math. Modelling, n.3, p.466-470.

TIMOSHENKO, S.; WOINOWSKY-KRIEGER, S. (1959). Theory of plates and shells. New York: McGraw-Hill.

TOTTENHAN, H. (1979). The boundary element method for plates and shells. In: BANERJEE, P. K.; BUTTERFIELD, R. eds. Developments boundary element methods 1. London: Applied Science Publ., p.173-205.

VAN DER WEEËN, F.V. (1982). Application of the direct boundary element method to Reissner's plate model. In: BREBBIA, C.A. ed. Boundary element methods in engineering. Berlin, Springer-Verlag.

VENTURINI, W.S. (1982). Application of the boundary element formulation to solve geomechanical problems. Southampton. Thesis (Ph.D.) - University of Southampton.

YANG, W.H. (1980) A generalized Von Mises criterion for yield and fracture. J. Appl. Mech., $A S M E$, v.47,p.297-300.

WU, B.C.; ALTIERO, N.J. (1979). A boundary integral method applied to plates of arbitrary plan form and arbitrary boundary conditions. Comput. \& Struct., n.10, p.703-707.

ZIENKIEWICZ, O.C.; VALLIAPAN, S.; KING, I.P. (1969). Elasto-plastic solutions of engineering problems - initial stress, finite element approach. Int. J. Num. Meth. Engrg., n.1, p.5-100. 